
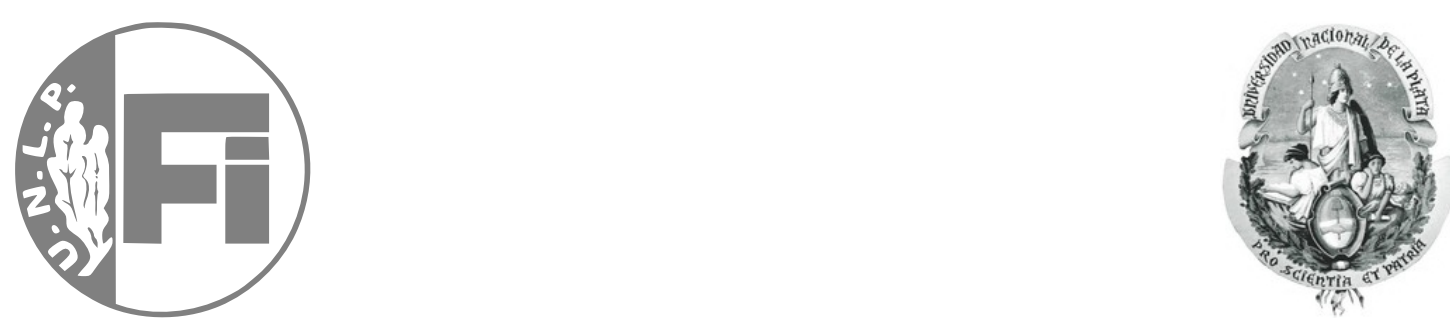

\title{
Influencia de segundas fases en las propiedades electromecánicas de materiales ferroeléctricos
}

\author{
Tesis de doctorado
}

\section{Cristian José Bottero}

\author{
Presentada ante la Facultad de Ingeniería \\ de la Universidad Nacional de La Plata
} como requisito para la obtención del grado académico de

\section{DOCTOR EN INGENIERÍA}

Dirección de tesis: Dr. Ing. Martín Ignacio Idiart

Jurado de tesis: Dr. Ing. Guillermo Etse

Dr. Ing. Sebastián Giusti

Dr. Lic. Marcelo Stachiotti

Fecha de la defensa oral y pública: 20 de marzo de 2018 

A mi familia, por el apoyo y por estar siempre presentes и моей дорогой Лене, за ее любовь, терпение и безусловную поддержку. 



\section{Resumen}

Este trabajo se centra en el estudio de materiales ferroeléctricos, es decir, en materiales que poseen la capacidad de mantener una polarización eléctrica permanente que puede ser alterada mediante la aplicación de un campo eléctrico externo. Debido a que el cambio en la polarización suele estar acompañado de una deformación mecánica, estos materiales son electrodeformables, y como tales, pueden utilizarse como sensores, actuadores, y otros microdispositivos.

La búsqueda de materiales electrodeformables con propiedades específicas no disponibles en materiales ferroeléctricos monolíticos ha motivado el desarrollo de una creciente variedad de materiales compuestos ferroeléctricos bifásicos con microestructuras de tipo matriz-inclusión. Debido a las características de los procesos de fabricación y la heterogeneidad microestructural de estos materiales, sus propiedades electromecánicas finales, incluso las piezoeléctricas, dependen de la respuesta electromecánica no lineal y disipativa del material ferroeléctrico durante el proceso de polarización. La respuesta del material compuesto frente a ciertos estados de carga es altamente disipativa, lo que tiene su origen en la fuerte histéresis eléctrica de la fase ferroeléctrica, que a su vez depende de la frecuencia del campo eléctrico aplicado. Más aún, cuando el estado de cargas involucra solicitaciones tanto eléctricas como mecánicas, el comportamiento del compuesto depende también de la histéresis mecánica de los materiales constitutivos. Caracterizar experimentalmente respuestas electromecánicas hereditarias bajo historias de carga generales en función de la microestructura resulta pues impracticable y surge así la necesidad de desarrollar modelos micromecánicos capaces de predecir con aceptable precisión la influencia de segundas fases en la respuesta electromecánica de materiales ferroeléctricos.

En este trabajo se establecen en primer lugar relaciones entre el comportamiento macroscópico y el comportamiento de las fases constitutivas en materiales compuestos ferroeléctricos con microestructuras complejas y sometidos a historias de carga arbitrarias. El comportamiento ferroeléctrico de cada fase se describe mediante una densidad de energía almacenada y un potencial de disipación en el marco de los 'materiales estándar generalizados'. Discretizando en el tiempo las ecuaciones diferenciales correspondientes siguiendo un esquema implícito, se obtiene una representación variacional de la respuesta macroscópica del compuesto que involucra un solo potencial incremental. Las estimaciones teóricas se obtienen luego mediante ciertas 'microgeometrías resolubles' cuyo potencial incremental macroscópico puede determinarse en forma exacta. Por ser exactas para una clase de materiales, es- 
tas estimaciones satisfacen automáticamente todos las características comúnmente deseadas en una aproximación teórica: estar de acuerdo con restricciones materiales, satisfacer todas las cotas pertinentes, y poseer todas las propiedades de convexidad correspondientes. La metodología propuesta se aplica al estudio de la influencia de inclusiones de segundas fases en una matriz ferroeléctrica de titanato zirconato de plomo, con inclusiones metálicas, ferromagnéticas y poros. En particular, se evalúa el rol de las fluctuaciones en la polarización remanente en las propiedades piezoeléctricas de los compuestos y se comparan estos resultados con una técnica comúnmente utilizada que asume que la polarización es uniforme en toda la fase ferroeléctrica, estrategia que, en efecto, subestima la influencia de las inclusiones en varios coeficientes piezoeléctricos.

A su vez, durante el desarrollo de este trabajo se ha encontrado que los potenciales utilizados para la densidad de energía almacenada pueden, bajo ciertos estados de carga, conducir a resultados que no son físicamente aceptables. Se presenta un análisis de la problemática y las condiciones bajo las cuales podría presentarse, y se discuten estrategias alternativas para obtener predicciones confiables para materiales compuestos ferroeléctricos.

Por otro lado y a pesar de que muchos de los materiales ferroeléctricos de interés son modelados como aislantes, éstos son en realidad semiconductores de banda prohibida ancha con una alta resistencia eléctrica y en los cuales las cargas libres y vacancias en la microestructura afectan de manera no trivial su comportamiento. Dada la complejidad de la problemática, se procede en este trabajo realizando una primera aproximación al tema, desarrollado una descripción multiescala para la respuesta electrostática de dieléctricos sólidos microestructurados que pueden experimentar polarización intrínseca y por cargas espaciales, pero sin tener en cuenta fenómenos ferroeléctricos. La polarización debido a cargas espaciales es considerada mediante la presencia de iones móviles que no pueden fluir a través de ciertas interfases microestructurales. Se obtienen predicciones para sistemas materiales sencillos, con el foco puesto en lograr una mejor comprensión del efecto de los iones móviles en el comportamiento del material. Se pretende de esta manera sentar las bases para futuros trabajos que expandan los modelos, incorporando el carácter semiconductor de los materiales ferrocerámicos. 


\section{Índice}

Resumen

$\begin{array}{ll}\text { Nomenclatura } & \text { ix }\end{array}$

1. Introducción $\quad 1$

1.1. Motivación . . . . . . . . . . . . . . . . . . 1

1.2. Ferroelectricidad en ferrocerámicos . . . . . . . . . . . . . . 5

1.3. Antecedentes y objetivos . . . . . . . . . . . . . . 7

1.4. Descripción del trabajo . . . . . . . . . . . . . . . . . . . . 13

1.5. Publicaciones derivadas de la tesis . . . . . . . . . . . . . . . 15

2. Teoría de medios continuos para el comportamiento electromecánico $\begin{array}{lr}\text { de ferrocerámicos } & 17\end{array}$

2.1. Introducción . . . . . . . . . . . . . . . . . . . . . 17

2.2. Marco teórico . . . . . . . . . . . . . . . . . . . 17

2.2.1. Sistema material . . . . . . . . . . . . . . 17

2.2.2. Ecuaciones de campo . . . . . . . . . . . . . . . . 18

2.2.3. Termodinámica y relaciones constitutivas . . . . . . . . . . . . 19

2.2.4. Relaciones constitutivas incrementales . . . . . . . . . . 21

2.2.5. Formas específicas de las funciones termodinámicas . . . . . . 22

2.3. Correspondencia con el ferromagnetismo . . . . . . . . . . . . 26

3. Evaluación de la teoría $\quad 29$

3.1. Introducción . . . . . . . . . . . . . . . . . . . . . 29

3.2. Predicciones para sólidos ferroeléctricos . . . . . . . . . . . . . . . . . 29

3.2.1. Estado de carga eléctrico uniaxial . . . . . . . . . . . 30

3.2.2. Estado de carga eléctrico multiaxial . . . . . . . . . . . . . 31

3.2.3. Estado de carga electromecánico uniaxial . . . . . . . . . . . 33

3.2.4. Estado de carga electromecánico multiaxial . . . . . . . . . . . 37

3.3. Predicciones para sólidos ferromagnéticos . . . . . . . . . . . 38

3.3.1. Estado de carga magnética uniaxial . . . . . . . . . . . . . . . 39

3.3.2. Estado de carga mecánico uniaxial . . . . . . . . . . . . . 40

3.4. Observaciones finales . . . . . . . . . . . . . . . . . . 42

4. Teoría multiescala para ferrocerámicos multifásicos 45

4.1. Introducción . . . . . . . . . . . . . . . . . 45

4.2. Formulación a dos escalas . . . . . . . . . . . . . . . 46

4.3. Modelo de laminados secuenciales para compuestos particulados . . . 49

5. Aplicación a ferrocerámicos porosos y con inclusiones $\quad 57$

5.1. Resultados para sistemas materiales representativos . . . . . . . . . . 57

5.2. Especímenes libres . . . . . . . . . . . . . . . . . 59

5.2.1. Ferrocerámicos con inclusiones metálicas . . . . . . . . . . 59

5.2.2. Ferrocerámico con microcavidades . . . . . . . . . . 65 
5.2.3. Ferrocerámicos con inclusiones ferromagnéticas . . . . . . . . 67

5.3. Especímenes bajo compresión . . . . . . . . . . . . . 71

6. Teoría multiescala para un dieléctrico con fases semiconductoras $\mathbf{7 5}$

6.1. Introducción . . . . . . . . . . . . . . . . . 75

6.2. Marco teórico . . . . . . . . . . . . . . . . . . . 75

6.2.1. Sistema material . . . . . . . . . . . . 75

6.2.2. Ecuaciones de campo . . . . . . . . . . . . . . 76

6.2.3. Termodinámica y relaciones constitutivas . . . . . . . . . . 77

6.2.4. Formulación Variacional . . . . . . . . . . . . . . . 78

6.3. Formulación multiescala . . . . . . . . . . . . . . . . . . . . 80

6.3.1. Formulación primaria . . . . . . . . . . . . . 80

6.3.2. Formulación dual . . . . . . . . . . . . . . . . . . . . 83

6.4. Cotas y estimaciones para la densidad de energía específica . . . . . . 84

6.4.1. Cotas de tipo Voigt . . . . . . . . . . . . . . . . . . 84

6.4.2. Cota de tipo Reuss . . . . . . . . . . . . . . . 85

6.4.3. Estimaciones basadas en medios lineales de comparación . . . 85

6.5. Especialización a densidades de energía particulares . . . . . . . . . . 89

6.5.1. Densidad de energía efectiva exacta . . . . . . . . . . . 89

6.5.2. Cotas de Voigt . . . . . . . . . . . . . . . . . . . . 91

6.5.3. Cota de Reuss . . . . . . . . . . . . . . . . . . . . 92

6.5.4. Estimaciones lineales de comparación . . . . . . . . . . . . 92

7. Aplicación a sistemas materiales modelo $\quad 95$

7.1. Introducción . . . . . . . . . . . . . . . . . . . 95

7.2. Materiales laminados . . . . . . . . . . . . . . . 95

7.2.1. Respuesta lineal . . . . . . . . . . . . . . . . . . 95

7.2.2. Respuesta no lineal . . . . . . . . . . . . . . . . . . . 99

7.3. Materiales particulados . . . . . . . . . . . . . . . . . 102

7.3.1. Partículas en suspensión diluida . . . . . . . . . . . . 102

7.3.2. Material con partículas con concentraciones arbitrarias . . . 106

8. Conclusiones y trabajo futuro 109

$\begin{array}{ll}\text { Referencias } & 113\end{array}$

Anexo A. Cálculos para materiales laminados con cargas libres $\quad \mathbf{1 2 7}$

A.1. Respuesta lineal . . . . . . . . . . . . . . . . . . . . . 127

A.2. Respuesta no lineal . . . . . . . . . . . . . . . . . . 128

Anexo B. Cálculos para materiales particulados con cargas libres $\mathbf{1 2 9}$

B.1. Matriz con partículas en suspensión diluida . . . . . . . . . . . . . . 129

B.2. Matriz con partículas en concentraciones arbitrarias . . . . . . . 130 


\section{Índice de figuras}

1.1. Efectos en los materiales . . . . . . . . . . . . . . . . 1

1.2. Ejemplos de aplicación de materiales electroactivos. . . . . . . . . . . 2

1.3. Dispositivos piezoeléctricos comerciales . . . . . . . . . . . . . 3

1.4. Microestructuras de materiales compuestos ferroeléctricos . . . . . . . 4

1.5. Orígenes microscópicos de la polarización eléctrica . . . . . . . . 5

1.6. Graficas experimentales del comportamiento de materiales ferroeléctricos 8

2.1. Dominio y condiciones de borde para un ferrocerámico . . . . . . . . 18

3.1. Comparación de las predicciones para especímenes ferroeléctricos para distintos intervalos de tiempo . . . . . . . . . . . . . . . 30

3.2. Representación esquemática del estado de carga eléctrico para los

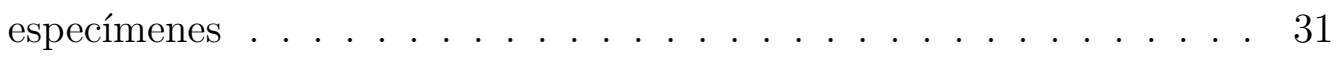

3.3. Predicciones para especímenes ferroeléctricos sin cargas mecánicas con exponentes de deformación irreversible $\gamma=1$ y $2 \ldots 32$

3.4. Curvas de rotación de la polarización . . . . . . . . . . . . . . 33

3.5. Representación esquemática del estado de carga electromecánico para los especímenes . . . . . . . . . . . . . . . . 34

3.6. Predicciones para especímenes ferroeléctricos sometidos a tracción longitudinal de 50MPa con exponentes de deformación irreversible $\gamma=1$ у $2 \ldots \ldots \ldots \ldots \ldots$

3.7. Predicciones para especímenes ferroeléctricos sometidos a compresión longitudinal de -50MPa con exponentes de deformación irreversible $\gamma=1$ y $2 \ldots \ldots \ldots \ldots \ldots \ldots$

3.8. Predicciones para especímenes ferroeléctricos sometidos a tracción perpendicular con exponentes de deformación irreversible $\gamma=1$ y 2 . 37

3.9. Predicciones para especímenes ferroeléctricos sometidos a compresión perpendicular con exponentes de deformación irreversible $\gamma=1$ y 2 . 38

3.10. Predicciones para especímenes sin cargas mecánicas con exponentes de deformación irreversible $\gamma=1$ y $2 \ldots$. . . . . . . . . . . 40

3.11. Predicciones para especímenes con exponente de deformación irreversible $\gamma=2$ sometidos a cargas mecánicas uniaxiales en ausencia de campos magnéticos . . . . . . . . . . . . . . . .

4.1. Dominio y condiciones de borde para un ferrocerámico con inclusiones. 47

4.2. Esquema de laminados bifásicos . . . . . . . . . . . . . . . . 50

5.1. Respuesta macroscópica de ferrocerámicos con partículas metálicas a diversas fracciones volumétricas . . . . . . . . . . . 60

5.2. Coeficientes piezoeléctricos macroscópicos de un ferrocerámico polarizado en función de la fracción volumétrica de partículas metálicas . . 61

5.3. Estadísticas de campo en ferrocerámicos con partículas metálicas . . . 63

5.4. Respuesta macroscópica de ferrocerámicos conteniendo microcavidades a diversas fracciones volumétricas . . . . . . . . . . . . . . . 
5.5. Coeficientes piezoeléctricos macroscópicos de un ferrocerámico polarizado en función de la porosidad . . . . . . . . . . . . . . . . 66

5.6. Estadísticas de campo en ferrocerámicos con microcavidades . . . . . 68

5.7. Respuesta macroscópica de un ferrocerámico con inclusiones ferromagnéticas . . . . . . . . . . . . . . . . . . . . . . 69

5.8. Deformación axial macroscópica y polarización irreversible promedio en la matriz de especímenes comprimidos . . . . . . . . . . . . . . 72

6.1. Dominio y condiciones de borde para un dieléctrico con varios microdominios . . . . . . . . . . . . . . 76

6.2. Linealización esquemática de la función $g^{(r)}(\mu)=\exp (\mu)-\mu-1$. . 87

7.1. Materiales laminados: celda unitaria y factor de amplificación . . . . 96

7.2. Predicciones para materiales laminados sometidos a campos eléctricos de baja intensidad . . . . . . . . . . . . . . . . 97

7.3. Predicciones para materiales laminados sometidos a campos eléctricos de intensidad arbitraria . . . . . . . . . . . . . . . 100

7.4. Materiales particulados: celda unitaria y factor de amplificación . . . 104

7.5. Predicciones para materiales particulados con concentración diluida . 105

7.6. Predicciones para materiales particulados con concentración arbitraria 107

\section{Índice de tablas}

3.1. Parámetros materiales adoptados para el sólido ferroeléctrico . . . . . 30

3.2. Parámetros materiales adoptados para el sólido ferromagnético . . . . 39

5.1. Parámetros materiales utilizados para el estudio de compuestos . . . 58 


\section{Nomenclatura}

\section{Acrónimos}

$\mathrm{BaTiO}_{3}$ Titanato de bario

BSZT Titanato zirconato de bario

EVR Elemento de volumen representativo

PVA Alcohol polivinílico

PZT Titanato zirconato de plomo

\section{Símbolos griegos}

$\alpha_{0} \quad$ Módulo piezoeléctrico axial $[N / C]$

$\alpha_{\perp} \quad$ Módulo piezoeléctrico lateral $[N / C]$

$\alpha=\quad$ Módulo piezoeléctrico de distorsión $[N / C]$

$\alpha_{0}^{(r)} \quad$ Propiedad de comparación [-]

$\beta_{0}^{(r)} \quad$ Propiedad de comparación [-]

$\boldsymbol{\beta} \quad$ Tensor de polarizabilidad magnética $\left[N / A^{2}\right]$

$\chi^{(r)} \quad$ Función característica [-]

$\Delta t \quad$ Paso de tiempo $[s]$

$\epsilon \quad$ Permitividad del sólido del sólido $\left[C^{2} / N \cdot m^{2}\right]$

$\epsilon_{0} \quad$ Permitividad del vacío $\left[C^{2} / N \cdot m^{2}\right]$

$\boldsymbol{\epsilon} \quad$ Tensor permitividad $\left[C^{2} / N \cdot m^{2}\right]$

$\varepsilon \quad$ Tensor de deformaciones [-]

$\kappa \quad$ Polarizabilidad $\left[N \cdot m^{2} / C^{2}\right]$

$\kappa \quad$ Tensor polarizabilidad $\left[N \cdot m^{2} / C^{2}\right]$

$\lambda \quad$ Constante de Lamé $\left[N / m^{2}\right]$

$\mu \quad$ Constante de Lamé $\left[N / m^{2}\right]$

$\mu_{+} \quad$ Potencial electroquímico de los cationes móviles $[\mathrm{J} / \mathrm{mol}]$

$\breve{\mu}^{(r)} \quad$ Valor óptimo de $\mu$ para las propiedades de comparación $\alpha_{0}^{(r)}$ y $\beta_{0}^{(r)}[-]$

$\hat{\mu}^{(r)} \quad$ Valor óptimo de $\mu$ para las propiedades de comparación $\alpha_{0}^{(r)}$ y $\beta_{0}^{(r)}[-]$

$\Omega \quad$ Dominio ocupado por el espécimen $[-]$

$\Omega^{(r)} \quad$ Dominio ocupado por el microdominio $r[-]$

$\partial \Omega \quad$ Borde del espécimen [-] 
$\partial \Omega_{e} \quad$ Borde del espécimen con electrodos [-]

$\partial \Omega_{u} \quad$ Borde del espécimen con desplazamiento impuesto [-]

$\phi \quad$ Campo de potencial electrostático $[V]$

$\bar{\phi} \quad$ Potencial electrostático macroscópico $[V]$

$\hat{\phi}_{e} \quad$ Potencial eléctrico aplicado en los electrodos $[V]$

$\varphi \quad$ Potencial de disipación $\left[\mathrm{J} / \mathrm{m}^{3}\right]$

$\psi \quad$ Densidad de energía complementaria $\left[\mathrm{J} / \mathrm{m}^{3}\right]$

$\rho_{c} \quad$ Densidad volumétrica de carga eléctrica $\left[C / \mathrm{m}^{3}\right]$

$\sigma_{c} \quad$ Densidad de cargas eléctrica $\left[\mathrm{C} / \mathrm{m}^{2}\right]$

$\sigma_{f} \quad$ Densidad superficial de cargas libres $\left[C / \mathrm{m}^{2}\right]$

$\boldsymbol{\sigma} \quad$ Tensor de tensiones $[P a]$

$\Xi \quad$ Elemento de volumen representativo

\section{Símbolos romanos}

B Vector de inducción magnética [Tesla]

$c_{+} \quad$ Concentración molar de cationes [-]

$c_{-} \quad$ Concentración molar de contraiones [-]

$\mathbb{C} \quad$ Tensor elasticidad $\left[N / m^{2}\right]$

$d_{i j} \quad$ Coeficiente piezoeléctrico $[\mathrm{m} / \mathrm{V}]$

$\mathrm{d} V \quad$ Diferencial de volumen $\left[\mathrm{m}^{3}\right]$

D Vector de desplazamiento eléctrico $\left[C / \mathrm{m}^{2}\right]$

$\mathcal{D} \quad$ Disipación $[J]$

$\mathfrak{e} \quad$ Densidad de energía libre de Helmholtz $\left[\mathrm{J} / \mathrm{m}^{3}\right]$

$\mathfrak{e}^{*} \quad$ Densidad de energía complementaria $\left[\mathrm{J} / \mathrm{m}^{3}\right]$

$\mathfrak{e}_{p d} \quad$ Energía almacenada debido a los dipolos permanentes $\left[\mathrm{J} / \mathrm{m}^{3}\right]$

$\mathfrak{E} \quad$ Energía total del sistema material y sus alrededores $[J]$

E Vector de intensidad de campo eléctrico $[N / C]$

$f^{(r)} \quad$ Fracción volumétrica de la celda unitaria $\Omega_{\#}$ ocupada por $\Omega_{\#}^{(r)}[-]$

F Constante de Faraday $[C / \mathrm{mol}]$

$g_{i j} \quad$ Coeficiente piezoeléctrico $[V \cdot m / N]$

$h_{0} \quad$ Campo magnético de referencia $[A / m]$

$h_{c} \quad$ Campo magnético coercitivo $[A / m]$

h Tensor piezoeléctrico $[N / C]$

H Vector de intensidad del campo magnético $[A / m]$ 
I Tensor identidad de segundo orden [-]

I Tensor identidad de cuarto orden $[-]$

$m_{s} \quad$ Magnetización de saturación $[N / A \cdot m]$

$\dot{m}_{0} \quad$ Tasa de magnetización $[N \cdot s / A \cdot m]$

m Vector de magnetización irreversible $[N / A \cdot m]$

$M \quad$ Rango del laminado secuencial [-- ]

M Magnetización del sólido $[N / A \cdot m]$

$n \quad$ Exponente que caracteriza la sensibilidad a la tasa [-]

n Normal a la superficie

$p_{s} \quad$ Polarización de saturación $\left[C / m^{2}\right]$

$\mathbf{P} \quad$ Campo de polarización intrínseca $\left[\mathrm{C} / \mathrm{m}^{2}\right]$

$R \quad$ Constante de gas universal $[\mathrm{J} / \mathrm{K} \cdot \mathrm{mol}]$

S Tensor flexibilidad $\left[\mathrm{m}^{2} / N\right]$

$t \quad$ Instante de tiempo $[s]$

$\hat{\mathbf{t}} \quad$ Tracción impuesta en el borde del espécimen $[\mathrm{Pa}]$

$T \quad$ Temperatura absoluta $[K]$

$\bar{u} \quad$ Densidad de energía efectiva complementaria $\left[\mathrm{J} / \mathrm{m}^{3}\right]$

$\bar{u}_{0} \quad$ Energía efectiva del medio lineal de comparación $\left[\mathrm{J} / \mathrm{m}^{3}\right]$

$u_{0}^{(r)} \quad$ Potencial lineal de comparación del microdominio $r\left[\mathrm{~J} / \mathrm{m}^{3}\right]$

$\mathbf{u} \quad$ Vector de campo de desplazamiento $[m]$

$\hat{\mathbf{u}} \quad$ Desplazamiento impuesto en el borde del espécimen $[m]$

$v^{(r)} \quad$ Función desplazada [-]

$Y \quad$ Módulo de Young del sólido $\left[\mathrm{N} / \mathrm{m}^{2}\right]$

$z \quad$ Valencia $[-]$

\section{Símbolos matemáticos}

$\langle\cdot\rangle \quad$ Promedio volumétrico en $\Xi$

$\nabla \quad$ Operador Nabla

$\otimes_{s} \quad$ Parte simétrica del producto tensorial

$-\quad$ Valor promedio en $\Xi$

\section{Superíndices}

$r$

Correspondiente a la fase $r$ 
Facultad de Ingeniería, UNLP 


\section{Introducción}

\subsection{Motivación}

En la actualidad, muchos problemas de ingeniería de diversos campos se resuelven utilizando los denominados sistemas inteligentes, es decir, sistemas adaptativos o controlados. En lo que respecta al aspecto de sensado y actuación de estos sistemas, existen una gran variedad de fenómenos físicos y materiales para cumplir con su implementación. En un sentido general, todos los materiales pueden considerarse como un sistema que, frente a la aplicación de estímulos externos, puede responder de maneras distintas (figura 1.1). Pueden encontrarse entonces materiales que tienen respuestas triviales frente a la aplicación de estímulos externos, como por ejemplo los materiales conductores o elásticos, que generan una corriente eléctrica o una deformación frente a la aplicación de un voltaje o una tensión mecánica, respectivamente. Existen a su vez, materiales como los piroeléctricos o piezoeléctricos que generan un campo eléctrico al ser sometidos al calor o a una tensión mecánica, respectivamente.

Un material inteligente puede definirse, sin embargo, como aquel que posee una o más propiedades que pueden ser modificadas de manera controlada y reversible mediante la aplicación de un estímulo externo. El concepto de un material que pueda adaptar su funcionalidad dependiendo del entorno se basa en la posibilidad de integrar actuadores, sensores y controladores con materiales y componentes estructurales en un sistema material inteligente.

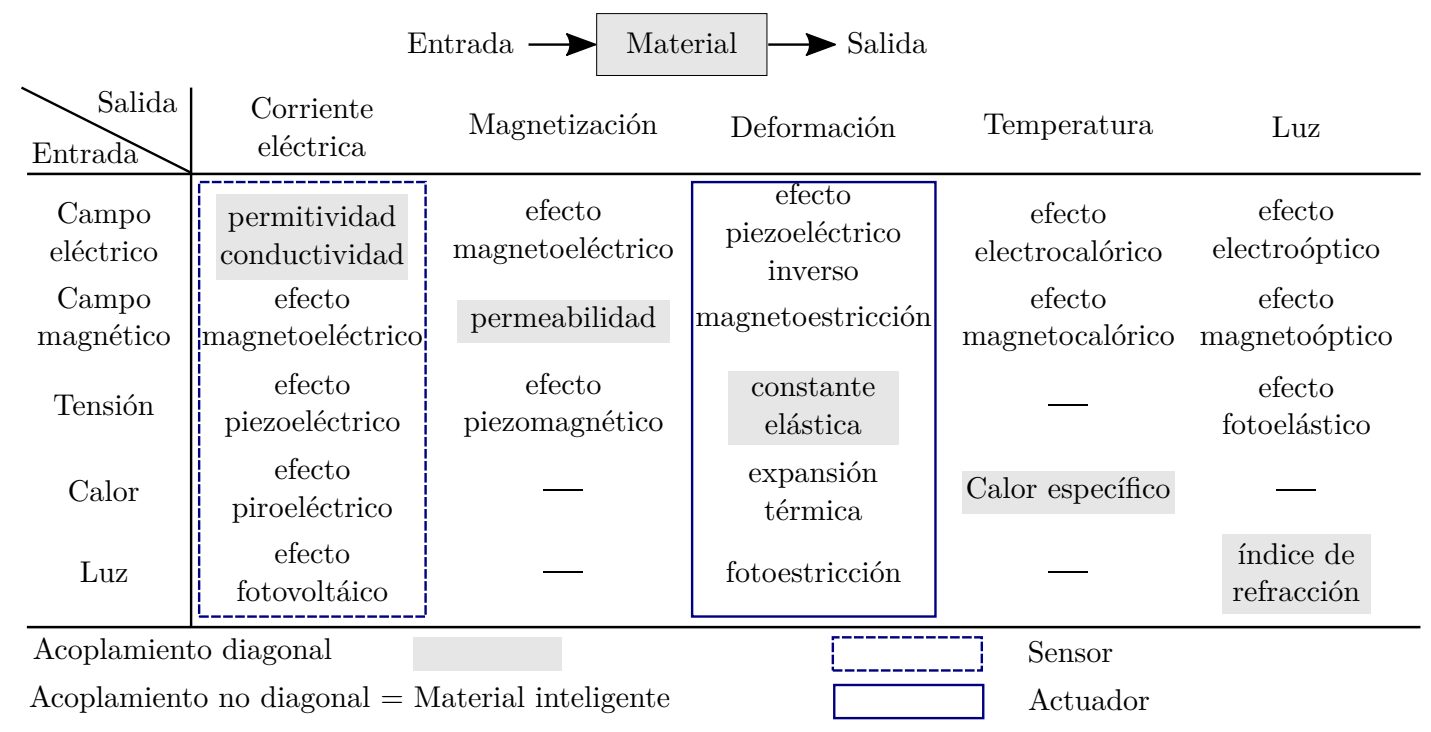

Figura 1.1: Efectos en los materiales (Uchino, 2010). 
a)

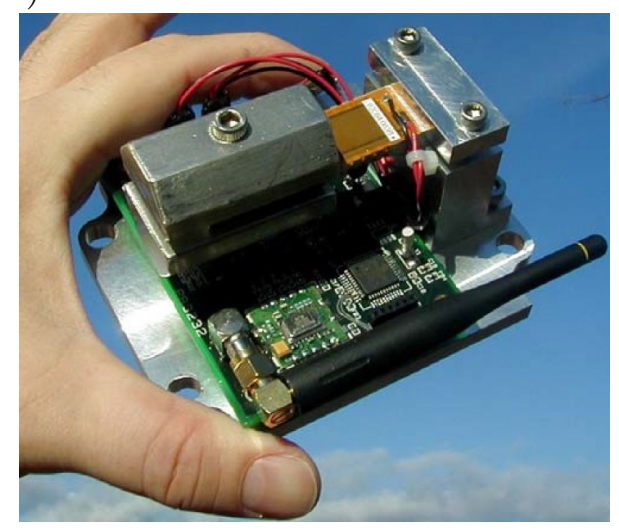

b)

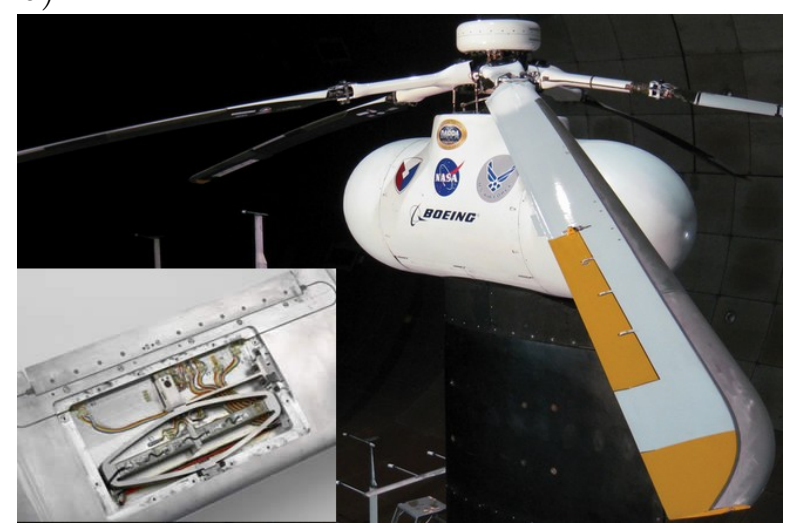

Figura 1.2: Ejemplos de aplicación de materiales electroactivos. a) Uso en la generación de energía por vibraciones en módulo de sensado autónomo (Arms et al., 2005); b) Uso como actuador en palas de helicóptero, con detalle en las características del actuador.

La elección entre la variedad de materiales y fenómenos disponibles para resolver un problema particular dependerá de las necesidades técnicas, costos, y disponibilidad de cada material. Los materiales piezocerámicos son, en general, muy buenos candidatos para aplicaciones que requieren tiempos de respuestas muy cortos, posicionamiento de alta precisión y niveles de fuerza de actuación considerables en sistemas de geometría compleja. Se entiende aquí como piezocerámico a un material ferroeléctrico policristalino utilizado para aplicaciones de ingeniería en su rango piezoeléctrico reversible. En efecto, la mayoría de los materiales utilizados en aplicaciones tecnológicas electromecánicas son ferroeléctricos. Algunos ejemplos prácticos de aplicación de estos materiales en el ámbito aeronáutico pueden observarse en la figura 1.2, donde se presenta un módulo sensor que aprovecha las vibraciones del ambiente para generar energía a partir de la deformación de un material ferroeléctrico, y también donde se utilizan como actuadores en superficies de control de una pala de helicóptero.

En un intento de ajustarse a las necesidades que las diversas aplicaciones puedan tener, los materiales ferroeléctricos monolíticos se presentan comercialmente en forma de láminas delgadas o conformados en discos, anillos, barras, entre otras geometrías (figura 1.3a). Ahora bien, los procesos de manufactura típicos para obtener un dispositivo ferroeléctrico con una composición determinada se dividen en dos etapas: primeramente se obtiene un polvo cerámico que luego es sinterizado en la geometría deseada. La composición del polvo se ajusta a modo de obtener, luego del proceso de fabricación, un cerámico con una estructura del tipo perovskita, lo que asegura sus características ferroeléctricas. Para obtener el polvo cerámico (véase por ejemplo, Kawada et al., 2010; Tanaka et al., 2012), se parte de algunas de las materias primas de los materiales constitutivos del óxido de perovskita, que son mezclados y molidos. Estas materias primas pueden provenir de óxidos, carbonatos, sales orgánicas ácidas, alcóxidos o compuestos similares de los elementos constitutivos. 
a)

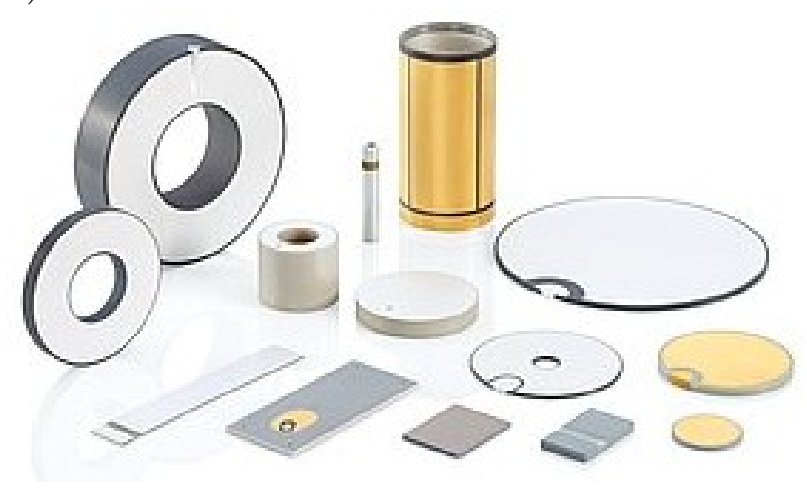

b)

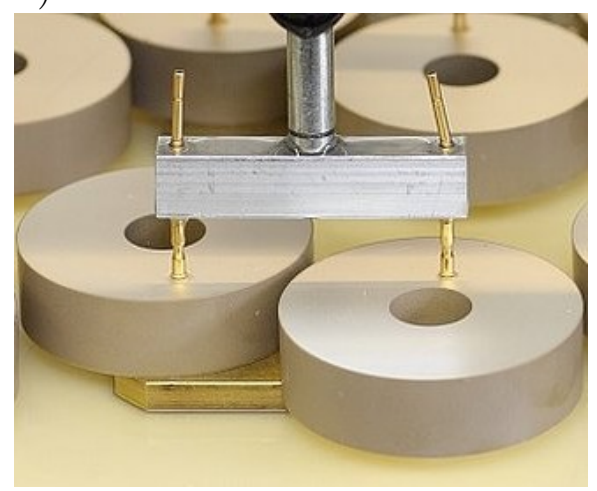

Figura 1.3: Dispositivos piezoeléctricos comerciales. a) Variedad de configuraciones geométricas disponibles. b) Ejemplo de proceso de polarización. (PI Piezo Technology, 2017).

El proceso de mezclado y molido puede realizarse utilizando un molino de bolas, o si en cambio se utiliza un proceso húmedo, se debe utilizar un solvente como medio de dispersión, que luego puede removerse por evaporación o filtrado. Luego, esta mezcla de materiales es calcinada, lográndose que las materias primas de los elementos constitutivos reaccionen y se combinen para obtener polvo de óxido de perovskita. Alternativamente, el polvo puede sintetizarse en fase líquida mediante las técnicas de sol-gel o síntesis hidrotérmica. Dependiendo del tipo de compuesto que se desea obtener, puede realizarse un segundo proceso de mezclado del polvo obtenido con nuevos aditivos, el cual es sometido a un nuevo proceso de calcinación para obtener el polvo de óxido de perovskita final.

Una vez obtenido el polvo cerámico de óxido de perovskita es posible sintetizar el dispositivo en la forma deseada. El moldeo del dispositivo puede llevarse a cabo mediante procesos de extrusión, moldeo por inyección, a presión o en cinta, vaciado, prensado isostático en frío, entre otros. La cocción del cuerpo moldeado puede llevarse a cabo mediante horneado en atmósfera común o a presión, como son el prensado con calor o el proceso de compresión isostática en caliente. Cabe destacar que en el proceso de cocción del cerámico se utilizan temperaturas del orden de los $1000^{\circ} \mathrm{C}$, muy por encima de la temperatura de Curie del material, por lo que se espera que luego del proceso de enfriamiento el dispositivo no tenga una polarización remanente neta. Este fenómeno será discutido en mayor detalle en la próxima sección.

Ya contando con el cerámico cocido y dependiendo de la aplicación, se agregan electrodos conductivos en su superficie mediante técnicas como la impresión serigráfica o la pulverización (sputtering). Por último y teniendo en cuenta que el cerámico resultante no posee una polarización neta, se procede a la polarización del mismo mediante la aplicación de un campo eléctrico (figura 1.3b). El esquema de polarización puede variar, y existen diversos estudios que han evaluado el efecto de la temperatura 
a)

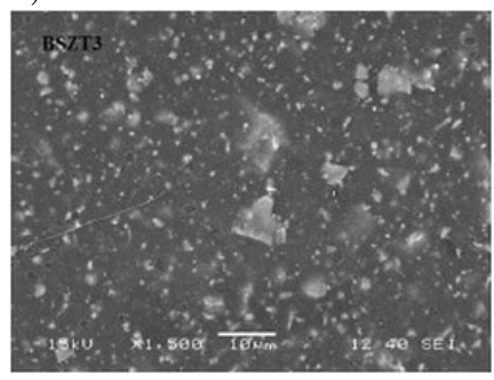

b)

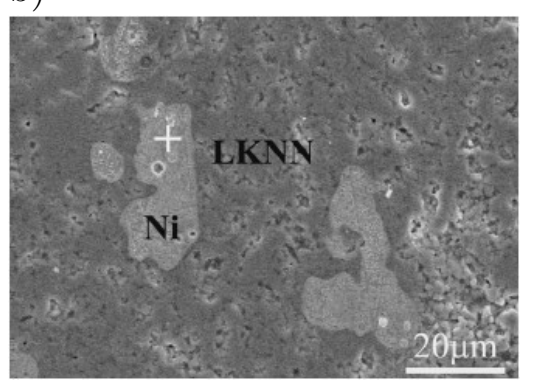

c)

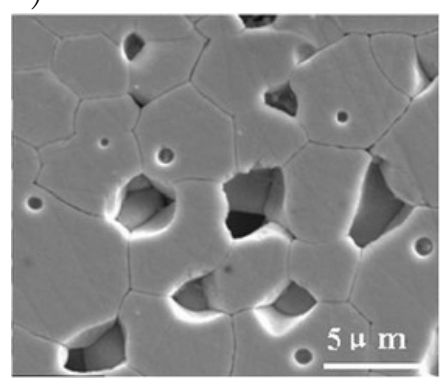

Figura 1.4: a) Compuesto de matriz polimérica de alcohol polivinílico (PVA) e inclusiones de titanato zirconato de bario (BSZT) (Senthil et al., 2012). b) Compuesto de matriz ferroeléctrica de LKNN e inclusiones de níquel (Zhang et al., 2010a). c) Matriz ferroeléctrica de titanato zirconato de plomo (PZT) con poros (Nie et al., 2010).

(Zhang et al., 2010b, 2011) o de las tensiones mecánicas (Kumazawa et al., 1998; Rödel et al., 2007) para optimizar proceso.

Ahora bien, en vistas de optimizar los sistemas materiales para aplicaciones específicas, ha surgido la necesidad de contar con materiales electrodeformables con propiedades no disponibles en materiales ferroeléctricos monolíticos. Esto ha motivado el desarrollo de una creciente variedad de materiales compuestos ferroeléctricos bifásicos con microestructuras de tipo matriz-inclusión. Tal es el caso de matrices poliméricas no polares con inclusiones micrométricas de cerámicos ferroeléctricos (por ejemplo, Capsal et al., 2012; Lam et al., 2005; Olmos et al., 2012), de matrices cerámicas ferroeléctricas con inclusiones metálicas (por ejemplo, Duan et al., 2000; Ning et al., 2012; Takagi et al., 2003; Zhang et al., 2010a), y de matrices poliméricas ferroeléctricas con inclusiones cerámicas ferroeléctricas (por ejemplo, Mao et al., 2010; Petchsuk et al., 2011). Incluso se han desarrollado cerámicos ferroeléctricos con porosidad controlada (por ejemplo, Piazza et al., 2010). También se ha explorado el desarrollo de sistemas multiferróicos capaces de exhibir acoplamiento magnetoeléctrico. En su definición más general, se denomina como efecto magnetoeléctrico en los materiales al acoplamiento entre campos eléctricos y magnéticos (Fiebig, 2005). Dado que este fenómeno suele ser extremadamente débil en materiales monofásicos, se está estudiando la posibilidad de mejorar su comportamiento a partir de materiales compuestos multifásicos (Zhai et al., 2008). En materiales compuestos entonces, el efecto magnetoeléctrico surge gracias a la combinación de dos clases de propiedades materiales, como ser la magnetoestricción y la piezoelectricidad. Al aplicar un campo magnético, la fase magnetoestrictiva produce deformaciones que son luego transferidas a la fase piezoeléctrica que convierte esas deformaciones en carga eléctrica. Esta conversión de campos magnéticos a campos eléctricos se denomina efecto magnetoeléctrico inverso (Nan et al., 2008). Si bien el modelado de materiales ferromagnéticos y multifuncionales excede los alcances de este trabajo, realizando mínimos cambios es posible adaptar los modelos y herramientas 
desarrollados para compuestos bifásicos ferroeléctricos para obtener estimaciones para este otro tipo de materiales. Esto permite evaluar la potencial aplicación de las herramientas desarrolladas aquí en otras áreas de interés tecnológico.

A continuación se describen brevemente los fenómenos físicos que dan lugar al acoplamiento electromecánico en los materiales ferroeléctricos.

\subsection{Ferroelectricidad en ferrocerámicos}

Típicamente, los materiales ferroeléctricos son considerados dieléctricos, es decir, aislantes que no pueden conducir una corriente eléctrica. Los materiales dieléctricos poseen cargas ligadas que, aunque no pueden fluir libremente por el material, pueden moverse levemente respecto de su posición original ante la presencia de campos eléctricos externos. Este fenómeno se conoce como polarización eléctrica y pueden definirse tres contribuciones primarias al mismo: electrónica, iónica y relacionada a la reorientación de dipolos. En la figura 1.5 se presenta esquemáticamente cada uno de estos mecanismos, que contribuirán a la polarización total del material en distinta proporción dependiendo de la frecuencia del campo aplicado.

La estructura microscópica de los materiales piezocerámicos es de naturaleza cristalina. La misma se encuentra dividida en granos en los cuales se tiene una red cristalina con una orientación determinada y formada por una repetición periódica de una celda unitaria. Esta celda unitaria es un agrupamiento de iones cagados positiva y negativamente y que dependen del material específico. Dependiendo de la estructura cristalina del material, los centros de cargas positivas y negativas pueden no coincidir, incluso ante la ausencia de campos eléctricos externos. Se dice entonces que este tipo de cristales poseen una polarización espontánea. En particular, cuando la dirección de la polarización espontánea puede cambiarse aplicando un campo eléctrico externo, los materiales se denominan ferroeléctricos. La ferroelectricidad se refiere entonces a

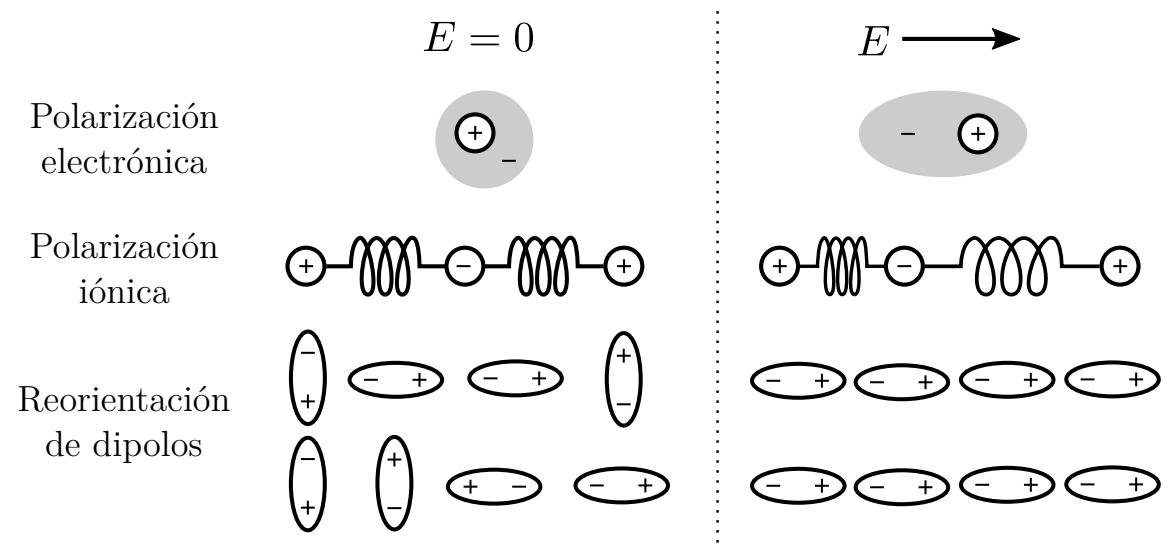

Figura 1.5: Orígenes microscópicos de la polarización eléctrica (Uchino, 2010). 
la capacidad de ciertos dieléctricos polares de mantener una polarización eléctrica permanente que puede ser alterada mediante la aplicación de un campo eléctrico externo (Lines y Glass, 1977). Debido a que el cambio en la polarización suele estar acompañado de una deformación mecánica, los materiales ferroeléctricos son electrodeformables, y como tales, pueden utilizarse como sensores y actuadores (por ejemplo, Capsal et al., 2012; Xu, 1991), recolectores de energía (por ejemplo, van den Ende et al., 2012), amortiguadores materiales (por ejemplo, Asare et al., 2012) y otros microdispositivos.

El titanato de bario $\left(\mathrm{BaTiO}_{3}\right)$ y el titanato zirconato de plomo $\left(\mathrm{PbZr}_{x} \mathrm{Ti}_{1-x} \mathrm{O}_{3}\right)$ son quizás dos de los materiales ferroeléctricos más utilizados. Sin embargo, la ferroelectricidad puede encontrarse tanto en materiales cerámicos como poliméricos. En todos los casos y como ya fue mencionado, se trata de materiales policristalinos que al ser enfriados por debajo de una temperatura crítica - denominada temperatura de Curie - sufren una transformación de fase paraeléctrica a ferroeléctrica. Inicialmente, la fase ferroeléctrica contiene una distribución aleatoria de polarización permanente a nivel microscópico, y en consecuencia no exhibe una polarización permanente neta a nivel macroscópico. Como ya fue mencionado, para que esto último ocurra, el material ferroeléctrico debe polarizarse, lo que resulta ser un proceso fuertemente disipativo y que requiere de herramientas sofisticadas para evaluar su efecto en el desempeño de los materiales.

Ahora bien, a pesar de que muchos de los materiales ferroeléctricos de interés son modelados como aislantes, éstos son en realidad semiconductores de banda prohibida ancha con una alta resistencia eléctrica y en los cuales las cargas libres y vacancias en la microestructura afectan de manera no trivial su comportamiento (Xiao y Bhattacharya, 2008). Defectos en la red cristalina como átomos de impurezas y vacancias tienden a acumularse en los bordes de grano del cerámico dieléctrico (Priester, 2013; Randle y Owen, 2006), favoreciendo la conductividad eléctrica. El efecto de la conductividad eléctrica en la dispersión y atenuación de ondas acústicas en materiales semiconductores piezoeléctricos es un fenómeno bien conocido y estudiado (Auld, 1969; Wauer y Suherman, 1997; Yang y Zhou, 2005). Por lo tanto, además de los diversos micromecanismos concomitantes de origen diverso que definen la polarización macroscópica de un dieléctrico sólido, cuando un espécimen posee una microestructura fina, el movimiento de los defectos cargados estabilizan cierta configuración de dominio generándose un campo eléctrico residual que se opone al cambio de la polarización cuando se aplica un campo eléctrico externo. Esto implica que la polarización intrínseca de origen atómico y molecular puede verse amplificada significativamente por una polarización extrínseca producida por cargas espaciales en el entorno de las interfaces microestructurales que bloquean el flujo de iones. Esta 
polarización por cargas espaciales suele ocasionar fenómenos de dispersión dieléctrica a baja frecuencia y efectos de talla microestructural (véase, por ejemplo, Boersma y van Turnhout, 1998; Mackey et al., 2012; Tonkoshkur et al., 2017). Recientemente, este fenómeno ha sido propuesto como un posible micromecanismo responsable del mejoramiento de las características dieléctricas observadas en algunos polímeros nanorellenos y en cerámicos del tipo perovskita (por ejemplo, Brizé et al., 2006; Lopez-Pamies et al., 2014; Zang et al., 2005; Zhou et al., 2016). Se ha observado también que la fatiga y la ruptura dieléctrica de los cerámicos ferroeléctricos típicos dependen del tipo de electrodo utilizado (de Araujo et al., 1995; Ramesh et al., 1992; Scott, 2000). Este fenómeno se debe a que la naturaleza semiconductora del material favorece la aparición de una región con reducción de electrones en la cercanía de la interfaz ferroeléctrico-electrodo, lo que depende de la combinación particular de materiales. Además, la presencia de cargas libres puede influir en el envejecimiento del material, desarrollándose un efecto de memoria de un patrón de dominios en el cual se ha mantenido por un largo tiempo (Ren, 2004).

\subsection{Antecedentes y objetivos}

Anteriormente se ha mencionado que el proceso de polarización macroscópica de un material ferroeléctrico es fuertemente disipativo. Esto queda en evidencia en la figura 1.6a, donde se muestra la polarización en función del campo eléctrico aplicado en una muestra de PZT. La respuesta manifiesta, en efecto, una gran histéresis y la principal fuente de disipación radica en el cambio de polarización permanente microscópica como consecuencia de ciertos procesos irreversibles ocurridos a nivel atómico (por ejemplo, Damjanovič, 2006). Una vez polarizado, el material se vuelve piezoeléctrico, de manera tal que la acción de un campo eléctrico externo moderado produce una pequeña deformación directamente proporcional a la intensidad del campo.

Ahora bien, en materiales compuestos que se sintetizan a temperaturas superiores a la temperatura de Curie y luego se polarizan a temperaturas inferiores a esta, es esperable que como consecuencia de la heterogeneidad microestructural y el acoplamiento electromecánico, la intensidad de campo eléctrico presente dentro de un compuesto durante el proceso de polarización exhiba fuertes variaciones espaciales y pueda generar tensiones internas significativas. Es así que el material compuesto está localmente sometido a cargas electromecánicas incluso si la carga macroscópica es puramente eléctrica. Este tipo de respuesta es compleja y en la figura 1.6 se muestra la polarización en función del campo eléctrico aplicado a diversos cerámicos ferroeléctricos con inclusiones o poros, sometidos a cargas eléctricas cíclicas. Se observa que el compuesto posee un comportamiento disipativo que tiene su origen en 
a)

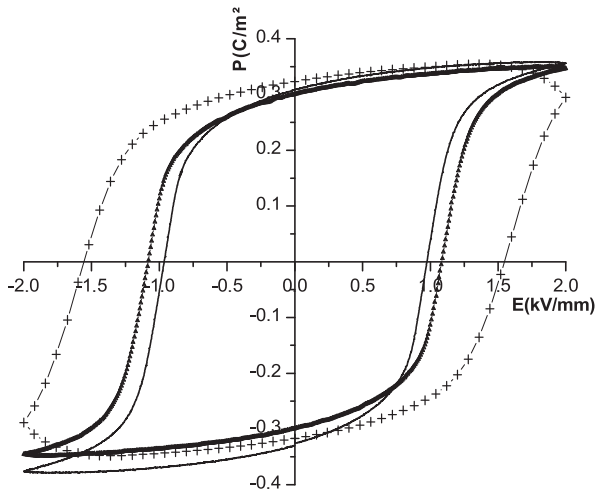

c)

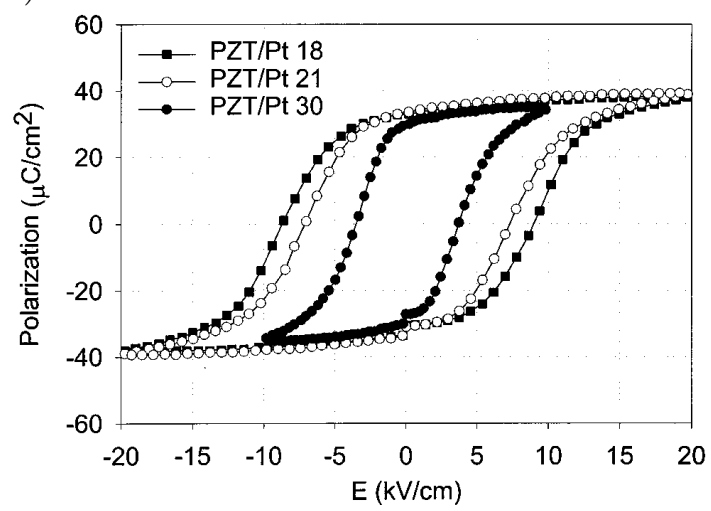

b)

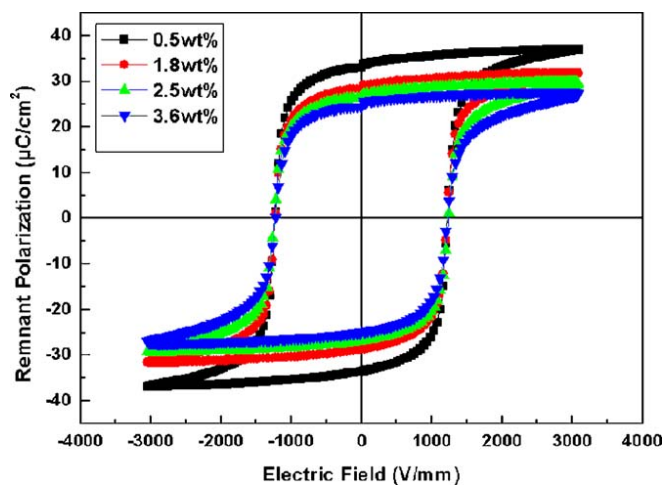

d)

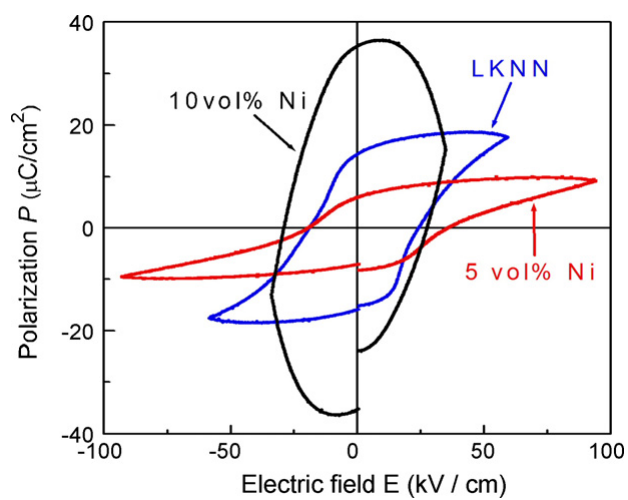

Figura 1.6: Polarización versus campo eléctrico aplicado en: a) un PZT bajo cargas eléctricas cíclicas de varias frecuencias (Miehe y Rosato, 2011); b) PZT95/5 con distintos niveles de porosidad (Nie et al., 2010); c) un PZT con distintas concentraciones de platino (Duan et al., 2000); d) LKNN con distintas concentraciones de níquel (Zhang et al., 2010a).

la fuerte histéresis eléctrica del PZT, que a su vez depende de la frecuencia del campo eléctrico aplicado. Además, el comportamiento macroscópico puede variar según la inclusión de modo que, por ejemplo, el agregado de microcavidades (figura 1.6b) no produce cambios en el valor del campo eléctrico coercitivo pero si afecta la magnitud de la polarización remanente del compuesto. Por otro lado, el agregado de inclusiones metálicas (figuras 1.6c,d) produce el efecto contrario. En este caso incluso puede ocurrir que la respuesta del compuesto cambie radicalmente, como se observa en la figura 1.6d en donde ni siquiera se obtiene un ciclo de histéresis cerrado. Este fenómeno puede explicarse por la presencia de cargas libres en la matriz aportadas por las inclusiones (Suryanarayana y Bhattacharya, 2012). Más aún, cuando el estado de cargas involucra solicitaciones tanto eléctricas como mecánicas, el comportamiento del compuesto depende también de la histéresis mecánica de los materiales constitutivos. Caracterizar experimentalmente respuestas electromecánicas hereditarias bajo historias de carga generales en función de la microestructura es pues impracticable y en consecuencia resulta necesario desarrollar modelos micromecánicos capaces de predecir con aceptable precisión la influencia de segundas fases en la 
respuesta electromecánica de materiales ferroeléctricos. Este es el objetivo general de este trabajo.

A partir de lo expuesto, surge que una descripción precisa de la respuesta electromecánica de compuestos ferroeléctricos exige modelos constitutivos que tengan en cuenta $(i)$ las no linealidades constitutivas, $(i i)$ la compleja distribución aleatoria de la microestructura, (iii) el acoplamiento electromecánico y (iv) la histéresis tanto eléctrica como mecánica de los materiales constitutivos. En este aspecto pueden identificarse dos grandes áreas para encarar el modelado constitutivo del material: el modelado fenomenológico y el modelado micromecánico (véase, por ejemplo, Laskewitz y Kamlah, 2010).

Los modelos fenomenológicos para estimar la respuesta de materiales resultan ser muy eficientes para describir el complejo comportamiento de los materiales ferrocerámicos. $\mathrm{Su}$ principal desventaja surge del hecho que la respuesta del material se describe fenomenológicamente, lo que implica que necesitan ser calibrados para una condición de carga determinada. Sin embargo, fuera del rango considerado durante el desarrollo del modelo, existe el riesgo de que sus predicciones sean inexactas, o incluso de que sean cualitativamente erróneas.

En esta rama, y desde los primeros trabajos de Chen (1980), se han hecho grandes avances en el campo del modelado fenomenológico de materiales ferroeléctricos, describiéndose a continuación algunas de las contribuciones más relevantes. McMeeking y Landis (2002) propusieron un modelo fenomenológico para la conmutación de dominios ferroeléctricos en materiales sometidos a estados de carga electromecánicos multiaxiales. El mismo se basa en la teoría de endurecimiento cinemático, con una superficie de conmutación en el espacio de los campos eléctricos y de tensiones. La deformación permanente se expresa como una función de la polarización permanente. Si bien este enfoque simplifica notablemente el modelo, excluye comportamientos que son únicamente ferroelásticos. Esta restricción no aplica al modelo de Landis (2002) que introduce una superficie de conmutación de dominios y reglas de flujo para las variables internas en el espacio de campos eléctricos y de tensiones.

Huber y Fleck (2001) propusieron un modelo fenomenológico que se centra mayormente en estados de carga cíclicos uniaxiales. Por otro lado, Kessler y Balke (2001) derivaron una expresión para la tasa de liberación de energía local y promedio durante procesos de orientación de la polarización. A partir de esta base, se desarrolló un modelo de repolarización que permite describir la rotación pura de la polarización para una magnitud dada. Schröder y Gross (2004) desarrollaron una formulación electromecánica acoplada para materiales transversalmente isótropos con comportamiento reversible. Presentaron tanto una formulación variacional como 
de elementos finitos para problemas acoplados en pequeñas deformaciones. Luego Schröder y Romanowski (2005) mejoraron este modelo con respecto a la conmutación de dominios de modo que sea capaz de describir las características fundamentales de los efectos de histéresis. Sin embargo, solo la magnitud de la polarización puede cambiar mientras su dirección permanece fija en el espacio, y la deformación es únicamente una función de la polarización remanente.

Belov y Kreher (2005) propusieron dos modelos de tipo viscoplástico sin condiciones de carga para el comienzo de la conmutación de dominios. La evolución de los dominios se describe mediante ecuaciones dependientes de la tasa para las fracciones volumétricas de las distintas variantes de orientaciones. El primer modelo permite seis orientaciones de dominios diferentes y fue propuesto para estados de carga uniaxiales. El segundo modelo tiene cuarenta y dos orientaciones de dominios diferentes y puede utilizarse para simulaciones con estados de carga multiaxiales.

Klinkel (2006) desarrolló un modelo unidimensional para piezocerámicos con efectos de histéresis ferroeléctricos y ferroelásticos utilizando un enfoque termodinámicamente consistente. Utiliza la energía libre de Helmholtz y una superficie de conmutación de dominios para el marco termodinámico de su trabajo. Este modelo tiene la característica de introducir, además de la deformación irreversible, un campo eléctrico irreversible como variable de estado. El objetivo es simplificar la implementación del modelo en elementos finitos pero no tiene una interpretación física inmediata.

Alternativamente, muchos trabajos teóricos están basados en el modelado micromecánico teniendo en cuenta el carácter policristalino del ferrocerámico, lo que ha ayudado a tener un mejor entendimiento sobre el comportamiento de los materiales ferroeléctricos. Estos poseen un gran número de ventajas comparados con los modelos fenomenológicos debido a que su rango de validez es más general porque se tienen en cuenta mecanismos básicos a nivel microscópico. En esta línea de estudio y basándose en el comportamiento constitutivo de monocristales, Chen y Lynch (1998) desarrollaron un modelo micromecánico para cerámicos ferroeléctricos policristalinos. Este modelo simula estructuras cristalinas tetragonales y romboidales en las cuales se implementa una saturación del efecto piezoeléctrico lineal y tiene en cuenta la interacción entre distintos granos del policristal. Por su parte, Huber et al. (1999) y Huber y Fleck (2004) describieron un modelo micromecánico basado en la teoría de plasticidad de cristales. Se define a un grano como una mezcla de variantes, siendo cada variante una representación de todos los dominios con igual orientación de la polarización espontánea dentro del grano. Los procesos de conmutación de dominios transforman una variante en otra y las propiedades del policristal se obtienen mediante un esquema autoconsistente, dando como resultado la polarización y deformación irreversibles, así como las propiedades electromecánicas promedio. En el trabajo de 
Kamlah et al. (2005), la interacción entre granos se modela explícitamente por medio del método de los elementos finitos. Seemann et al. (2004) y Delibas et al. (2006) derivaron un modelo a nivel de granos de un policristal en el cual se asume que, luego de la polarización, todos los dominios del grano tienen la misma orientación. La base de este modelo es la aproximación energética de Hwang y McMeeking (1998) en la cual se considera la energía potencial de cada dominio de modo tal que si su energía potencial crece más allá de cierto límite, el dominio conmuta. La respuesta del policristal es el promedio de todos los procesos en los distintos dominios. La interacción mutua entre dominios se considera a través de un conjunto de funciones probabilísticas. Smith et al. (2003) desarrollaron un modelo micromecánico en base a las energías libres de Helmholtz y Gibbs a nivel de la red cristalina de un monocristal. Para embeber estos monocristales en estructuras policristalinas se realiza una homogeneización estocástica para tener en cuenta la estructura no homogénea de los policristales. Este modelo brinda una base termodinámica muy buena para simular el comportamiento macroscópico de policristales ferroeléctricos.

Recientemente, Tan y Kochmann (2017) han propuesto un modelo constitutivo para cerámicos ferroeléctricos policristalinos, que vincula los mecanismos de conmutación de dominios y las transiciones de fases en la microescala con la respuesta material electro-termo-mecánicamente acoplada observada a escala macroscópica. Particularmente, han formulado una densidad de energía convexa basada en fracciones de volumen de dominio y extendida a policristales a través de la consideración de Taylor sobre deformaciones uniformes. El marco propuesto es simple y por lo tanto eficiente, manteniendo sin embargo características y mecanismos microestructurales claves al introducir una mezcla de dominios en lugar de utilizar una descripción fenomenológica para la polarización remanente.

A pesar del carácter más general de los modelos micromecánicos, su aplicación a problemas típicos de ingeniería resulta, en general, dificultosa debido a que los modelos son complejos y tienen un gran costo computacional. Es por esta razón que los modelos utilizados en este trabajo para describir a las fases ferroeléctricas son de carácter fenomenológico.

En lo que respecta al modelado de materiales compuestos, Topolov y Bowen (2009) concluyen, luego de realizar un recuento de las diversas estrategias para aproximar la respuesta para distintas configuraciones materiales, que no existe aún una metodología que permita obtener con precisión las propiedades efectivas de materiales compuestos ferroeléctricos. Las estrategias analizadas incluyen métodos de 'promediado directo' de las propiedades de las fases en un volumen macroscópico - analíticamente y mediante el método de los elementos finitos-, métodos basados en 'regularización de la estructura', ecuaciones diferenciales estocásticas o expansio- 
nes viriales, así como el método auto-consistente. Otra estrategia particularmente atractiva para homogeneizar materiales multifase consiste en la construcción de las llamadas 'microgeometrías resolubles'. Por microgeometrías resolubles nos referimos a microgeometrías modelo suficientemente complejas como para reproducir las características geométricas esenciales de las microestructuras reales pero al mismo tiempo suficientemente simples como para permitir el cálculo exacto cuasi analítico de la respuesta macroscópica. La principal ventaja de esta estrategia por sobre los métodos previamente mencionados es que las estimaciones obtenidas satisfacen automáticamente todas las características comúnmente buscadas en una aproximación teórica: estar de acuerdo con restricciones materiales, satisfacer todas las cotas pertinentes, y poseer todas las propiedades de convexidad correspondientes. Esta estrategia fue inicialmente seguida por Maxwell (1873) para estimar las resistencia óhmica total de medios estratificados, y fue desarrollada ampliamente desde entonces para dieléctricos lineales (véase, por ejemplo, Milton, 2002, y sus referencias). En este enfoque resultó fundamental la observación de Maxwell (1873) y Bruggeman (1935) de que el conjunto de microgeometrías resolubles puede agrandarse siguiendo un proceso iterativo en el cual las fases constitutivas de una microgeometría resoluble son, en sí mismas, identificadas con otras microgeometrías resolubles en una escala menor, generándose así microgeometrías jerárquicas de complejidad creciente cuya respuesta macroscópica puede determinarse iterativamente. Por ejemplo, Maxwell (1873) construyó materiales compuestos particulados con respuesta isótropa iterando microgeometrías laminadas anisótropas, en los denominados laminados secuenciales. Bruggeman (1935), por otro lado, construyó materiales compuestos particulados con fracciones volumétricas arbitrarias de partículas iterando microgeometrías con fracciones volumétricas diluidas, lo que se conoce como esquema diferencial. Estos esquemas iterativos ampliaron considerablemente el rango de microestructuras modelables, demostrando ser muy útiles para estimar la respuesta macroscópica de materiales dieléctricos lineales bifásicos.

Ahora bien, todos los modelos mencionados asumen, invariablemente, que la polarización irreversible en el compuesto polarizado es uniforme e igual a la polarización de saturación de la fase ferroeléctrica, despreciando así completamente la compleja variación espacial del campo eléctrico durante el proceso de polarización. Esta hipótesis sigue siendo utilizada incluso en los modelos propuestos más recientemente (por ejemplo, Spinelli y Lopez-Pamies, 2014), a pesar de que es bien sabido que puede resultar particularmente inexacta para sistemas materiales con fuertes contrastes microestructurales tales como los sistemas con partículas metálicas o poros. Más aún, como consecuencia de esta hipótesis todos los modelos mencionados son incapaces de predecir las tensiones residuales provocadas por el proceso de polarización, las cuales 
juegan un rol importante en la fatiga de los materiales ferroeléctricos. En virtud de lo expuesto, el trabajo aquí propuesto tiene como principal objetivo específico derivar una metodología basada en microgeometrías resolubles que incorpore la disipación y el acoplamiento electromecánico y que permita evaluar la precisión de las predicciones basadas en la hipótesis de polarización uniforme.

Cabe señalar también que una segunda fuente de imprecisión en los modelos mencionados proviene de despreciar el hecho de que, lejos de ser dieléctricos perfectamente aislantes, muchos de los materiales ferroeléctricos son en realidad semiconductores de banda prohibida ancha, por lo que la polarización intrínseca puede verse mejorada por medio de una polarización extrínseca producida por las cargas espaciales en el entorno de interfases microestructurales que obstruyen la migración de iones. Existen diversos estudios que introducen la influencia de la conductividad y la presencia de cargas libres en el material, por ejemplo, introduciendo la densidad de carga en el material como una variable del problema (Schwaab et al., 2013; Xiao y Bhattacharya, 2008) o considerando la conductividad en el borde de grano (Wang et al., 2014). Los estudios que intentan correlacionar este comportamiento dieléctrico con la morfología microestructural son limitados y casi invariablemente se restringen a las microestructuras y las leyes constitutivas locales más simples (por ejemplo, Lopez-Pamies et al., 2014; Sørensen, 1997; Tonkoshkur et al., 2017; Trukhan, 1963). Una excepción a esto es el trabajo reciente de Lefèvre y Lopez-Pamies (2017), en donde se hace uso de expansiones asintóticas de dos escalas para derivar una respuesta electromecánica homogeneizada bajo hipótesis bastante amplias, aunque asume una clase especial de densidades de cargas espaciales elegidas sobre la base de la conveniencia matemática en lugar de las premisas establecidas por la electroquímica para especies cargadas en sólidos. El trabajo aquí propuesto tiene como segundo objetivo específico incorporar el efecto de cargas libres en la descripción de la respuesta de materiales dieléctricos con segundas fases.

\subsection{Descripción del trabajo}

En este trabajo se estudia el comportamiento electromecánico de materiales compuestos electroactivos mediante la teoría electromecánica de medios continuos (véase, por ejemplo, Gurtin et al., 2010) y la teoría matemática de homogeneización (véase, por ejemplo, Milton, 2002) utilizando microgeometrías resolubles. La metodología propuesta consiste en idealizar al material compuesto como un medio continuo con microestructuras a diferentes escalas y relacionar su comportamiento macroscópico con el comportamiento de las distintas fases constitutivas y la morfología microestructural. Se asume que las deformaciones son pequeñas y que los materiales electroactivos disipan energía tanto eléctrica como mecánica. El trabajo se apoya 
en avances recientes en la teoría electromecánica de medios continuos disipativos y métodos de homogeneización.

En el capítulo 2 se presenta una teoría electromecánica para medios continuos heterogéneos deformables. La misma se basa en una formulación recientemente propuesta por Miehe y Rosato (2011) para sólidos ferroeléctricos disipativos. En ella se caracteriza el estado del material por un tensor de deformación infinitesimal, un vector de polarización eléctrica y un vector de polarización permanente que obedecen leyes constitutivas termodinámicamente consistentes derivadas de una energía de Helmholtz y un potencial de disipación. Luego, el problema electromecánico se discretiza en el tiempo siguiendo un esquema de tipo Euler implícito y se escribe un principio variacional incremental con variables internas que arroja como resultado los campos electromecánicos en un instante dado en función de los valores de dichos campos en el instante anterior. En el trabajo de Miehe y Rosato (2011) se demuestra que la respuesta electromecánica típica de materiales ferroeléctricos - como la mostrada en la figura 1.6a- puede representarse mediante potenciales de disipación del tipo ley de la potencia. El problema matemático planteado es pues muy similar al de la elasto-viscoplasticidad, lo cual permitirá utilizar técnicas de resolución ya desarrolladas en ese contexto. Predicciones obtenidas para materiales homogéneos con esta teoría de medios continuos se presentan en el capítulo 3, en donde además se extiende el uso de esta teoría desarrollada en el contexto de materiales ferroeléctricos a materiales ferromagnéticos y se discuten posibles limitaciones para su aplicabilidad en los diversos sistemas materiales.

Una vez formulada la teoría de medios continuos a nivel micrométrico, en el capítulo 4 se procede a homogeneizar las ecuaciones resultantes para obtener el comportamiento macroscópico del material electroactivo en términos del comportamiento local y de la microestructura. La homogeneización es una técnica matemática que permite evaluar la solución oscilatoria de un sistema de ecuaciones diferenciales con coeficientes periódicos cuando el período de los coeficientes tiende a cero. En el caso de un material compuesto, el período de los coeficientes está asociado con la relación entre la longitud característica de las heterogeneidades — por ejemplo, diámetro de partículas- y la longitud característica del espécimen. Para resolver luego el problema homogeneizado se propone aquí generalizar la estrategia basada en 'microgeometrías resolubles' de Idiart (2014) para materiales dieléctricos no disipativos al caso de materiales dieléctricos con respuestas hereditarias. Esta estrategia aprovecha la solución cuasi analítica de laminados simples, con la cual es posible obtener predicciones para materiales compuestos representativos en el capítulo 5. Se presentan estimaciones para la respuesta de materiales ferroeléctricos con inclusiones metálicas, ferromagnéticas y poros y se discute sobre la validez de las predicciones obtenidas. 
Por otro lado, en el capítulo 6 se encara la problemática de materiales dieléctricos rígidos con presencia de cargas libres, la que se incorpora en la teoría siguiendo los lineamientos propuestos por Maugin y Daher (1986) y Xiao y Bhattacharya (2008) en el contexto de semiconductores elásticos. Dada la complejidad de la problemática, se procede aquí acotando el problema de estudio y focalizando la atención en la predicción del comportamiento de materiales dieléctricos rígidos considerando el efecto de las cargas libres y sin tener en cuenta fenómenos ferroeléctricos. Predicciones para sistemas materiales sencillos se presentan en el capítulo 7, con el foco puesto en lograr una mejor comprensión del efecto de los iones móviles en el comportamiento del material y, si bien no se consideran aquí las características ferroeléctricas de los materiales, se pretende sentar las bases para poder considerar el carácter semiconductor en futuros trabajos.

Finalmente, en el capítulo 8 se resumen los principales resultados obtenidos y se presenta una discusión con las conclusiones, recomendaciones y trabajo futuro relacionado con el tema de estudio.

\subsection{Publicaciones derivadas de la tesis}

\section{Publicaciones científicas}

- Idiart M.I., Bottero C.J.: "Space-charge polarization by confined ion migration in microstructured solid dielectrics", Journal of the Mechanics and Physics of Solids, enviado.

- Bottero C.J., Idiart M.I.: "An evaluation of a class of phenomenological theories of ferroelectricity and ferromagnetism". Journal of Engineering Mathematics, enviado.

- Bottero C.J., Idiart M.I.: "Influence of second-phase inclusions on the electrodeformation of ferroelectric ceramics", International Journal of Solids and Structures 80, 2016, pp. 381-392, ISSN 0020-7683.

- Bottero C.J., Idiart M.I.: "Propiedades piezoeléctricas de ferrocerámicos microestructurados". Tercer Seminario de Ecamat Fenómenos de transporte en sólidos deformables: teorías de homogenización; compilado por Mauricio Chocrón, Cecilia Conti. Ciudad Autónoma de Buenos Aires, Academia Nacional de Ciencias de Buenos Aires, 2016, pp. 19-34, ISBN 978-987-537-136-1.

- Bottero C.J., Idiart M.I.: "Influence of metallic inclusions on the electromechanical response of ferroelectric ceramics". Actas del III Congreso Argentino de Ingeniería Aeronáutica, 1a ed.Universidad Nacional de La Plata, 2014. EBook. ISBN 978950-34-1152-0 


\section{Presentaciones en Congresos y Jornadas Científicas}

- Idiart M.I., Bottero C.J.: "Static space-charge polarization in microstructured solid dielectrics". 10 ${ }^{\text {th }}$ European Solid Mechanics Conference, Bologna, Italia, 2-6 julio 2018.

- Idiart M.I., Bottero C.J.: "Space-charge polarization in microstructured solid dielectrics". Workshop on the Multiphysics Behavior of Rubber, Marsella, Francia, 30 noviembre - 1 diciembre 2017.

- Idiart M.I., Bottero C.J.: "Space-charge polarization in microstructured solid dielectrics". XXIII Congreso sobre Métodos Numéricos y sus Aplicaciones, La Plata, 7-10 noviembre 2017.

- Bottero C.J., Idiart M.I.: "Piezoelectric response and homogenization estimates in two-phase ferroelectric composites with thermal stresses and free charges effects". XXII Congreso sobre Métodos Numéricos y sus Aplicaciones, Córdoba, 8-11 noviembre 2016.

- Bottero C.J., Idiart M.I.: "Influencia de partículas de segunda fase en la electrodeformabilidad de cerámicos ferroeléctricos". VI Workshop de Modelado multiescala, Universidad Nacional de Rosario. Rosario, 28-29 agosto 2015.

- Bottero C.J., Idiart M.I.: "Influence of metallic inclusions on the electromechanical response of ferroelectric ceramics". III Congreso Argentino de Ingeniería Aeronáutica, La Plata, 12-14 noviembre 2014.

- Bottero C.J., Idiart M.I.: "Estimación de la respuesta electromecánica de materiales compuestos ferroeléctricos bifásicos". I Jornadas de Tesistas. Facultad de Ingeniería, Universidad Nacional de La Plata, 9 octubre 2014.

- Bottero C.J., Idiart M.I.: "Homogenization estimates for the electromechanical response of two-phase ferroelectric composites". XXI Congreso sobre Métodos Numéricos y sus Aplicaciones, San Carlos de Bariloche, 23-26 septiembre 2014.

- Bottero C.J., Idiart M.I.: "Respuesta disipativa de materiales compuestos ferroeléctricos". Póster. VI Latin American Symposium on High Performance Computing HPCLatAm 2013, Mendoza, Argentina, 22-30 julio 2013. 


\section{Teoría de medios continuos para el comportamiento electromecánico de ferrocerámicos}

\subsection{Introducción}

En este capítulo se presenta una clase de teorías multiaxiales para sólidos ferróicos policristalinos elásticos basada en la descomposición aditiva de la deformación y de la densidad de dipolos eléctricos o magnéticos en variables internas reversibles asociadas a la elasticidad y la perturbación de los dipolos, y en variables internas irreversibles asociadas con las alteraciones de los dipolos o conmutación de dominios (Kamlah, 2001; McMeeking y Landis, 2002; Miehe et al., 2011a; Miehe y Rosato, 2011). En la misma, el comportamiento electrodeformable de las fases constitutivas se describe por medio de una densidad de energía almacenada y un potencial de disipación de acuerdo a la teoría de materiales estándar generalizados. Estas teorías son capaces de reproducir características esenciales de la respuesta de materiales ferróicos, como ser curvas de tensión-deformación no lineales, curvas de ciclos de histéresis y rotación de dipolos, y son adecuadas para su implementación numérica en códigos de elementos finitos relativamente sencillos. Códigos de este tipo han sido utilizados para simular procesos electro-magneto-mecánicos que incluyen la iniciación y propagación de fisuras en ferrocerámicos monolíticos (Linder y Miehe, 2012), la polarización de materiales ferróicos multifase (Kamlah y Böhle, 2001; Labusch et al., 2014; Zhang et al., 2005) y el acoplamiento en materiales compuestos multiferróicos (Miehe et al., 2011b).

\subsection{Marco teórico}

\subsubsection{Sistema material}

La clase de teorías fenomenológicas consideradas en este trabajo se basan en realizar una descomposición aditiva de la deformación infinitesimal y de la densidad de dipolos eléctricos en variables internas reversibles asociadas a alteraciones de los dipolos o conmutación de dominios. Este tipo de teorías son relevantes para sólidos policristalinos ferróicos sometidos a estados de carga electromecánicos cuasi estáticos. Se centra la atención en una subclase de teorías simples propuestas por McMeeking y Landis (2002) y refinadas por Miehe y Rosato (2011). En las mismas 


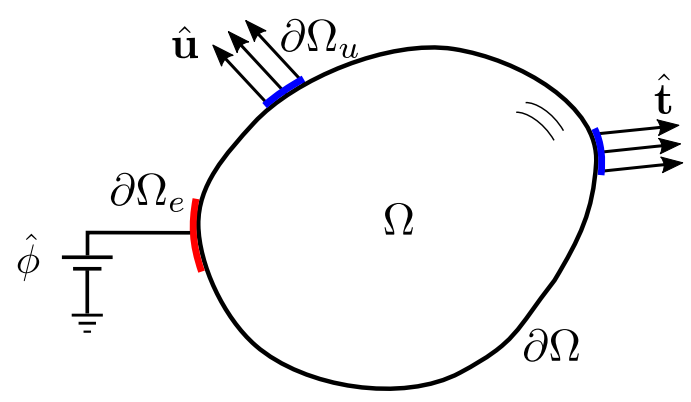

Figura 2.1: Dominio y condiciones de borde para un ferrocerámico

se describe el estado electromecánico del sólido a través de un tensor deformación $\varepsilon$, un vector polarización $\mathbf{P}$, y un vector de polarización irreversible p que caracteriza la magnitud y orientación promedio de los dipolos eléctricos permanentes relativos a la configuración libre de tensiones y sin polarización neta. El hecho de que los dipolos permanentes se caractericen por una única variable simplifica la formulación matemática considerablemente pero hace que la teoría sea incapaz de tener en cuenta la conmutación de dominios inducida por estados de carga puramente mecánicos. Es de esperar entonces que las predicciones sean razonablemente exactas cuando los campos eléctricos sean altos, pero las tensiones sean bajas. A pesar de esto, las predicciones obtenidas deberían ser matemáticamente estables en todo el rango de intensidades de campo eléctrico y de tensiones físicamente admisibles.

El sistema material bajo estudio se idealiza como un cuerpo homogéneo que ocupa un dominio $\Omega$ compuesto por un material ferrocerámico, como se presenta en la figura 2.1. Se consideran únicamente procesos isotérmicos producidos por interacciones electromecánicas cuasi estáticas. Estas interacciones son ejercidas por un potencial eléctrico fijo $\hat{\phi}_{e}$ aplicado mediante electrodos superficiales ocupando una porción $\partial \Omega_{e}$ del borde del espécimen $\partial \Omega$, por un desplazamiento de la superficie $\hat{\mathbf{u}}$ aplicado en una porción $\partial \Omega_{u}$ del borde del cuerpo, y por una tracción $\hat{\mathbf{t}}$ aplicada en el complemento del borde del cuerpo.

\subsubsection{Ecuaciones de campo}

La densidad volumétrica de carga eléctrica del dominio $\Omega$ solamente considera cargas debido a la polarización, definiéndose como

$$
\rho_{c}=-\nabla \cdot \mathbf{P},
$$


mientras que la densidad superficial de carga eléctrica en el borde exterior se considera como

$$
\sigma_{c}(\mathbf{x})= \begin{cases}-\llbracket \mathbf{P} \rrbracket \cdot \mathbf{n} & \text { en } \quad \partial \Omega \backslash \partial \Omega_{e} \\ -\llbracket \mathbf{P} \rrbracket \cdot \mathbf{n}+\sigma_{f}(\mathbf{x}) & \text { en } \quad \partial \Omega_{e},\end{cases}
$$

donde $\sigma_{f}(\mathbf{x})$ es una densidad superficial de cargas y $\llbracket \cdot \rrbracket$ representa el salto a través del borde del dominio con vector normal saliente $\mathbf{n}$.

Las ecuaciones que gobiernan el problema son entonces — véase, por ejemplo, Kamlah $(2001)$

$$
\begin{array}{llll}
\nabla \cdot \mathbf{D}=0 & \text { y } & \mathbf{E}=-\nabla \phi & \text { en } \mathbb{R}^{3} \backslash \partial \Omega \\
\nabla \cdot \boldsymbol{\sigma}=\mathbf{0} & \text { y } & \boldsymbol{\varepsilon}=\nabla \otimes_{s} \mathbf{u} & \text { en } \Omega
\end{array}
$$

con

$$
\mathbf{D}= \begin{cases}\epsilon_{0} \mathbf{E} & \text { en } \mathbb{R}^{3} \backslash \Omega \\ \epsilon_{0} \mathbf{E}+\mathbf{P} & \text { en } \Omega\end{cases}
$$

y las condiciones de borde

$$
\begin{array}{lllll}
\phi=\hat{\phi} & \text { en } \partial \Omega_{e} & \text { y } & \llbracket \mathbf{D} \rrbracket \cdot \mathbf{n}=0 & \text { en } \partial \Omega \backslash \partial \Omega_{e} \\
\mathbf{u}=\hat{\mathbf{u}} & \text { en } \partial \Omega_{u} & \text { y } & \boldsymbol{\sigma} \mathbf{n}=\hat{\mathbf{t}} & \text { en } \partial \Omega \backslash \partial \Omega_{u}
\end{array}
$$

En estas expresiones, $\phi$ y $\mathbf{u}$ son campos continuos que representan el potencial electrostático y el desplazamiento, $\mathbf{D}, \mathbf{E}, \mathbf{P}, \boldsymbol{\sigma}$ y $\boldsymbol{\varepsilon}$ son, respectivamente, el desplazamiento eléctrico, la intensidad de campo eléctrico, la polarizabilidad del material y los tensores de tensión y deformación, $\llbracket \cdot \rrbracket$ denota el salto a través de $\partial \Omega, \mathbf{n}$ es el vector normal hacia afuera de $\partial \Omega$, y $\epsilon_{0}$ es la permitividad eléctrica del vacío. A su vez, $\nabla$ es el operador nabla estándar y el símbolo $\otimes_{s}$ representa la parte simétrica del producto tensorial. El potencial electrostático debe además anularse en el infinito, es decir, $\phi \rightarrow 0$ cuando $|\mathbf{x}| \rightarrow \infty$.

\subsubsection{Termodinámica y relaciones constitutivas}

Las ecuaciones de campo anteriores deben ser complementadas con relaciones constitutivas que describan la respuesta electromecánica del material. Se adopta aquí el enfoque de Bassiouny et al. (1988) en el cual los procesos disipativos se caracterizan mediante una polarización irreversible p que juega el rol de una variable interna. Este marco teórico es lo suficientemente general para caracterizar respuestas simples como ser la polarizabilidad lineal, así como respuestas complejas como la ferroelectricidad 
dependiente de la tasa de carga — véase, por ejemplo, Kamlah (2001), Miehe y Rosato (2011).

La energía total del sistema material y el campo eléctrico puede escribirse como

$$
\mathfrak{E}=\int_{\Omega} \mathfrak{e}(\varepsilon, \mathbf{P}, \mathrm{p}) \mathrm{d} V+\int_{\mathbb{R}^{3}} \frac{1}{2} \epsilon_{0} \mathbf{E}^{2} \mathrm{~d} V
$$

donde el primer término representa, a partir de la energía libre de Helmholtz $\mathfrak{e}$, la energía almacenada en el sistema material y caracteriza completamente su respuesta electromecánica, mientras que el segundo término es la energía electrostática del campo eléctrico. A su vez, la disipación del sistema se asume de la forma

$$
\mathcal{D}=\int_{\Omega} \frac{\partial \varphi}{\partial \dot{\mathrm{p}}}(\dot{\mathrm{p}}) \cdot \dot{\mathrm{p}} \mathrm{d} V
$$

donde el potencial de disipación $\varphi$ es una función positiva convexa de la tasa de polarización irreversible $\dot{\mathrm{p}}$ tal que $\varphi(\mathbf{0})=0$, que se utiliza para caracterizar la conmutación de dominios microscópicos en la fase ferroeléctrica. La forma (2.9) garantiza la disipación positiva requerida por los principios de la termodinámica.

Los argumentos termodinámicos implican entonces que un conjunto amplio de relaciones constitutivas del material están dadas por (véase, por ejemplo, Bassiouny et al., 1988)

$$
\mathbf{E}=\frac{\partial \mathfrak{e}}{\partial \mathbf{P}}(\varepsilon, \mathbf{P}, \mathrm{p}), \quad \boldsymbol{\sigma}=\frac{\partial \mathfrak{e}}{\partial \boldsymbol{\varepsilon}}(\varepsilon, \mathbf{P}, \mathrm{p}) \quad \mathrm{y} \quad \frac{\partial \mathfrak{e}}{\partial \mathrm{p}}(\varepsilon, \mathbf{P}, \mathrm{p})+\frac{\partial \varphi}{\partial \dot{\mathrm{p}}}(\dot{\mathrm{p}})=\mathbf{0},
$$

donde las primeras dos expresiones relacionan la intensidad de campo eléctrico $\mathbf{E}$ y el tensor de tensiones $\boldsymbol{\sigma}$ con la polarización y la deformación, y la última expresión provee la ley de evolución para la polarización irreversible p.

Para el caso de potenciales no suaves, las derivadas en (2.10) deben entenderse en el sentido del subdiferencial del análisis convexo. Estas relaciones constitutivas se ajustan al denominado modelo de material estándar generalizado siempre y cuando la energía e sea convexa (Germain et al., 1983). En ese caso, la polarización puede ser eliminada de la descripción constitutiva en favor de la intensidad de campo eléctrico definiendo la densidad de energía complementaria

$$
\psi(\boldsymbol{\sigma}, \mathbf{E}, \mathrm{p}) \doteq \sup _{\mathbf{P}, \boldsymbol{\varepsilon}}[\boldsymbol{\sigma} \cdot \boldsymbol{\varepsilon}+\mathbf{E} \cdot \mathbf{P}-\mathfrak{e}(\varepsilon, \mathbf{P}, \mathrm{p})]+\frac{1}{2} \epsilon_{0} \mathbf{E}^{2}
$$

donde el primer término corresponde a la transformada parcial de Legendre de $\mathfrak{e}$ con respecto a $\mathbf{P}$ y $\boldsymbol{\varepsilon}$. Cabe destacar que la función $\psi$ es por lo tanto convexa en $\mathbf{E}$ y $\boldsymbol{\sigma}$ pero cóncava en p. Las relaciones constitutivas (2.10) pueden entonces reescribirse 
como

$$
\mathbf{D}=\frac{\partial \psi}{\partial \mathbf{E}}(\boldsymbol{\sigma}, \mathbf{E}, \mathrm{p}), \quad \boldsymbol{\varepsilon}=\frac{\partial \psi}{\partial \boldsymbol{\sigma}}(\boldsymbol{\sigma}, \mathbf{E}, \mathrm{p}) \quad \text { y } \quad \frac{\partial \psi}{\partial \mathrm{p}}(\boldsymbol{\sigma}, \mathbf{E}, \mathrm{p})-\frac{\partial \varphi}{\partial \dot{\mathrm{p}}}(\dot{\mathrm{p}})=\mathbf{0} .
$$

Las ecuaciones de campo y las condiciones de borde (2.3)-(2.7), junto con las relaciones constitutivas (2.12) y condiciones iniciales apropiadas, definen completamente la respuesta electromecánica del material estudiado.

\subsubsection{Relaciones constitutivas incrementales}

Siguiendo al trabajo de Miehe (2002), las ecuaciones (2.3)-(2.12) definidas en el dominio del material pueden discretizarse en el tiempo $\left(t_{0}=0, t_{1}, \ldots, t_{i}, t_{i+1}, \ldots, t_{N}=\right.$ $T$ ) siguiendo un esquema de Euler implícito, de modo que los campos $\phi_{i+1}$ y $p_{i+1}$ en el tiempo $t_{i+1}$ son solución de

$$
\begin{aligned}
& \mathbf{E}_{i+1}=-\nabla \phi_{i+1}, \quad \nabla \cdot \mathbf{D}_{i+1}=0 \quad \text { en } \quad \Omega, \\
& \nabla \cdot \boldsymbol{\sigma}_{i+1}=\mathbf{0}, \quad \boldsymbol{\varepsilon}_{i+1}=\nabla \otimes_{s} \mathbf{u}_{i+1} \quad \text { en } \quad \Omega, \\
& \mathbf{D}_{i+1}=\frac{\partial \psi}{\partial \mathbf{E}}\left(\boldsymbol{\sigma}_{i+1}, \mathbf{E}_{i+1}, \mathrm{p}_{i+1}\right), \quad \boldsymbol{\varepsilon}_{i+1}=\frac{\partial \psi}{\partial \boldsymbol{\sigma}}\left(\boldsymbol{\sigma}_{i+1}, \mathbf{E}_{i+1}, \mathrm{p}_{i+1}\right) \quad \text { en } \quad \Omega, \\
& \phi_{i+1}=\hat{\phi}_{i+1} \quad \text { en } \partial \Omega_{e} \quad \text { y } \quad \llbracket \mathbf{D}_{i+1} \rrbracket \cdot \mathbf{n}=0 \quad \text { en } \partial \Omega \backslash \partial \Omega_{e}, \\
& \mathbf{u}_{i+1}=\hat{\mathbf{u}}_{i+1} \quad \text { en } \partial \Omega_{u} \quad \text { y } \quad \boldsymbol{\sigma}_{i+1} \mathbf{n}=\hat{\mathbf{t}}_{i+1} \quad \text { en } \partial \Omega \backslash \partial \Omega_{u}, \\
& \frac{\partial \psi}{\partial \mathrm{p}}\left(\boldsymbol{\sigma}_{i+1}, \mathbf{E}_{i+1}, \mathrm{p}_{i+1}\right)-\frac{\partial \varphi}{\partial \dot{p}}\left(\frac{\mathrm{p}_{i+1}-\mathrm{p}_{i}}{\Delta t}\right)=\mathbf{0} \quad \text { en } \quad \Omega,
\end{aligned}
$$

donde $\phi_{i} \mathrm{y} \mathrm{p}_{i}$ son los valores conocidos de los campos en instante de tiempo previo $t_{i}$ y $\Delta t=t_{i+1}-t_{i}$ es el paso de tiempo, que se asume como fijo - es decir, independiente de $i$ - por simplicidad. La observación clave en el contexto de estas ecuaciones es que las relaciones constitutivas (2.15) pueden escribirse como

$$
\mathbf{D}_{i+1}=\frac{\partial w}{\partial \mathbf{E}}\left(\boldsymbol{\sigma}_{i+1}, \mathbf{E}_{i+1} ; p_{i}\right) \quad \text { y } \quad \boldsymbol{\varepsilon}_{i+1}=\frac{\partial w}{\partial \boldsymbol{\sigma}}\left(\boldsymbol{\sigma}_{i+1}, \mathbf{E}_{i+1} ; \mathrm{p}_{i}\right),
$$

donde $w$ es un potencial incremental definido por

$$
w\left(\boldsymbol{\sigma}, \mathbf{E} ; \mathrm{p}_{i}\right)=\sup _{\mathrm{p}} J\left(\boldsymbol{\sigma}, \mathbf{E}, \mathrm{p} ; \mathrm{p}_{i}\right)
$$

con

$$
J\left(\boldsymbol{\sigma}, \mathbf{E}, \mathrm{p} ; \mathrm{p}_{i}\right)=\psi(\boldsymbol{\sigma}, \mathbf{E}, \mathrm{p})-\Delta t \varphi\left(\frac{\mathrm{p}-\mathrm{p}_{i}}{\Delta t}\right),
$$


donde la solución óptima p corresponde a la polarización irreversible $\mathrm{p}_{i+1}$ en el paso de tiempo $i+1$ que es solución de la ley de evolución discretizada (2.18) para el valor de $\mathrm{p}_{i}$ dado.

Esta observación surge del hecho que $\psi$ es cóncavo en p y $\varphi$ es convexo en $\dot{p}$, y que las ecuaciones de Euler-Lagrange asociadas con el supremo en (2.20) para $\mathbf{E}_{i+1} \mathrm{y}$ $\boldsymbol{\sigma}_{i+1}$ son precisamente las relaciones $(2.18)$ para $\mathrm{p}_{i+1}$. Además, que las derivadas de $w$ con respecto al campo eléctrico y al campo de tensiones permiten obtener el desplazamiento eléctrico $\mathbf{D}_{n+1}$ y la deformación $\boldsymbol{\varepsilon}_{n+1}$, respectivamente, surge de (2.15) y de la estacionaridad de (2.20) con respecto a p. Se puede demostrar fácilmente que el potencial incremental $w$ es una función convexa de $\mathbf{E}$ y $\boldsymbol{\sigma}$.

Teniendo en cuenta (2.19), las ecuaciones (2.13)-(2.17) adoptan la forma

$$
\begin{aligned}
& \mathbf{E}=-\nabla \phi, \quad \nabla \cdot \mathbf{D}=0 \quad \text { en } \quad \Omega, \\
& \nabla \boldsymbol{\sigma}=\mathbf{0}, \quad \boldsymbol{\varepsilon}=\nabla \otimes_{s} \mathbf{u} \quad \text { en } \quad \Omega, \\
& \mathbf{D}=\frac{\partial w}{\partial \mathbf{E}}\left(\boldsymbol{\sigma}, \mathbf{E} ; \mathrm{p}_{i}\right), \quad \boldsymbol{\varepsilon}=\frac{\partial w}{\partial \boldsymbol{\sigma}}\left(\boldsymbol{\sigma}, \mathbf{E} ; \mathrm{p}_{i}\right) \quad \text { en } \quad \Omega, \\
& \phi=\hat{\phi} \quad \text { en } \partial \Omega_{e} \quad \text { y } \quad \llbracket \mathbf{D} \rrbracket \cdot \mathbf{n}=0 \quad \text { en } \partial \Omega \backslash \partial \Omega_{e}, \\
& \mathbf{u}=\hat{\mathbf{u}} \quad \text { en } \partial \Omega_{u} \quad \text { y } \quad \boldsymbol{\sigma} \mathbf{n}=\hat{\mathbf{t}} \quad \text { en } \partial \Omega \backslash \partial \Omega_{u},
\end{aligned}
$$

donde el subíndice $i+1$ ha sido eliminado para facilitar la notación. Para $\mathrm{p}_{i}$ dado y prescribiendo $\mathbf{E}$ y $\boldsymbol{\sigma}$, estas ecuaciones proveen los campos solución para el tiempo $t_{i+1}$.

\subsubsection{Formas específicas de las funciones termodinámicas}

A pesar de que el enfoque utilizado en este trabajo permite formas generales para la energía e y para el potencial de disipación $\varphi$, resulta útil dejar constancia aquí de algunas formas específicas de uso común y que serán empleadas en cálculos posteriores.

i) Muchos sólidos no polares pueden ser caracterizados como dieléctricos isótropos ideales con una respuesta lineal no disipativa, en cuyo caso los potenciales toman la forma

$$
\mathfrak{e}(\varepsilon, \mathbf{P}, \mathrm{p})=\frac{1}{2} \varepsilon \cdot \mathbb{C} \boldsymbol{\varepsilon}+\frac{1}{2} \mathbf{P} \cdot \boldsymbol{\kappa} \mathbf{P} \quad \text { y } \quad \varphi(\dot{\mathrm{p}})= \begin{cases}0 & \dot{\mathrm{p}}=0 \\ +\infty & \text { c.c. }\end{cases}
$$

donde los tensores elasticidad $\mathbb{C}$ y polarizabilidad eléctrica $\boldsymbol{\kappa}$ están dados en términos 
de las constantes isótropas por

$$
\mathbb{C}=\lambda \mathbf{I} \otimes \mathbf{I}+2 \mu \mathbf{I} \quad \text { y } \quad \boldsymbol{\kappa}=\kappa \mathbf{I} .
$$

En estas expresiones, I y II son los tensores identidad segundo y cuarto orden con simetría mayor y menor, y $\kappa, \lambda$ y $\mu$ representan, respectivamente, la polarizabilidad y las constantes de Lamé del sólido.

La densidad de energía complementaria correspondiente está dada por

$$
\psi(\boldsymbol{\sigma}, \mathbf{E}, \mathrm{p})=\frac{1}{2} \boldsymbol{\sigma} \cdot \mathbb{S} \boldsymbol{\sigma}+\frac{1}{2} \mathbf{E} \cdot \boldsymbol{\epsilon} \mathbf{E},
$$

donde $\boldsymbol{\epsilon}=\epsilon_{0} \mathbf{I}+\boldsymbol{\kappa}^{-1}=\epsilon \mathbf{I}$ y $\mathbb{S}=\mathbb{C}^{-1}$ representan, respectivamente, los tensores permitividad y flexibilidad del sólido. Las relaciones constitutivas correspondientes están dadas por

$$
\boldsymbol{\varepsilon}=\mathbb{S} \boldsymbol{\sigma} \quad \mathrm{y} \quad \mathbf{D}=\epsilon \mathbf{E} .
$$

Un conductor perfecto en el cual el campo eléctrico debe ser nulo puede caracterizarse como un material con $\kappa \rightarrow 0(\epsilon \rightarrow \infty)$.

ii) Los sólidos policristalinos con ferroelectricidad isótropa son comúnmente caracterizados con potenciales de la forma - véase, por ejemplo, Kamlah (2001), McMeeking y Landis (2002), Miehe y Rosato (2011) -

$$
\begin{array}{r}
\mathfrak{e}(\boldsymbol{\varepsilon}, \mathbf{P}, \mathrm{p})=\frac{1}{2}(\boldsymbol{\varepsilon}-\hat{\boldsymbol{\varepsilon}}(\mathrm{p})) \cdot \mathbb{C}(\boldsymbol{\varepsilon}-\hat{\boldsymbol{\varepsilon}}(\mathrm{p}))+\frac{1}{2}(\mathbf{P}-\mathrm{p}) \cdot \boldsymbol{\kappa}(\mathbf{P}-\mathrm{p})+ \\
(\boldsymbol{\varepsilon}-\hat{\boldsymbol{\varepsilon}}(\mathrm{p})) \cdot \mathbf{h}(\mathrm{p})(\mathbf{P}-\mathrm{p})+\mathfrak{e}_{p d}(\mathrm{p}),
\end{array}
$$

donde los tensores elasticidad $\mathbb{C}$ y polarizabilidad eléctrica $\boldsymbol{\kappa}$ están dados en términos de las constantes isótropas por (2.28) y el tensor piezoeléctrico está dado en términos de tres módulos piezoeléctricos por

$$
\mathbf{h}(\mathrm{p})=\frac{|\mathrm{p}|}{p_{s}}\left(\alpha_{0} \frac{\mathrm{p}}{|\mathrm{p}|} \otimes \frac{\mathrm{p}}{|\mathrm{p}|} \otimes \frac{\mathrm{p}}{|\mathrm{p}|}+\alpha_{\perp} \mathbf{I} \otimes \frac{\mathrm{p}}{|\mathrm{p}|}+\alpha_{=} \frac{\mathrm{p}}{|\mathrm{p}|} \otimes_{s} \mathbf{I}\right)
$$

En estas expresiones el símbolo $\otimes_{s}$ representa la simetrización con respecto a los primeros dos índices, y $\alpha_{0}, \alpha_{\perp}$ y $\alpha_{=}$son, respectivamente, los módulos piezoeléctricos axial, lateral y de distorsión.

En este modelo se asume que la polarización $\mathbf{P}$ y la deformación $\varepsilon$ son composiciones aditivas de una parte reversible $(\mathbf{P}-$ p) y $(\varepsilon-\hat{\varepsilon})$, y una parte irreversible p y $\hat{\varepsilon}$.

Los primeros tres términos de (2.31) representan las energías elástica, de polarización 
eléctrica y piezoeléctrica, respectivamente, y definen una forma cuadrática que debe satisfacer las desigualdades

$$
\frac{\alpha_{=}^{2}}{4 \mu \kappa}<1 \quad \text { y } \quad \frac{\mu}{3 \lambda+2 \mu} \frac{\left(\alpha_{0}+\alpha_{=}\right)^{2}+\left(\alpha_{=}+\alpha_{\perp}\right)^{2}+6 \alpha_{0} \alpha_{\perp}-\alpha_{=}^{2}}{3 \mu \kappa-\left(\alpha_{0}+\alpha_{=}\right)^{2}}<1
$$

para cumplir con el requisito de convexidad en la energía libre de Helmholtz. A su vez, la energía almacenada $\mathfrak{e}_{p d}$ debido a los dipolos permanentes se define como

$$
\mathfrak{e}_{p d}(\mathrm{p})=-h_{s} p_{s}^{2}\left[\ln \left(1-\frac{|\mathrm{p}|}{p_{s}}\right)+\frac{|\mathrm{p}|}{p_{s}}\right]
$$

donde $p_{s}$ es la polarización de saturación y $h_{s}$ es un parámetro material que caracteriza la pendiente de la histéresis eléctrica, mientras que la deformación asociada con los dipolos permanentes está dada por

$$
\hat{\boldsymbol{\varepsilon}}(\mathrm{p})=\frac{3}{2} \varepsilon_{s}\left(\frac{|\mathrm{p}|}{p_{s}}\right)^{\gamma} \frac{\mathrm{p}}{|\mathrm{p}|} \otimes_{d} \frac{\mathrm{p}}{|\mathrm{p}|}
$$

donde el símbolo $\otimes_{d}$ denota la parte desviadora del producto tensorial, $\varepsilon_{s}$ es la deformación uniaxial correspondiente con saturación de la polarización, y el exponente $\gamma$ es un parámetro material adicional que caracteriza la forma del ciclo de histéresis de modo tal que $\gamma \geq 1$. Finalmente, las teorías postulan un potencial de disipación de la forma

$$
\varphi(\dot{\mathrm{p}})=e_{c}|\dot{\mathrm{p}}|+\frac{e_{0} \dot{p}_{0}}{1+n}\left(\frac{|\dot{\mathrm{p}}|}{\dot{p}_{0}}\right)^{1+n}
$$

donde $e_{c}$ es la intensidad del campo coercitivo del sólido - es decir, el nivel de campo eléctrico por encima del cual la conmutación de dipolos comienza-, $e_{0}$ y $\dot{p}_{0}$ son, respectivamente, un campo eléctrico de referencia y una tasa de polarización que caracterizan la dependencia con la tasa del proceso de conmutación, y $n$ es un exponente que caracteriza la sensibilidad a la tasa, tal que $0 \leq n \leq 1$.

Finalmente, la densidad de energía complementaria está dada por

$$
\begin{array}{r}
\psi(\boldsymbol{\sigma}, \mathbf{E}, \mathrm{p})=\frac{1}{2} \boldsymbol{\sigma} \cdot \hat{\mathrm{S}}(\mathrm{p}) \boldsymbol{\sigma}+\frac{1}{2} \mathbf{E} \cdot \hat{\boldsymbol{\epsilon}}(\mathrm{p}) \mathbf{E}-\boldsymbol{\sigma} \cdot \hat{\mathbf{h}}(\mathrm{p}) \mathbf{E}+\boldsymbol{\sigma} \cdot \hat{\boldsymbol{\varepsilon}}(\mathrm{p})+ \\
+\mathbf{E} \cdot \mathrm{p}-\mathfrak{e}_{p d}(\mathrm{p}),
\end{array}
$$


con

$$
\begin{aligned}
\hat{\mathbb{S}}(\mathrm{p}) & =\left(\mathbb{C}-\mathbf{h} \boldsymbol{\kappa}^{-1} \mathbf{h}^{T}\right)^{-1}, \\
\hat{\boldsymbol{\epsilon}}(\mathrm{p}) & =\hat{\boldsymbol{\kappa}}^{-1}+\epsilon_{0} \mathbf{I}, \\
\hat{\mathbf{h}}(\mathrm{p}) & =\frac{1}{2}\left(\hat{\mathrm{S}} \mathbf{h} \boldsymbol{\kappa}^{-1}+\mathbf{S} \mathbf{h} \hat{\boldsymbol{\kappa}}^{-1}\right),
\end{aligned}
$$

donde $\mathbb{S}=\mathbb{C}^{-1}$ es el tensor flexibilidad del sólido sin polarizar, $\hat{\boldsymbol{\kappa}}=\boldsymbol{\kappa}-\mathbf{h}^{T}$ Sh, y el superíndice $T$ en el caso de los tensores de tercer orden denota la transposición entre el primer par de índices y el último.

Los parámetros materiales empleados típicamente para modelar cerámicos ferroeléctricos son tales que se verifica que $O(\|\mathbb{C}\| \times\|\boldsymbol{\kappa}\|)>>O\left(\|\mathbf{h}\|^{2}\right)$, y por lo tanto, es usual considerar que

$$
\hat{\mathrm{S}} \approx \mathbb{S}, \quad \hat{\boldsymbol{\kappa}} \approx \boldsymbol{\kappa}, \quad \hat{\boldsymbol{\epsilon}} \approx \boldsymbol{\epsilon}=\epsilon_{0} \mathbf{I}+\boldsymbol{\kappa}^{-1}, \quad \hat{\mathbf{h}} \approx \mathbb{S} \mathbf{h} \boldsymbol{\kappa}^{-1}
$$

lo que simplifica las expresiones (2.38)-(2.40) considerablemente. Las relaciones constitutivas acopladas toman entonces la forma

$$
\boldsymbol{\varepsilon}=\mathbb{S}^{(r)} \boldsymbol{\sigma}+\hat{\boldsymbol{\varepsilon}}^{(r)}-\hat{\mathbf{h}}^{(r)} \mathbf{E} \quad \mathrm{y} \quad \mathbf{D}=\boldsymbol{\epsilon}^{(r)} \mathbf{E}+\mathrm{p}-\hat{\mathbf{h}}^{(r) T} \boldsymbol{\sigma},
$$

mientras que la ley de evolución de la polarización irreversible toma la forma

$$
\begin{gathered}
\mathbf{E}-h_{0}^{(r)} \frac{\mathrm{p}}{1-\frac{|\mathrm{p}|}{p_{s}^{(r)}}}+\frac{3 \varepsilon_{s}^{(r)}}{\left(p_{s}^{(r)}\right)^{2}} \boldsymbol{\sigma}_{d} \mathrm{p}-\frac{1}{|\mathrm{p}|}\left[\left[\boldsymbol{\sigma} \cdot \hat{\mathbf{h}}^{(r)}(\mathrm{p}) \mathbf{E}\right] \frac{\mathrm{p}}{|\mathrm{p}|}+\right. \\
\left.+\frac{|\mathrm{p}|}{p_{s}^{(r)}}\left(\mathbf{I}-\frac{\mathrm{p}}{|\mathrm{p}|} \otimes \frac{\mathrm{p}}{|\mathrm{p}|}\right) \frac{\mathbf{v}}{\kappa^{(r)}}\right]=\left[e_{c}^{(r)}+e_{0}^{(r)}\left|\frac{\dot{\mathrm{p}}}{\dot{p}_{0}^{(r)}}\right|^{m}\right] \frac{\dot{\mathrm{p}}}{|\dot{\mathrm{p}}|}
\end{gathered}
$$

donde $\boldsymbol{\sigma}_{d}$ es la parte desviadora del tensor de tensiones y el vector $\mathbf{v}$ está dado por

$$
\begin{array}{r}
\mathbf{v}=2 \alpha_{0}\left(\frac{\mathrm{p}}{|\mathrm{p}|} \cdot \mathbf{E}\right)\left(\mathbb{S}^{(r)} \boldsymbol{\sigma}\right) \frac{\mathrm{p}}{|\mathrm{p}|}+\alpha_{0}\left(\frac{\mathrm{p}}{|\mathrm{p}|} \cdot\left(\mathbb{S}^{(r)} \boldsymbol{\sigma}\right) \frac{\mathrm{p}}{|\mathrm{p}|}\right) \mathbf{E}+\alpha_{\perp} \operatorname{tr}\left(\mathbb{S}^{(r)} \boldsymbol{\sigma}\right) \mathbf{E}+ \\
+2 \alpha_{\|}\left(\mathbb{S}^{(r)} \boldsymbol{\sigma}\right) \mathbf{E} .
\end{array}
$$

iii) En la práctica, muchos estudios micromecánicos de la respuesta piezoeléctrica de materiales compuestos ferroeléctricos asumen que la polarización irreversible es uniforme y constante en todo el sólido (por ejemplo, Dunn y Taya, 1993; Spinelli y 
Lopez-Pamies, 2014). Esta aproximación de polarización uniforme equivale a tomar

$$
\mathfrak{e}(\varepsilon, \mathbf{P}, \mathrm{p})=\frac{1}{2} \varepsilon \cdot \mathbb{C} \boldsymbol{\varepsilon}+\frac{1}{2} \mathbf{P} \cdot \boldsymbol{\kappa} \mathbf{P}+\boldsymbol{\varepsilon} \cdot \mathbf{h P} \quad \text { y } \quad \varphi(\dot{\mathrm{p}})=\left\{\begin{array}{ll}
0 & \dot{\mathrm{p}}=0 \\
+\infty & \text { c.c. }
\end{array},\right.
$$

con

$$
\mathbf{h}=\alpha_{0} \mathbf{n} \otimes \mathbf{n} \otimes \mathbf{n}+\alpha_{\perp} \mathbf{I} \otimes \mathbf{n}+\alpha_{=} \mathbf{n} \otimes_{s} \mathbf{I}
$$

donde $\boldsymbol{\varepsilon}$ y $\mathbf{P}$ son referidos a la configuración de referencia del sólido completamente polarizado y el vector unitario $\mathbf{n}$ denota la dirección de la polarización remanente. La densidad de energía complementaria está entonces dada por

$$
\psi(\boldsymbol{\sigma}, \mathbf{E}, \mathrm{p})=\frac{1}{2} \boldsymbol{\sigma} \cdot \hat{\mathrm{S}} \boldsymbol{\sigma}+\frac{1}{2} \mathbf{E} \cdot \hat{\boldsymbol{\epsilon}} \mathbf{E}-\boldsymbol{\sigma} \cdot \hat{\mathbf{h}} \mathbf{E},
$$

donde los tensores circunflejos están dados por expresiones análogas a (2.38)-(2.40), y pueden aproximarse por las expresiones (2.41).

Con las funciones termodinámicas completamente especificadas, las predicciones para cualquier historia de carga electromecánica pueden obtenerse mediante una evaluación sucesiva de las relaciones incrementales (2.19). Sin embargo, debe mencionarse aquí que, aunque el potencial de disipación (2.36) es una función convexa de la tasa de polarización irreversible p p, la energía interna (2.31) es una función no convexa de la polarización irreversible p, y entonces, la teoría constitutiva no se ajusta al modelo de material estándar generalizado (Germain et al., 1983). Consecuentemente, se espera que surjan inestabilidades numéricas en las relaciones incrementales (2.19) y en la unicidad de la polarización irreversible que maximiza el problema.

\subsection{Correspondencia con el ferromagnetismo}

A pesar de que los procesos atómicos detrás de los fenómenos de ferroelectricidad y ferromagnetismo son diferentes, la respuesta macroscópica de los sólidos ferromagnéticos tiene un paralelismo notable con la de los sólidos ferroeléctricos. En vistas de esta observación, Miehe et al. (2011a) propusieron una teoría fenomenológica de ferromagnetismo que es completamente análoga a la teoría de ferroelectricidad presentada anteriormente en la sección 2.2.1. Se basa en las siguientes reglas de correspondencia entre las descripciones matemáticas de los fenómenos eléctricos y magnéticos:

$$
\mathbf{E} \leftrightarrow \mathbf{H}, \quad \mathrm{D} \leftrightarrow \mathbf{B}, \quad \mathbf{P} \leftrightarrow \mathbf{M}, \quad \mathrm{p} \leftrightarrow \mathbf{m}
$$


Aquí, $\mathbf{H}$ es la intensidad del campo magnético, $\mathbf{B}$ es la inducción magnética, $\mathbf{M}$ es la magnetización del sólido, y $\mathbf{m}$ es un vector de magnetización irreversible que caracteriza el valor y orientación promedio de los dipolos magnéticos permanentes.

La energía interna y el potencial de disipación se consideran de la misma forma que sus homólogos ferroeléctricos y se escriben como

$$
\begin{array}{r}
\mathfrak{e}(\boldsymbol{\varepsilon}, \mathbf{M}, \mathbf{m})=\frac{1}{2}(\varepsilon-\hat{\boldsymbol{\varepsilon}}) \cdot \mathbb{C}(\boldsymbol{\varepsilon}-\hat{\boldsymbol{\varepsilon}})+\frac{1}{2}(\mathbf{M}-\mathbf{m}) \cdot \boldsymbol{\beta}^{-1}(\mathbf{M}-\mathbf{m})+ \\
(\boldsymbol{\varepsilon}-\hat{\boldsymbol{\varepsilon}}) \cdot \mathbf{h}(\mathbf{m})(\mathbf{M}-\mathbf{m})+\mathfrak{e}_{p d}(\mathbf{m})
\end{array}
$$

$\mathrm{y}$

$$
\varphi(\dot{\mathbf{m}})=h_{c}|\dot{\mathbf{m}}|+\frac{h_{0} \dot{m}_{0}}{1+n}\left(\frac{|\dot{\mathbf{m}}|}{\dot{m}_{0}}\right)^{1+n}
$$

donde $\boldsymbol{\beta}=\beta \mathbf{I}$ es el tensor de polarizabilidad magnética del sólido, $\mathrm{y}$

$$
\begin{aligned}
\mathbf{h}(\mathbf{m}) & =\frac{|\mathbf{m}|}{m_{s}}\left(\alpha_{0} \frac{\mathbf{m}}{|\mathbf{m}|} \otimes \frac{\mathbf{m}}{|\mathbf{m}|} \otimes \frac{\mathbf{m}}{|\mathbf{m}|}+\alpha_{\perp} \mathbf{I} \otimes \frac{\mathbf{m}}{|\mathbf{m}|}+\alpha_{=} \frac{\mathbf{m}}{|\mathbf{m}|} \otimes_{s} \mathbf{I}\right), \\
\mathfrak{e}_{p d}(\mathbf{m}) & =-h_{s} m_{s}^{2}\left[\ln \left(1-\frac{|\mathbf{m}|}{m_{s}}\right)+\frac{|\mathbf{m}|}{m_{s}}\right], \quad \mathrm{y} \\
\hat{\boldsymbol{\varepsilon}}(\mathbf{m}) & =\frac{3}{2} \varepsilon_{s}\left(\frac{|\mathbf{m}|}{m_{s}}\right)^{\gamma} \frac{\mathbf{m}}{|\mathbf{m}|} \otimes_{d} \frac{\mathbf{m}}{|\mathbf{m}|}
\end{aligned}
$$

denotan, respectivamente, el tensor piezomagnético, la energía almacenada y la deformación debido a los dipolos permanentes. En estas expresiones, $h_{c}$ es la intensidad del campo magnético coercitiva necesaria para activar la conmutación de los dipolos, $h_{0}$ y $\dot{m}_{0}$ son el campo magnético de referencia y la tasa de magnetización que caracteriza la dependencia del proceso de conmutación con la tasa, y $m_{s}$ es la magnetización en saturación. El resto de los parámetros materiales preservan el significado descrito en la sección anterior.

En base a esta correspondencia, el modelo desarrollado para materiales ferroeléctricos puede ser aplicado sin mayores modificaciones para obtener la respuesta de materiales ferromagnéticos. Si bien ya se ha mencionado que el estudio de este tipo de materiales excede los alcances de este trabajo, se presenta aquí para evaluar el potencial de los modelos para resolver sistemas materiales más complejos, como pueden ser compuestos ferroeléctricos con inclusiones ferromagnéticas en los denominados sistemas multiferróicos. 


\section{Evaluación de la teoría}

\subsection{Introducción}

En base a la teoría fenomenológica y las densidades de energía definidas en el capítulo 2, es posible generar predicciones para sólidos ferróicos sometidos a la acción simultánea de campos eléctricos y cargas mecánicas. Durante el desarrollo de la teoría fenomenológica, en pos de simplificar la formulación matemática del fenómeno, se ha limitado la capacidad para considerar la conmutación de dominios inducida por estados de cargas mecánicos. Si bien esto implica que deberían obtenerse predicciones adecuadas para estados de carga donde predominen los campos eléctricos/magnéticos, se espera que dentro de los rangos físicamente admisibles de tensiones y campos eléctricos/magnéticos las estimaciones de la respuesta sean confiables. Se presentan aquí predicciones obtenidas con esta teoría de medios continuos para materiales ferroeléctricos y ferromagnéticos homogéneos bajo distintas condiciones de carga. Se analizan las capacidades del modelo para reproducir adecuadamente el comportamiento del material y se discuten posibles limitaciones para su aplicabilidad en los diversos sistemas materiales.

\subsection{Predicciones para sólidos ferroeléctricos}

En la Tabla 3.1 se presentan los valores numéricos adoptados para los diversos parámetros materiales requeridos para simular sólidos ferroeléctricos. Estos valores reproducen aproximadamente el comportamiento sensible a la tasa del titanato zirconato de plomo policristalino a bajas frecuencias (Miehe y Rosato, 2011; Zhou et al., 2001) y cumplen con las condiciones (2.33) de convexidad para la energía libre de Helmholtz. El exponente de la deformación irreversible comúnmente se considera en la literatura como $\gamma=1$ o $\gamma=2$, por lo que ambos valores serán utilizados.

Para cada paso de tiempo, el potencial incremental (2.20) se calcula maximizando la función $J$ con un algoritmo de búsqueda directa compleja para funciones suaves a trozos y la respuesta material se obtiene evaluando las expresiones (2.19) en la polarización irreversible óptima. El paso de tiempo fue seleccionado a partir de un análisis de convergencia, en el cual se evaluó la solución del problema para distintos pasos de tiempo. A modo de ejemplo se presentan en la figura 3.1 curvas de histéresis para tres intervalos de tiempo distintos. Comparando los resultados obtenidos, se selecciona el paso de tiempo que será utilizado en los cálculos como $\Delta t=10^{-3} \mathrm{~s}$. 
Tabla 3.1: Parámetros materiales adoptados para el sólido ferroeléctrico. Los valores reproducen aproximadamente el comportamiento sensible a la tasa de titanato zirconato de plomo policristalino a bajas frecuencias (Miehe y Rosato, 2011; Zhou et al., 2001).

\begin{tabular}{clcr}
\hline Símbolo & Parámetro & Unidades & Valor \\
\hline$\kappa^{-1}$ & polarizabilidad eléctrica & $\mathrm{C} /(\mathrm{V} \cdot \mathrm{m})$ & $1800 \epsilon_{0}$ \\
$p_{s}$ & polarización de saturación & $\mathrm{C} / \mathrm{m}^{2}$ & 0.25 \\
$h_{s}$ & pendiente de histéresis & $\mathrm{MV} \cdot \mathrm{m} / \mathrm{C}$ & 0.1 \\
$n$ & exponente sensible a la tasa & - & 0.2 \\
$\dot{p}_{0}$ & tasa de polarización de referencia & $\mathrm{C} /\left(\mathrm{m}^{2} \cdot \mathrm{s}\right)$ & 100 \\
$e_{c}$ & campo eléctrico coercitivo & $\mathrm{MV} / \mathrm{m}$ & 0.35 \\
$e_{0}$ & campo eléctrico de referencia & $\mathrm{MV} / \mathrm{m}$ & 0.35 \\
$\gamma$ & exponente de deformación irreversible & - & 1 \\
$\mu$ & módulo de Lamé & $\mathrm{GPa}$ & 45 \\
$\lambda$ & módulo de Lamé & $\mathrm{GPa}$ & 70 \\
$\varepsilon_{s}$ & deformación de saturación & - & 0.001 \\
$\alpha_{0}$ & expansión axial piezoeléctrica & $\mathrm{MN} / \mathrm{C}$ & 12,6 \\
$\alpha_{\perp}$ & expansión lateral piezoeléctrica & $\mathrm{MN} / \mathrm{C}$ & 276 \\
$\alpha_{=}$ & distorsión piezoeléctrica & $\mathrm{MN} / \mathrm{C}$ & -1460 \\
\hline
\end{tabular}

\subsubsection{Estado de carga eléctrico uniaxial}

En primer lugar se considera un espécimen sin polarizar sometido a una solicitación eléctrica en ausencia de cargas mecánicas. Se aplica una señal eléctrica triangular $E(t)$ con una amplitud pico de $4 e_{c}$ y una frecuencia de $1 \mathrm{~Hz}$, como se observa en la figura 3.2 .

a)

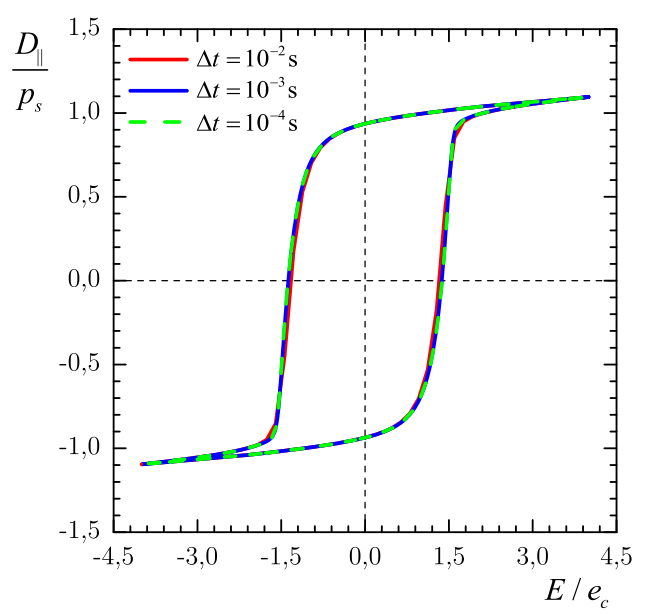

b)

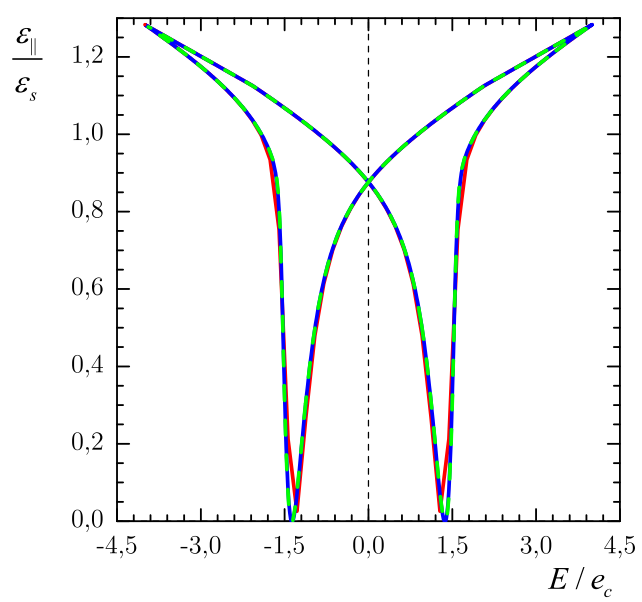

Figura 3.1: Comparación de las predicciones para especímenes ferroeléctricos libres de deformar con exponentes de deformación irreversible $\gamma=2$ para distintos intervalos de tiempo: a) desplazamiento eléctrico $\left(D_{\|}\right)$y b) deformación longitudinal $\left(\varepsilon_{\|}\right)$a lo largo de la dirección del campo eléctrico aplicado. 
a)

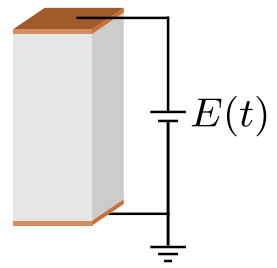

b)

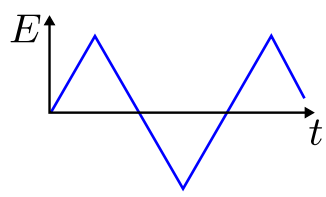

Figura 3.2: Representación esquemática del estado de carga eléctrico para los especímenes: a) Carga uniaxial; b) Perfil de carga.

En la figura 3.3 se presentan predicciones para especímenes con $\gamma=1$ y $\gamma=2$, respectivamente, en la forma de gráficos de desplazamiento eléctrico $\left(D_{\|}\right)$y de deformación axial $\left(\varepsilon_{\|}\right)$en la dirección de la carga aplicada en función de la intensidad del campo eléctrico. Se muestran también en estas figuras las curvas correspondientes a los valores picos de las componentes de la polarización irreversible en la dirección paralela $\left(\mathrm{p}_{\|}=\mathrm{p} \cdot \mathbf{n}\right)$ y perpendicular $\left(\mathrm{p}_{\perp}=\left|\mathrm{p}_{\perp}\right|=\left|\mathrm{p}-\mathrm{p}_{\|} \mathbf{n}\right|\right)$ a la dirección de aplicación de la carga en función del número de ciclos de carga. La respuesta transitoria inicial y la respuesta estabilizada se indican en lineas punteadas y continuas, respectivamente. En primer lugar, se observa que la respuestas predichas por ambos exponentes de deformación irreversible son consistentes con las observaciones experimentales (Zhou et al., 2001). Las predicciones reproducen la respuesta de ciclos de histéresis eléctrica típicamente observados en ferrocerámicos. Se observa que el exponente $\gamma=2$ genera respuestas más redondeadas que el exponente $\gamma=1$, pero más allá de eso, las respuestas son cualitativamente similares. La polarización irreversible subyacente se mantiene alineada con la dirección de las cargas aplicadas, tal y como debería ser en vista de la isotropía inicial del material, y la respuesta se estabiliza inmediatamente después de la primera rampa de carga.

\subsubsection{Estado de carga eléctrico multiaxial}

Se considera ahora un caso que produce rotación de la polarización remanente, tal como se observa en los resultados experimentales de Huber y Fleck (2001). En estos experimentos se polarizó una placa de PZT, a la cual posteriormente se le removieron los electrodos. A partir de esta, se cortaron especímenes sobre los cuales se aplicaron electrodos nuevamente, de modo que el campo eléctrico pudiese ser impuesto en un ángulo relativo a la polarización preexistente. A medida que el campo eléctrico aumenta, la polarización remanente rota de forma tal que el ángulo entre la dirección de la polarización y del campo eléctrico se reduce. En los casos en los cuales el ángulo inicial era obtuso, el espécimen debe primero despolarizarse para luego repolarizarse en dirección paralela al campo eléctrico aplicado. 

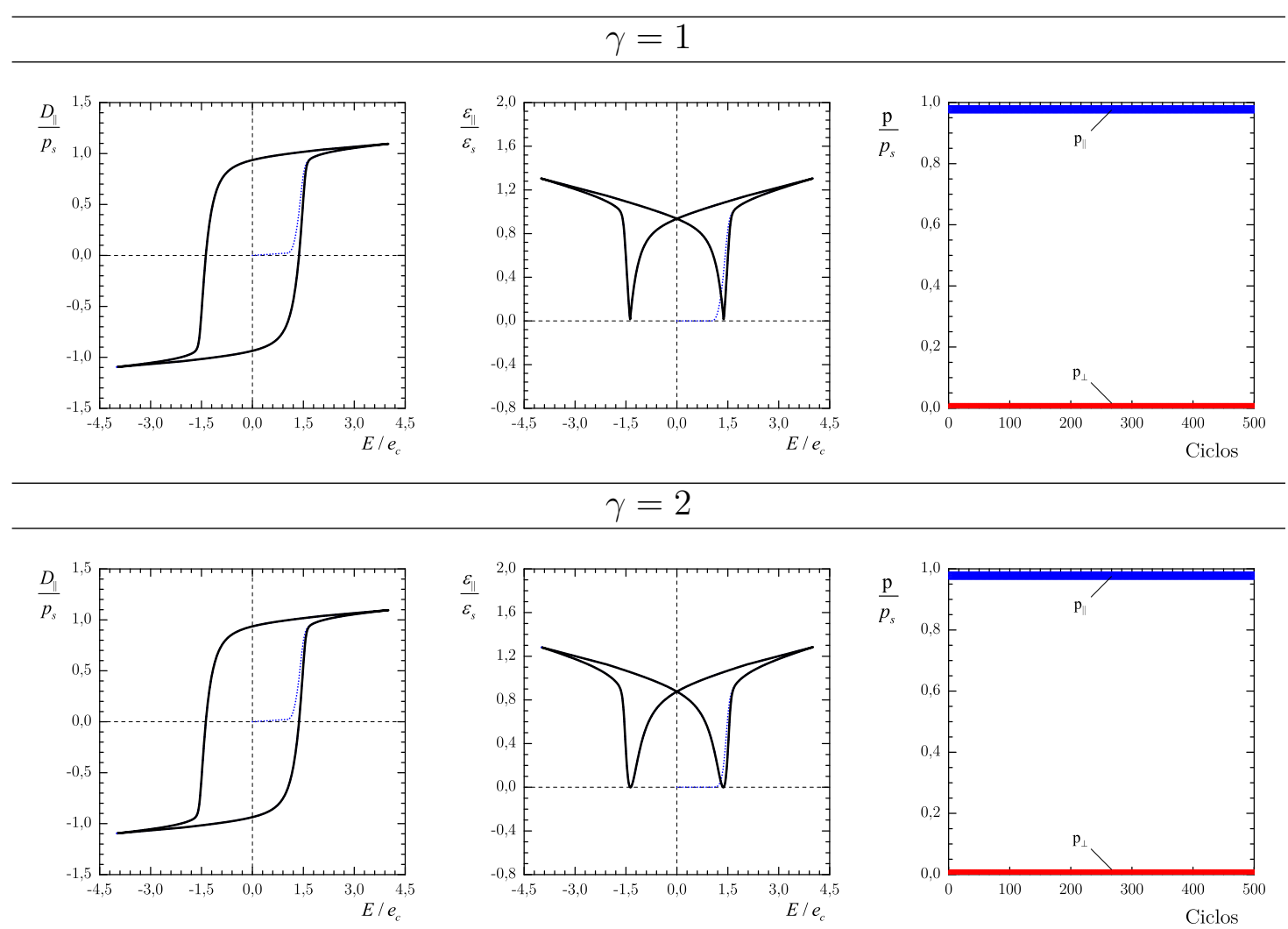

Figura 3.3: Predicciones para especímenes ferroeléctricos sin cargas mecánicas con exponentes de deformación irreversible $\gamma=1$ y $\gamma=2$ : desplazamiento eléctrico $\left(D_{\|}\right)$y deformación longitudinal $\left(\varepsilon_{\|}\right)$a lo largo de la dirección del campo eléctrico aplicado, y componentes de polarización irreversible paralela $\left(\mathrm{p}_{\|}\right)$y perpendicular $\left(\mathrm{p}_{\perp}\right)$.

Para obtener las predicciones de este caso de carga, se considera entonces un espécimen como el de la sección anterior sometido a un campo eléctrico uniaxial, en ausencia de cargas mecánicas y asumiendo que se encuentra polarizado en diferentes direcciones relativas al campo eléctrico aplicado. La magnitud del campo eléctrico aumenta linealmente hasta un valor de $4 e_{c}$. Los resultados se presentan en la figura 3.4 para exponentes de deformación irreversible $\gamma=1$ y $\gamma=2$. Se muestra el cambio en el desplazamiento eléctrico $\left(\Delta D_{\|}\right)$y la deformación $\left(\Delta \varepsilon_{\|}\right)$en la dirección del campo eléctrico aplicado.

Puede observarse que para un ángulo inicial de $180^{\circ}$, la curva corresponde a medio ciclo de histéresis como los presentados en la figura 3.3, mientras que para el caso del ángulo inicial de $0^{\circ}$ la respuesta es prácticamente lineal. Esto último se produce porque el material ha alcanzado la saturación y el aumento del campo eléctrico simplemente lo conduce más hacia esa condición. Para los ángulos intermedios, el comportamiento implica una transición progresiva entre el medio ciclo de histéresis y la respuesta lineal. Este mismo comportamiento es el que se observa en los resultados experimentales de Huber y Fleck (2001). 

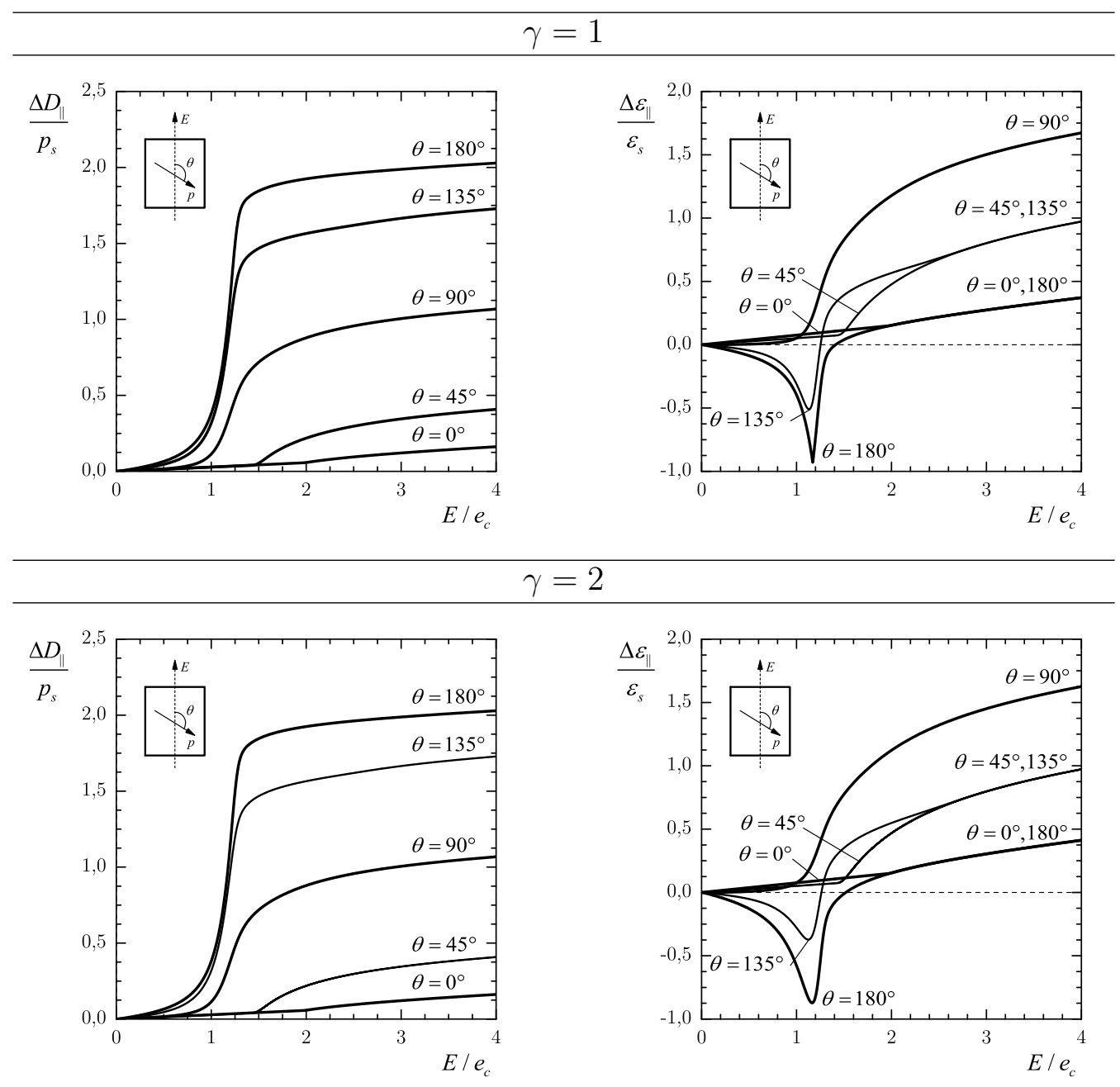

Figura 3.4: Curvas de rotación de la polarización. a) Variación del desplazamiento eléctrico en la dirección del campo aplicado y b) variación de la deformación en la dirección del campo aplicado en función de la intensidad de campo eléctrico aplicado.

\subsubsection{Estado de carga electromecánico uniaxial}

Se considera ahora que los especímenes sin polarizar son sometidos a una tensión mecánica $\sigma$ en una cierta dirección, y luego son sometidos a una señal eléctrica triangular $E(t)$ en la misma dirección con una amplitud pico de $4 e_{c} \mathrm{y}$ una frecuencia de $1 \mathrm{~Hz}$, manteniendo el nivel de tensión constante, como se observa en las figuras 3.5a,b.

En la figura 3.6 se presentan predicciones para especímenes traccionados a un nivel de 50MPa mientras que en la figura 3.7 se presentan predicciones para especímenes comprimidos a un nivel de -50MPa. En ambos casos se utilizan los exponentes de deformación $\gamma=1$ y $\gamma=2$. Nuevamente los resultados se muestran en la forma de gráficos de desplazamiento eléctrico $\left(D_{\|}\right)$y de deformación axial $\left(\varepsilon_{\|}\right)$en la dirección de la carga aplicada en función de la intensidad del campo eléctrico, así como también mediante curvas de evolución de la polarización irreversible en función del número de 
a)

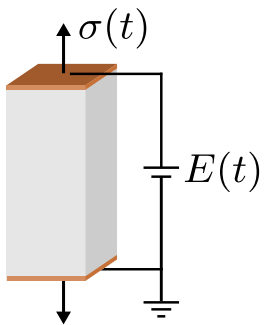

b)

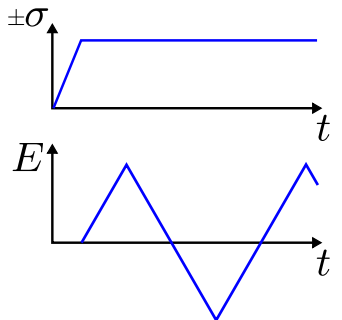

c)

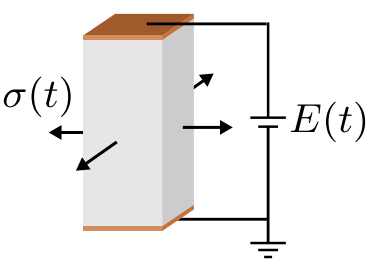

Figura 3.5: Representación esquemática del estado de carga electromecánico para los especímenes: a) Carga uniaxial; b) Perfil de carga; c) Carga no uniaxial.

ciclos de carga. La respuesta transitoria inicial y la respuesta estabilizada se indican en lineas punteadas y continuas, respectivamente. Los niveles de tensión considerados son del orden de los niveles que puede soportar el PZT (Munz et al., 1998).

En las respuestas predichas para los especímenes traccionados no se observan cambios cualitativos con respecto a la respuesta obtenida en ausencia de tensiones mecánicas en la subsección 3.2.1. Los ciclos de histéresis eléctrica se ensanchan ligeramente, por lo que se incrementa el nivel de polarización remanente y el valor del campo
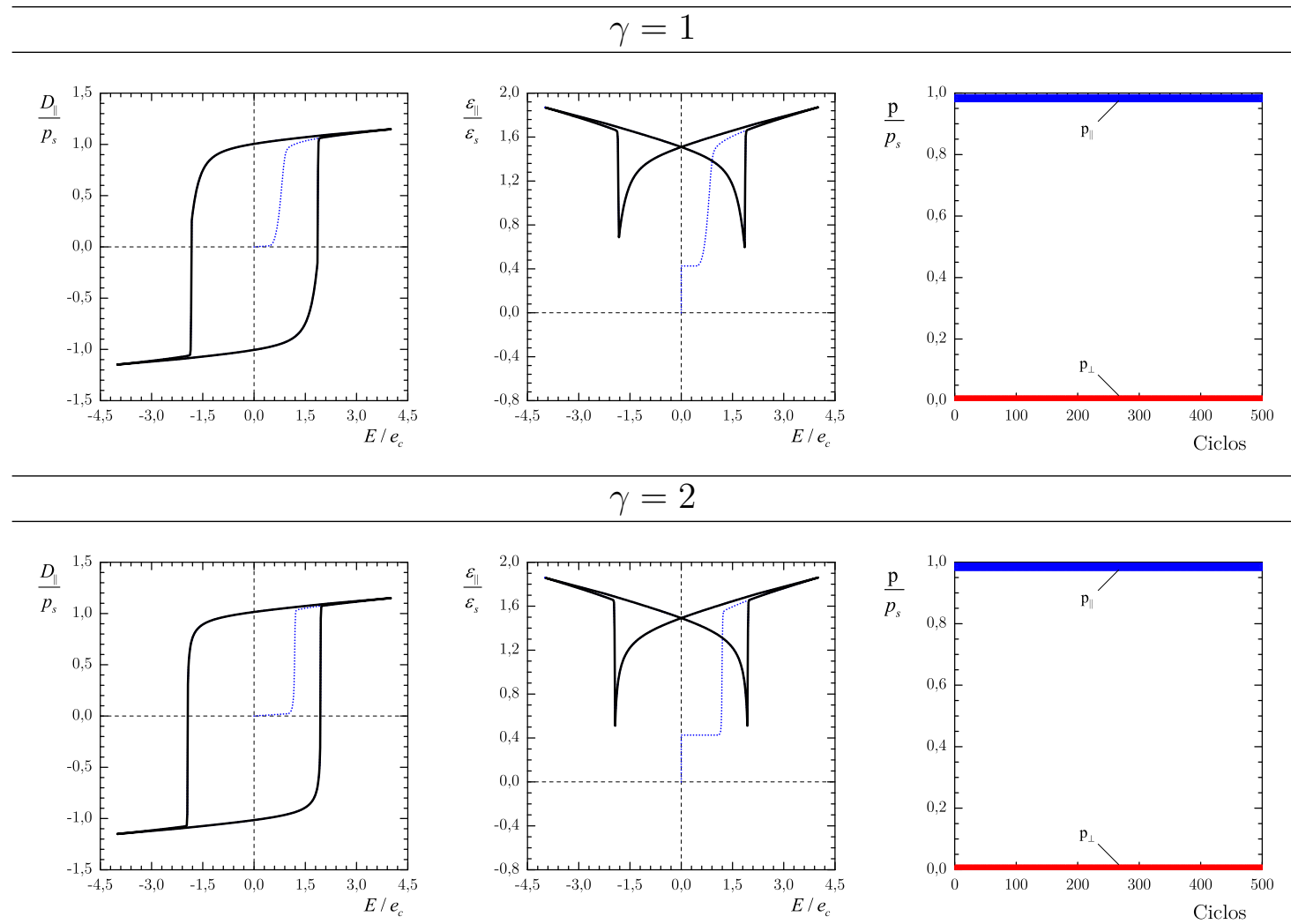

Figura 3.6: Predicciones para especímenes ferroeléctricos sometidos a tracción longitudinal de 50MPa con exponentes de deformación irreversible $\gamma=1$ y $\gamma=2$ : desplazamiento eléctrico $\left(D_{\|}\right)$y deformación longitudinal $\left(\varepsilon_{\|}\right)$a lo largo de la dirección del campo eléctrico aplicado, y componentes de polarización irreversible paralela $\left(\mathrm{p}_{\|}\right)$y perpendicular $\left(\mathrm{p}_{\perp}\right)$. 
$\gamma=1$
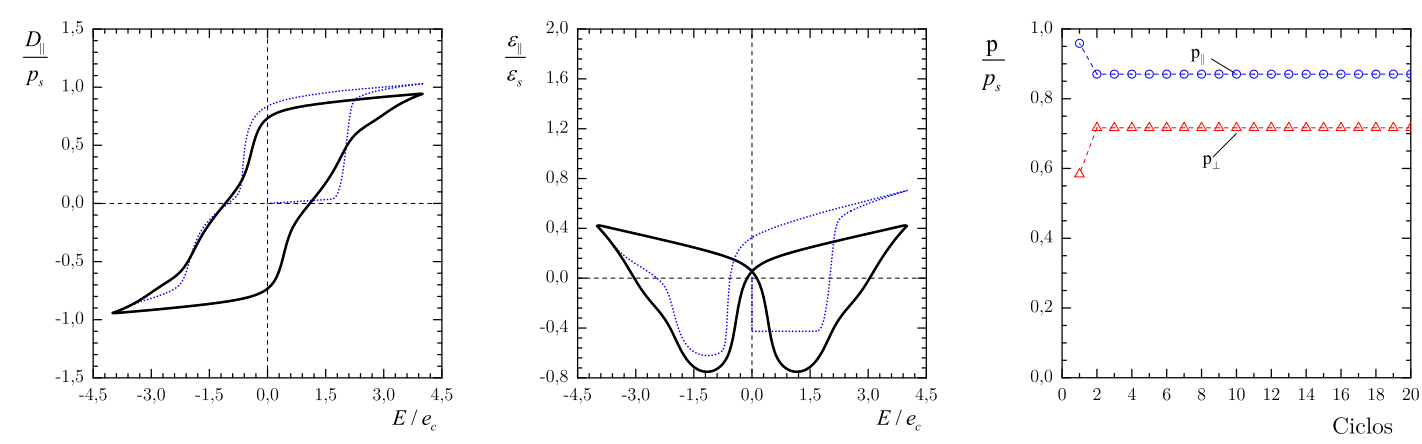

$\gamma=2$
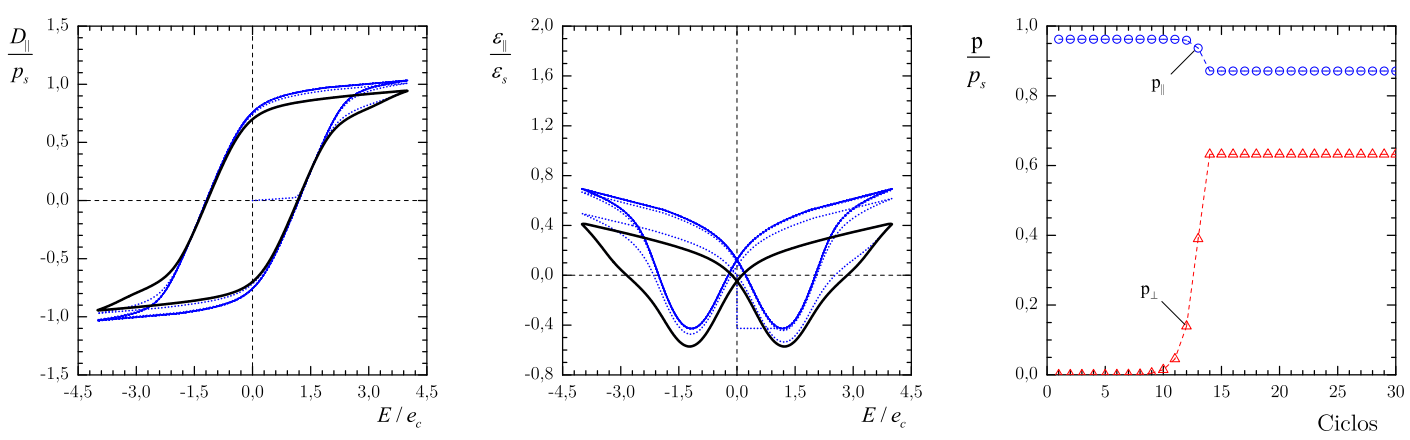

Figura 3.7: Predicciones para especímenes ferroeléctricos sometidos a compresión longitudinal de -50MPa con exponentes de deformación irreversible $\gamma=1$ y $\gamma=2$ : desplazamiento eléctrico $\left(D_{\|}\right)$y deformación longitudinal $\left(\varepsilon_{\|}\right)$a lo largo de la dirección del campo eléctrico aplicado, y componentes de polarización irreversible paralela $\left(\mathrm{p}_{\|}\right)$y perpendicular $\left(\mathrm{p}_{\perp}\right)$.

coercitivo. A su vez, los ciclos de histéresis de deformación se desplazan debido a la deformación mecánica inicial y se tornan más angulosos. Al igual que para el caso puramente eléctrico, la polarización remanente se mantiene alineada con la dirección de las cargas aplicadas debido a la simetría inicial del problema. Las respuestas predichas para especímenes comprimidos, por otro lado, aparentan no respetar la física del problema luego de un número determinado de ciclos de carga. En efecto, se observan transitorios más largos y la polarización irreversible estabilizada no se encuentra alineada con la dirección de las cargas aplicadas. Aunque este tipo de características pueden surgir en muestras monocristalinas pequeñas (véase, por ejemplo, Zhang y Bhattacharya, 2005), no son esperables en muestras policristalinas altamente desordenadas como las que se consideran en el modelo utilizado (véase, por ejemplo, Zhou et al., 2005). Esto resulta ser una seria falencia de la teoría, consecuencia de la dependencia no convexa de la densidad de energía interna asumida (2.31) con respecto a la polarización irreversible. Esta no convexidad es introducida por el primer y tercer término de la energía interna, que representan las contribuciones elástica y piezoeléctrica. La fuente del problema es causada por el primer término, hecho que se observa despreciando el efecto piezoeléctrico y la contribución de las 
polarización irreversible en los tensores flexibilidad $\widehat{\mathbb{S}}$ y permitividad $\hat{\boldsymbol{\epsilon}}$, con lo que la función objetivo (2.21) siendo minimizada con respecto a p en cada paso de tiempo está dada por

$$
\begin{aligned}
& J \approx \frac{\sigma^{2}}{2 Y}+\frac{\epsilon E^{2}}{2}+E \mathrm{p}_{\|}-\frac{\sigma \varepsilon_{s}}{2}\left(\frac{|\mathrm{p}|}{p_{s}}\right)^{\gamma}\left[1-3\left(\frac{\mathrm{p}_{\|}}{|\mathrm{p}|}\right)^{2}\right]-\Delta t e_{c}|\dot{\mathrm{p}}|+ \\
&+h_{s} p_{s}^{2}\left[\ln \left(1-\frac{|\mathrm{p}|}{p_{s}}\right)+\frac{|\mathrm{p}|}{p_{s}}\right]-\Delta t \frac{e_{0} \dot{p}_{0}}{1+n}\left(\frac{|\dot{\mathrm{p}}|}{\dot{p}_{0}}\right)^{1+n}
\end{aligned}
$$

donde $\sigma$ es el nivel de tensión aplicado, $Y$ y $\epsilon$ son el módulo de Young y la permitividad del sólido, $\dot{\mathrm{p}}=\left(\mathrm{p}-\mathrm{p}_{i}\right) / \Delta t,|\mathrm{p}|=\sqrt{\mathrm{p}_{\|}^{2}+\mathrm{p}_{\perp}^{2}}, \mathrm{y}_{\|} \mathrm{y}_{\perp}$ son las componentes de la polarización p paralela y perpendicular a la dirección de la carga. La ecuación de evolución $(2.12)_{3}$ para la polarización irreversible en la dirección perpendicular a la dirección de la carga aplicada, $\mathrm{p}_{\perp}$, es entonces

$$
-\left\{\frac{h_{s}}{1-|\mathrm{p}| / p_{s}}+\frac{\sigma \varepsilon_{s}}{2 p_{s}|\mathrm{p}|}\left[1+3\left(\frac{\mathrm{p}_{\|}}{|\mathrm{p}|}\right)^{2}\right]\right\} \mathrm{p}_{\perp}=\left[e_{c}+e_{0}\left|\frac{\dot{\mathrm{p}}}{\dot{p}_{0}}\right|^{n}\right] \frac{\dot{\mathrm{p}}_{\perp}}{|\dot{\mathrm{p}}|}, \quad \mathrm{p}_{\perp}(0)=0
$$

para el exponente $\gamma=1, \mathrm{y}$

$$
-\left\{\frac{h_{s}}{1-|\mathrm{p}| / p_{s}}+\frac{\sigma \varepsilon_{s}}{p_{s}^{2}}\right\} \mathrm{p}_{\perp}=\left[e_{c}+e_{0}\left|\frac{\dot{\mathrm{p}}}{\dot{p}_{0}}\right|^{n}\right] \frac{\dot{\mathrm{p}}_{\perp}}{|\dot{\mathrm{p}}|}, \quad \mathrm{p}_{\perp}(0)=0
$$

para el exponente $\gamma=2$.

La solución trivial $\mathrm{p}_{\perp}(t)=0$ para estas ecuaciones es numéricamente estable si los factores entre llaves multiplicando $\mathrm{p}_{\perp}$ en los términos del lado izquierdo son positivos. Si bien este es el caso para cualquier nivel de tensión de tracción $(\sigma>0)$, no es el caso para tensiones de compresión lo suficientemente grandes $(\sigma<0)$. En este último caso, es esperable que ocurra una acumulación exponencial de $\mathrm{p}_{\perp}(t)$. Esto está de acuerdo con los resultados observados en las figuras 3.6 y 3.7. Cabe destacar que $\mathrm{p}_{\perp}(t)$ toma un valor finito luego del primer ciclo de carga en los especímenes comprimidos con $\gamma=1$, mientras que lo mismo ocurre luego de varios ciclos de carga con $\gamma=2$. Lógicamente, el número de ciclos de carga requeridos para que $\mathrm{p}_{\perp}(t)$ tome un valor finito aumenta a medida que disminuye el nivel de tensión aplicado.

Nótese que el problema presentado es de carácter netamente multiaxial. Es por ello que en los trabajos de McMeeking y Landis (2002), en los cuales se restringe el análisis a casos uniaxiales, no se evidencian las inestabilidades presentadas aquí. 
$\gamma=1$
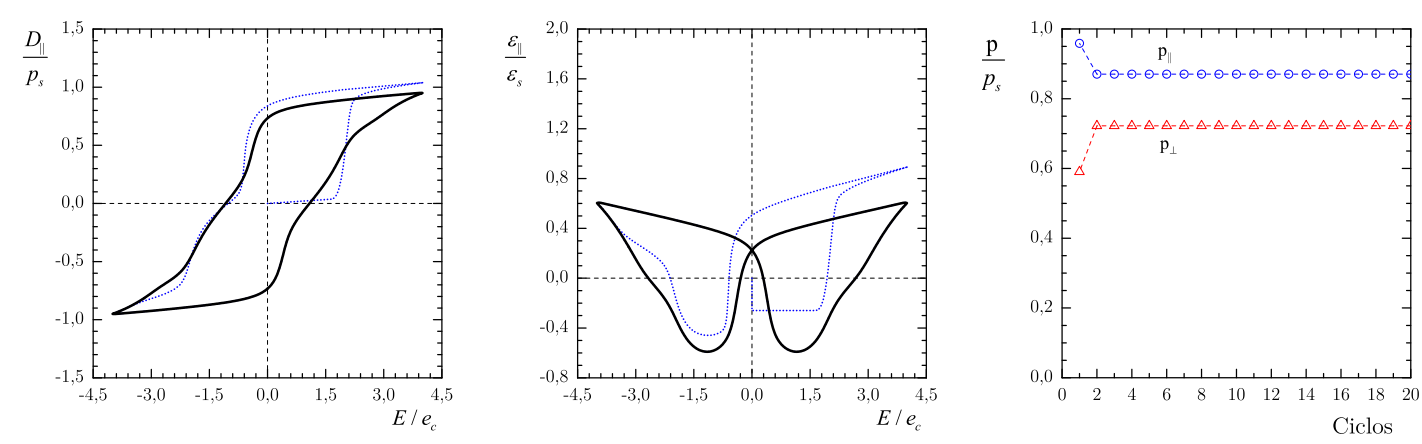

$\gamma=2$
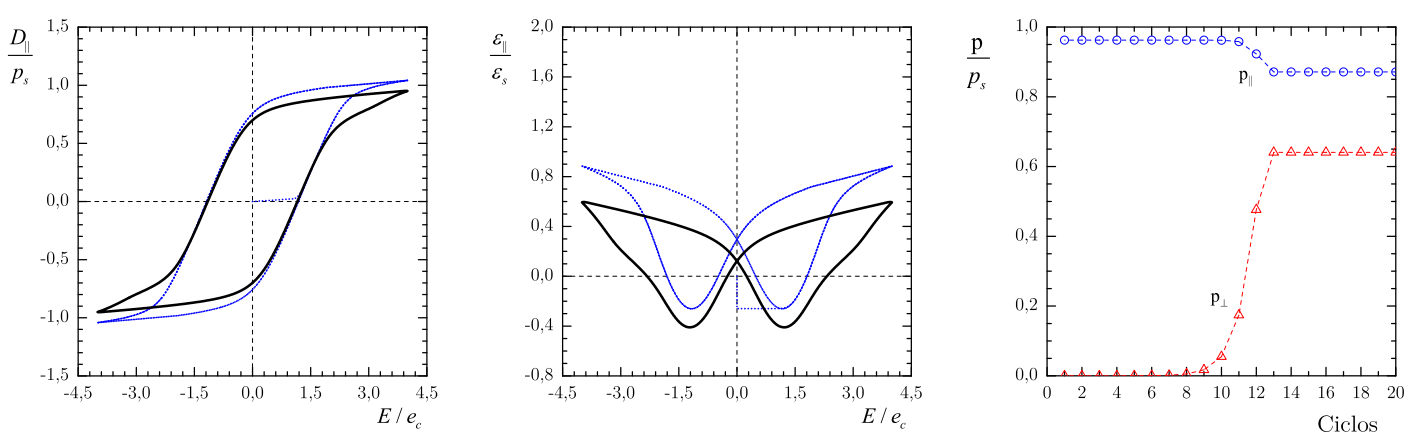

Figura 3.8: Predicciones para especímenes ferroeléctricos sometidos a tracción perpendicular con exponentes de deformación irreversible $\gamma=1$ y $\gamma=2$ : desplazamiento eléctrico $\left(D_{\|}\right)$y deformación longitudinal $\left(\varepsilon_{\|}\right)$a lo largo de la dirección del campo eléctrico aplicado, y componentes de polarización irreversible paralela $\left(\mathrm{p}_{\|}\right)$y perpendicular $\left(\mathrm{p}_{\perp}\right)$.

\subsubsection{Estado de carga electromecánico multiaxial}

Los problemas de estabilidad presentados anteriormente no se limitan únicamente a estados de carga uniaxiales. Considérese, por ejemplo, un espécimen sometido a la misma secuencia de carga que el caso anterior, pero con la tensión mecánica aplicada perpendicularmente a la dirección del campo eléctrico, como se observa en la figura 3.5c. En la figura 3.8 se presentan los resultados para especímenes traccionados y en la figura 3.9 para especímenes comprimidos. En ambos casos se utilizan los exponentes de deformación $\gamma=1$ y $\gamma=2$. Se observa que los resultados son similares a los correspondientes al estado de carga uniaxial, con la excepción de que ahora el cambio en la dirección de la polarización irreversible se presenta para los estados de carga de tracción perpendicular a la dirección del campo eléctrico aplicado. En vistas del carácter isocórico de $\hat{\boldsymbol{\varepsilon}}(\mathrm{p})$, las ecuaciones de evolución para la polarización irreversible perpendicular a la dirección del campo eléctrico aplicado están dadas por las mismas expresiones (3.2) y (3.3) pero con signo opuesto frente a la tensión $\sigma$. Por lo tanto, la solución trivial $\mathrm{p}_{\perp}(t)=0$ es ahora numéricamente estable para cualquier nivel de tensión de compresión $(\sigma<0)$ pero es inestable para valores suficientemente 

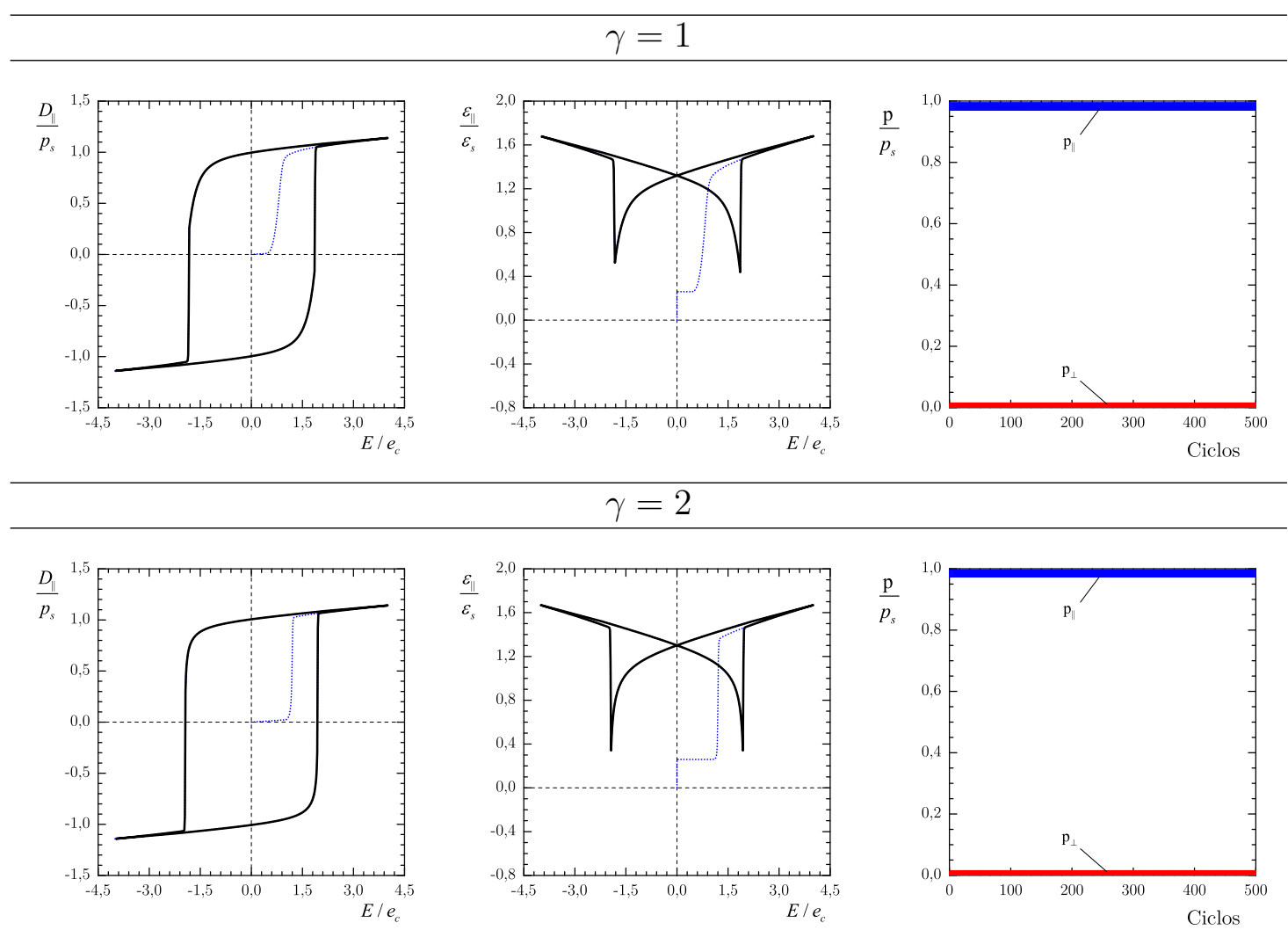

Figura 3.9: Predicciones para especímenes ferroeléctricos sometidos a compresión perpendicular con exponentes de deformación irreversible $\gamma=1$ y $\gamma=2$ : desplazamiento eléctrico $\left(D_{\|}\right)$y deformación longitudinal $\left(\varepsilon_{\|}\right)$a lo largo de la dirección del campo eléctrico aplicado, y componentes de polarización irreversible paralela $\left(\mathrm{p}_{\|}\right)$y perpendicular $\left(\mathrm{p}_{\perp}\right)$.

elevados de tensiones de tracción $(\sigma>0)$, confirmando los resultados obtenidos en las predicciones.

Los límites de estabilidad para condiciones de carga aleatoria surgen del conocimiento del Hessiano de la energía libre (2.31), que es bastante complicado. En cualquier caso, el análisis anterior sugiere que es de esperar que existan problemas de estabilidad cuando los especímenes sean ciclados bajo cargas eléctricas por encima de $e_{c}$ en combinación con niveles de tensión excediendo $\sim h_{s} p_{s}^{2} / \varepsilon_{s}$. Esos niveles de tensión son del orden de $5 \mathrm{MPa}$ para los casos considerados aquí. Por lo tanto, a pesar de que algunos impulsores de esta teoría afirman que está destinada únicamente a estados de carga con niveles bajos de tensión — por ejemplo, McMeeking y Landis (2002)-, ésta debe utilizarse con precaución incluso dentro del supuesto rango de validez.

\subsection{Predicciones para sólidos ferromagnéticos}

En la sección 2.3 se presentó una equivalencia de la teoría fenomenológica concebida originalmente para materiales ferroeléctricos para poder ser utilizada en el caso de materiales ferromagnéticos. Siguiendo los lineamientos de Miehe et al. (2011a), este 
Tabla 3.2: Parámetros materiales adoptados para el sólido ferromagnético. Los valores reproducen aproximadamente el comportamiento sensible a la tasa del Terfenol-D a bajas frecuencias (Miehe et al., 2011a). La constante $\mu_{0}$ denota la permeabilidad del vacío.

\begin{tabular}{clcr}
\hline Símbolo & Parámetro & Unidades & Valor \\
\hline$\beta$ & polarizabilidad magnética & $\mathrm{N} / \mathrm{A}^{2}$ & $3.3 \mu_{0}$ \\
$m_{s}$ & magnetización saturación & $\mathrm{N} /(\mathrm{A} \cdot \mathrm{m})$ & 0.25 \\
$h_{s}$ & pendiente de histéresis & $\mathrm{A}^{2} / \mathrm{N}$ & 800 \\
$m$ & exponente sensible a la tasa & - & 0.5 \\
$\dot{m}_{0}$ & tasa de magnetización de referencia & $\mathrm{MN} \cdot \mathrm{s} /(\mathrm{A} \cdot \mathrm{m})$ & 10 \\
$h_{c}$ & campo magnético coercitivo & $\mathrm{A} / \mathrm{m}$ & 2000 \\
$h_{v 0}$ & campo magnético de referencia & $\mathrm{A} / \mathrm{m}$ & 2000 \\
$\mu$ & módulo de Lamé & $\mathrm{GPa}$ & 10 \\
$\lambda$ & módulo de Lamé & $\mathrm{GPa}$ & 15 \\
$\varepsilon_{s}$ & deformación de saturación & - & 0.002 \\
$\alpha_{0}$ & expansión piezomagnética axial & $\mathrm{MA} / \mathrm{m}$ & $-17,7$ \\
$\alpha_{\perp}$ & expansión piezomagnética lateral & $\mathrm{MA} / \mathrm{m}$ & $-37,8$ \\
$\alpha_{=}$ & distorsión piezomagnética & $\mathrm{MA} / \mathrm{m}$ & 29,4 \\
\hline
\end{tabular}

desarrollo se utiliza entonces para generar predicciones para sólidos ferromagnéticos sometidos a la acción simultánea de campos magnéticos y cargas mecánicas. En la tabla 3.2 se muestran los valores numéricos utilizados para los diversos parámetros materiales. Estos valores reproducen aproximadamente el comportamiento sensible a la tasa de la aleación Terfenol-D a bajas frecuencias (Miehe et al., 2011a) y cumplen con las condiciones (2.33).

\subsubsection{Estado de carga magnética uniaxial}

En primer lugar, consideramos especímenes sin magnetizar y libres de tensión mecánica que se someten a una señal magnética triangular $H(t)$ a lo largo de una cierta dirección, con una amplitud pico de $4 h_{c}$ y una frecuencia de $1 \mathrm{~Hz}$. Para cada paso de carga, el potencial incremental (2.20) adaptado a las variables ferromagnéticas se calcula maximizando la función $J$ con un algoritmo de búsqueda directa compleja para funciones suaves a trozos y la respuesta material se obtiene evaluando las expresiones (2.19) en la magnetización irreversible óptima. El paso de tiempo empleado en los cálculos es $\Delta t=10^{-3} \mathrm{~s}$.

La figura 3.10 muestra las predicciones para especímenes con exponentes de deformación irreversible $\gamma=1$ y $\gamma=2$ mediante gráficos de la inducción magnética $\left(B_{\|}\right)$y la deformación axial $\left(\varepsilon_{\|}\right)$en la dirección del campo magnético aplicado en función de la intensidad del campo magnético $(H)$. Se muestran también curvas correspondientes a los valores pico de las componentes de magnetización irreversibles en la dirección paralela $\left(\mathrm{m}_{\|}=\mathbf{m} \cdot \mathbf{n}\right)$ y perpendicular $\left(\mathrm{m}_{\perp}=\left|\mathbf{m}_{\perp}\right|=\left|\mathbf{m}-\mathrm{m}_{\|} \mathbf{n}\right|\right)$ al campo aplicado 
$\gamma=1$
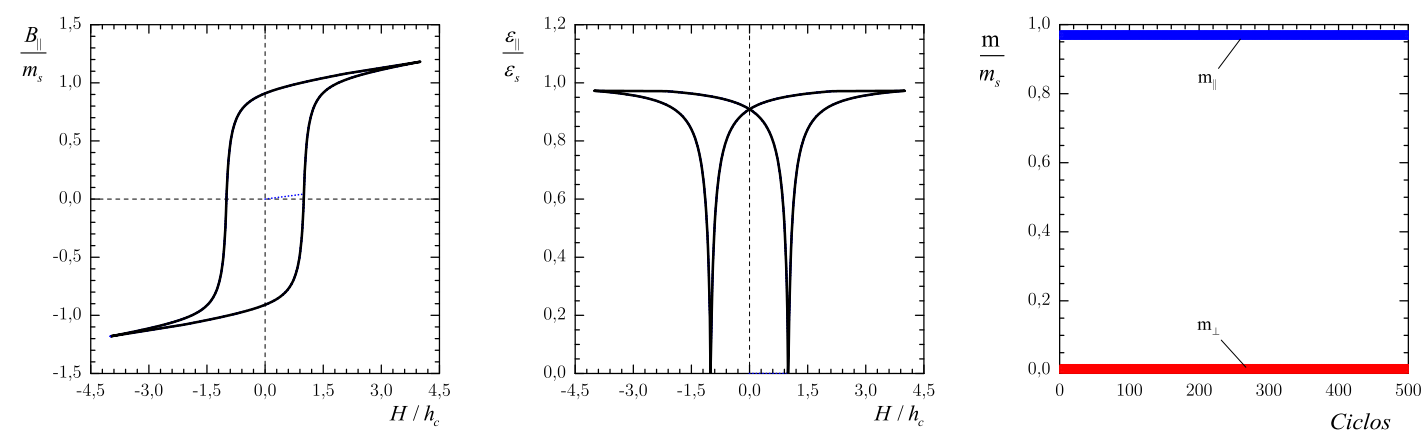

$\gamma=2$
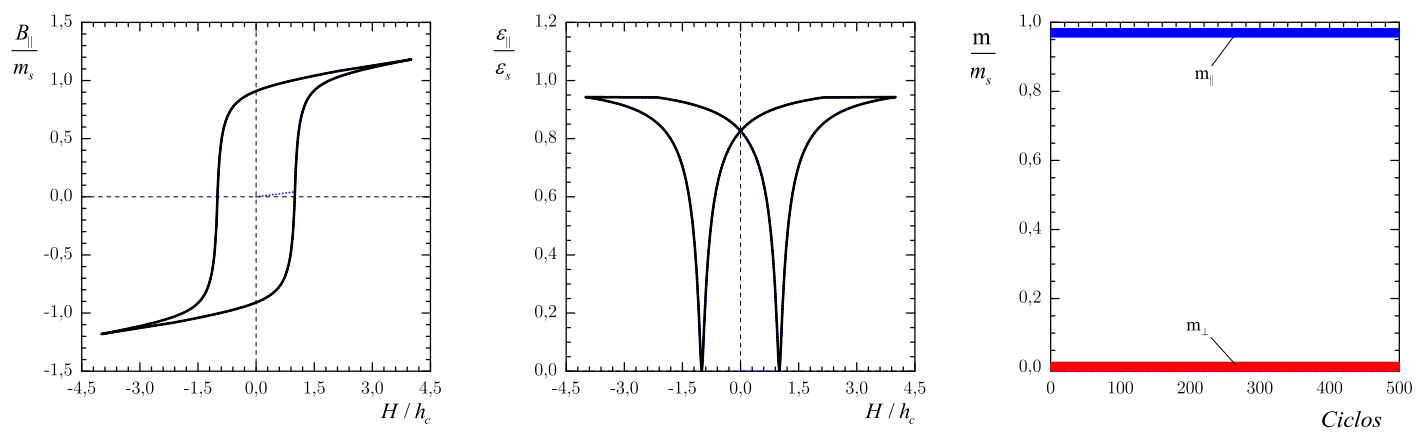

Figura 3.10: Predicciones para especímenes sin cargas mecánicas con exponentes de deformación irreversible $\gamma=1$ y $\gamma=2$ : inducción magnética $\left(B_{\|}\right)$y deformación longitudinal $\left(\varepsilon_{\|}\right)$a lo largo de la dirección del campo magnético aplicado $(H)$, y componentes de magnetización irreversible paralela $\left(\mathrm{m}_{\|}\right)$y perpendicular $\left(\mathrm{m}_{\perp}\right)$.

en función del número de ciclos de carga. Se observa que las respuestas predichas para ambos exponentes de deformación irreversible son consistentes con las observaciones experimentales. Estas predicciones reproducen la respuesta de ciclos de histéresis magnética observados típicamente en aleaciones ferromagnéticas (Clark y Savage, 1983; Kellogg et al., 2002). La magnetización irreversible subyacente permanece alineada con la dirección del campo magnético aplicado, como debe ser en vista de la isotropía inicial del material, y la respuesta se estabiliza inmediatamente después de la primera rampa de carga.

\subsubsection{Estado de carga mecánico uniaxial}

En vistas de las reglas de correspondencia matemática (2.48), es esperable que las predicciones para especímenes bajo tensión mecánica tengan los mismos problemas que las predicciones reportadas en la sección anterior para especímenes ferroeléctricos bajo tensión. Sin embargo, los parámetros materiales empleados en esta sección para 
$\sigma>0$
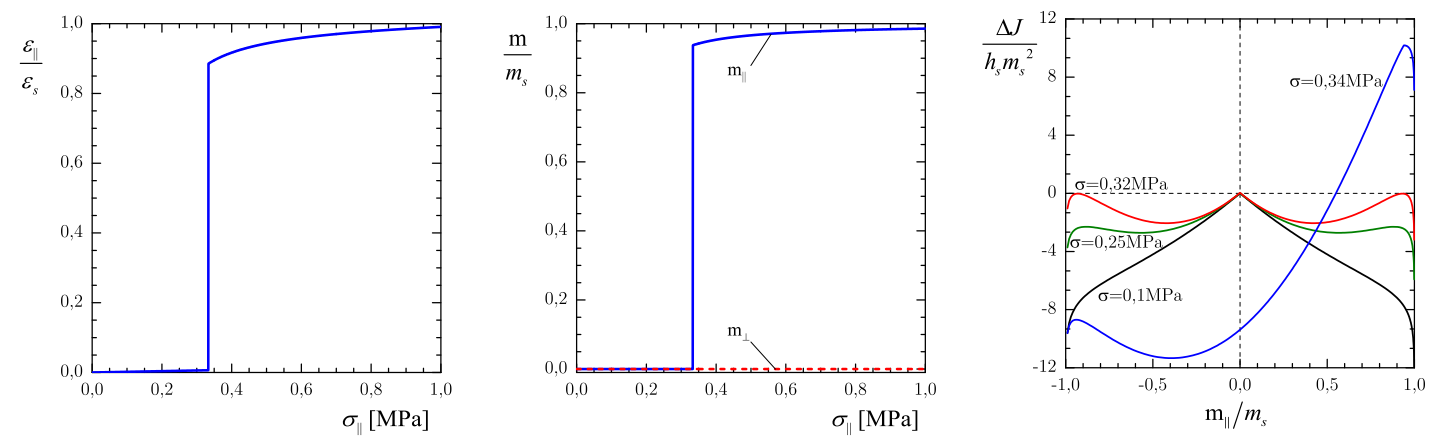

$\sigma<0$
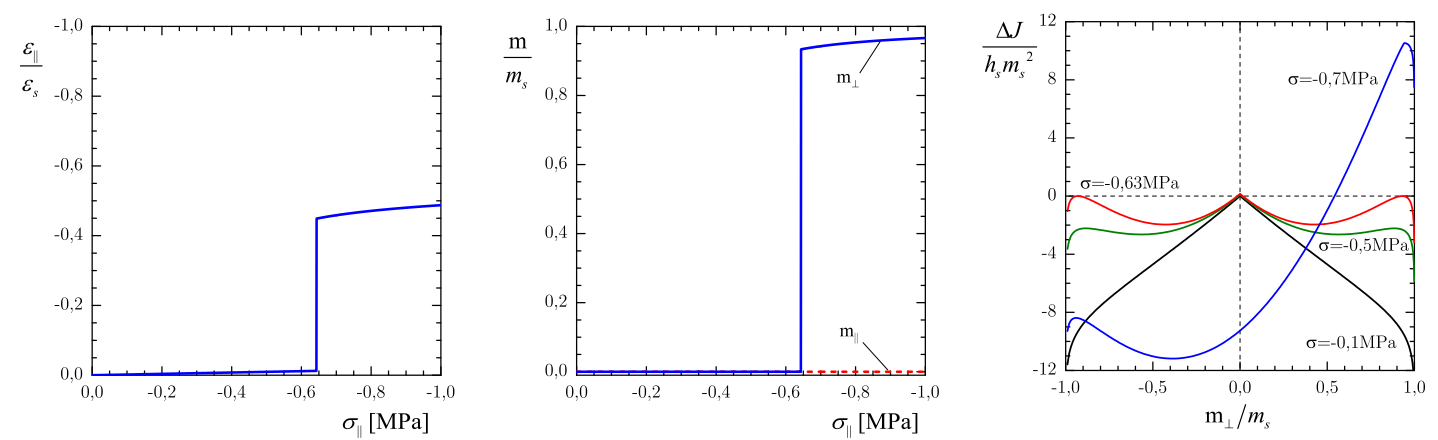

Figura 3.11: Predicciones para especímenes con exponente de deformación irreversible $\gamma=$ 2 sometidos a cargas mecánicas uniaxiales en ausencia de campos magnéticos: deformación longitudinal $\left(\varepsilon_{\|}\right)$a lo largo de la dirección de la tensión aplicada $(\sigma)$, componentes de magnetización irreversible paralela $\left(\mathrm{m}_{\|}\right)$y perpendicular $\left(\mathrm{m}_{\perp}\right)$ y variación de la función objetivo $J$ adimensionalizada.

los especímenes ferromagnéticos dan como resultado problemas aún más serios. En la figura 3.11 se muestran las predicciones para especímenes con $\gamma=2$ sometidos a tensión uniaxial en ausencia de campos magnéticos, es decir, el estado de carga es puramente mecánico. En este caso y teniendo en cuenta que la teoría es incapaz de capturar la ferroelasticidad, se debería predecir una respuesta lineal elástica en la deformación, sin magnetización. Sin embargo, la respuesta predicha muestra un cambio brusco en la deformación axial para ciertos niveles de tensión como consecuencia de una nucleación repentina de magnetización irreversible: en especímenes traccionados, la magnetización irreversible se nuclea en la dirección de la carga, mientras que en especímenes comprimidos se nuclea en la dirección perpendicular. Una vez más, estas predicciones no respetan la física del problema y son consecuencia de la no convexidad de la energía elástica en (2.49). En efecto, si se desprecia el efecto piezomagnético y la contribución de la magnetización irreversible en los tensores flexibilidad y de permitividad magnética, la función $J$ siendo maximizada con respecto a $\mathbf{m}$ en cada 
paso de tiempo es dada por una expresión análoga a (3.1) con $H=0$ :

$$
\begin{aligned}
J \approx \frac{\sigma^{2}}{2 Y}-\sigma \varepsilon_{s}\left(\frac{|\mathbf{m}|}{m_{s}}\right)^{\gamma}\left[1-3\left(\frac{\mathrm{m}_{\|}}{|\mathbf{m}|}\right)^{2}\right] & +h_{s} m_{s}^{2}\left[\ln \left(1-\frac{|\mathbf{m}|}{m_{s}}\right)+\frac{|\mathbf{m}|}{m_{s}}\right]- \\
& -\Delta t h_{c}|\dot{\mathbf{m}}|-\Delta t \frac{h_{0} \dot{m}_{0}}{1+n}\left(\frac{|\dot{\mathbf{m}}|}{\dot{m}_{0}}\right)^{1+n},
\end{aligned}
$$

donde $\dot{\mathbf{m}}=\left(\mathbf{m}-\mathbf{m}_{i}\right) / \Delta t \mathrm{y}|\mathbf{m}|=\sqrt{\mathrm{m}_{\|}^{2}+\mathrm{m}_{\perp}^{2}}$.

La figura 3.11 muestra curvas de $\Delta J=J-\sigma^{2} / 2 Y$ en función de $\mathrm{m}_{\|} \mathrm{y}_{\perp}$ para $\mathbf{m}_{i}=\mathbf{0}$ y varios niveles de tensión $\sigma$. Inicialmente, la función es cóncava y posee un único máximo en $\mathbf{m}=\mathbf{0}$. A medida que el nivel de tensión aumenta, sin embargo, se desarrollan máximos locales para la magnetización en el entorno de $m_{s}$. Para un valor de tensión determinado, estos máximos se vuelven globales y por lo tanto la magnetización óptima salta repentinamente. Este salto ocurre cuando el segundo y cuarto término de (3.4) se cancelan mutuamente. En consecuencia, en tracción $(\sigma>0)$, la magnetización paralela tiene un salto para niveles de tensión $\sim h_{c} m_{s} / \varepsilon_{s}$ mientras que en compresión $(\sigma<0)$ la magnetización perpendicular tiene un salto para niveles de tensión $\sim 2 h_{c} m_{s} / \varepsilon_{s}$. Para los parámetros materiales específicos utilizados aquí, estos niveles de tensión están muy por debajo del megapascal, y pueden ser aún más bajos en presencia de campos magnéticos. Este rango de validez tan acotado hace que esta teoría resulte inadecuada para estudiar procesos magnetomecánicos en sistemas ferromagnéticos. Cabe mencionar que para los especímenes ferroeléctricos considerados en la sección 3.2, la razón $e_{c} p_{s} / \varepsilon_{s}$ es del orden de cien megapascales, por fuera del rango de tensiones considerados en esa sección. Es por esta razón que no se ha presentado este problema allí.

\subsection{Observaciones finales}

El análisis presentado indica que la clase de teorías fenomenológicas utilizada solamente pueden generar predicciones confiables para historias de carga complejas si las tensiones mecánicas permanecen por debajo de cierto límite, que puede ser del orden de algunos megapascales en el caso de cerámicos ferroeléctricos típicos, y muy por debajo de un megapascal en el caso aleaciones ferromagnéticas típicas. Bajo niveles de tensión más elevados, las predicciones se vuelven numéricamente inestables o la solución no es única, dependiendo de la magnitud relativa de los diversos parámetros materiales. Esto es una consecuencia de la dependencia no convexa en la densidad de dipolos permanentes asumida para la energía elástica.

En base a estos resultados, resulta razonable cuestionar la validez de las estimaciones 
presentadas en los trabajos de Miehe y Rosato (2011) y Miehe et al. (2011a). De hecho, es sabido que la implementación mediante el método de los elementos finitos en esos trabajos resulta dificultosa debido a problemas de convergencia en los modelos. Es probable que en esta problemática haya quedado enmascarado el carácter no convexo de la energía libre utilizada, evitando que los investigadores identificaran adecuadamente la problemática. A pesar de esto y considerando que al momento de iniciar este trabajo no existía una teoría superadora, se continúo utilizando estos modelos teniendo siempre presente las limitaciones mencionadas. 


\section{Teoría multiescala para ferrocerámicos multifásicos}

\subsection{Introducción}

La teoría de homogeneización provee un formalismo matemático que permite relacionar de manera rigurosa las propiedades macroscópicas de los materiales con sus propiedades microscópicas y microestructurales subyacentes. A escala macroscópica, la mayor parte de los materiales de uso tecnológico parecen especialmente homogéneos. Sin embargo, a una escala inferior - típicamente del orden de los micrómetroscasi todos ellos exhiben complejas microestructuras conformadas por múltiples fases químicas. Piénsese, por ejemplo, en los aceros duales constituidos por una fase ferrítica y otra martensítica, en los hormigones constituidos por un agregado y un aglomerante, en los elastómeros compuestos constituidos por una matriz polimérica reforzada con partículas duras, o en espumas metálicas constituidas por una matriz metálica y poros. El comportamiento manifestado por estos materiales a escala macroscópica es pues una consecuencia del comportamiento propio de cada una de sus fases constitutivas y de la distribución espacial de las mismas.

Existen múltiples razones por las cuales resulta de sumo interés establecer relaciones entre propiedades macroscópicas y microscópicas. En primer lugar, tales relaciones multiescala permiten cualificar y cuantificar el rol que juegan las diversas características microscópicas en el comportamiento macroscópico e identificar así sistemas materiales con propiedades deseadas u optimizadas. En segundo lugar, el contar con relaciones multiescala facilita la descripción de respuestas complejas difíciles de describir de manera puramente fenomenológica. Este es el caso de las respuestas hereditarias - tales como la elastoplasticidad y la ferroelectricidad - y de las respuestas dependientes de propiedades microestructurales que evolucionan en el tiempo.

En este capítulo se propone una teoría multiescala para el estudio del comportamiento macroscópico de materiales ferrocerámicos multifásicos. Una vez formulada la teoría de medios continuos a nivel micrométrico, se procede a homogeneizar las ecuaciones resultantes para obtener el comportamiento macroscópico del material electroactivo en términos del comportamiento local y de la microestructura. Para resolver luego el problema homogeneizado se propone aquí generalizar la estrategia basada en 'microgeometrías resolubles' de Idiart (2014) para materiales dieléctricos no disipativos al caso de materiales dieléctricos con respuestas hereditarias. Esta estrategia aprovecha 
la solución cuasi analítica de laminados simples, con la cual es posible obtener predicciones para materiales ferrocerámicos multifásicos.

\subsection{Formulación a dos escalas}

En este caso el sistema bajo estudio, representado en la figura 4.1, se idealiza como un sólido heterogéneo ocupando un dominio $\Omega$ y formado por una matriz continua que contiene una dispersión uniforme de inclusiones de segunda fase. La fase de la matriz se identifica con el índice $r=1$ mientras que las inclusiones se identificarán colectivamente con el índice $r=2$. Cada fase ocupa un dominio $\Omega^{(r)} \subset \Omega(r=1,2)$ de modo tal que $\Omega=\Omega^{(1)} \cup \Omega^{(2)}$. Los dominios $\Omega^{(r)}$ pueden describirse mediante un conjunto de funciones características $\chi^{(r)}(\mathbf{x})$, que toman el valor 1 si el vector posición $\mathbf{x}$ está en $\Omega^{(r)}$ y 0 en cualquier otro caso. Nuevamente se restringe la atención a procesos isotérmicos producidos por interacciones electromecánicas cuasiestáticas producidas por un potencial electrostático fijo $\hat{\phi}$ aplicado mediante electrodos en una porción $\partial \Omega_{e}$ del borde del cuerpo $\partial \Omega$, mediante un desplazamiento superficial $\hat{\mathbf{u}}$ aplicado en una porción $\partial \Omega_{u}$ del borde del cuerpo y por una tracción $\hat{\mathbf{t}}$ aplicada en el complemento del borde del cuerpo.

En particular, se centra ahora la atención en sistemas materiales para los cuales el tamaño característico de las heterogeneidades microestructurales — partículas o cavidades - es mucho menor que el tamaño característico del material compuesto en estudio. Esto permite utilizar los conceptos de la teoría de homogeneización para definir la respuesta macroscópica u homogeneizada del material compuesto como la relación entre campos conjugados promediados en un "elemento de volumen representativo" (EVR) $\Xi$ que contiene un número suficiente de heterogeneidades para que la respuesta macroscópica sea efectivamente independiente de las condiciones de borde predominantes (Germain et al., 1983; Hill, 1963). Las ecuaciones de campo del espécimen están dadas por las expresiones (2.22)-(2.26) con un potencial incremental $w$ dependiente de $\mathbf{x}$. En virtud de su estructura matemática, se pueden utilizar resultados convencionales de homogeneización no lineal (por ejemplo, Ponte Castañeda y Suquet, 1998) para obtener una formulación a dos escalas. En consecuencia, las ecuaciones de campo del problema macroscópico pueden escribirse como

$$
\begin{aligned}
& \nabla \cdot \overline{\mathbf{D}}=0 \quad \text { y } \quad \overline{\mathbf{E}}=-\nabla \bar{\phi} \quad \text { en } \mathbb{R}^{3}, \\
& \nabla \cdot \overline{\boldsymbol{\sigma}}=0 \quad \text { y } \quad \overline{\boldsymbol{\varepsilon}}=\nabla \otimes_{s} \overline{\mathbf{u}} \quad \text { en } \Omega,
\end{aligned}
$$




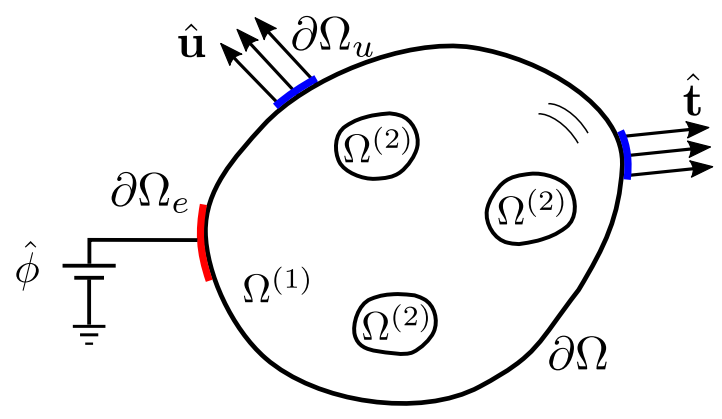

Figura 4.1: Dominio y condiciones de borde para un ferrocerámico con inclusiones.

con

$$
\overline{\mathbf{D}}= \begin{cases}\epsilon_{0} \overline{\mathbf{E}} & \text { en } \mathbb{R}^{3} \backslash \Omega \\ \epsilon_{0} \overline{\mathbf{E}}+\overline{\mathbf{P}} & \text { en } \Omega\end{cases}
$$

y las condiciones de borde

$$
\begin{array}{lllll}
\bar{\phi}=\hat{\phi} & \text { en } \partial \Omega_{e} & \text { y } & \llbracket \overline{\mathbf{D}} \rrbracket \cdot \mathbf{n}=0 & \text { en } \partial \Omega \backslash \partial \Omega_{e} \\
\overline{\mathbf{u}}=\hat{\mathbf{u}} & \text { en } \partial \Omega_{u} & \text { y } & \overline{\boldsymbol{\sigma}} \mathbf{n}=\hat{\mathbf{t}} & \text { en } \partial \Omega \backslash \partial \Omega_{u}
\end{array}
$$

En las expresiones anteriores, el símbolo - representa el valor macroscópico, obtenido a través del promedio en $\Xi$. Adicionalmente, el potencial electrostático debe anularse en el infinito, es decir, $\phi \rightarrow 0$ cuando $|\mathbf{x}| \rightarrow \infty$.

Por otro lado, las ecuaciones de campo microscópicas son

$$
\begin{aligned}
& \mathbf{E}=-\nabla \phi, \quad \nabla \cdot \mathbf{D}=0 \quad \text { en } \Xi, \\
& \nabla \boldsymbol{\sigma}=\mathbf{0}, \quad \boldsymbol{\varepsilon}=\nabla \otimes_{s} \mathbf{u} \quad \text { en } \Xi \text {, } \\
& \mathbf{D}=\frac{\partial w}{\partial \mathbf{E}}\left(\mathbf{x}, \boldsymbol{\sigma}, \mathbf{E} ; \mathrm{p}_{i}\right), \quad \boldsymbol{\varepsilon}=\frac{\partial w}{\partial \boldsymbol{\sigma}}\left(\mathbf{x}, \boldsymbol{\sigma}, \mathbf{E} ; \mathrm{p}_{i}\right) \quad \text { en } \Xi, \\
& \phi=-\overline{\mathbf{E}} \cdot \mathbf{x}, \quad \mathbf{t}=\overline{\boldsymbol{\sigma}} \mathbf{n} \quad \text { en } \partial \Xi,
\end{aligned}
$$

donde el potencial incremental se define ahora teniendo en cuenta las funciones características $\chi^{(r)}$ dentro del EVR de la siguiente manera

$$
w\left(\mathbf{x}, \boldsymbol{\sigma}, \mathbf{E} ; \mathrm{p}_{i}\right)=\sum_{r=1}^{2} \chi^{(r)}(\mathbf{x}) w^{(r)}\left(\boldsymbol{\sigma}, \mathbf{E} ; \mathrm{p}_{i}\right)
$$

con

$$
w^{(r)}\left(\boldsymbol{\sigma}, \mathbf{E} ; \mathrm{p}_{i}\right)=\sup _{\mathrm{p}} J^{(r)}\left(\boldsymbol{\sigma}, \mathbf{E}, \mathrm{p} ; \mathrm{p}_{i}\right)
$$




$$
J^{(r)}\left(\boldsymbol{\sigma}, \mathbf{E}, \mathrm{p} ; \mathrm{p}_{i}\right)=\psi^{(r)}(\boldsymbol{\sigma}, \mathbf{E}, \mathrm{p})-\Delta t \varphi^{(r)}\left(\frac{\mathrm{p}-\mathrm{p}_{i}}{\Delta t}\right)
$$

A través de las superficies internas de discontinuidad, los diversos campos deben además satisfacer las condiciones de salto

$$
\llbracket \phi \rrbracket=0, \quad \llbracket \mathbf{D} \cdot \mathbf{n} \rrbracket=0, \quad \llbracket \mathbf{u} \rrbracket=0, \quad \llbracket \boldsymbol{\sigma} \mathbf{n} \rrbracket=\mathbf{0},
$$

donde $\mathbf{n}$ denota el vector normal a la superficie de discontinuidad.

Las ecuaciones de campo, relaciones constitutivas y condiciones de borde (4.6)(4.9), junto con las condiciones de salto (4.13) y condiciones iniciales apropiadas, definen completamente la respuesta electromecánica microscópica del sistema bajo consideración.

La respuesta macroscópica puede escribirse en términos de un potencial incremental efectivo. Para ello se tiene en cuenta que el sistema (4.6)-(4.9) son las ecuaciones de Euler-Lagrange del problema variacional (Miehe, 2002)

$$
\widetilde{w}\left(\overline{\boldsymbol{\sigma}}, \overline{\mathbf{E}} ; \mathrm{p}_{i}\right)=\min _{\mathbf{E} \in \mathcal{K}(\overline{\mathbf{E}})} \min _{\boldsymbol{\sigma} \in \mathcal{S}(\overline{\boldsymbol{\sigma}})}\left\langle w\left(\mathbf{x}, \boldsymbol{\sigma}, \mathbf{E} ; \mathrm{p}_{i}\right)\right\rangle
$$

donde $\widetilde{w}$ es un potencial incremental efectivo, $\langle\cdot\rangle$ denota el promedio volumétrico en $\Xi$, y $\mathcal{K}(\overline{\mathbf{E}})$ y $\mathcal{S}(\overline{\boldsymbol{\sigma}})$ denotan el conjunto de campos eléctricos admisibles $\mathbf{E}$ y el conjunto de campos estáticamente admisibles $\boldsymbol{\sigma}$, respectivamente, definidos como

$$
\begin{aligned}
& \mathcal{S}(\overline{\boldsymbol{\sigma}})=\{\boldsymbol{\sigma}, \nabla \cdot \boldsymbol{\sigma}=0 \text { en } \Omega,\langle\boldsymbol{\sigma}\rangle=\overline{\boldsymbol{\sigma}}\} \\
& \mathcal{K}(\overline{\mathbf{E}})=\{\mathbf{E}, \mathbf{E}=-\nabla \phi \text { en } \Omega,\langle\mathbf{E}\rangle=\overline{\mathbf{E}}\} .
\end{aligned}
$$

Además, del lema de Hill se tiene que

$$
\overline{\mathbf{D}}=\frac{\partial \widetilde{w}}{\partial \overline{\mathbf{E}}}\left(\overline{\boldsymbol{\sigma}}, \overline{\mathbf{E}} ; \mathrm{p}_{i}\right) \quad \text { y } \quad \overline{\boldsymbol{\varepsilon}}=\frac{\partial \widetilde{w}}{\partial \overline{\boldsymbol{\sigma}}}\left(\overline{\boldsymbol{\sigma}}, \overline{\mathbf{E}} ; \mathrm{p}_{i}\right)
$$

donde las magnitudes macroscópicas son tales que $\overline{\mathbf{D}}=\langle\mathbf{D}\rangle, \overline{\mathbf{E}}=\langle\mathbf{E}\rangle, \overline{\boldsymbol{\sigma}}=\langle\boldsymbol{\sigma}\rangle$ y $\bar{\varepsilon}=\langle\varepsilon\rangle$. Las expresiones en (4.16) constituyen la respuesta instantánea macroscópica del sólido, que queda caracterizada completamente mediante el potencial efectivo $\widetilde{w}$ y relaciona los promedios del desplazamiento eléctrico y la deformación con los promedios de la tensión y el campo eléctrico en $\Xi$.

La respuesta macroscópica completa se obtiene resolviendo el problema de minimización (4.14) para un dado $\mathrm{p}_{i}$ en cada paso de tiempo e integrando (4.16) en el tiempo. Cabe destacarse que, a pesar de las apariencias, el potencial efectivo no se 
corresponde estrictamente a un material compuesto bifásico, sino más bien a un material compuesto con un infinito número de fases - este hecho fue inicialmente observado por Lahellec y Suquet (2007) en el contexto de viscoelasticidad. Esto se debe a que el campo $\mathrm{p}_{i}$ es, en general, heterogéneo incluso en el interior de cada fase constitutiva. A priori, esto entra en conflicto con uno de los propósitos prácticos de los procedimientos de homogeneización, que es generar una descripción simple de la respuesta macroscópica en función de un número reducido de variables macroscópicas. Sin embargo, la clase especial de sistemas materiales considerados en la siguiente sección son particulares precisamente en que los campos microscópicos, incluyendo a $\mathrm{p}_{i}$, tienen una distribución discreta y, consecuentemente, la respuesta macroscópica depende de un número finito de variables macroscópicas.

\subsection{Modelo de laminados secuenciales para compuestos particulados}

Debido a la compleja geometría de las microestructuras típicas, el cálculo del potencial macroscópico $\widetilde{w}$ correspondiente a un determinado potencial microscópico $w$ representativo de un material real resulta extremadamente complicado. La estrategia basada en "microgeometrías resolubles" consiste en identificar microgeometrías idealizadas que, por un lado, sean capaces de representar las características geométricas esenciales de la microestructura en estudio y, por otro lado, sean lo suficientemente simples como para permitir el cálculo exacto del potencial $\widetilde{w}$ de manera semianalítica. Este tipo de estrategia fue inicialmente utilizada por Maxwell (1873) en conductores bifásicos para estimar su resistividad óhmica, y se ha utilizado mucho desde entonces para estimar propiedades lineales de materiales compuestos — véase, por ejemplo, Milton (2002). En el contexto de materiales no lineales, su utilización ha sido muy limitada debido a las dificultades adicionales inherentes a los problemas matemáticos no lineales. Recientemente, Idiart (2014) construyó microgeometrías cuyo potencial, semejante al presentado en la sección anterior, puede calcularse en forma exacta. Estas microgeometrías - que ya han sido utilizadas en otros contextos - se denominan laminados secuenciales.

Un laminado secuencial es una construcción iterativa que se obtiene iterando laminaciones simples, obtenidas a partir de procedimientos de laminación de orden inferior, con otros materiales laminados o directamente con las fases homogéneas que conforman el material compuesto. El rango del laminado se refiere al número de operaciones de laminación requeridas para alcanzar el laminado secuencial final. En este caso, se centra la atención en laminados bifásicos compuestos por una matriz continua conteniendo una dispersión aleatoria de inclusiones, por lo que se utiliza 
a)

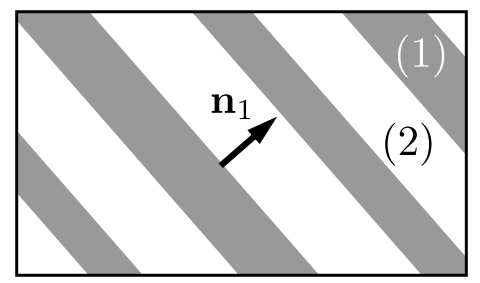

b)

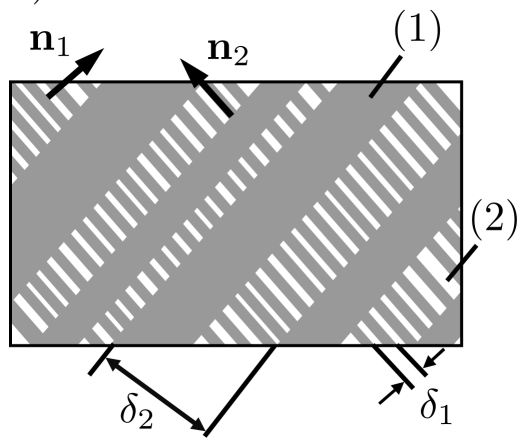

Figura 4.2: Laminados bifásicos: a) laminado de rango 1 , b) laminado rango $2\left(\delta_{2}>>\delta_{1}\right)$.

la secuencia de Idiart $(2008,2014)$. En esta secuencia, un laminado de rango 1 corresponde a un laminado simple con una dirección de laminación $\mathbf{n}_{1}$, con fases 1 y 2 en proporciones $\left(1-f_{1}\right)$ y $f_{1}$, como se observa en la figura $\left.4.2 \mathrm{a}\right)$. Un laminado de rango 2 se construye combinando un laminado de rango 1 con la matriz del material $r=1$, a lo largo de la dirección de laminación $\mathbf{n}_{2}$, en proporciones $f_{2}$ y $1-f_{2}$, asumiendo que la escala de longitud del laminado embebido es mucho menor que el laminado final, como se observa esquemáticamente en la figura 4.2b). Un laminado de rango $M$ se obtiene repitiendo este proceso $M$ veces, siempre combinando un laminado de rango $m$ con matriz del material $r=1$, en proporciones $f_{m}$ y $\left(1-f_{m}\right)$, respectivamente, con dirección de laminación $\mathbf{n}_{m}$. El potencial incremental efectivo del laminado de rango $M$ se obtiene generalizando el resultado de Idiart (2014) para ferroelectricidad de sólidos rígidos, donde se hace uso de la solución conocida para laminados simples y el teorema de la homogeneización iterativa — véase Idiart (2008) para una derivación en el contexto de viscoplasticidad-

$$
\begin{aligned}
\widetilde{w}\left(\overline{\boldsymbol{\sigma}}, \overline{\mathbf{E}} ; \mathrm{p}_{i}\right)=\min _{\substack{a_{i} \in \mathbb{R} \\
i=1, \ldots, M}} \operatorname{mín}_{\substack{\boldsymbol{\omega}_{i} \in \mathcal{S}\left(\mathbf{n}_{i}\right) \\
i=1, \ldots, M}}\left\{c^{(2)} w^{(2)}\left(\overline{\boldsymbol{\sigma}}^{(2)}, \overline{\mathbf{E}}^{(2)} ; \mathrm{p}_{i}^{(2)}\right)+\right. \\
\left.+c^{(1)} \sum_{i=1}^{M} \alpha_{i} w^{(1)}\left(\overline{\boldsymbol{\sigma}}_{i}^{(1)}, \overline{\mathbf{E}}_{i}^{(1)} ; \mathrm{p}_{n, i}^{(1)}\right)\right\},
\end{aligned}
$$

donde $\mathcal{S}(\mathbf{n})=\{\boldsymbol{\omega}$ tal que $\boldsymbol{\omega n}=\mathbf{0}\}, \mathrm{y}$

$$
c^{(2)}=1-c^{(1)}=\prod_{i=1}^{M} f_{i} \quad \text { y } \quad \alpha_{i}=\frac{\left(1-f_{i}\right)}{f_{i}} \frac{\prod_{j=i}^{M} f_{j}}{1-\prod_{j=1}^{M} f_{j}}
$$

son variables microestructurales que representan, respectivamente, la fracción volumétrica total de cada material $r$ - tales que $c^{(1)}+c^{(2)}=1-$ y la fracción de material de la matriz agregado en la iésima laminación - de modo tal que $\alpha_{i}>0$ y $\sum_{i=1}^{M} \alpha_{i}=1$. A su vez, las intensidades de campo eléctrico y los tensores de tensiones 
están dados por

$$
\begin{array}{lc}
\overline{\mathbf{E}}_{i}^{(1)}=\overline{\mathbf{E}}+a_{i} \mathbf{n}_{i}-\sum_{j=i}^{M}\left(1-f_{j}\right) a_{j} \mathbf{n}_{j}, & i=1, \ldots, M, \\
\overline{\boldsymbol{\sigma}}_{i}^{(1)}=\overline{\boldsymbol{\sigma}}+\boldsymbol{\omega}_{i}-\sum_{j=i}^{M}\left(1-f_{j}\right) \boldsymbol{\omega}_{j}, & i=1, \ldots, M, \\
\overline{\mathbf{E}}^{(2)}=\overline{\mathbf{E}}-\sum_{j=1}^{M}\left(1-f_{j}\right) a_{j} \mathbf{n}_{j}, & \overline{\boldsymbol{\sigma}}^{(2)}=\overline{\boldsymbol{\sigma}}-\sum_{j=1}^{M}\left(1-f_{j}\right) \boldsymbol{\omega}_{j} .
\end{array}
$$

Una observación clave para derivar la expresión (4.17) iterativamente es que la representación variacional (4.14) es válida para respuestas microscópicas de la forma (4.8) con cualquier potencial incremental — no necesariamente de la forma (2.20) - y por lo tanto incluye la respuesta macroscópica (4.16). Debe notarse que el potencial incremental $w^{(2)}$ se evalúa para un único valor del campo eléctrico y la polarización irreversible, mientras que el potencial $w^{(1)}$ se evalúa en $M$ valores distintos. Esto significa que los campos son uniformes dentro de la fase de inclusión $r=2 \mathrm{y}$ discretamente no uniformes dentro de la fase de la matriz $r=1$.

El potencial incremental efectivo (4.17) depende de las fracciones volumétricas totales $c^{(r)}=\left\langle\chi^{(r)}(\mathbf{x})\right\rangle$ de cada fase $r$ y de correlaciones microestructurales de orden superior a través del conjunto de cantidades microestructurales $\left\{\left(f_{i}, \mathbf{n}_{i}\right), i=1, \ldots, M\right\}$. Por lo tanto, para poder utilizar estas construcciones para modelar materiales compuestos, las cantidades $\left(f_{i}, \mathbf{n}_{i}\right)$ deben ser expresadas en términos de correlaciones multipunto de las microgeometrías. Sin embargo, en general esto no es factible. Una estrategia consiste entonces en identificar subclases de construcciones de laminados secuenciales para las cuales la dependencia del potencial total respecto a correlaciones de orden superior pueda hacerse explícita (Idiart, 2008; Idiart y Ponte Castañeda, 2013). Otra estrategia consiste en identificar subclases para las cuales el potencial macroscópico presente características deseables, tales como un cierto grupo de simetría (Danas et al., 2008; Hariton y DeBotton, 2003). En cualquier caso, resulta conveniente relacionar las magnitudes $\left(f_{i}, \mathbf{n}_{i}\right)$ con las denominadas "medidas $H$ " de la microgeometría $\mu^{(r s)}(\mathbf{n})$ $(r, s=1,2)$. Estas medidas son objetos geométricos que dependen de funciones de correlación de dos puntos $\left\langle\chi^{(r)}(\mathbf{x}) \chi^{(s)}(\mathbf{x}-\mathbf{y})\right\rangle$ de la microestructura y cuantifican, en el espacio de fases, la falta de compacidad de secuencias de convergencia débil de las funciones características $\left[\chi^{(r)}(\mathbf{x})-c^{(r)}\right]$ y proveen una caracterización parcial de las oscilaciones microestructurales a lo largo de distintas direcciones en el espacio físico (Tartar, 1990). Para microestructuras aleatorias que son estadísticamente uniformes y ergódicas, las medidas H pueden escribirse como — véase, por ejemplo, Smyshlyaev 
y Willis (1998)-

$$
\mu^{(r s)}(\mathbf{n})=-\frac{1}{8 \pi^{2}} \int_{\mathbb{R}^{3}} \delta^{\prime \prime}(\mathbf{n} \cdot \mathbf{x})\left(\left\langle\chi^{(r)}(\mathbf{y}) \chi^{(s)}(\mathbf{y}-\mathbf{x})\right\rangle-c^{(r)} c^{(s)}\right) \mathrm{d} \mathbf{x}
$$

y para la clase particular de microgeometrías laminadas secuencialmente consideradas aquí toman la forma

$$
\mu^{(r s)}(\mathbf{n})=c^{(r)}\left(\delta_{r s}-c^{(s)}\right) \bar{\nu}_{M}(\mathbf{n})
$$

donde $\bar{\nu}_{M}(\mathbf{n})$ es la medida $H$ reducida del laminado secuencial y está dada por — véase, por ejemplo, Idiart y Ponte Castañeda (2013)—

$$
\bar{\nu}_{M}(\mathbf{n})=\sum_{i=1}^{M} \nu_{i} \delta\left(\mathbf{n}-\mathbf{n}_{i}\right), \quad \text { con } \quad \nu_{i}=\frac{1}{c^{(1)}} \frac{1-f_{i}}{f_{i}} \prod_{j=1}^{i} f_{j},
$$

donde $\delta(\cdot)$ denota la función delta de Dirac evaluada en vectores, y las cantidades $\nu_{i}$ satisfacen las condiciones

$$
\nu_{i} \geq 0 \quad \text { and } \quad \sum_{i=1}^{M} \nu_{i}=1
$$

Las relaciones $(4.23)_{2}$ pueden invertirse para expresar los $f_{i}$ en función de los $\nu_{i}$ como

$$
f_{i}=\frac{1-c^{(1)}\left(1+\nu_{i}-\sum_{j=i}^{M} \nu_{j}\right)}{1-c^{(1)}\left(1-\sum_{j=i}^{M} \nu_{j}\right)} .
$$

Reemplazando los $f_{i}$ en las expresiones (4.17)-(4.20) en favor de los $\nu_{i}$, se obtiene una expresión alternativa para el potencial incremental efectivo $\widetilde{w}$ que depende de la microgeometría subyacente a través de la fracción volumétrica total $c^{(r)}$ de cada material $r$ y del conjunto $\left\{\left(\nu_{i}, \mathbf{n}_{i}\right)\right\}$ sometido a las restricciones (4.24).

Cabe destacar que la medida $H$ reducida $\bar{\nu}_{M}(\mathbf{n})$ queda determinada completamente mediante este conjunto, pero el conjunto no puede determinarse completamente a partir de la medida $H$ reducida $\bar{\nu}_{M}$. Esto se debe en primer lugar a que no existe una relación biyectiva entre la función $\bar{\nu}_{M}(\mathbf{n})$ y los $\nu_{i}$ cuando la secuencia de laminación involucra direcciones de laminación repetidas, y que además la función $\bar{\nu}_{M}$ es insensible al orden de los elementos del conjunto. El potencial macroscópico $\widetilde{w}_{M}$ entonces depende, en general, de información de la microestructura más allá de las fracciones volumétricas y la medida $H$. De todos modos, las cantidades $\nu_{i}$ son un medio conveniente para identificar secuencias de laminación adecuadas, como se verá 
más adelante.

El hecho de que estos sistemas materiales representan características esenciales de compuestos bifásicos con microestructuras particuladas se apoya en comparaciones con simulaciones de campo completo y estimaciones aproximadas presentadas en diversos trabajos en compuestos puramente energéticos/disipativos (Danas et al., 2008; Hariton y DeBotton, 2003; Idiart, 2008; Idiart et al., 2006). Además, cuando ambas fases son rígidos dieléctricos ideales, el potencial efectivo (4.17) coincide exactamente con la aproximación de Maxwell-Garnett — también conocida como la aproximación de Claussius-Mossotti - para dieléctricos lineales bifásicos con medida $H$ (4.22). Por último, si se utiliza la aproximación de polarización uniforme (2.45), el potencial efectivo (4.17) se reduce al presentado por Spinelli y Lopez-Pamies (2014).

Si los potenciales incrementales $w^{(r)}$ son convexos, el cálculo del potencial efectivo (4.17) para determinadas variables macroscópicas $\overline{\boldsymbol{\sigma}}, \overline{\mathbf{E}}$ y polarización irreversible $\mathrm{p}_{i}^{(r)}$ requiere la solución de un problema de optimización convexo con respecto a los $a_{i} \mathrm{y}$ $\boldsymbol{\omega}_{i}$. Las variables conjugadas correspondientes $\overline{\mathbf{D}}$ y $\bar{\varepsilon}$ pueden luego ser calculadas por derivación, y en vistas de las condiciones de minimización, se puede demostrar que

$$
\begin{array}{r}
\overline{\mathbf{D}}=\frac{\partial \widetilde{w}}{\partial \overline{\mathbf{E}}}\left(\overline{\boldsymbol{\sigma}}, \overline{\mathbf{E}} ; \mathrm{p}_{i}\right)=c^{(1)} \sum_{i=1}^{M} \alpha_{i} \frac{\partial w^{(1)}}{\partial \mathbf{E}}\left(\overline{\boldsymbol{\sigma}}_{i}^{(1)}, \overline{\mathbf{E}}_{i}^{(1)} ; \mathrm{p}_{n, i}^{(1)}\right)+ \\
+c^{(2)} \frac{\partial w^{(2)}}{\partial \mathbf{E}}\left(\overline{\boldsymbol{\sigma}}^{(2)}, \overline{\mathbf{E}}^{(2)} ; \mathrm{p}_{i}^{(2)}\right), \\
\overline{\boldsymbol{\varepsilon}}=\frac{\partial \widetilde{w}}{\partial \overline{\boldsymbol{\sigma}}}\left(\overline{\boldsymbol{\sigma}}, \overline{\mathbf{E}} ; \mathrm{p}_{i}\right)=c^{(1)} \sum_{i=1}^{M} \alpha_{i} \frac{\partial w^{(1)}}{\partial \boldsymbol{\sigma}}\left(\overline{\boldsymbol{\sigma}}_{i}^{(1)}, \overline{\mathbf{E}}_{i}^{(1)} ; \mathrm{p}_{n, i}^{(1)}\right)+ \\
+c^{(2)} \frac{\partial w^{(2)}}{\partial \boldsymbol{\sigma}}\left(\overline{\boldsymbol{\sigma}}^{(2)}, \overline{\mathbf{E}}^{(2)} ; \mathrm{p}_{i}^{(2)}\right),
\end{array}
$$

donde los campos microscópicos son evaluados en la solución óptima. Adicionalmente, se puede calcular la estadística de los campos microscópicos a partir de las expresiones (4.19)-(4.20). En particular, el primer y segundo momento de la distribución de tensiones dentro de cada fase están dados por

$$
\begin{aligned}
& \langle\boldsymbol{\sigma}\rangle^{(1)}=\sum_{i=1}^{M} \alpha_{i} \overline{\boldsymbol{\sigma}}_{i}^{(1)} \quad \mathrm{y} \quad\langle\boldsymbol{\sigma}\rangle^{(2)}=\overline{\boldsymbol{\sigma}}^{(2)} \\
& \langle\boldsymbol{\sigma} \otimes \boldsymbol{\sigma}\rangle^{(1)}=\sum_{i=1}^{M} \alpha_{i} \overline{\boldsymbol{\sigma}}_{i}^{(1)} \otimes \overline{\boldsymbol{\sigma}}_{i}^{(1)} \quad \mathrm{y} \quad\langle\boldsymbol{\sigma} \otimes \boldsymbol{\sigma}\rangle^{(2)}=\overline{\boldsymbol{\sigma}}^{(2)} \otimes \overline{\boldsymbol{\sigma}}^{(2)},
\end{aligned}
$$

mientras que el promedio volumétrico y la desviación estándar del campo eléctrico 
en la matriz pueden calcularse mediante

$$
\langle\mathbf{E}\rangle^{(1)}=\sum_{i=1}^{M} \alpha_{i} \overline{\mathbf{E}}_{i}^{(1)} \quad \mathrm{y} \quad S D^{(1)}(E)=\left[\sum_{i=1}^{M} \alpha_{i}\left|\overline{\mathbf{E}}_{i}^{(1)}\right|^{2}-\left|\langle\mathbf{E}\rangle^{(1)}\right|^{2}\right]^{1 / 2},
$$

y la estadística del campo de polarización puede calcularse de manera análoga

$$
\langle\mathrm{p}\rangle^{(1)}=\sum_{i=1}^{M} \alpha_{i} \overline{\mathrm{p}}_{i}^{(1)} \quad \mathrm{y} \quad S D^{(1)}(\mathrm{p})=\left[\sum_{i=1}^{M} \alpha_{i}\left|\overline{\mathrm{p}}_{i}^{(1)}\right|^{2}-\left|\langle\mathrm{p}\rangle^{(1)}\right|^{2}\right]^{1 / 2} .
$$

En este trabajo se centra la atención en sistemas materiales que poseen simetría isótropa global. Motivado por los trabajos de Hariton y DeBotton (2003) e Idiart et al. (2006) — véase también Danas et al. (2008)—, se adopta la siguiente secuencia de laminación:

$$
\begin{gathered}
\nu_{i}=\frac{1}{M}, \\
\mathbf{n}_{i}=\sin \alpha_{i} \sin \beta_{i} \mathbf{e}_{1}+\cos \alpha_{i} \sin \beta_{i} \mathbf{e}_{2}+\cos \beta_{i} \mathbf{e}_{3}, \quad i=1, \ldots, M,
\end{gathered}
$$

donde

$$
\beta_{j+k M_{\eta}}=\arccos h_{j}, \quad h_{j}=2 \frac{j-1}{M_{\eta}-1}-1, \quad \begin{aligned}
j & =1, \ldots, M_{\eta}, \\
k & =0, \ldots, \eta-1,
\end{aligned}
$$

$\mathrm{y}$

$$
\begin{gathered}
\alpha_{1}=\alpha_{M_{\eta}}=0, \\
\alpha_{j+k M_{\eta}}=\left(\alpha_{j-1}+\frac{3,6}{\sqrt{M_{\eta}}} \frac{1}{\sqrt{1-h_{j}^{2}}}\right) \bmod 2 \pi, \quad \begin{array}{l}
j=2, \ldots, M_{\eta}-1, \\
k=0, \ldots, \eta-1 .
\end{array}
\end{gathered}
$$

En estas expresiones, los ángulos $\alpha_{i}$ y $\beta_{i}$ determinan la iésima dirección de laminación relativa a la base de referencia $\left\{\mathbf{e}_{i}\right\}$, y los parámetros $\eta$ y $M_{\eta}$ son enteros tales que el rango del laminado es $M=\eta M_{\eta}$. El conjunto de ángulos (4.34)-(4.36) corresponde a las $M_{\eta}$ direcciones de laminación (4.33) distribuidas uniformemente en una esfera unitaria (Saff y Kuijlaars, 1997), con $\eta$ laminaciones para cada dirección. A su vez, las cantidades $\nu_{i}$ se consideran todas iguales y satisfacen las restricciones (4.24). Esta es una secuencia para la cual la medida $H$ se vuelve isótropa a medida que $M_{\eta} \rightarrow \infty$, para cualquier número de rondas $\eta$. Sin embargo, las microgeometrías resultantes en ese límite no son isótropas. Para poder generar sistemas materiales que tengan respuestas macroscópicas isótropas, también debería tomarse el segundo límite $\eta \rightarrow \infty$ - una discusión más detallada puede encontrarse en Idiart y Ponte Castañeda (2013). Se ha verificado numéricamente que, para los materiales considerados en este trabajo, la respuesta macroscópica se vuelve progresivamente menos sensible a la orientación de los campos aplicados a medida que el parámetro $M_{\eta}$ y $\eta$ aumentan. 
Particularmente, los valores $\eta=5$ y $M_{\eta}=50$, que corresponden a laminados secuenciales de rango $M=250$, han demostrado ser adecuados para los propósitos de este trabajo y serán utilizados para generar las predicciones del próximo capítulo. 


\section{Aplicación a ferrocerámicos porosos y con inclusiones}

\subsection{Resultados para sistemas materiales representativos}

La teoría multiescala desarrollada en el capítulo 4 a partir del modelo material presentado en el capítulo 2 se utiliza aquí para analizar la influencia de inclusiones de segunda fase en el comportamiento de ferrocerámicos policristalinos. En particular, se consideran casos extremos de inclusiones de partículas metálicas y microcavidades. En ambos casos, la matriz ferroeléctrica es caracterizada con potenciales de la forma (2.31)-(2.37). Por otro lado, las partículas metálicas se consideran conductores perfectos elásticos caracterizados por potenciales de la forma (2.27)-(2.29), y se asume que las microcavidades están vacías. En la tabla 5.1 se muestran los valores numéricos adoptados para los parámetros materiales. Los valores para la matriz ferroeléctrica reproducen aproximadamente el comportamiento sensible a la tasa de PZT policristalino a bajas frecuencias (Miehe y Rosato, 2011; Zhou et al., 2001); los valores para las partículas metálicas corresponden a platino. Los resultados presentados consideran la definición de los tensores del material ferrocerámico presentados en (2.40). Se asume que la segunda fase está distribuida de manera isótropa y aleatoriamente, por lo que se adopta la secuencia de laminación $\left\{\nu_{i}, \mathbf{n}_{i}\right\}$ presentada en (4.32)-(4.36) con rango $M=250$. Cabe destacar que, como se observa en los resultados presentados a continuación, las predicciones obtenidas percolan para una concentración de inclusiones de $c^{(2)}=1$, por lo que se espera que las conclusiones derivadas de los mismos sean relevantes para materiales compuestos donde la segunda fase es polidispersa.

Para cada valor de los campos $\overline{\boldsymbol{\sigma}}$ y $\overline{\mathbf{E}}$ aplicados, el problema de minimización con respecto a los $a_{i}$ y $\boldsymbol{\omega}_{i}$ de (4.17) es resuelto mediante un método de optimización de cuasi Newton para funciones suaves y convexas, mientras que las polarizaciones irreversibles en los potenciales $w^{(r)}$ en (4.11) se obtienen utilizando algoritmos de búsqueda directa compleja para funciones convexas no suaves. Teniendo en cuenta que los coeficientes $a_{i}$ y $\boldsymbol{\omega}_{i}$ determinan el valor de los campos locales en las fases, deberán considerarse las restricciones correspondientes al comportamiento del tipo de inclusión analizada. Para partículas metálicas, la minimización con respecto a los $a_{i}$ se realiza considerando la restricción vectorial lineal $\overline{\mathbf{E}}^{(2)}=\mathbf{0}$ con $\overline{\mathbf{E}}^{(2)}$ dado por $(4.20)_{1}$, mientras que para microcavidades, la minimización con respecto a los $\boldsymbol{\omega}_{i}$ se realiza considerando la restricción tensorial lineal $\overline{\boldsymbol{\sigma}}^{(2)}=\mathbf{0}$ con $\overline{\boldsymbol{\sigma}}^{(2)}$ dado por $(4.20)_{2}$. El paso de tiempo empleado en los cálculos es $\Delta t=10^{-3} \mathrm{~s}$. 
Tabla 5.1: Parámetros materiales utilizados. Los valores para la matriz ferroeléctrica reproducen aproximadamente el comportamiento sensible a la tasa de PZT policristalino a bajas frecuencias; los valores para las partículas metálicas corresponden a platino; los valores para la inclusión ferromagnética reproducen aproximadamente el comportamiento sensible a la tasa del $\mathrm{CoFe}_{2} \mathrm{O}_{4}$ a bajas frecuencias (Avellaneda y Harshe, 1994; Miehe et al., 2011b). La constante $\mu_{0}$ denota la permeabilidad del vacío.

\begin{tabular}{|c|c|c|c|}
\hline Símbolo & Parámetro & Unidades & Valor \\
\hline \multicolumn{4}{|c|}{ Matriz ferroeléctrica } \\
\hline$\epsilon$ & permitividad eléctrica & $\mathrm{C} /(\mathrm{V} \cdot \mathrm{m})$ & $1800 \epsilon_{0}$ \\
\hline$\beta$ & permitividad magnética & $\mathrm{N} / \mathrm{A}^{2}$ & $3.98 \mu_{0}$ \\
\hline$p_{s}$ & polarización de saturación & $\mathrm{C} / \mathrm{m}^{2}$ & 0.25 \\
\hline$h_{0}$ & pendiente de histéresis & $\mathrm{MV} \cdot \mathrm{m} / \mathrm{C}$ & 0.1 \\
\hline$m$ & exponente sensible a la tasa & - & 0.2 \\
\hline$\dot{p}_{0}$ & tasa de polarización de referencia & $\mathrm{C} /\left(\mathrm{m}^{2} \cdot \mathrm{s}\right)$ & 100 \\
\hline$e_{c}$ & campo eléctrico coercitivo & $\mathrm{MV} / \mathrm{m}$ & 0.35 \\
\hline$e_{0}$ & campo eléctrico de referencia & $\mathrm{MV} / \mathrm{m}$ & 0.35 \\
\hline$\gamma$ & exponente de deformación irreversible & - & 2 \\
\hline$\mu$ & módulo de Lamé & GPa & 45 \\
\hline$\lambda$ & módulo de Lamé & GPa & 70 \\
\hline$\varepsilon_{s}$ & deformación de saturación & - & 0.001 \\
\hline$\alpha_{0}$ & expansión axial piezoeléctrica & $\mathrm{MN} / \mathrm{C}$ & 12,6 \\
\hline$\alpha_{\perp}$ & expansión lateral piezoeléctrica & $\mathrm{MN} / \mathrm{C}$ & 276 \\
\hline$\alpha=$ & distorsión piezoeléctrica & $\mathrm{MN} / \mathrm{C}$ & -1460 \\
\hline \multicolumn{4}{|c|}{ Inclusión metálica } \\
\hline$\epsilon$ & permitividad eléctrica & $\mathrm{C} /(\mathrm{V} \cdot \mathrm{m})$ & $\infty$ \\
\hline$\mu$ & módulo de Lamé & $\mathrm{GPa}$ & 60 \\
\hline$\lambda$ & módulo de Lamé & GPa & 225 \\
\hline \multicolumn{4}{|c|}{ Poros } \\
\hline$\epsilon$ & permitividad eléctrica & $\mathrm{C} /(\mathrm{V} \cdot \mathrm{m})$ & $\epsilon_{0}$ \\
\hline$\mu$ & módulo de Lamé & $\mathrm{GPa}$ & 0 \\
\hline$\lambda$ & módulo de Lamé & GPa & 0 \\
\hline \multicolumn{4}{|c|}{ Inclusión ferromagnética } \\
\hline$\beta$ & polarizabilidad magnética & $\mathrm{N} / \mathrm{A}^{2}$ & $354.7 \mu_{0}$ \\
\hline$\epsilon$ & permitividad eléctrica & $\mathrm{C} /(\mathrm{V} \cdot \mathrm{m})$ & $9.52 \epsilon_{0}$ \\
\hline$m_{s}$ & magnetización saturación & $\mathrm{N} /(\mathrm{A} \cdot \mathrm{m})$ & 1.48 \\
\hline$h_{s}$ & pendiente de histéresis & $\mathrm{A}^{2} / \mathrm{N}$ & 5000 \\
\hline$m$ & exponente sensible a la tasa & - & 0.5 \\
\hline$\dot{m}_{0}$ & tasa de magnetización de referencia & $\mathrm{MN} \cdot \mathrm{s} /(\mathrm{A} \cdot \mathrm{m})$ & 10 \\
\hline$h_{c}$ & campo magnético coercitivo & $\mathrm{A} / \mathrm{m}$ & 2000 \\
\hline$h_{v 0}$ & campo magnético de referencia & $\mathrm{A} / \mathrm{m}$ & 2000 \\
\hline$\mu$ & módulo de Lamé & GPa & 51 \\
\hline$\lambda$ & módulo de Lamé & $\mathrm{GPa}$ & 173 \\
\hline$\varepsilon_{s}$ & deformación de saturación & - & 0.00025 \\
\hline$\alpha_{0}$ & expansión piezomagnética axial & $\mathrm{MA} / \mathrm{m}$ & $-2,74$ \\
\hline$\alpha_{\perp}$ & expansión piezomagnética lateral & $\mathrm{MA} / \mathrm{m}$ & $-1,3$ \\
\hline$\alpha=$ & distorsión piezomagnética & $\mathrm{MA} / \mathrm{m}$ & 2,47 \\
\hline
\end{tabular}




\subsection{Especímenes libres}

Se considera un espécimen sin restricciones mecánicas sometido a una señal eléctrica triangular con una amplitud pico de $4 e_{c}$ y una frecuencia $f_{0}$ de $1 \mathrm{~Hz}$. Se realiza un ciclado de los ejemplares partiendo de su condición sin polarizar hasta que la respuesta macroscópica alcanza un ciclo estable. Debido a que el modelo material utilizado considera la existencia de viscosidad eléctrica, una vez que finaliza el ciclado se deja al espécimen sin cargas durante cierto tiempo hasta que alcanza su estado con polarización permanente completamente relajado.

Además de los ciclos de histéresis, resulta de interés obtener las propiedades piezoeléctricas del material una vez polarizado. Para reportar estas propiedades se utiliza la notación estándar para sistemas con isotropía transversal (véase, por ejemplo, IEEE Standards, 1988)

$$
\begin{array}{ll}
d_{33}=\frac{\partial \varepsilon_{\|}}{\partial E}, & d_{31}=d_{32}=\frac{\partial \varepsilon_{\perp}}{\partial E},
\end{array}
$$

Los símbolos $\varepsilon_{\|} \mathrm{y} \varepsilon_{\perp}$ en estas expresiones se refieren a las deformaciones en las direcciones paralela y perpendicular a la dirección del campo aplicado.

\subsubsection{Ferrocerámicos con inclusiones metálicas}

En primer lugar se analizan materiales compuestos por una matriz ferroeléctrica con inclusiones de partículas metálicas. Este tipo de compuestos no solamente busca mejorar las características eléctricas del material, sino que además tiene como objetivo mejorar el desempeño mecánico del ferrocerámico puro (véase, por ejemplo, Takagi et al., 2003). En la figura 5.1 se presenta la respuesta estabilizada obtenida para ferrocerámicos con distintas fracciones volumétricas de partículas metálicas $\left(c^{(2)}=\right.$ $0,05 ; 0,15 ; 0,25)$. Se proporciona también la respuesta del ferrocerámico monolítico como referencia. En la parte a) se muestran gráficos del desplazamiento eléctrico macroscópico en la dirección del campo aplicado en función de la intensidad de campo eléctrico aplicado, normalizado mediante la polarización de saturación y el campo eléctrico coercitivo de la matriz, respectivamente. Se observa que el campo eléctrico coercitivo macroscópico del material compuesto disminuye a medida que aumenta la fracción volumétrica de partículas, como es de esperar por el mejoramiento en las características eléctricas dentro de la matriz producida por la presencia de la fase metálica. Esto se debe a que en este tipo de sistemas materiales, $\overline{\mathbf{E}}^{(2)}=\mathbf{0}$ y, por lo tanto, al considerar el balance macroscópico se tiene que $\overline{\mathbf{E}}^{(1)}=\overline{\mathbf{E}} / c^{(1)}$. 
a)

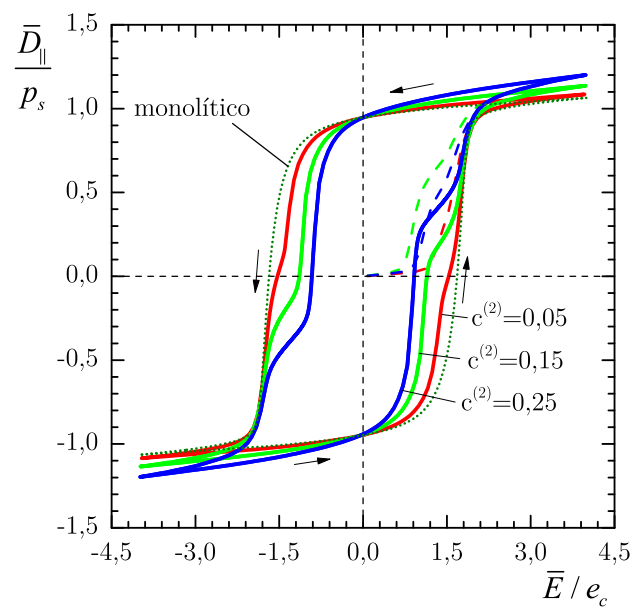

b)

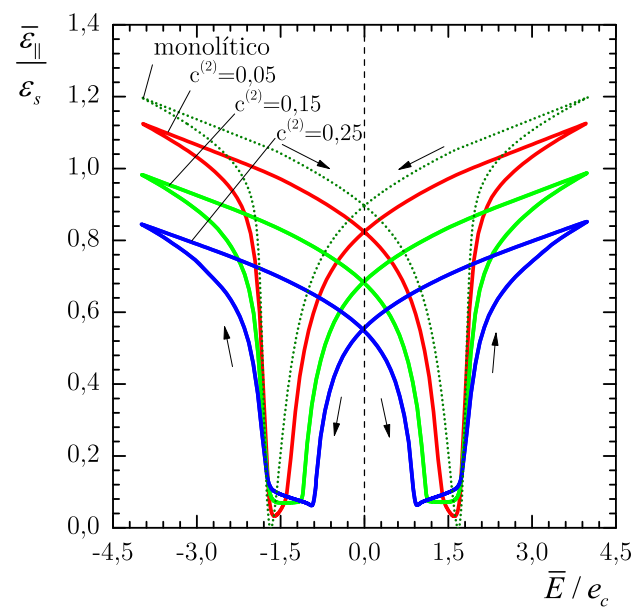

Figura 5.1: Respuesta macroscópica de ferrocerámicos con partículas metálicas a diversas fracciones volumétricas $\left(c^{(2)}=0,05 ; 0,15 ; 0,25\right)$ : a) desplazamiento eléctrico $(\bar{D})$ y b) deformación axil $(\bar{\varepsilon})$ en función de la intensidad de campo eléctrico aplicado $(\bar{E})$. La magnitudes están normalizadas con la polarización de saturación $\left(p_{s}\right)$, la deformación de saturación $\left(\varepsilon_{s}\right)$, y el campo eléctrico coercitivo $\left(e_{c}\right)$ de la matriz ferrocerámica.

La polarización macroscópica remanente, por otro lado, resulta ser insensible al agregado de partículas metálicas. Esto es consistente con las observaciones experimentales de Duan et al. (2000) en materiales compuestos de PZT con partículas de platino. Además, estas predicciones son cualitativamente idénticas a las obtenidas por Idiart (2014) para dieléctricos rígidos. En ese trabajo se había anticipado que el acoplamiento mecánico local no debería influir en la respuesta eléctrica macroscópica de especímenes libres, lo que se confirma con estos resultados.

A su vez, en la parte b) se muestran curvas para la deformación axial en la dirección del campo eléctrico aplicado, normalizado por la deformación de saturación, en función de la intensidad del campo eléctrico aplicado. La amplitud del ciclo de deformación representa la electrodeformabilidad del material compuesto. Se observa entonces que la electrodeformabilidad disminuye a medida que aumenta la fracción volumétrica de partículas. Esta deformación inducida eléctricamente está compuesta de una parte piezoeléctrica y una parte ferroeléctrica asociada a la conmutación de dominios, como se observa en la expresión simplificada (2.42). Para los procesos de carga que se han considerado aquí, la parte ferroeléctrica es dominante. La disminución de la electrodeformabilidad global es una consecuencia del hecho que la deformación ferroeléctrica en la fase de la matriz, dada por (2.35), es proporcional a la polarización a la potencia $\gamma$ y tiene como valor límite a la deformación de saturación. Entonces, a pesar de que el agregado de partículas metálicas aumenta el campo eléctrico en la fase de la matriz, no produce una electrodeformación mayor en esa fase, pero al mismo tiempo reduce la cantidad de material que exhibe acoplamiento electromecánico. Debe 
a)

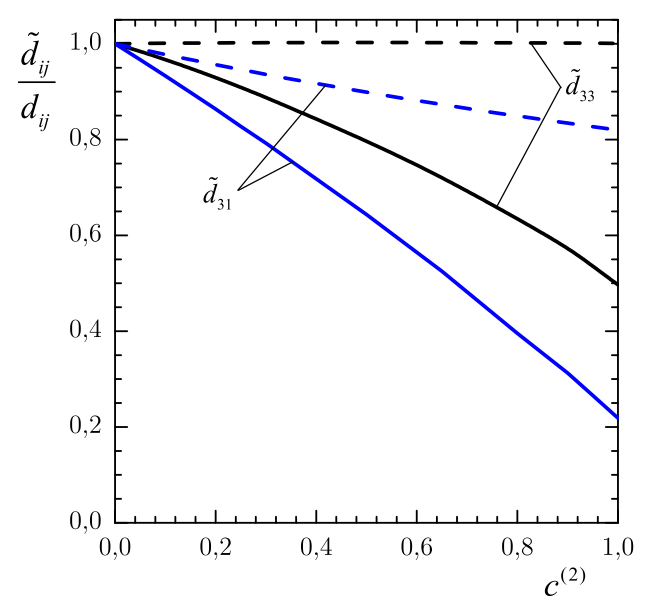

c)

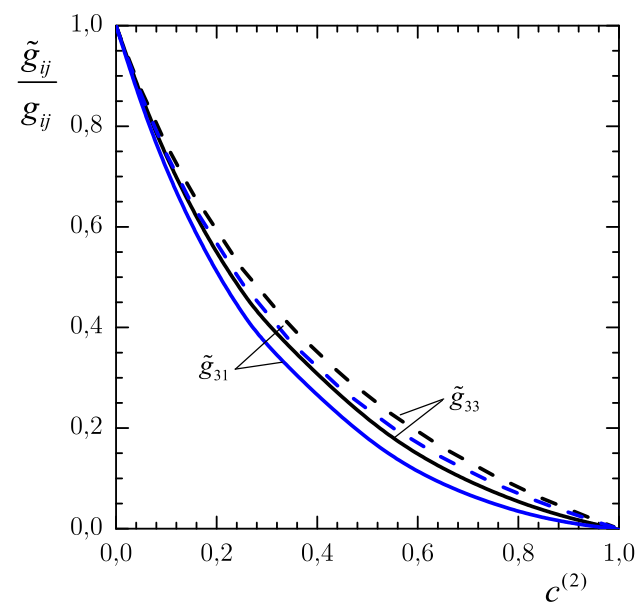

b)

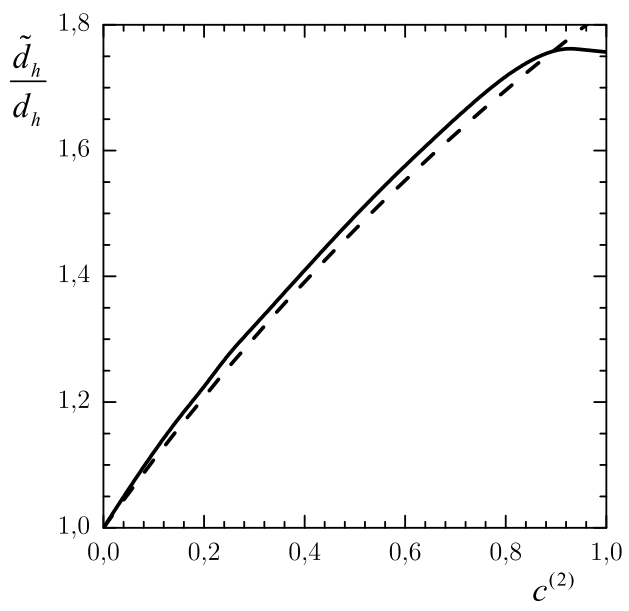

d)

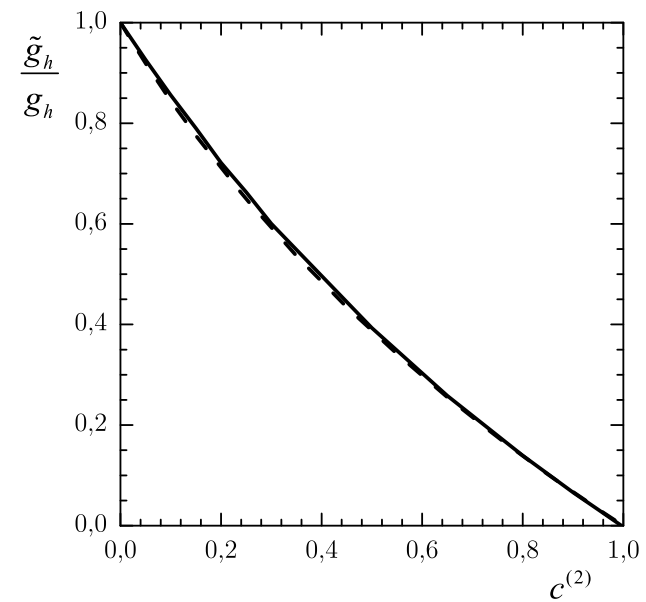

Figura 5.2: Coeficientes piezoeléctricos macroscópicos de un ferrocerámico con polarización permanente en función de la fracción volumétrica de partículas metálicas. Las líneas punteadas corresponden a la aproximación de polarización uniforme.

notarse que este mecanismo entrará en juego independientemente de las propiedades elásticas de la fase de la inclusión.

En la figura 5.2 se muestran las predicciones correspondientes a los coeficientes piezoeléctricos de ejemplares polarizados permanentemente al final del ciclo de carga, en función de la fracción volumétrica de partículas. Estos coeficientes fueron obtenidos derivando numéricamente la deformación macroscópica con respecto al campo eléctrico y al desplazamiento eléctrico. A modo de comparación, se presentan en líneas de trazos los resultados obtenidos utilizando la popular aproximación de polarización uniforme presentada en (2.45)-(2.47), utilizando los mismos parámetros materiales y la misma microgeometría de laminados secuenciales. Se observa que la adición de partículas metálicas es perjudicial para todos los coeficientes piezoeléctricos, con excepción del coeficiente hidrostático $\widetilde{d}_{h}$. Las tendencias predichas son consistentes 
con los resultados experimentales de Li et al. (2001) en cerámicos de PZT con partículas de platino. Debe considerarse que en ese estudio las muestras percolan a concentraciones del orden del $30 \%$ dado que se utiliza un único tamaño de partículas de platino. La principal observación en el contexto de esta figura es, sin embargo, que la aproximación de polarización uniforme no captura adecuadamente la influencia de las partículas metálicas en todo el conjunto de los coeficientes piezoeléctricos. Efectivamente, aunque la influencia en los coeficientes $\widetilde{g}$ es capturada adecuadamente, la influencia en los coeficientes $\widetilde{d}_{33}$ y $\widetilde{d}_{31}$ se subestima considerablemente. De hecho, la aproximación considerando polarización uniforme no logra capturar la influencia en el coeficiente $\widetilde{d}_{33}$ que, casualmente, es uno de los coeficientes más relevantes para ciertas aplicaciones. Debido a que en la aproximación de polarización uniforme es posible variar la magnitud del tensor de acoplamiento de la matriz ferrocerámica, se utilizaron distintos valores de polarización remanente para verificar su influencia en los resultados. De esta manera se ha verificado que, incluso reescalando el tensor de acoplamiento para la fase de la matriz, se obtienen los mismos resultados normalizados. Las imprecisiones anteriores pueden asociarse al hecho de haber despreciado las fluctuaciones de la polarización remanente en la fase de la matriz. Como resultado de estas fluctuaciones, se generan tensiones residuales en el material compuesto luego de someterlo al proceso de polarización. En la figura 5.3a se presentan las predicciones para los segundos momentos de la distribución de tensiones residuales en el interior de cada fase. Las distintas curvas corresponden a los segundos momentos de la norma $\|\boldsymbol{\sigma}\|$, la tensión media hidrostática $\sigma_{m}$, y la tensión equivalente de von Mises $\sigma_{e}$, que pueden calcularse a partir de las trazas de los tensores de segundo momento (4.28)-(4.29) de la siguiente manera

$$
\begin{aligned}
\left\langle\|\boldsymbol{\sigma}\|^{2}\right\rangle^{(r)} & =\mathbb{I} \cdot\langle\boldsymbol{\sigma} \otimes \boldsymbol{\sigma}\rangle^{(r)} \\
\left\langle\sigma_{m}^{2}\right\rangle^{(r)} & =\frac{1}{3} \mathbb{J} \cdot\langle\boldsymbol{\sigma} \otimes \boldsymbol{\sigma}\rangle^{(r)} \\
\left\langle\sigma_{e}^{2}\right\rangle^{(r)} & =\frac{3}{2} \mathbb{K} \cdot\langle\boldsymbol{\sigma} \otimes \boldsymbol{\sigma}\rangle^{(r)}
\end{aligned}
$$

donde $\mathbb{J}$ y $\mathbb{K}$ son los tensores estándar de proyección de cuarto orden hidrostático y de corte, de modo tal que $\mathbb{I}=\mathbb{J}+\mathbb{K}$. En vistas de estas definiciones, se verifica la igualdad $\left\langle\|\boldsymbol{\sigma}\|^{2}\right\rangle^{(r)}=3\left\langle\sigma_{m}^{2}\right\rangle^{(r)}+(2 / 3)\left\langle\sigma_{e}^{2}\right\rangle^{(r)}$. Los gráficos corresponden a la raíz cuadrada de esas magnitudes, normalizadas por $\mu \varepsilon_{s}$. Las líneas de trazos corresponden a la inclusión y las líneas continuas a la matriz. Puede observarse que para fracciones volumétricas de partículas bajas a moderadas las tensiones residuales son, en promedio, mayores en la inclusión que en la matriz, mientras que lo opuesto se verifica para fracciones volumétricas grandes. Teniendo en cuenta la magnitud de $\mu \varepsilon_{s}$ para los parámetros materiales de la fase ferroeléctrica de la tabla 5.1, estos niveles de tensión 
a)

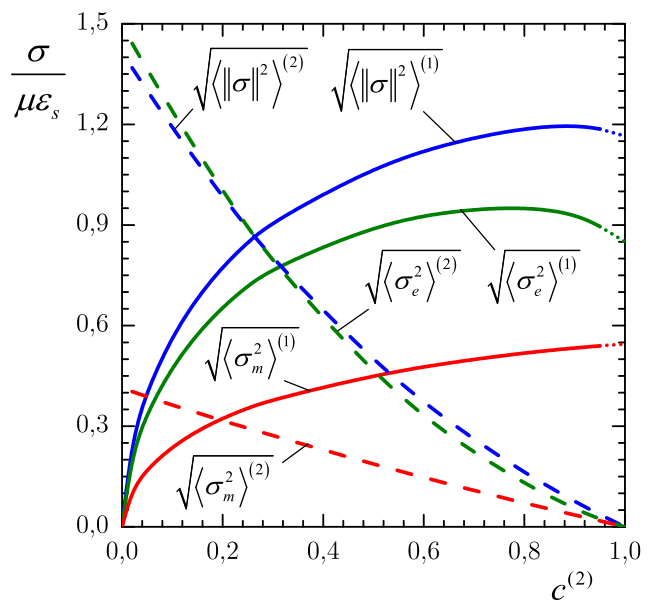

b)

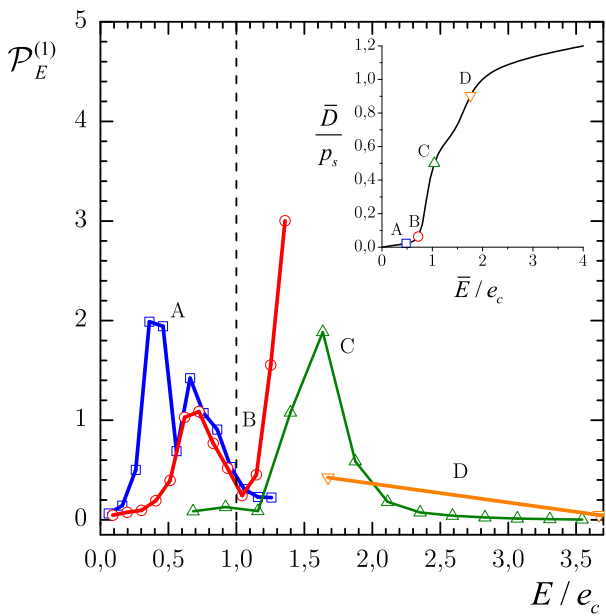

Figura 5.3: Estadísticas de campo en ferrocerámicos con partículas metálicas: a) segundos momentos de la distribución de tensiones residuales en las fases en función de la fracción volumétrica de partículas, b) función de densidad de probabilidad para la distribución de intensidad de campo eléctrico dentro de la matriz para diversos valores de campo eléctrico aplicado, para $c^{(2)}=0,25$. Las líneas de trazos en a) corresponden a la inclusión y las líneas continuas a la matriz.

están muy por debajo de la resistencia mecánica de las partículas metálicas y de la interfaz matriz-partícula, pero pueden ser del orden de la resistencia mecánica de la matriz ferrocerámica en tracción. En relación con esto, se observa que las tensiones hidrostáticas presentes en la matriz son mayormente de signo positivo. En cualquier caso, la aproximación de polarización uniforme desprecia estas tensiones residuales completamente y por lo tanto no permite utilizar ningún criterio de rotura. Cabe mencionar además que, a pesar de que los niveles de tensión en la fase ferroeléctrica son de magnitud considerable, no se evidencian en la respuesta macroscópica del material compuesto problemas como los encontrados en el capítulo 3. En este caso el material a nivel local se encuentra bajo un estado de carga multiaxial, muy distinto de las condiciones de carga uniaxiales para las cuales se obtuvieron los valores de referencia para el comienzo del problema en el material monolítico. Si bien no se descarta la posibilidad que en algunas zonas del material se den las condiciones necesarias para el surgimiento del problema, este fenómeno no llega a manifestarse a nivel macroscópico, obteniéndose una respuesta físicamente admisible.

Se concluye esta discusión haciendo notar que, a diferencia del ferrocerámico monolítico, la respuesta eléctrica del material compuesto mostrada en la figura 5.1a presenta una saturación en dos etapas que se vuelve más pronunciada a medida que aumenta la fracción volumétrica de partículas. Esta característica fue observada - pero no explicada - por Idiart (2014) en el contexto de compuestos rígidos y este tipo de respuesta también ha sido encontrada en simulaciones de fase completa en materiales 
compuestos periódicos (Keip et al., 2015). Este atributo puede atribuirse al carácter bimodal de la intensidad de campo eléctrico dentro de la matriz. En la figura 5.3b se muestra la función de densidad de probabilidad $\mathcal{P}_{E}^{(1)}(E)$ de la intensidad de campo eléctrico $E(\mathbf{x})=|\mathbf{E}(\mathbf{x})|$ en la matriz, para cuatro valores de campo eléctrico aplicado en la rampa de carga inicial desde el estado sin polarización — véase el recuadro en la figura $5.3 \mathrm{~b}$ - para la concentración elegida de $c^{(2)}=0,25$. La función $\mathcal{P}_{E}^{(1)}(E)$ es tal que $\mathcal{P}_{E}^{(1)}(E) \mathrm{d} E$ es la fracción volumétrica de fase $r=1$ donde la variable $E$ toma valores en el rango $E$ y $E+\mathrm{d} E$. Las densidades de probabilidad fueron calculadas a partir del conjunto de campos eléctricos que minimizan (4.17) haciendo uso de las expresiones de Idiart et al. (2006). Para poder tener un conjunto lo suficientemente grande de valores de campo locales, estas funciones se obtuvieron con laminados secuenciales de rango 3000. El carácter bimodal de la distribución y su evolución con la carga aplicada se observa claramente. Así, para el estado A, los dos picos se dan para intensidades de campo eléctrico por debajo del campo eléctrico coercitivo, de modo que la matriz se encuentra mayormente sin polarización permanente y la respuesta es lineal. Para el estado B, el primer pico se encuentra por encima del nivel del campo coercitivo de modo que una gran parte de la matriz está experimentando conmutación de dominios y la respuesta macroscópica incrementa su pendiente repentinamente. En el estado C, el primer pico se encuentra a una intensidad de campo eléctrico para la cual la matriz comienza a saturar $\left(\sim 1,5 e_{c}\right)$, por lo que la respuesta macroscópica exhibe una primera etapa de saturación, mientras que el segundo pico aún está por debajo del campo coercitivo. A medida que el segundo pico crece más allá del campo coercitivo, más matriz experimenta conmutación de dominios y, consecuentemente, la respuesta macroscópica aumenta su pendiente una vez más. Finalmente, en el estado $\mathrm{D}$, toda la distribución de campo eléctrico se encuentra bien por encima del campo coercitivo, de modo que la respuesta macroscópica exhibe una segunda y última saturación. La distribución bimodal también es responsable de la distorsión de los ciclos de histéresis a medida que aumenta la fracción volumétrica de partículas en la figura 5.1b. Este tipo de densidades de campo eléctrico bimodales también pueden encontrarse en dieléctricos rígidos ideales con microgeometrías de Hashin, donde los picos pueden asociarse a singularidades del tipo van Hove de la distribución del campo eléctrico en la matriz (Cule y Torquato, 1998). Estas microgeometrías corresponden a distribuciones de partículas polidispersas como la de la microgeometría de laminados secuenciales considerada aquí. Por el contrario, la función de densidad de campo eléctrico en dieléctricos rígidos ideales con distribución de partículas monodispersa resulta ser unimodal (Cheng y Torquato, 1997), por lo que no se espera que ocurra una saturación en dos etapas en la respuesta macroscópica. Esta es posiblemente la razón por la cual los resultados experimentales de Duan et al. (2000) para compuestos de PZT no exhiben esta característica. En cualquier caso, conocer el carácter de la 
a)

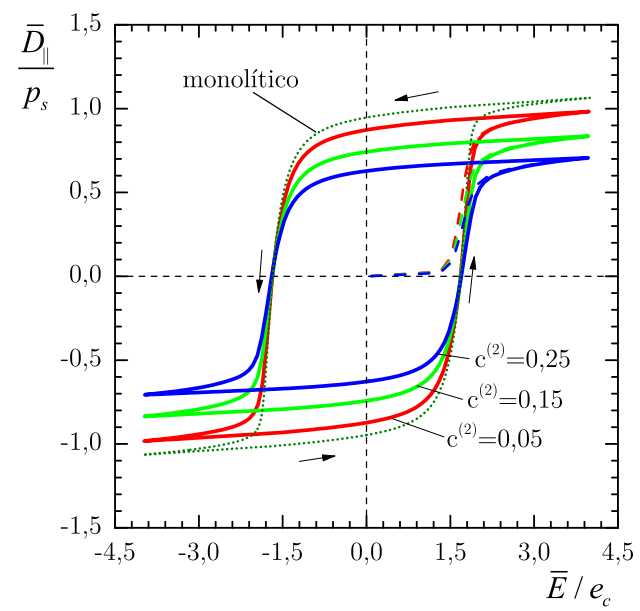

b)

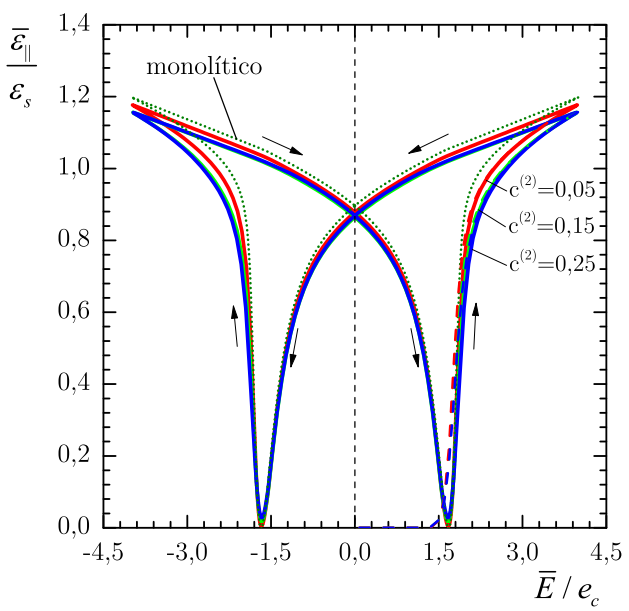

Figura 5.4: Respuesta macroscópica de ferrocerámicos conteniendo microcavidades a diversas fracciones volumétricas $\left(c^{(2)}=0,05 ; 0,15 ; 0,25\right)$ : a) desplazamiento eléctrico $(\bar{D})$ y b) deformación axial $(\bar{\varepsilon})$ en función de la intensidad de campo eléctrico aplicado $(\bar{E})$. La magnitudes están normalizadas con la polarización de saturación $\left(p_{s}\right)$, la deformación de saturación $\left(\varepsilon_{s}\right)$, y el campo eléctrico coercitivo $\left(e_{c}\right)$ de la matriz ferrocerámica.

densidad de probabilidad de los campos puede resultar útil no solo para comprender la respuesta observada, sino también para derivar aproximaciones de campo promedio siguiendo las ideas de Pellegrini (2001).

\subsubsection{Ferrocerámico con microcavidades}

Se consideran aquí materiales ferroeléctricos con microcavidades o poros. Este tipo de configuración material presenta excelentes propiedades electromecánicas, en particular el factor de mérito hidrostático (HFOM), lo que las hace especialmente adecuadas para aplicaciones en hidrófonos de baja frecuencia (véase Marselli et al., 1999; Nie et al., 2010, y sus referencias).

En la figura 5.4 se presentan las predicciones para materiales con diversos niveles de porosidad $\left(c^{(2)}=0,05 ; 0,15 ; 0,25\right)$. Nuevamente se ha agregado la respuesta del material ferrocerámico monolítico como referencia. En la parte a) se muestran curvas normalizadas para el desplazamiento eléctrico macroscópico en función de la intensidad de campo eléctrico aplicado. Se observa que el campo coercitivo macroscópico es relativamente insensible a la porosidad, mientras que la polarización remanente disminuye a medida que aumenta la porosidad. Esto es exactamente lo opuesto a lo observado en la sección anterior para inclusiones metálicas, y es consistente con las observaciones experimentales de Zeng et al. (2007) y Nie et al. (2010) en cerámicos de PZT porosos. Además, las predicciones son cualitativamente idénticas 
a)

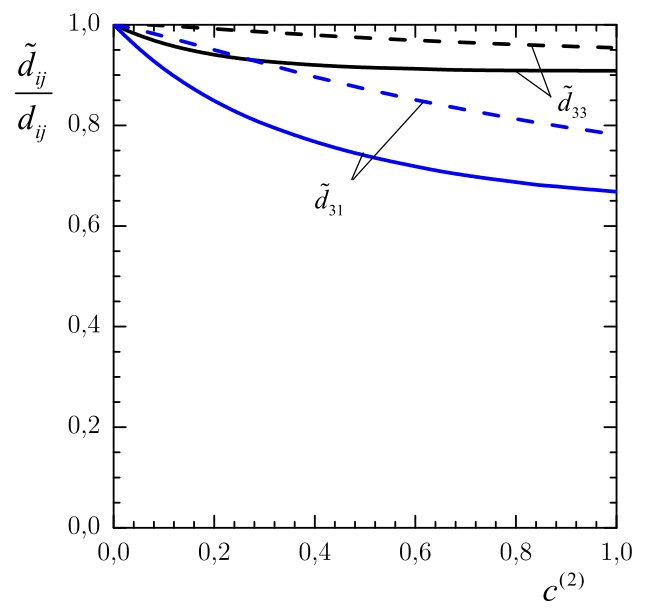

c)

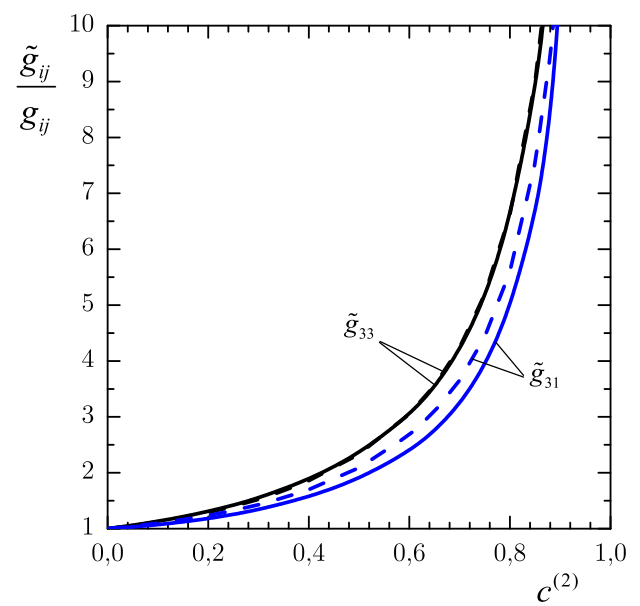

b)

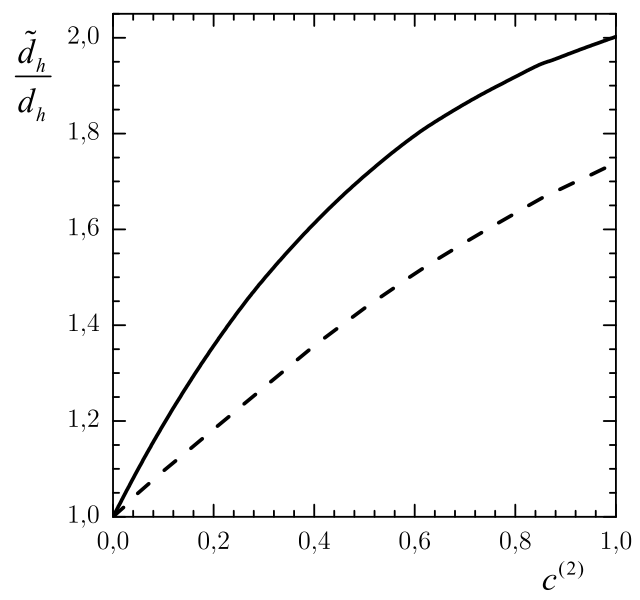

d)

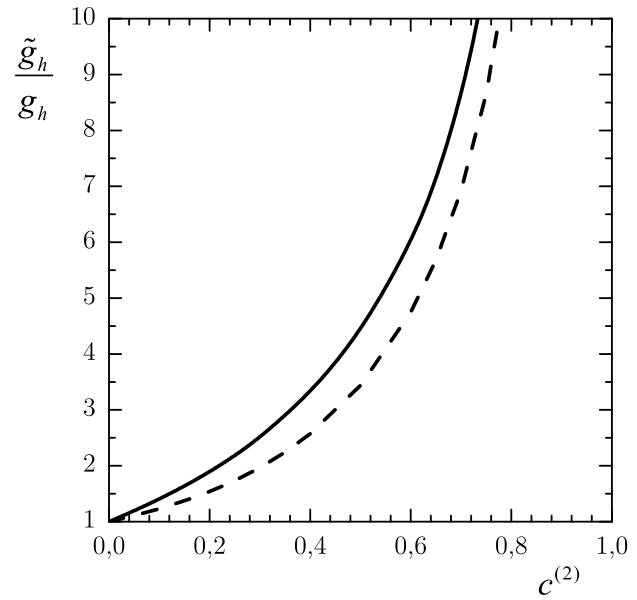

Figura 5.5: Coeficientes piezoeléctricos macroscópicos de un ferrocerámico con polarización permanente en función de la porosidad. Las líneas punteadas corresponden a la aproximación de polarización uniforme.

a las presentadas por Idiart (2014) para dieléctricos rígidos, lo que confirma que el acoplamiento electromecánico local no influye en la respuesta eléctrica macroscópica de especímenes libres para deformar.

A su vez, en la parte b) se muestran curvas para la deformación axial en la dirección del campo eléctrico aplicado en función de la intensidad de campo eléctrico aplicada. A diferencia del caso con partículas metálicas, la electrodeformabilidad del ejemplar no parece verse afectada con la porosidad. Sin embargo, los coeficientes piezoeléctricos de los ejemplares polarizados permanentemente si varían fuertemente con la porosidad, como puede verse en la figura 5.5. La presencia de porosidad resulta ser beneficiosa para todos los coeficientes $\widetilde{g}$ y para el coeficiente hidrostático $\widetilde{d}_{h}$. Las tendencias son consistentes con los resultados experimentales disponibles para ferrocerámicos con porosidad cerrada (Topolov y Bowen, 2009). Debe enfatizarse, sin embargo, que 
los resultados no son aplicables a sistemas materiales con porosidad abierta, donde se espera que exista una influencia mucho mayor en las propiedades macroscópicas (Barolin et al., 2014; Kara et al., 2003). Por último, se observa que, al igual que en el caso de las partículas metálicas, la aproximación de polarización uniforme logra capturar las tendencias adecuadamente, siendo bastante precisas para los coeficientes

$\tilde{g}$, pero subestima la influencia de la porosidad en los coeficientes $\widetilde{d}_{33}$ y $\widetilde{d}_{31}$. Esto último parece ser una imprecisión común introducida por la aproximación, al menos en el contexto de medios particulados.

En la figura 5.6a se presentan gráficas de los segundos momentos de la distribución de tensiones residuales dentro la matriz únicamente, dado que las microcavidades no experimentan tensiones. En general, se observan las mismas tendencias que para el caso de partículas metálicas, con las tensiones hidrostáticas siendo principalmente de signo positivo. Sin embargo, al comparar con la figura 5.3a se observa que las tensiones residuales en la matriz ferroeléctrica son, en promedio, más bajas en presencia de microcavidades que en presencia de partículas metálicas. Esto es opuesto a lo que ocurre en materiales compuestos sometidos a esfuerzos mecánicos, donde fases de inclusión más rígidas inducen niveles de tensión más bajos en la matriz — véase, por ejemplo, Idiart et al. (2006). Nuevamente y a pesar de la existencia de tensiones en la matriz ferroeléctrica, no se evidencian inestabilidades en la respuesta macroscópica del material compuesto, a diferencia de lo observado en el capítulo 3 para el cerámico ferroeléctrico.

Se puede concluir la discusión haciendo notar que la repuesta eléctrica macroscópica de la figura 5.4a no presenta la saturación en dos etapas ni la distorsión en los ciclos de histéresis que se observan para el caso de partículas metálicas. En la figura 5.3b se presenta la función de densidad de probabilidad de la intensidad de campo eléctrico dentro de la matriz para cuatro valores del campo eléctrico aplicado, donde se observa claramente su carácter unimodal. La ausencia de una saturación en dos etapas es entonces consistente con el mecanismo identificado en la sección anterior.

\subsubsection{Ferrocerámicos con inclusiones ferromagnéticas}

Se considera ahora un sistema material multiferróico, formado por una matriz ferroeléctrica con inclusiones ferromagnéticas. Si bien en el capítulo 2 se presentaron algunas funciones termodinámicas específicas para cargas electro o magnetomecánicas y teniendo en cuenta que ninguna de las dos fases es en sí misma multiferróica, se considera un efecto aditivo de las energías para contemplar la contribución completa de cada una de las fases frente a cargas eletromagnetomecánicas. Esto equivale a contemplar en las densidades de energía definidas en (2.31) y (2.49) la contribución 
a)

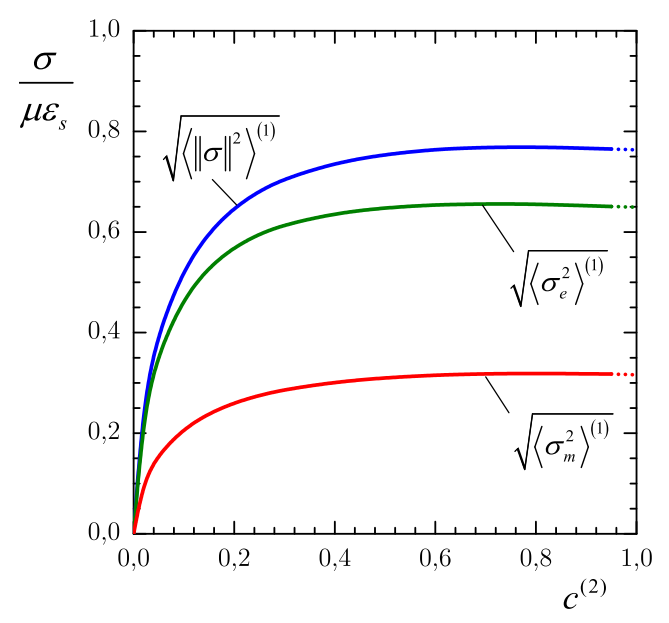

b)

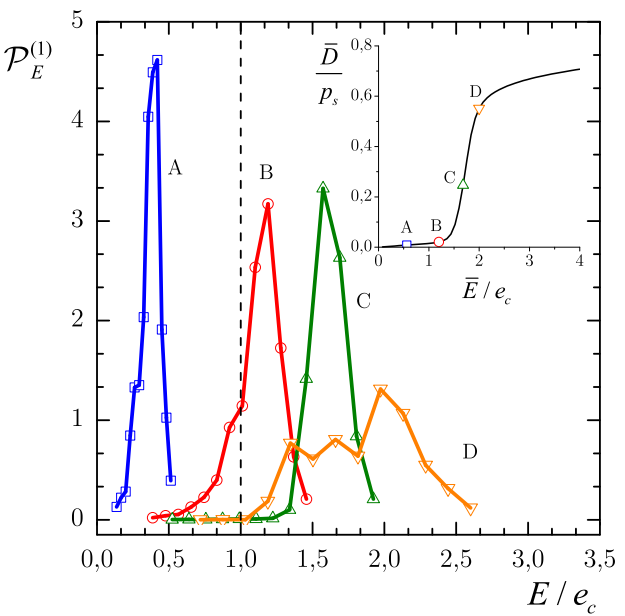

Figura 5.6: Estadísticas de campo en ferrocerámicos con microcavidades: a) segundos momentos de la distribución de tensiones residuales en la matriz en función de la porosidad, b) función de densidad de probabilidad para la distribución de intensidad de campo eléctrico dentro de la matriz para diversos valores de campo eléctrico aplicado, para $c^{(2)}=0,25$.

de la energía de magnetización y de polarización, respectivamente. De este modo, la densidad de energía para la fase ferroeléctrica toma la forma

$$
\begin{aligned}
\mathfrak{e}(\boldsymbol{\varepsilon}, \mathbf{P}, \mathrm{p}, \mathbf{M})= & \frac{1}{2}(\boldsymbol{\varepsilon}-\hat{\boldsymbol{\varepsilon}}(\mathrm{p})) \cdot \mathbb{C}(\boldsymbol{\varepsilon}-\hat{\boldsymbol{\varepsilon}}(\mathrm{p}))+\frac{1}{2}(\mathbf{P}-\mathrm{p}) \cdot \boldsymbol{\kappa}(\mathbf{P}-\mathrm{p})+ \\
& +(\boldsymbol{\varepsilon}-\hat{\boldsymbol{\varepsilon}}(\mathrm{p})) \cdot \mathbf{h}(\mathrm{p})(\mathbf{P}-\mathrm{p})+\mathfrak{e}_{p d}(\mathrm{p})+\frac{1}{2} \mathbf{M} \cdot \boldsymbol{\beta}^{-1} \mathbf{M}
\end{aligned}
$$

mientras que para la fase ferromagnética toma la forma

$$
\begin{array}{r}
\mathfrak{e}(\varepsilon, \mathbf{M}, \mathbf{m}, \mathbf{P})=\frac{1}{2}(\varepsilon-\hat{\varepsilon}(\mathbf{m})) \cdot \mathbb{C}(\varepsilon-\hat{\varepsilon}(\mathbf{m}))+\frac{1}{2}(\mathbf{M}-\mathbf{m}) \cdot \boldsymbol{\beta}^{-1}(\mathbf{M}-\mathbf{m})+ \\
+(\varepsilon-\hat{\varepsilon}(\mathbf{m})) \cdot \mathbf{h}(\mathbf{m})(\mathbf{M}-\mathbf{m})+\mathfrak{e}_{p d}(\mathbf{m})+\frac{1}{2} \mathbf{P} \cdot \boldsymbol{\kappa} \mathbf{P} .
\end{array}
$$

Como ya fue mencionado en el capítulo 1, este tipo de materiales mutifuncionales son de gran interés por su potencial de aplicación (Nan et al., 2008). Siguiendo el caso de estudio presentado por Miehe et al. (2011b) para un compuesto multiferróico, se considera un espécimen con una concentración de fase ferromagnética $c^{(2)}=0,1$ sin restricciones mecánicas y sometido a una señal eléctrica triangular con una amplitud pico de $4 e_{c}$ y una frecuencia $f_{0}$ de $1 \mathrm{~Hz}$. Solamente se somete al espécimen a medio ciclo de carga, con el objetivo de polarizar la fase ferroeléctrica. La idea es posteriormente someter al material a un campo magnético para magnetizar la fase ferromagnética y luego recién proceder al estudio de las propiedades multiferróicas del material compuesto. Sin embargo, durante la etapa de polarización se obtienen resultados que no resultan físicamente aceptables por lo que la presentación y análisis 
a)

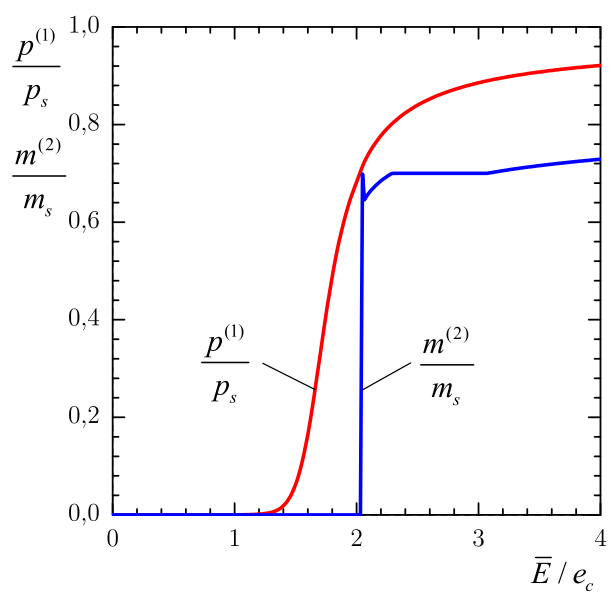

c)

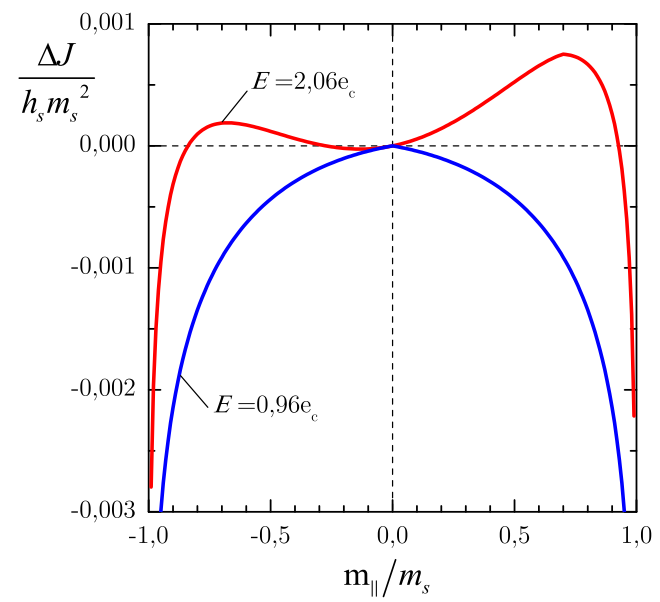

b)

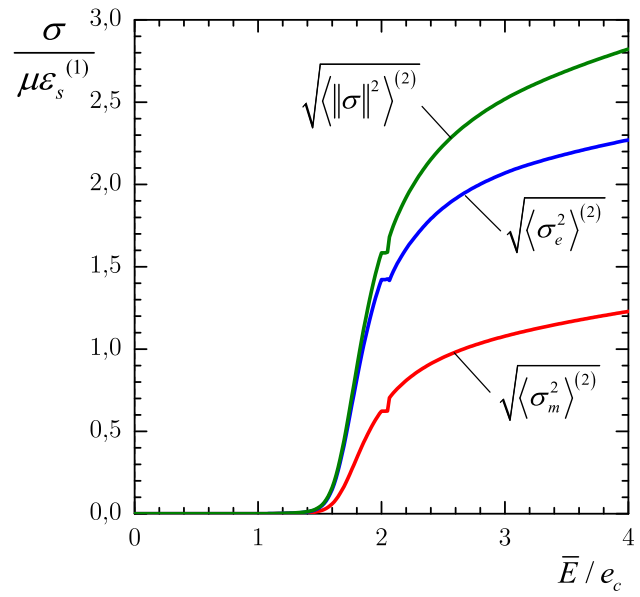

d)

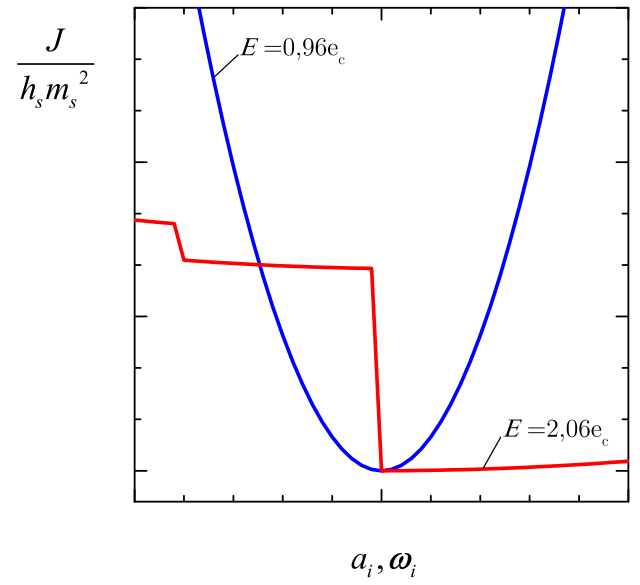

Figura 5.7: Respuesta macroscópica de un ferrocerámico con inclusiones ferromagnéticas: a) evolución de la polarización y magnetización irreversibles en cada fase; b) evolución de las tensiones en la inclusión ferromagnética; c) función objetivo para el cálculo de la magnetización remanente y d) función objetivo de la minimización externa (4.17).

de los resultados se centra en esta etapa. Los parámetros materiales presentados en la tabla 5.1 para la inclusión magnética corresponden a la aleación $\mathrm{CuFe}_{2} \mathrm{O}_{4}$ en lugar del Terfenol-D utilizado en la sección 3.3 debido a que la combinación de parámetros de ambas fases permite evidenciar la problemática de la simulación más claramente. En la figura 5.7a se presenta la evolución de la polarización irreversible promedio $\overline{\mathrm{p}}^{(1)}$ en la matriz ferrocerámica y de la magnetización irreversible $\mathbf{m}^{(2)}$ en la inclusión ferromagnética. Debe recordarse que en la microgeometría de laminados secuenciales considerada, los campos son constantes en todas las inclusiones. Estos gráficos muestran la evolución de las proyecciones paralela del vector $\overline{\mathrm{p}}^{(1)}$ y $\mathbf{m}^{(2)}$ respecto al eje de aplicación de la carga, en función del campo eléctrico aplicado. Como es de esperarse, se observa que a medida que el campo eléctrico aplicado aumenta por encima del 
valor del campo eléctrico coercitivo de la matriz, comienza a nuclearse polarización irreversible en la dirección del campo aplicado. En la inclusión ferromagnética, por otro lado, se observa una nucleación repentina de magnetización irreversible, incluso cuando no se ha aplicado aún el campo magnético externo. Teniendo en cuenta que la fase ferromagnética no exhibe en si misma características multiferróicas, esta nucleación de magnetización irreversible no resulta físicamente admisible y se debe, nuevamente, a la falta de convexidad en la densidad de energía del modelo utilizado.

La explicación de este fenómeno puede encontrarse analizando lo que sucede en el material compuesto a medida que el campo eléctrico comienza a aumentar más allá del valor de campo coercitivo de la matriz. Asociada al surgimiento de la polarización irreversible en la matriz, se generan deformaciones en la fase ferrocerámica de acuerdo a la relación (2.35). Esta deformación en la matriz induce deformaciones y, consecuentemente, tensiones mecánicas en la fase de la inclusión ferromagnética. En la figura 5.7b se presenta la evolución de los segundos momentos de las tensiones en la fase ferromagnética. Las magnitudes de estas tensiones dependerán, lógicamente,0 del contraste entre las propiedades mecánicas de las fases y de sus concentraciones. Independientemente de esto, son estas tensiones las responsables del comportamiento espurio exhibido por la fase ferromagnética y responden al fenómeno discutido en 3.3.2, dónde tensiones mecánicas inducían la aparición de una magnetización irreversible. Efectivamente, si se analiza la función objetivo siendo maximizada para obtener la evolución de la magnetización permanente para distintos valores de campo eléctrico externo aplicado (figura 5.7c), se observa el mismo fenómeno presentado en la sección 3.3.2. En efecto, para un nivel de campo eléctrico - y consecuentemente tensión - bajos, la función siendo maximizada es convexa y posee un único máximo en $\mathbf{m}=\mathbf{0}$. A medida que el campo eléctrico externo aumenta, también lo hace el nivel de tensión en la inclusión, lo que favorece el desarrollo de máximos locales hasta que eventualmente uno de ellos pasa a ser un máximo local y se inducen la nucleación repentina de magnetización observada.

Si se analiza en detalle la figura 5.7b, se observa que para un campo aplicado de aproximadamente $2 e_{c}$ las curvas de tensiones permanecen constantes por un breve periodo de tiempo, justo antes de que se produzca el salto en la magnetización irreversible de la inclusión. Este comportamiento tampoco resulta ser físicamente correcto dado que la evolución para las tensiones debería estar directamente asociada a la evolución de la polarización irreversible, la cual se encuentra en crecimiento en ese momento. Este fenómeno nuevamente se debe a la no convexidad en la densidad de energía de la fase ferromagnética y se pone en evidencia afectando la función objetivo de la minimización (4.17) correspondiente a la obtención de los campos eléctrico, magnético y de tensiones en las fases. En efecto, en la figura 5.7d se muestra a manera 
de ejemplo la variación de esta función con respecto a alguno de los parámetros $a_{i} \mathrm{O}$ $w_{i}$ para distintos valores de campo eléctrico externo aplicado. Se observa como la pérdida de convexidad en la densidad de energía tiene asociados saltos en la función objetivo (4.17), con lo que el óptimo obtenido no es físicamente representativo.

Se concluye entonces que incluso cuando no se considere un estado de tensiones mecánicas externas aplicadas al sistema material, naturalmente las distintas fases van a estar sometidas a tensiones mecánicas y dependiendo de la combinación de parámetros materiales utilizados, pueden hacer inviable el uso de modelos como el considerado aquí para el estudio de sistemas multiferróicos.

\subsection{Especímenes bajo compresión}

En esta sección se consideran especímenes bajo la acción simultánea de campos eléctricos y tensiones mecánicas. El PZT policristalino puede soportar tensiones de compresión muy por encima de los 50MPa (Munz et al., 1998). Por esta razón y a modo de ejemplo, se someten a especímenes sin polarizar a una carga uniaxial de compresión de 50MPa, seguida por una señal eléctrica triangular a lo largo de la dirección de aplicación del esfuerzo mecánico con amplitud pico de $4 e_{c} \mathrm{y}$ una frecuencia de $1 \mathrm{~Hz}$. Las predicciones resultantes para sistemas materiales con partículas metálicas y microcavidades con una fracción volumétrica de $c^{(2)}=0,25$ se reportan en la figura 5.8 .

En las partes a) \& b) se presenta el desplazamiento eléctrico macroscópico y en las partes c) \& d) se muestra la deformación macroscópica axial en función del campo eléctrico aplicado para ambos sistemas materiales. Las líneas continuas corresponden a los ferrocerámicos bifásicos, mientras que las líneas punteadas corresponden a la respuesta del ferrocerámico monolítico y corresponden a la respuesta estable previo a la aparición de la inestabilidad discutida en la sección 3.2. Además, se muestran dos conjuntos de líneas continuas: las líneas azules corresponden a la respuesta para bajos ciclos de cargas, mientras que las líneas rojas corresponden a las respuestas estabilizadas a gran número de ciclos. La principal observación en el contexto de estas figuras es que, en contraste con los resultados para especímenes libres, la respuesta estabilizada a gran número de ciclos difiere considerablemente de la respuesta para bajo número de ciclos, especialmente para los especímenes porosos. Estas diferencias, sin embargo, no resultan físicamente significativas por la simetría del problema, sino que son un resultado espurio de la definición para la densidad de energía que caracteriza a la matriz ferrocerámica. Este problema está en correspondencia con los resultados obtenidos en el capítulo 3 para especímenes bajo cargas mecánicas, y dado que la densidad de energía (2.31) no es convexa como lo requiere el marco de 
a)

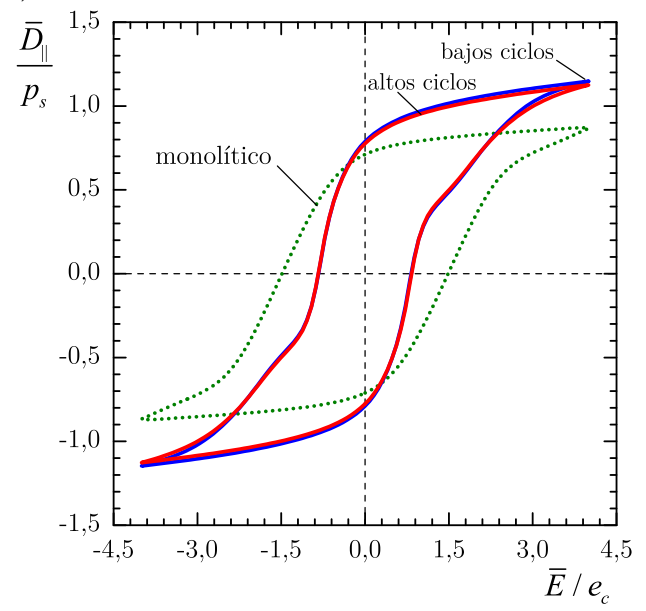

c)

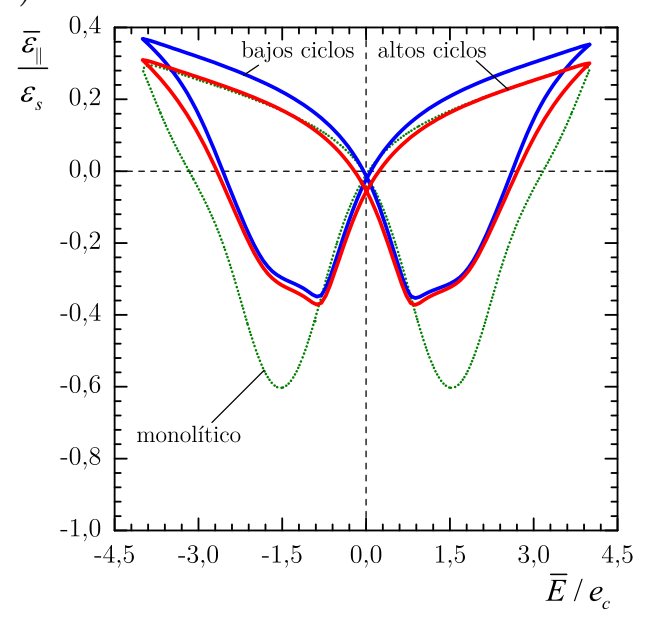

e)

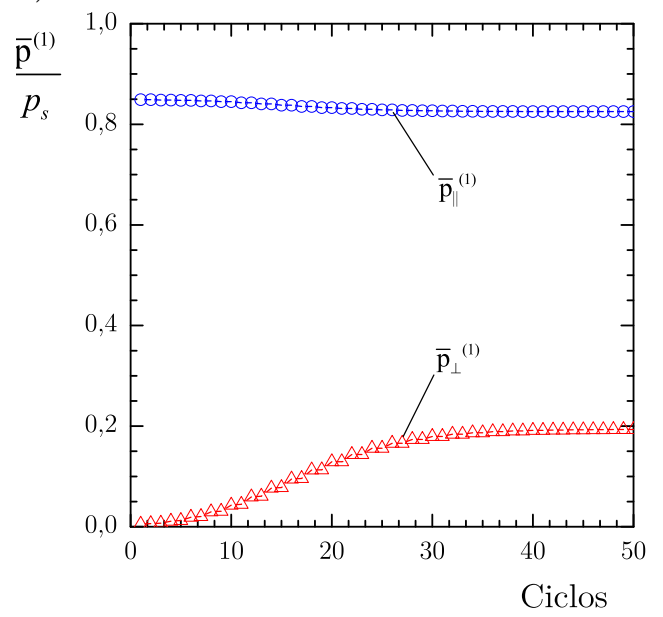

b)

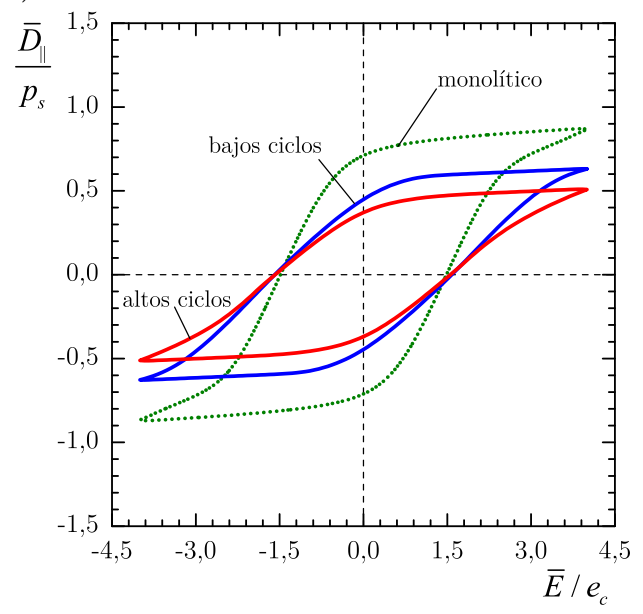

d)

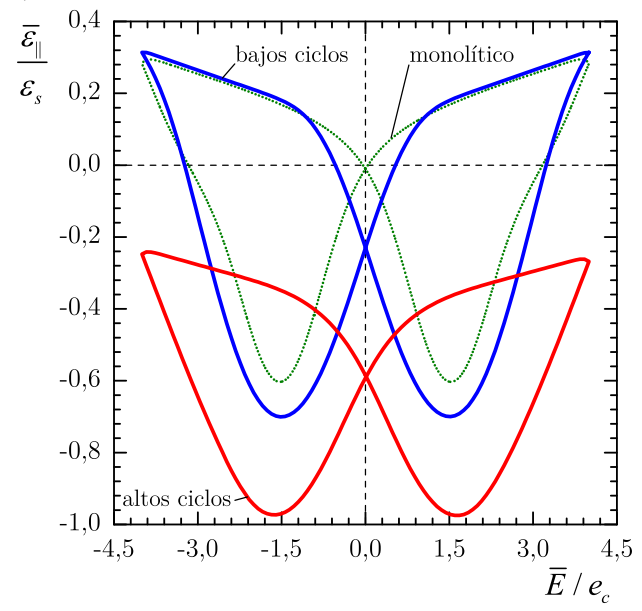

f)

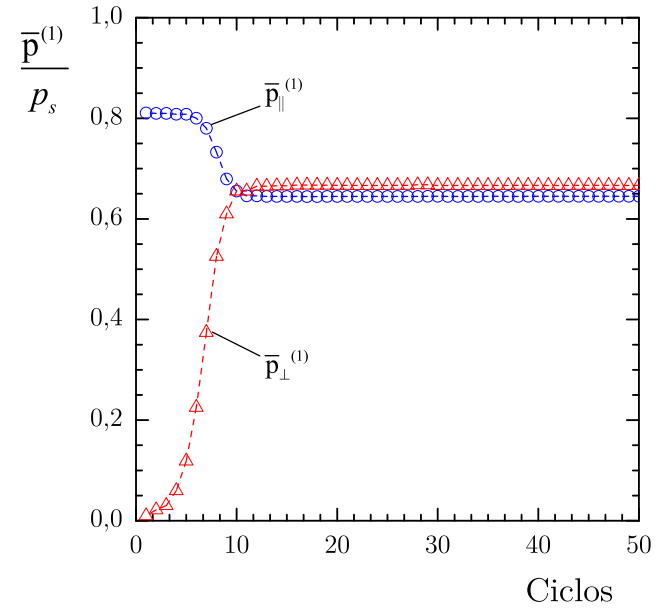

Figura 5.8: Desplazamiento eléctrico macroscópico $(\bar{D})$, deformación axial macroscópica $\left(\bar{\varepsilon}_{\|}\right)$y polarización irreversible promedio en la matriz $\left(\overline{\mathrm{p}}^{(1)}\right)$ de especímenes comprimidos con a) \& c) \& e) partículas metálicas y b) \& d) \& f) microcavidades, con una fracción volumétrica de $c^{(2)}=0,25$. 
materiales estándar generalizados, la unicidad de la solución al problema incremental no está garantizada.

Precisamente es una falta de unicidad en la evolución de la polarización irreversible lo que resulta en el extraño comportamiento presentado anteriormente. Gráficos para la polarización irreversible promedio $\overline{\mathrm{p}}^{(1)}$ en la matriz ferrocerámica se muestran en las partes e) \& f). Estos gráficos muestran el máximo valor para cada ciclo eléctrico de las proyecciones paralela y perpendicular del vector $\overline{\mathrm{p}}^{(1)}$ respecto al eje de aplicación de la carga, en función del número de ciclos. En vistas de la isotropía global de los especímenes sin polarizar, el vector $\overline{\mathrm{p}}^{(1)}$ debe permanecer alineado con el eje de las cargas aplicadas a lo largo del proceso de carga, es decir, la proyección perpendicular debe ser nula. Sin embargo, las predicciones muestran un crecimiento exponencial de la proyección perpendicular hasta alcanzar un valor de saturación finito impuesto por el requisito global que $|\mathrm{p}(\mathbf{x})|<p_{s}$. En la sección 3.2 se ha demostrado que este fenómeno es efectivamente consecuencia de la no convexidad en la densidad de energía (2.31), introducida por la relación (2.35) entre la deformación irreversible y la polarización.

Resulta entonces que, incluso para niveles de tensión por debajo del límite material de la fase ferroeléctrica, se presentan comportamientos que no resultan ser físicamente admisibles, requiriéndose un análisis exhaustivo para asegurar la validez de las predicciones para condiciones de carga arbitrarias. 


\section{Teoría multiescala para un dieléctrico con fases semiconductoras}

\subsection{Introducción}

En los capítulos anteriores se han analizado los materiales ferrocerámicos bajo la hipótesis fundamental de considerarlos como dieléctricos aislantes. Sin embargo y como ya fue mencionado, muchos de los materiales ferroeléctricos de interés son en realidad semiconductores de banda prohibida ancha y las cargas libres y vacancias en la microestructura afectan de manera no trivial su comportamiento (Xiao y Bhattacharya, 2008). Dependiendo de la configuración del sistema material y su aplicación, contar con herramientas que tengan en cuenta la presencia de iones móviles es fundamental para poder caracterizar y modelar adecuadamente su respuesta. Para el caso de materiales ferroeléctricos, este problema es muy complejo debido a que la polarización y las cargas eléctricas interactúan a través del potencial electrostático en el material de manera no trivial. Es por este motivo que se procede a realizar un análisis preliminar de la problemática centrando la atención en la presencia de iones libres en el material sin tener en cuenta la naturaleza ferroeléctrica del mismo. De este modo se espera poder desarrollar el marco teórico necesario para analizar y así obtener una mejor comprensión del fenómeno y de las herramientas necesarias para resolver el problema, sentando las bases para la incorporación de la ferroelectricidad.

\subsection{Marco teórico}

\subsubsection{Sistema material}

El sistema material bajo estudio se idealiza como un cuerpo heterogéneo que contiene una única especie de iones móviles. Específicamente se consideran especímenes compuestos por un gran número de dominios microestructurales uniformemente distribuidos, que pueden diferir en forma y composición y que están formados por un material polar eléctricamente neutro que contienen una única especie de cationes móviles - y contraiones fijos de igual valencia - que son incapaces de moverse a través de los bordes de los dominios, véase la figura 6.1. Cabe mencionar, sin embargo, que la formulación puede adaptarse a sistemas más complejos. Los especímenes se cargan electroestáticamente bajo condiciones isotérmicas a través de electrodos superficiales delgados adheridos a su superficie exterior. 
a)

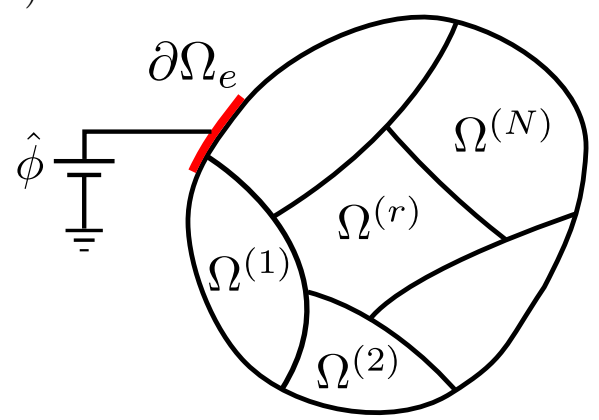

b)

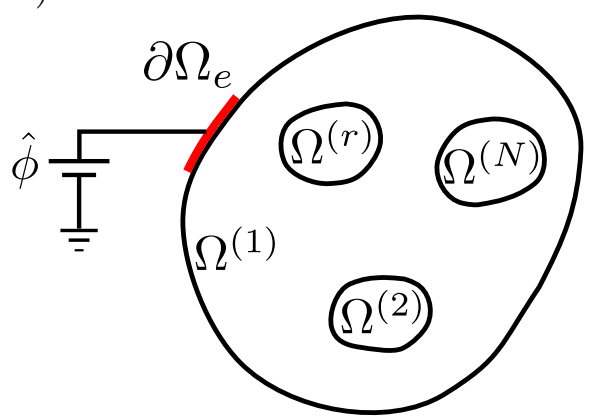

Figura 6.1: Dominio y condiciones de borde para un dieléctrico con varios microdominios $(r=1, \ldots, N)$. Para el caso de especímenes particulados la matriz no contiene cargas libres.

Se define como $\Omega$ al dominio ocupado por el espécimen y como $\Omega^{(r)}(r=1, \ldots, N)$ a los $N$ microdominios que componen al espécimen, de modo tal que $\Omega=\bigcup_{r=1}^{N} \Omega^{(r)}$. Los dominios $\Omega^{(r)}$ son descritos por un conjunto de funciones características $\chi^{(r)}(\mathbf{x})$, que toman el valor 1 si el vector posición $\mathbf{x}$ se encuentra en $\Omega^{(r)}$ y 0 en caso contrario.

\subsubsection{Ecuaciones de campo}

Con respecto a las cargas móviles y siguiendo el trabajo de Trukhan (1963), la densidad volumétrica de carga eléctrica en cualquier punto $\mathbf{x}$ del dominio $\Omega$ se considera como

$$
\rho_{c}(\mathbf{x})=-\nabla \cdot \mathbf{P}(\mathbf{x})+F z\left(c_{+}(\mathbf{x})-c_{-}(\mathbf{x})\right)
$$

donde $c_{+}$y $c_{-}$denotan la concentración molar de cationes móviles y de contraiones fijos con valencia $z$, respectivamente, $\mathbf{P}$ denota el campo vectorial de polarización intrínseca, y $F$ denota la constante de Faraday. Se asume que dentro de cada microdominio $r$ los contraiones fijos se distribuyen uniformemente con concentración molar $c_{-}^{(r)}$. La ley de conservación de cationes dentro de cada microdominio así como la neutralidad eléctrica exigen por lo tanto que

$$
\int_{\Omega^{(r)}}\left(c_{+}(\mathbf{x})-c_{-}^{(r)}\right) \mathrm{d} V=0
$$

para todo $r$. Por otro lado, la densidad superficial de carga eléctrica en las interfaces interiores y en el límite exterior se considera como

$$
\sigma_{c}(\mathbf{x})= \begin{cases}-\llbracket \mathbf{P} \rrbracket \cdot \mathbf{n} & \text { en } \quad \partial \Omega^{(1)}, \ldots, \partial \Omega^{(N)}, \partial \Omega \backslash \partial \Omega_{e} \\ -\llbracket \mathbf{P} \rrbracket \cdot \mathbf{n}+\sigma_{f}(\mathbf{x}) & \text { en } \quad \partial \Omega_{e}\end{cases}
$$


donde $\sigma_{f}(\mathbf{x})$ es una densidad de superficie de cargas libres y $\llbracket \cdot \rrbracket$ representa el salto a través del límite de un dominio con vector normal saliente $\mathbf{n}$.

Las ecuaciones que gobiernan el problema pueden expresarse entonces como - ver, por ejemplo, Xiao y Bhattacharya (2008)-

$$
\left.\begin{array}{rl}
\nabla \cdot \mathbf{D} & =F z\left(c_{+}-c_{-}\right) \\
\mathbf{E} & =-\nabla \phi \\
\nabla \mu_{+} & =\mathbf{0}
\end{array}\right\} \quad \begin{aligned}
\text { en } \quad \mathbb{R}^{3} \backslash \partial \Omega \backslash \partial \Omega^{(1)} \backslash \ldots \backslash \partial \Omega^{(N)}, \\
\text { en } \quad \Omega^{(1)}, \ldots, \Omega^{(N)},
\end{aligned}
$$

con

$$
\mathbf{D}=\left\{\begin{array}{lll}
\epsilon_{0} \mathbf{E} & \text { en } & \mathbb{R}^{3} \backslash \Omega \\
\epsilon_{0} \mathbf{E}+\mathbf{P} & \text { en } & \Omega
\end{array}\right.
$$

y las condiciones de borde

$$
\begin{aligned}
\phi=\hat{\phi} & \text { en } \quad \partial \Omega_{e} \quad \text { con } \quad \phi \rightarrow 0 \quad \text { si } \quad|\mathbf{x}| \rightarrow \infty \\
\llbracket \phi \rrbracket=0 & \text { y } \quad \llbracket \mathbf{D} \rrbracket \cdot \mathbf{n}=0 \quad \text { en } \quad \partial \Omega^{(1)}, \ldots, \partial \Omega^{(N)}, \partial \Omega \backslash \partial \Omega_{e},
\end{aligned}
$$

donde $\phi$ representa nuevamente el potencial electrostático y $\mu_{+}$es el potencial electroquímico de los cationes móviles. Estas expresiones implican que el potencial electrostático es continuo en todo el espacio, mientras que el potencial químico es uniforme de a trozos dentro del sólido, posiblemente tomando distintos valores constantes dentro de cada microdominio. Aquí nuevamente $\mathbf{D}, \mathbf{E}$, y $\mathbf{P}$ son, respectivamente, el desplazamiento eléctrico, la intensidad de campo eléctrico, y la polarizabilidad del material, $\llbracket \cdot \rrbracket$ denota el salto a través de $\partial \Omega$, $\mathbf{n}$ es el vector normal hacia afuera de $\partial \Omega, \epsilon_{0}$ es la permitividad eléctrica del vacío y $\nabla$ es el operador nabla estándar.

\subsubsection{Termodinámica y relaciones constitutivas}

Los argumentos termodinámicos implican entonces que un conjunto amplio de relaciones constitutivas del material están dadas por (veáse, por ejemplo, Xiao y Bhattacharya, 2008)

$$
\mathbf{E}=\frac{\partial \mathfrak{e}}{\partial \mathbf{P}}\left(\mathbf{x}, \mathbf{P}, c_{+}\right), \quad \mathrm{y} \quad \mu_{+}=\frac{\partial \mathfrak{e}}{\partial c_{+}}\left(\mathbf{x}, \mathbf{P}, c_{+}\right)+F z \phi
$$

donde se relaciona la intensidad de campo eléctrico $\mathbf{E}$ y el potencial químico $\mu_{+}$ con la polarización y la concentración molar de iones a través de la energía libre de Helmholtz $\mathfrak{e}$ del espécimen, que caracteriza completamente su respuesta. 
En un espécimen eléctricamente heterogéneo, la función $\mathfrak{e}$ depende explícitamente de $\mathbf{x}$ a través de las funciones características $\chi^{(r)}(\mathbf{x})$ de la siguiente manera

$$
\mathfrak{e}\left(\mathbf{x}, \mathbf{P}, c_{+}\right)=\sum_{r} \chi^{(r)}(\mathbf{x}) \mathfrak{e}^{(r)}\left(\mathbf{P}, c_{+}\right)
$$

donde, en ese caso, la densidad de energía libre que caracteriza cada microdominio $r$ se identificará como $\mathfrak{e}^{(r)}\left(\mathbf{P}, c_{+}\right)$. En este caso y para fines ilustrativos, se considera la una densidad de energía libre con la siguiente forma

$$
\mathfrak{e}^{(r)}\left(\mathbf{P}, c_{+}\right)=\frac{1}{2} \kappa^{(r)} \mathbf{P}^{2}+R T\left(c_{+} \ln \frac{c_{+}}{c_{-}^{(r)}}-\left(c_{+}-c_{-}^{(r)}\right)\right),
$$

donde $\kappa^{(r)}$ es la polarizabilidad intrínseca del microdominio, $R$ es la constante de gas universal, y $T$ es la temperatura absoluta. El primer término corresponde a la energía almacenada en el microdominio por medio de mecanismos de polarización intrínseco, mientras que el segundo término tiene en cuenta la energía de mezcla de los cationes móviles. Cabe mencionar, sin embargo, que esta formulación permite formas generales para la energía $\mathfrak{e}^{(r)}$, en tanto la elección sea de una función estrictamente convexa que satisfaga condiciones de crecimiento adecuadas. Las ecuaciones (6.2)-(6.9) caracterizan completamente la respuesta electrostática del espécimen.

\subsubsection{Formulación Variacional}

Es posible expresar el problema electrostático presentado anteriormente en forma variacional, tomando a los potenciales electrostáticos y químicos como variables primarias. Para ello, en primer lugar se invierten las relaciones constitutivas (6.9) de modo que

$$
\mathbf{P}=\frac{\partial \mathfrak{e}^{*}}{\partial \mathbf{E}}\left(\mathbf{x}, \mathbf{E}, \mu_{+}-F z \phi\right) \quad \mathrm{y} \quad c_{+}=\frac{\partial \mathfrak{e}^{*}}{\partial \mu}\left(\mathbf{x}, \mathbf{E}, \mu_{+}-F z \phi\right)
$$

donde $\mathfrak{e}^{*}$ es una densidad de energía complementaria definida por la transformada de Legendre-Fenchel con respecto a $\mathbf{P}$ y $c$ como

$$
\mathfrak{e}^{*}(\mathbf{x}, \mathbf{E}, \mu)=\sup _{\mathbf{P}, c}[\mathbf{E} \cdot \mathbf{P}+\mu c-\mathfrak{e}(\mathbf{x}, \mathbf{P}, c)]
$$

Es posible entonces definir una densidad de energía complementaria del espécimen y de la porción del campo eléctrico superpuesto como

$$
\psi(\mathbf{x}, \mathbf{E}, \mu) \doteq \frac{1}{2} \epsilon_{0} \mathbf{E}^{2}+\mathfrak{e}^{*}(\mathbf{x}, \mathbf{E}, \mu)-\mu c_{-}(\mathbf{x})
$$


por lo que las relaciones constitutivas en el dominio $\Omega$ pueden entonces reescribirse como

$$
\mathbf{D}=\frac{\partial \psi}{\partial \mathbf{E}}\left(\mathbf{x}, \mathbf{E}, \mu_{+}-F z \phi\right), \quad c_{+}-c_{-}=\frac{\partial \psi}{\partial \mu}\left(\mathbf{x}, \mathbf{E}, \mu_{+}-F z \phi\right)
$$

Dado que la densidad de energía libre es estrictamente convexa, la densidad de energía complementaria también lo es. Resulta entonces de los argumentos estándar del análisis convexo (por ejemplo, Hill, 1956) que los potenciales electrostáticos y químicos surgen del principio mínimo

$$
\mathcal{U}=\min _{\phi(\mathbf{x}) \in \Phi(\hat{\phi})} \min _{\mu_{+}(\mathbf{x}) \in \mathcal{M}} \int_{\Omega} \psi\left(\mathbf{x},-\nabla \phi, \mu_{+}-F z \phi\right) \mathrm{d} V+\int_{\mathbb{R}^{3} \backslash \Omega} \frac{1}{2} \epsilon_{0}|\nabla \phi|^{2} \mathrm{~d} V
$$

donde el conjunto de los potenciales químicos y eléctricos admisibles están definidos como

$$
\begin{aligned}
\Phi(\hat{\phi}) & =\left\{\phi(\mathbf{x}) \in H_{0}^{1}\left(\mathbb{R}^{3}\right) \mid \phi(\mathbf{x})=\hat{\phi}(\mathbf{x}) \text { en } \partial \Omega_{e}\right\} \quad \mathrm{y} \\
\mathcal{M} & =\left\{\mu_{+}(\mathbf{x}) \in L^{\infty}(\Omega) \mid \mu_{+}(\mathbf{x})=\mu_{+}^{(r)} \text { en } \Omega^{(r)}, r=1, \ldots, N\right\}
\end{aligned}
$$

Esta es la formulación variacional del problema electrostático de la sección 6.2.2. En vistas de la convexidad estricta de $\psi$, los campos minimizadores son únicos. Nótese también que dentro de cada microdominio, el potencial químico es uniforme y juego el rol de un multiplicador de Lagrange asociado con la restricción (6.2) que dicta la conservación de cationes.

Además, debe notarse que el funcional en (6.16) depende del potencial electrostático y de su gradiente. Es entonces esperable que aparezcan longitudes características intrínsecas del material. Esto se confirma si se considera la densidad de energía libre propuesta en (6.11), para la cual la densidad de energía complementaria correspondiente $u^{(r)}$ está dada por

$$
u^{(r)}(\mathbf{E}, \mu)=\frac{1}{2} \epsilon^{(r)} \mathbf{E}^{2}+R T c_{-}^{(r)}\left[\exp \left(\frac{\mu}{R T}\right)-\frac{\mu}{R T}-1\right]
$$

donde $\epsilon^{(r)}=\epsilon_{0}+\left(\kappa^{(r)}\right)^{-1}$ es la permitividad del microdominio. Evaluando $u^{(r)}$ en los campos admisibles de (6.16) se obtiene

$$
\begin{array}{r}
u^{(r)}\left(-\nabla \phi, \mu_{+}-F z \phi\right)=R T c_{-}^{(r)}\left[\frac{1}{2} \ell^{(r)^{2}}\left|\frac{F z \nabla \phi}{R T}\right|^{2}+\exp \left(\frac{\mu_{+}-F z \phi}{R T}\right)-\right. \\
\left.-\frac{\mu_{+}-F z \phi}{R T}-1\right]
\end{array}
$$


donde $\ell^{(r)}=\sqrt{R T \epsilon^{(r)} / F^{2} z^{2} c_{-}^{(r)}}$ es la denominada longitud de Debye del microdominio. La longitud de Debye de materiales semiconductores puede variar desde unos pocos nanómetros a unos pocos micrómetros.

\subsection{Formulación multiescala}

\subsubsection{Formulación primaria}

En el límite de microestructura fina, es natural esperar que los especímenes considerados aquí se comporten como medios homogéneos eléctricamente neutros con un único mecanismo de polarización sensible a los gradientes de potencial electrostático. Con base en esta suposición y asumiendo por simplicidad que el espécimen posee una microestructura periódica, se espera que el principio del mínimo (6.16) tome la forma

$$
\mathcal{U}=\min _{\bar{\phi}(\mathbf{x}) \in \Phi(\hat{\phi})} \int_{\Omega} \bar{u}(-\nabla \bar{\phi}) \mathrm{d} V+\int_{\mathbb{R}^{3} \backslash \Omega} \frac{1}{2} \epsilon_{0}|\nabla \bar{\phi}|^{2} \mathrm{~d} V
$$

donde $\bar{\phi}$ es el potencial electrostático macroscópico y $\bar{u}$ es una densidad de energía efectiva complementaria definida por

$$
\bar{u}(\overline{\mathbf{E}})=\min _{\phi(\mathbf{y}) \in \mathcal{K}(\overline{\mathbf{E}})} \min _{\mu_{+}(\mathbf{y}) \in \mathcal{N}}\left\langle u\left(\mathbf{y},-\nabla \phi, \mu_{+}-F z \phi\right)\right\rangle .
$$

En esta expresión, las llaves $\langle\cdot\rangle$ denotan nuevamente el promedio volumétrico en una celda unitaria microestructural $\Omega_{\#}$ que contiene $n$ microdominios $\Omega_{\#}^{(r)}$ - tales que $\Omega_{\#}=\cup_{r=1}^{n} \Omega_{\#}^{(r)}$ - y el conjunto de los campos de potenciales eléctricos y químicos microscópicos admisibles están definidos como

$$
\begin{aligned}
\mathcal{K}(\overline{\mathbf{E}}) & =\left\{\phi(\mathbf{y}) \in H^{1}\left(\Omega_{\#}\right) \mid \phi(\mathbf{y})=-\overline{\mathbf{E}} \cdot \mathbf{y}+\phi_{\#}(\mathbf{y}), \phi_{\#}(\mathbf{y}) \Omega_{\#}-\text { periódico }\right\} \\
\mathcal{N} & =\left\{\mu_{+}(\mathbf{y}) \in L^{\infty}\left(\Omega_{\#}\right) \mid \mu_{+}(\mathbf{y})=\mu_{+}^{(r)} \text { en } \Omega_{\#}^{(r)}, r=1, \ldots, n\right\}
\end{aligned}
$$

Esta expectativa se adopta en base a propuestas exitosas realizadas previamente en el contexto de otros fenómenos físicos con descripciones matemáticas similares. Son particularmente relevantes las formulaciones de Talbot y Willis (1986) para difusión en estado estacionario a través de medios con pérdidas y las formulaciones de Fleck y Willis (2004) para compuestos con plasticidad de gradiente de deformación. Esta última formulación ha sido puesta dentro de un riguroso marco de homogeneización por Francfort et al. (2013) utilizando convergencia $H$; este marco se restringe a energías cuadráticas pero permite microestructuras no periódicas. Una motivación adicional detrás de la presente formulación proviene de las ecuaciones de campo de 
dos escalas que deducen de las condiciones de minimización en (6.21) y (6.22).

En efecto, las condiciones de minimización en (6.21) implican las ecuaciones de campo macroscópicas

$$
\begin{aligned}
& \nabla \cdot \overline{\mathbf{D}}=0 \quad \text { y } \quad \overline{\mathbf{E}}=-\nabla \bar{\phi} \quad \text { en } \quad \mathbb{R}^{3} \backslash \partial \Omega, \\
& \bar{\phi}=\hat{\phi} \quad \text { en } \quad \partial \Omega_{e}, \quad \llbracket \overline{\mathbf{D}} \rrbracket \cdot \mathbf{n}=0 \quad \text { en } \quad \partial \Omega \backslash \partial \Omega_{e}, \\
& \bar{\phi} \rightarrow 0 \quad \text { si } \quad|\mathbf{x}| \rightarrow \infty
\end{aligned}
$$

donde $\bar{\phi}$ es el potencial electrostático macroscópico y $\overline{\mathbf{D}}$ es el desplazamiento eléctrico macroscópico dado por

$$
\overline{\mathbf{D}}= \begin{cases}\frac{\partial \bar{u}}{\partial \overline{\mathbf{E}}}(\overline{\mathbf{E}}) & \text { in } \Omega \\ \epsilon_{0} \overline{\mathbf{E}} & \text { in } \mathbb{R}^{3} \backslash \Omega .\end{cases}
$$

A su vez, las condiciones de minimización en (6.22) implican las ecuaciones de campo microscópicas

$$
\begin{aligned}
& \left.\begin{array}{rl}
\nabla \cdot \mathbf{D} & =F z\left(c_{+}-c_{-}\right), \\
\mathbf{E} & =-\nabla \phi, \\
\phi & =-\overline{\mathbf{E}} \cdot \mathbf{y}+\phi_{\#}
\end{array}\right\} \quad \text { en } \quad \Omega_{\#} \backslash \partial \Omega_{\#}^{(1)} \backslash \ldots \backslash \partial \Omega_{\#}^{(n)}, \\
& \left.\begin{array}{rl}
\mathbf{D} & =\frac{\partial u^{(r)}}{\partial \mathbf{E}}\left(\mathbf{E}, \mu_{+}^{(r)}-F z \phi\right), \\
\left.c_{-}\right) & =\frac{\partial u^{(r)}}{\partial \mu}\left(\mathbf{E}, \mu_{+}^{(r)}-F z \phi\right)
\end{array}\right\} \quad \text { en } \quad \Omega_{\#}^{(r)}, \\
& \left.\begin{array}{rl}
\llbracket \phi \rrbracket & =0, \\
\llbracket \mathbf{D} \rrbracket \cdot \mathbf{n} & =0
\end{array}\right\} \quad \text { en } \quad \partial \Omega_{\#}^{(1)}, \ldots, \partial \Omega_{\#}^{(n)}, \\
& \left\langle c_{+}-c_{-}\right\rangle^{(r)}=0 \text {, }
\end{aligned}
$$

donde $\phi_{\#}$ es periódico en $\Omega_{\#}$, los $\mu_{+}^{(r)}$ 's son uniformes, y $\langle\cdot\rangle^{(r)}$ denota el promedio volumétrico en el microdominio $\Omega_{\#}^{(r)}$. Debe notarse que los potenciales químicos y electrostáticos microscópicos son únicos hasta una constante aditiva global, es decir, si los campos $\left\{F z \phi(\mathbf{y}), \mu_{+}(\mathbf{y})\right\}$ son minimizadores, los campos $\left\{F z \phi(\mathbf{y})+C, \mu_{+}(\mathbf{y})+C\right\}$ también lo son para cualquier constante $C$. Sin embargo, las distribuciones de intensidad de campo eléctrico, desplazamiento eléctrico y concentración de iones correspondientes son únicas.

La expresión (6.28) constituye la relación constitutiva efectiva para el sistema formado por el espécimen y el campo eléctrico. La misma relaciona la intensidad de campo eléctrico con el desplazamiento eléctrico en un punto determinado del espécimen. A 
su vez, estas variables de campo macroscópicas se relacionan con las variables de campo microscópicas correspondientes a través de ciertos promedios volumétricos sobre la celda unitaria microestructural. Efectivamente, la definición del conjunto $\mathcal{K}$ admisible implica la conocida relación de dos escalas

$$
\overline{\mathbf{E}}=\langle\mathbf{E}\rangle
$$

entre las intensidades de campo eléctrico, y la relación constitutiva efectiva (6.28) implica la relación de dos escalas

$$
\overline{\mathbf{D}}=\left\langle\frac{\partial u}{\partial \mathbf{E}}+\frac{\partial u}{\partial \mu} F z \mathbf{y}\right\rangle=\left\langle\mathbf{D}+F z\left(c_{+}-c_{-}\right) \mathbf{y}\right\rangle
$$

entre los desplazamientos eléctricos. Además, una vez que se identifica la diferencia $\overline{\mathbf{D}}-\epsilon_{0} \overline{\mathbf{E}}$ con el vector de polarización macroscópica $\overline{\mathbf{P}}$, las relaciones (6.33) y (6.28) implican la relación de dos escalas

$$
\overline{\mathbf{P}}=\left\langle\mathbf{P}+F z\left(c_{+}-c_{-}\right) \mathbf{y}\right\rangle
$$

entre las polarizaciones. Esto significa que la descripción multiescala anterior relaciona la intensidad de campo eléctrico macroscópica con el promedio volumétrico de la intensidad de campo eléctrico microscópica, pero relaciona la polarización macroscópica (o el desplazamiento eléctrico macroscópico) con el promedio volumétrico de la polarización intrínseca microscópica (o el desplazamiento eléctrico microscópico) más la polarización de cargas espaciales generadas por los cationes desplazados y los contraiones fijos. Por otro lado, no existe un potencial químico macroscópico porque la concentración total de iones siempre se conserva. En efecto esta descripción multiescala es totalmente compatible con la condición de Hill-Mandel, la cual requiere un balance entre la potencia interna macroscópica y microscópica. La potencia interna macroscópica de un sistema compuesto por el espécimen y el campo eléctrico está dada por

$$
\overline{\mathcal{P}}=\int_{\mathbb{R}^{3}} \overline{\mathbf{E}} \cdot \dot{\overline{\mathbf{D}}} \mathrm{d} V
$$

El integrando representa la densidad de potencia macroscópica. Por otro lado, el promedio de la potencia microscópica dentro de la región $\Omega$ está dada por

$$
\mathcal{P}=\left\langle\mathbf{E} \cdot \dot{\mathbf{D}}-\phi F z \dot{c}_{+}+\mu_{+} \dot{c}_{+}\right\rangle
$$

Ahora bien, el promedio del potencial químico $\left\langle\mu_{+} \dot{c}_{+}\right\rangle$es nulo en vistas de (6.32), y 
teniendo en cuenta las ecuaciones de campo microscópicas (6.29)-(6.31) se tiene que

$$
\begin{aligned}
\mathcal{P} & =\left\langle\left(\overline{\mathbf{E}}+\mathbf{E}_{\#}\right) \cdot \dot{\mathbf{D}}-\left(-\overline{\mathbf{E}} \cdot \mathbf{y}+\phi_{\#}\right) F z \dot{c}_{+}\right\rangle \\
& =\overline{\mathbf{E}} \cdot\left\langle\dot{\mathbf{D}}+F z\left(\dot{c}_{+}-\dot{c}_{-}\right) \mathbf{y}\right\rangle+\left\langle\mathbf{E}_{\#} \cdot \dot{\mathbf{D}}-\phi_{\#} F z \dot{c}_{+}\right\rangle \\
& =\overline{\mathbf{E}} \cdot \dot{\overline{\mathbf{D}}}+\left\langle\mathbf{E}_{\#} \cdot \dot{\mathbf{D}}-\phi_{\#} \nabla \cdot \dot{\mathbf{D}}\right\rangle \\
& =\overline{\mathbf{E}} \cdot \dot{\overline{\mathbf{D}}}+\left\langle\left(\mathbf{E}_{\#}+\nabla \phi_{\#}\right) \cdot \dot{\mathbf{D}}\right\rangle-\frac{1}{\left|\Omega_{\#}\right|} \int_{\partial \Omega_{\#}} \phi_{\#} \dot{\mathbf{D}} \cdot \mathbf{n} \mathrm{d} S .
\end{aligned}
$$

El segundo y tercer término son nulos dado que $\mathbf{E}_{\#}=-\nabla \phi_{\#}, \phi_{\#}$ es periódico en $\Omega_{\#}$ y $\dot{\mathbf{D}} \cdot \mathbf{n}$ es antiperiódico $\Omega_{\#}$. Por lo tanto, puede concluirse que la densidad de potencia macroscópica es igual al promedio de la potencia microscópica como es requerido por la condición de Hill-Mandel. A su vez, esto implica que la formulación multiescala provista puede también derivarse invocando el principio multiescala de la potencia virtual de Blanco et al. (2016) junto con las reglas de homogeneización cinemáticas (6.33) y (6.35).

\subsubsection{Formulación dual}

La relación constitutiva efectiva (6.28) puede escribirse inversamente como

$$
\overline{\mathbf{E}}= \begin{cases}\frac{\partial \bar{u}^{*}}{\partial \overline{\mathbf{D}}}(\overline{\mathbf{D}}) & \text { en } \Omega \\ \epsilon_{0}^{-1} \overline{\mathbf{D}} & \text { en } \mathbb{R}^{3} \backslash \Omega .\end{cases}
$$

donde

$$
\bar{u}^{*}(\overline{\mathbf{D}})=\sup _{\overline{\mathbf{E}}}[\overline{\mathbf{D}} \cdot \overline{\mathbf{E}}-\bar{u}(\overline{\mathbf{E}})]
$$

es la transformada de Legendre de $\bar{u}$. Esta función puede escribirse como

$$
\bar{u}^{*}(\overline{\mathbf{D}})=\min _{(\mathbf{D}(\mathbf{y}), c(\mathbf{y})) \in \mathcal{S}(\overline{\mathbf{D}})}\left\langle u^{*}(\mathbf{y}, \mathbf{D}, c)\right\rangle
$$

donde

$$
u^{*}(\mathbf{y}, \mathbf{D}, c)=\sup _{\mathbf{E}, \mu}[\mathbf{D} \cdot \mathbf{E}+c \mu-u(\mathbf{E}, \mu)]
$$

es la transformada de Legendre de $u, \mathrm{y}$

$$
\begin{array}{r}
\mathcal{S}(\overline{\mathbf{D}})=\left\{(\mathbf{D}, c) \mid \nabla \cdot \mathbf{D}=F z c \text { en } \Omega_{\#},\langle c\rangle^{(r)}=0 \text { en } \Omega_{\#}^{(r)}, r=1, \ldots, n,\right. \\
\left.\mathbf{D} \cdot \mathbf{n} \Omega_{\#}-\text { antiperiódica, } \overline{\mathbf{D}}=\langle\mathbf{D}+F z c \mathbf{y}\rangle\right\}
\end{array}
$$


es el conjunto de campos de desplazamiento eléctrico y exceso de cargas admisibles. En esta última expresión, la condición de antiperiodicidad significa que la proyección normal $\mathbf{D} \cdot \mathbf{n}$ debería tener valores opuestos en puntos opuestos del borde $\partial \Omega_{\#}$.

\subsection{Cotas y estimaciones para la densidad de energía específica}

En general, el cálculo de la energía efectiva (6.22) o (6.41) requiere el uso de técnicas numéricas de campo completo que sean capaces de resolver las ecuaciones de EulerLagrange subyacentes. La complejidad matemática de estas ecuaciones surge de la distribución intrincada de interfaces materiales junto con la variación espacial y la no linealidad de la respuesta local. En la práctica, sin embargo, ese nivel de precisión muchas veces no es necesario y el uso de estimaciones aproximadas es suficiente. En esta sección se derivan un conjunto de estimaciones simples basadas en cotas elementales del tipo Voigt y Reuss, así como un conjunto de estimaciones refinadas basadas en el concepto de medio lineal de comparación.

\subsubsection{Cotas de tipo Voigt}

La energía efectiva $\bar{u}$ puede ser acotada por arriba evaluando el potencial (6.22) en un miembro particular del conjunto $\mathcal{K}(\overline{\mathbf{E}})$. La opción más simple $\phi(\mathbf{y})=-\overline{\mathbf{E}} \cdot \mathbf{y}$ da como resultado las cotas superiores de tipo Voigt

$$
\bar{u}(\overline{\mathbf{E}}) \leq \bar{u}_{V}(\overline{\mathbf{E}})=\sum_{r=1}^{n} f^{(r)} \min _{\mu_{+}^{(r)}}\left\langle u^{(r)}\left(\overline{\mathbf{E}}, \mu_{+}^{(r)}+F z \overline{\mathbf{E}} \cdot \mathbf{y}\right)\right\rangle^{(r)}
$$

donde $f^{(r)}=\left|\Omega_{\#}^{(r)}\right| /\left|\Omega_{\#}\right|$ denota la fracción volumétrica de la celda unitaria $\Omega_{\#}$ ocupada por el microdominio $\Omega_{\#}^{(r)}$. La estimación correspondiente para la respuesta efectiva del sólido dieléctrico está dada por

$$
\begin{array}{r}
\overline{\mathbf{D}}=\frac{\partial \bar{u}_{V}}{\partial \overline{\mathbf{E}}}(\overline{\mathbf{E}})=\sum_{r=1}^{n} f^{(r)}\left\langle\frac{\partial u^{(r)}}{\partial \mathbf{E}}\left(\overline{\mathbf{E}}, \mu_{+}^{(r)}+F z \overline{\mathbf{E}} \cdot \mathbf{y}\right)+\right. \\
\left.F z \frac{\partial u^{(r)}}{\partial \mu}\left(\overline{\mathbf{E}}, \mu_{+}^{(r)}+F z \overline{\mathbf{E}} \cdot \mathbf{y}\right) \mathbf{y}\right\rangle^{(r)}
\end{array}
$$

donde los $\mu_{+}^{(r)}$ corresponden a los potenciales químicos que minimizan el problema. Cabe resaltar que las cotas elementales no solo dependen de las fracciones volumétricas, sino también de la forma de los microdominios. 


\subsubsection{Cota de tipo Reuss}

De manera similar, la energía efectiva (6.22) puede ser acotada por debajo evaluando el funcional dual (6.41) en un miembro particular del conjunto $\mathcal{S}(\overline{\mathbf{D}})$ y tomando la transformada de Legendre del potencial resultante $\bar{u}^{*}$. Una cota inferior del tipo Reuss se obtiene a partir de la opción más sencilla $\mathbf{D}(\mathbf{y})=\overline{\mathbf{D}}$ y $c(\mathbf{y})=0$, y está dada por

$$
\bar{u}(\overline{\mathbf{E}}) \geq \bar{u}_{R}(\overline{\mathbf{E}})=\operatorname{mín}_{\substack{\mathbf{E}^{(r)}, \mu^{(r)}: \\ \sum_{r=1}^{n} f^{(r)} \mathbf{E}^{(r)}=\overline{\mathbf{E}}}} \sum_{r=1}^{n} f^{(r)} u^{(r)}\left(\mathbf{E}^{(r)}, \mu^{(r)}\right) .
$$

La estimación correspondiente para la respuesta efectiva del dieléctrico sólido está dada por

$$
\overline{\mathbf{D}}=\frac{\partial \bar{u}_{R}}{\partial \overline{\mathbf{E}}}(\overline{\mathbf{E}})=\sum_{r=1}^{n} f^{(r)} \frac{\partial u^{(r)}}{\partial \mathbf{E}}\left(\mathbf{E}^{(r)}, \mu^{(r)}\right)
$$

donde $\mathbf{E}^{(r)}$ y $\mu^{(r)}$ son los que minimizan (6.46). En vistas del campo de prueba $c(\mathbf{y})=0$, esta cota elemental no tiene en cuenta la presencia de polarización debido a cargas espaciales.

\subsubsection{Estimaciones basadas en medios lineales de comparación}

Es posible obtener estimaciones refinadas que mejoran las cotas anteriores utilizando métodos variacionales basados en el concepto de funcionales de comparación (Talbot y Willis, 1985). La idea principal detrás de estos métodos es aproximar problemas variacionales como el planteado en (6.22) mediante la selección óptima de problemas de comparación que son más sencillos de resolver. En este trabajo se utiliza un método propuesto recientemente por Ponte Castañeda (2016), el cual genera estimaciones para problemas variacionales no lineales en términos de problemas lineales de comparación. Para facilitar la presentación, se centra la atención en problemas cuya densidad de energía complementaria tienen la forma

$$
u^{(r)}(\mathbf{E}, \mu)=\frac{1}{2} \epsilon^{(r)} \mathbf{E}^{2}+g^{(r)}(\mu)
$$

con $g^{(r)}$ convexo; esta forma incluye energías de la forma (6.19). Otros tipos de energías más generales con dependencias no cuadráticas en ambas variables pueden manejarse de manera semejante.

En vistas de la forma (6.48) para los potenciales locales, las estimaciones lineales de comparación pueden construirse introduciendo potenciales de comparación de la 
forma

$$
u_{0}^{(r)}\left(\mathbf{E}, \mu ; \alpha_{0}^{(r)}, \beta_{0}^{(r)}\right)=\frac{1}{2} \epsilon^{(r)} \mathbf{E}^{2}+g_{0}^{(r)}\left(\mu ; \alpha_{0}^{(r)}, \beta_{0}^{(r)}\right)
$$

con

$$
g_{0}^{(r)}\left(\mu ; \alpha_{0}^{(r)}, \beta_{0}^{(r)}\right)=\frac{1}{2} \alpha_{0}^{(r)} \mu^{2}+\beta_{0}^{(r)} \mu
$$

y definiendo las funciones desplazadas

$$
v^{(r)}\left(\alpha_{0}^{(r)}, \beta_{0}^{(r)}\right)=\operatorname{stat}_{\mu}\left[g^{(r)}(\mu)-g_{0}^{(r)}\left(\mu ; \alpha_{0}^{(r)}, \beta_{0}^{(r)}\right)\right]
$$

donde la operación estacionaria (stat) consiste en igualar a cero a las derivadas del argumento con respecto a la variable. El término lineal en el potencial de comparación $g_{0}^{(r)}$ es esencial en vistas de las condiciones de crecimiento no estándar que exhiben los potenciales no lineales $g^{(r)}$. En general, la función $g^{(r)}-g_{0}^{(r)}$ tendrá dos puntos estacionarios como se muestra en la figura 6.2a y, por lo tanto, la función desplazada $v^{(r)}$ tendrá bifurcaciones. Si se considera que $\breve{\mu}^{(r)}$ y $\hat{\mu}^{(r)}$ denotan los dos valores óptimos de $\mu$ para las propiedades de comparación $\alpha_{0}^{(r)}$ y $\beta_{0}^{(r)}$, y $\breve{v}^{(r)}$ y $\hat{v}^{(r)}$ son los valores correspondientes de $v^{(r)}$, entonces siguiendo a Ponte Castañeda (2016) las estimaciones lineales de comparación para la densidad de energía complementaria efectiva están dadas por

$$
\begin{array}{r}
\bar{u}(\overline{\mathbf{E}}) \approx \bar{u}_{L C}(\overline{\mathbf{E}})=\operatorname{stat}_{\substack{\alpha_{0}^{(r)}>0 \\
\beta_{0}^{(r)}}}\left[\bar{u}_{0}\left(\overline{\mathbf{E}} ; \alpha_{0}^{(r)}, \beta_{0}^{(r)}\right)+\frac{1}{2} \sum_{r=1}^{n} f^{(r)}\left(\hat{v}^{(r)}\left(\alpha_{0}^{(r)}, \beta_{0}^{(r)}\right)+\right.\right. \\
\left.\left.+\breve{v}^{(r)}\left(\alpha_{0}^{(r)}, \beta_{0}^{(r)}\right)\right)\right],
\end{array}
$$

donde $\bar{u}_{0}$ es la energía efectiva del medio lineal de comparación definido por

$$
\bar{u}_{0}\left(\overline{\mathbf{E}} ; \alpha_{0}^{(r)}, \beta_{0}^{(r)}\right)=\operatorname{mín}_{\phi(\mathbf{y}) \in \mathcal{K}(\overline{\mathbf{E}})} \min _{\mu_{+}^{(r)}} \sum_{r=1}^{n} f^{(r)}\left\langle u_{0}^{(r)}\left(-\nabla \phi, \mu_{+}^{(r)}-F z \phi\right)\right\rangle^{(r)} .
$$

La minimización con respecto a los potenciales químicos en esta expresión puede realizarse de manera explícita, obteniéndose

$$
\begin{aligned}
\bar{u}_{0}\left(\overline{\mathbf{E}} ; \alpha_{0}^{(r)}, \beta_{0}^{(r)}\right)=\min _{\phi(\mathbf{y}) \in \mathcal{K}(\overline{\mathbf{E}})} \sum_{r=1}^{n} f^{(r)}\left[\frac{1}{2} \epsilon^{(r)}\left\langle|\nabla \phi|^{2}\right\rangle^{(r)}\right. & +\frac{1}{2} \alpha_{0}^{(r)} F^{2} z^{2}\left(\left\langle\phi^{2}\right\rangle^{(r)}-\right. \\
& \left.\left.-\langle\phi\rangle^{(r)^{2}}\right)-\frac{1}{2} \frac{\beta_{0}^{(r)^{2}}}{\alpha_{0}^{(r)}}\right] .
\end{aligned}
$$

Además, en vistas de la linealidad de las ecuaciones de Euler-Lagrange asociadas, 
a)

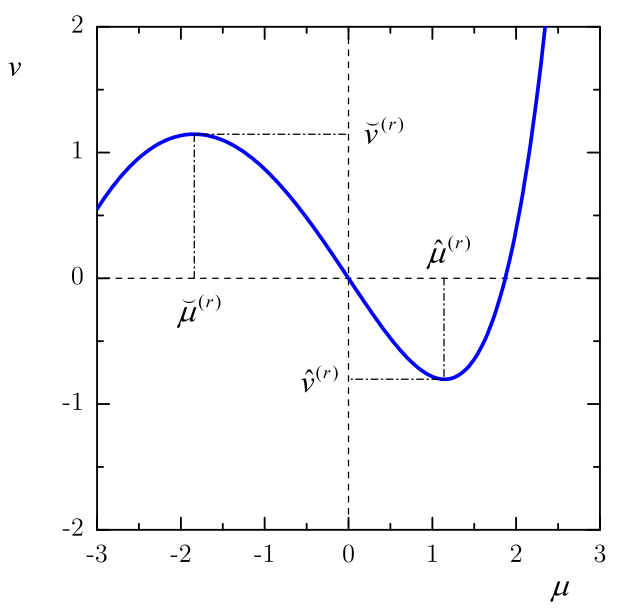

b)

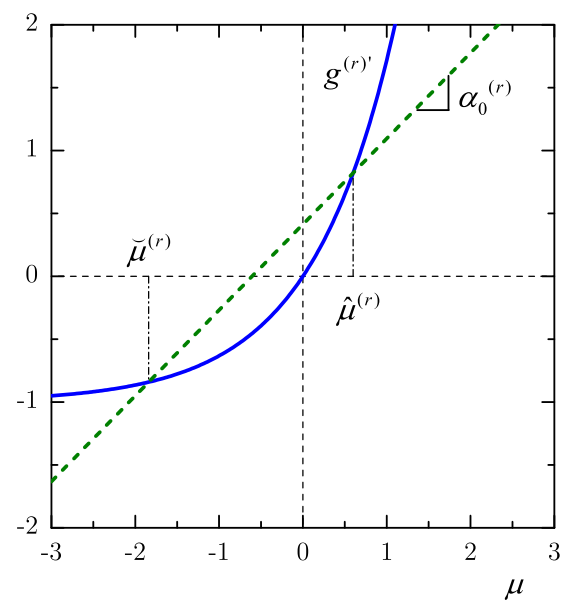

Figura 6.2: Linealización esquemática de la función $g^{(r)}(\mu)=\exp (\mu)-\mu-1$ : a) función desplazada en la expresión (6.51) para la condición $\alpha_{0}^{(r)}=\beta_{0}^{(r)}=1$; b) condición secante generalizada.

esta densidad de energía efectiva de comparación puede ser escrita como

$$
\bar{u}_{0}\left(\overline{\mathbf{E}} ; \alpha_{0}^{(r)}, \beta_{0}^{(r)}\right)=\frac{1}{2} \overline{\mathbf{E}} \cdot \overline{\boldsymbol{\epsilon}}_{0}\left(\alpha_{0}^{(r)}\right) \overline{\mathbf{E}}-\frac{1}{2} \sum_{r=1}^{n} f^{(r)} \frac{\beta_{0}^{(r)^{2}}}{\alpha_{0}^{(r)}},
$$

donde $\overline{\boldsymbol{\epsilon}}_{0}$ es un tensor permitividad efectivo. Entonces, dado cualquier estimación o resultado exacto para la energía efectiva (6.55) del medio lineal de comparación, la expresión (6.52) permite obtener una estimación para la energía efectiva del medio no lineal. Su cálculo requiere resolver un conjunto de $4 n$ ecuaciones algebraicas no lineales para las variables $\breve{\mu}^{(r)}, \hat{\mu}^{(r)}, \alpha_{0}^{(r)}$ y $\beta_{0}^{(r)}$, generadas mediante la condición de estacionaridad. Esta condición con respecto a $\mu$ en las funciones (6.51) genera las ecuaciones

$$
g^{(r)^{\prime}}\left(\hat{\mu}^{(r)}\right)=\alpha_{0}^{(r)} \hat{\mu}^{(r)}+\beta_{0}^{(r)} \quad \text { y } \quad g^{(r)^{\prime}}\left(\breve{\mu}^{(r)}\right)=\alpha_{0}^{(r)} \breve{\mu}^{(r)}+\beta_{0}^{(r)}
$$

mientras que la estacionaridad con respecto a las propiedades lineales de comparación en 6.52 generan las ecuaciones

$$
\frac{\hat{\mu}^{(r)^{2}}+\breve{\mu}^{(r)^{2}}}{2}=\frac{2}{f^{(r)}} \frac{\partial \bar{u}_{0}}{\partial \alpha_{0}^{(r)}} \quad \text { y } \quad \frac{\hat{\mu}^{(r)}+\breve{\mu}^{(r)}}{2}=\frac{1}{f^{(r)}} \frac{\partial \bar{u}_{0}}{\partial \beta_{0}^{(r)}} .
$$

En general, estas ecuaciones deben resolverse numéricamente.

Cabe resaltar que las ecuaciones (6.56) implican que

$$
g^{(r)^{\prime}}\left(\hat{\mu}^{(r)}\right)-g^{(r)^{\prime}}\left(\breve{\mu}^{(r)}\right)=\alpha_{0}^{(r)}\left(\hat{\mu}^{(r)}-\breve{\mu}^{(r)}\right)
$$


y que las ecuaciones (6.57), junto con las identidades

$$
\frac{2}{f^{(r)}} \frac{\partial \bar{u}_{0}}{\partial \alpha_{0}^{(r)}}=\left\langle\left(\mu_{+}^{(r)}-F z \phi\right)^{2}\right\rangle^{(r)} \quad \mathrm{y} \quad \frac{1}{f^{(r)}} \frac{\partial \bar{u}_{0}}{\partial \beta_{0}^{(r)}}=\left\langle\left(\mu_{+}^{(r)}-F z \phi\right)\right\rangle^{(r)}
$$

implican que

$$
\begin{aligned}
\left(\frac{\hat{\mu}^{(r)}+\breve{\mu}^{(r)}}{2}\right) & =\mu_{+}^{(r)}-F z\langle\phi\rangle^{(r)} \quad \mathrm{y} \\
\left(\frac{\hat{\mu}^{(r)}-\breve{\mu}^{(r)}}{2}\right)^{2} & =F^{2} z^{2}\left(\left\langle\phi^{2}\right\rangle^{(r)}-\langle\phi\rangle^{(r)^{2}}\right) .
\end{aligned}
$$

En estas últimas expresiones, $\phi$ y $\mu_{+}^{(r)}$ son los potenciales electrostático y químico en el medio de comparación, y las identidades (6.59) surgen de los argumentos estándar de la homogeneización variacional (véase, por ejemplo, Idiart y Castañeda, 2007). Por lo tanto, el medio de comparación lineal óptimo es un sólido dieléctrico con la misma microestructura que el medio no lineal, pero con fases caracterizadas por una linealización secante generalizada de la respuesta electroquímica no lineal, como se muestra en la figura $6.2 \mathrm{~b}$, que depende de manera autoconsistente del promedio de fases y de las fluctuaciones intrafase del potencial electrostático en el medio lineal de comparación. Para el caso de fluctuaciones intrafase muy pequeñas, $\hat{\mu}^{(r)} \rightarrow \breve{\mu}^{(r)}$ y la linealización secante generalizada se reduce a la linealización tangente. Las relaciones (6.58)-(6.61) pueden utilizarse para reducir el sistema de $4 n$ ecuaciones (6.56)-(6.57) a un sistema de $2 n$ ecuaciones para el conjunto de variables $\breve{\mu}^{(r)}$ y $\hat{\mu}^{(r)}$ de la siguiente manera

$$
g^{(r)^{\prime}}\left(\hat{\mu}^{(r)}\right)+g^{(r)^{\prime}}\left(\breve{\mu}^{(r)}\right)=0 \quad \text { y } \quad\left(\frac{\hat{\mu}^{(r)}-\breve{\mu}^{(r)}}{2}\right)^{2}=F^{2} z^{2}\left(\left\langle\phi^{2}\right\rangle^{(r)}-\langle\phi\rangle^{(r)^{2}}\right),
$$

donde las fluctuaciones del campo potencial electrostático surgen de la identidad

$$
F^{2} z^{2}\left(\left\langle\phi^{2}\right\rangle^{(r)}-\langle\phi\rangle^{(r)^{2}}\right)=\frac{1}{f^{(r)}} \overline{\mathbf{E}} \cdot \frac{\partial \overline{\boldsymbol{\epsilon}}_{0}}{\partial \alpha_{0}^{(r)}}\left(\alpha_{0}^{(r)}\right) \overline{\mathbf{E}}
$$

y las propiedades lineales de comparación $\alpha_{0}^{(r)}$ se dan en términos de las variables $\breve{\mu}^{(r)}$ y $\hat{\mu}^{(r)}$ a través de

$$
\alpha_{0}^{(r)}=\frac{g^{(r)^{\prime}}\left(\hat{\mu}^{(r)}\right)-g^{(r)^{\prime}}\left(\breve{\mu}^{(r)}\right)}{\hat{\mu}^{(r)}-\breve{\mu}^{(r)}} .
$$

Entonces, haciendo uso de las diversas condiciones de optimalidad, la expresión (6.52) 
para la densidad de energía efectiva puede simplificarse como

$$
\bar{u}_{L C}(\overline{\mathbf{E}})=\sum_{r=1}^{n} f^{(r)} \frac{1}{2}\left[\epsilon^{(r)} \hat{E}^{(r)^{2}}+g^{(r)}\left(\hat{\mu}^{(r)}\right)+g^{(r)}\left(\breve{\mu}^{(r)}\right)\right]
$$

donde $\hat{E}^{(r)} \doteq \sqrt{\left\langle|\nabla \phi|^{2}\right\rangle^{(r)}}$ es una medida de los segundos momentos del campo eléctrico dentro del microdominio $r$ en el medio lineal de comparación. Nótese que la ecuación (6.62) tiene dos raíces por microdominio en vistas de la potencia cuadrática en $(6.62)_{2}$. Sin embargo, las dos raíces equivalen a intercambiar las variables $\hat{\mu}^{(r)}$ y $\breve{\mu}^{(r)}$, las cuales ingresan en las diversas expresiones de manera simétrica y, por lo tanto, proveen la misma estimación para la densidad de energía específica. Finalmente, la estimación para las relaciones constitutivas efectivas surgen de la diferenciación de la expresión (6.52). Debido a las condiciones de estacionaridad, se puede demostrar fácilmente que

$$
\overline{\mathbf{D}}=\frac{\partial \bar{u}_{L C}}{\partial \overline{\mathbf{E}}}(\overline{\mathbf{E}})=\frac{\partial \bar{u}_{0}}{\partial \overline{\mathbf{E}}}\left(\overline{\mathbf{E}} ; \alpha_{0}^{(r)}, \beta_{0}^{(r)}\right)=\overline{\boldsymbol{\epsilon}}_{0}\left(\alpha_{0}^{(r)}\right) \overline{\mathbf{E}}
$$

donde los $\alpha_{0}^{(r)}$ son las propiedades lineales de comparación óptimas, que dependen de $\overline{\mathbf{E}}$. Por lo tanto, la relación constitutiva no lineal coincide exactamente con la relación constitutiva 'instantánea' del medio lineal de comparación.

\subsection{Especialización a densidades de energía particulares}

\subsubsection{Densidad de energía efectiva exacta}

Si en particular se centra el estudio en densidades de energía complementarias de la forma (6.19), las relaciones constitutivas correspondientes son de la forma

$$
\mathbf{D}=\epsilon^{(r)} \mathbf{E} \quad \mathrm{y} \quad c_{+}=c_{-}^{(r)} \exp \left(\frac{\mu_{+}^{(r)}-F z \phi}{R T}\right) .
$$

En este caso, la densidad de energía efectiva (6.22) está dada por

$$
\begin{array}{r}
\bar{u}(\overline{\mathbf{E}})=\min _{\phi \in \mathcal{K}(\overline{\mathbf{E}})} \min _{\mu_{+}^{(r)}} \sum_{r=1}^{n} f^{(r)}\left\langle\frac{1}{2} \epsilon^{(r)}|\nabla \phi|^{2}+R T c_{-}^{(r)}\left[\exp \left(\frac{\mu_{+}^{(r)}-F z \phi}{R T}\right)-\right.\right. \\
\left.\left.-\frac{\mu_{+}^{(r)}-F z \phi}{R T}-1\right]\right\rangle^{(r)} .
\end{array}
$$


Llevando a cabo la minimización con respecto a los potenciales químicos, se obtiene

$$
\begin{aligned}
\bar{u}(\overline{\mathbf{E}})= & \min _{\phi \in \mathcal{K}(\overline{\mathbf{E}})} \sum_{r=1}^{n} f^{(r)}\left[\frac{1}{2} \epsilon^{(r)}\left\langle|\nabla \phi|^{2}\right\rangle^{(r)}+\right. \\
& \left.+R T c_{-}^{(r)} \ln \left\langle\exp \left(-\frac{F z}{R T}\left(\phi-\langle\phi\rangle^{(r)}\right)\right)\right\rangle^{(r)}\right] .
\end{aligned}
$$

Esta expresión involucra un problema de minimización no lineal con respecto al potencial electrostático. Debe notarse que el segundo término entre corchetes está acotado por debajo en vistas de la concavidad del logaritmo y la desigualdad de Jensen.

Es evidente de la expresión (6.68) que los campos minimizadores son tales que $|\nabla \phi| \rightarrow 0$ y $\left|\mu_{+}^{(r)}-F z \phi\right| \rightarrow 0$ cuando $|\overline{\mathbf{E}}| \rightarrow 0$. Entonces, para estados de carga eléctrica débiles, la densidad de energía efectiva admite una expansión de la forma

$$
\bar{u}(\overline{\mathbf{E}})=\frac{1}{2} \overline{\mathbf{E}} \cdot \overline{\boldsymbol{\epsilon}} \overline{\mathbf{E}}+O\left(|\overline{\mathbf{E}}|^{3}\right),
$$

donde el término de orden principal está dado por

$$
\frac{1}{2} \overline{\mathbf{E}} \cdot \overline{\boldsymbol{\epsilon}} \overline{\mathbf{E}}=\min _{\phi \in \mathcal{K}(\overline{\mathbf{E}})} \min _{\mu_{+}^{(r)}} \sum_{r=1}^{n} f^{(r)}\left\langle\frac{1}{2} \epsilon^{(r)}|\nabla \phi|^{2}+\frac{1}{2} \frac{c_{-}^{(r)}}{R T}\left(\mu_{+}^{(r)}-F z \phi\right)^{2}\right\rangle^{(r)}
$$

Llevando a cabo la minimización con respecto a los potenciales químicos, se obtiene

$$
\begin{aligned}
\frac{1}{2} \overline{\mathbf{E}} \cdot \overline{\boldsymbol{\epsilon}} \overline{\mathbf{E}}= & \min _{\phi \in \mathcal{K}(\overline{\mathbf{E}})} \sum_{r=1}^{n} f^{(r)}\left[\frac{1}{2} \epsilon^{(r)}\left\langle|\nabla \phi|^{2}\right\rangle^{(r)}+\right. \\
& \left.+\frac{1}{2} \frac{F^{2} z^{2} c_{-}^{(r)}}{R T}\left\langle\left(\phi-\langle\phi\rangle^{(r)}\right)^{2}\right\rangle^{(r)}\right]
\end{aligned}
$$

De este modo, la respuesta macroscópica inicial está caracterizada completamente por un tensor permitividad efectivo $\bar{\epsilon}$, cuyo cálculo requiere la resolución de un problema de minimización lineal. 


\subsubsection{Cotas de Voigt}

Cuando las densidades de energía complementarias locales son de la forma (6.19), la cota de Voigt presentada en (6.44) puede escribirse como

$$
\begin{aligned}
\bar{u}_{V}(\overline{\mathbf{E}})=\frac{1}{2}\langle\epsilon\rangle \overline{\mathbf{E}}^{2}+\min _{\mu_{+}^{(r)}} \sum_{r=1}^{n} f^{(r)} R T c_{-}^{(r)}\langle & \exp \left(\frac{\mu_{+}^{(r)}+F z \overline{\mathbf{E}} \cdot \mathbf{y}}{R T}\right)- \\
& \left.-\frac{\mu_{+}^{(r)}+F z \overline{\mathbf{E}} \cdot \mathbf{y}}{R T}-1\right\rangle^{(r)} .
\end{aligned}
$$

donde $\langle\epsilon\rangle=\sum_{r=1}^{n} f^{(r)} \epsilon^{(r)}$ es la permitividad media. La minimización con respecto a los potenciales químicos puede realizarse de manera explícita, por lo que la cota puede entonces escribirse como

$$
\bar{u}_{V}(\overline{\mathbf{E}})=\frac{1}{2}\langle\epsilon\rangle \overline{\mathbf{E}}^{2}+\sum_{r=1}^{n} f^{(r)} R T c_{-}^{(r)} \ln \left\langle\exp \left(\frac{F z}{R T} \overline{\mathbf{E}} \cdot\left(\mathbf{y}-\langle\mathbf{y}\rangle^{(r)}\right)\right)\right\rangle^{(r)}
$$

Para intensidades de campo eléctrico débiles, esta cota admite una expansión de la forma

$$
\bar{u}_{V}(\overline{\mathbf{E}})=\frac{1}{2} \overline{\mathbf{E}} \cdot \overline{\boldsymbol{\epsilon}}_{V} \overline{\mathbf{E}}+O\left(|\overline{\mathbf{E}}|^{3}\right)
$$

donde $\overline{\boldsymbol{\epsilon}}_{V}$ es un tensor permitividad efectivo inicial dado por

$$
\overline{\boldsymbol{\epsilon}}_{V}=\langle\epsilon\rangle \mathbf{I}+\frac{F^{2} z^{2}}{R T} \sum_{r=1}^{n} f^{(r)} c_{-}^{(r)}\left(\langle\mathbf{y} \otimes \mathbf{y}\rangle^{(r)}-\langle\mathbf{y}\rangle^{(r)} \otimes\langle\mathbf{y}\rangle^{(r)}\right) .
$$

Alternativamente, esta expresión puede escribirse como

$$
\overline{\boldsymbol{\epsilon}}_{V}=\sum_{r=1}^{n} f^{(r)} \epsilon^{(r)}\left[\mathbf{I}+\left(\frac{L^{(r)}}{\ell^{(r)}}\right)^{2} \mathbf{S}^{(r)}\right]
$$

donde $L^{(r)}=\left|\Omega_{\#}^{(r)}\right|^{1 / 3}$ y $\ell^{(r)}$ son las longitudes característica y de Debye del microdominio $r$, respectivamente, y los tensores $\mathbf{S}^{(r)}$ son cantidades adimensionales dadas por

$$
\mathbf{S}^{(r)}=\frac{1}{L^{(r)^{2}}}\left(\langle\mathbf{y} \otimes \mathbf{y}\rangle^{(r)}-\langle\mathbf{y}\rangle^{(r)} \otimes\langle\mathbf{y}\rangle^{(r)}\right)
$$

De este modo, la influencia de la polarización por cargas espaciales en la cota de Voigt para la permitividad efectiva ingresa a través de los tensores entre corchetes en (6.77), los cuales dependen de la forma de los microdominios a través de los segundos momentos de las fluctuaciones intrafase del vector posición y. Además, estos tensores 
son definidos positivos en vista de la desigualdad de Hölder. Por lo tanto, de acuerdo a la cota de Voigt, la polarización por cargas espaciales actúa efectivamente como un mecanismo de amplificación de las permitividades locales cuya magnitud depende del tamaño de los microdominios.

Por otro lado, para intensidades de campo eléctrico elevadas, la cota (6.74) tiende a

$$
\bar{u}_{V}(\overline{\mathbf{E}})=\frac{1}{2}\langle\epsilon\rangle \overline{\mathbf{E}}^{2}+\sum_{r=1}^{n} f^{(r)} F z c_{-}^{(r)} \sup _{\mathbf{y} \in \Omega^{(r)}} \overline{\mathbf{E}} \cdot\left(\mathbf{y}-\langle\mathbf{y}\rangle^{(r)}\right) .
$$

El segundo término en esta expresión depende linealmente del campo eléctrico. Entonces, de acuerdo a la cota de Voigt, la contribución de la polarización por cargas espaciales a la polarización total se satura al incrementar la intensidad de campo hasta un valor dado por los dipolos más grandes que puedan ser inducidos a lo largo de una dirección de campo determinada.

\subsubsection{Cota de Reuss}

En el caso de la cota de Reuss, es sencillo verificar que los óptimos $\mu^{(r)}$ son nulos, por lo que la densidad de energía efectiva está dada simplemente por

$$
\bar{u}_{R}(\overline{\mathbf{E}})=\frac{1}{2} \overline{\mathbf{E}} \cdot \overline{\boldsymbol{\epsilon}}_{R} \overline{\mathbf{E}}
$$

donde $\overline{\boldsymbol{\epsilon}}_{R}$ es es la media armónica de las permitividades locales dada por

$$
\overline{\boldsymbol{\epsilon}}_{R}=\left\langle\epsilon^{-1}\right\rangle^{-1} \mathbf{I}
$$

Esta expresión coincide exactamente con la bien conocida cota de Reuss para sólidos dieléctricos sin cargas móviles, como es de esperar por los campos de prueba utilizados en su derivación.

\subsubsection{Estimaciones lineales de comparación}

Cuando las densidades de energía complementarias son de la forma 6.19, el sistema de ecuaciones (6.62) puede reducirse a un sistema de ecuaciones para $n$ longitudes de Debye de comparación definidas como $\ell_{0}^{(r)^{2}}=\epsilon^{(r)} / F^{2} z^{2} \alpha_{0}^{(r)}$. Efectivamente, en este caso las condiciones de optimización (6.62) 1 y (6.64) toman la forma

$$
\begin{gathered}
\frac{\exp \left(\hat{\mu}^{(r)} / R T\right)+\exp \left(\breve{\mu}^{(r)} / R T\right)}{2}=1 \quad \mathrm{y} \\
\alpha_{0}^{(r)}=c_{-}^{(r)} \frac{\exp \left(\hat{\mu}^{(r)} / R T\right)-\exp \left(\breve{\mu}^{(r)} / R T\right)}{\hat{\mu}^{(r)}-\breve{\mu}^{(r)}} .
\end{gathered}
$$


Entonces, introduciendo las variables no dimensionales $m^{(r)}=\left(\hat{\mu}^{(r)}-\breve{\mu}^{(r)}\right) / 2 R T$, estas ecuaciones implican que

$$
\left(\ell_{0}^{(r)} / \ell^{(r)}\right)^{2}=m^{(r)} \operatorname{coth}\left(m^{(r)}\right)
$$

mientras que las identidades $(6.62)_{2}$ y (6.63) implican que

$$
m^{(r)}=\left[\frac{(F z / R T)^{2}}{f^{(r)} \epsilon^{(r)}} \overline{\mathbf{E}} \cdot \frac{\partial \overline{\boldsymbol{\epsilon}}_{0}}{\partial \ell_{0}^{(r)^{-2}}} \overline{\mathbf{E}}\right]^{1 / 2}
$$

En esta última expresión, el término a mano derecha no depende explícitamente de las variables $m^{(r)}$. Si se introduce (6.84) en (6.83) se obtiene entonces un sistema de $n$ ecuaciones no lineales para las variables $\ell_{0}^{(r)}$ que debe ser resuelto, en general, numéricamente. Una vez que se determinan las longitudes de Debye de comparación, la estimación para la respuesta efectiva surge de (6.66). 


\section{Aplicación a sistemas materiales modelo}

\subsection{Introducción}

La teoría presentada en el capítulo anterior para dieléctricos con oclusiones semiconductoras se utiliza aquí para derivar y evaluar estimaciones acerca del comportamiento de distintos sistemas materiales representativos. En particular, se centra la atención en laminados bifásicos simples y compuestos particulados para los cuales se encuentran disponibles trabajos de otros autores que permiten validar los resultados obtenidos.

\subsection{Materiales laminados}

A modo de ejemplo, se considera aquí un sólido dieléctrico cuya microestructura consiste de un arreglo periódico de capas planas de dos fases alternativas $(r=1,2)$, ambas caracterizadas por energías complementarias de la forma (6.19). Todas las capas de una fase $r$ dada son de igual espesor $L^{(r)}$, de modo que la celda unitaria consiste de dos capas y la fracción volumétrica de cada fase está dada por $f^{(r)}=$ $L^{(r)} / L$, donde $L=L^{(1)}+L^{(2)}$ es el espesor de la celda unitaria, como se observa en la figura 7.1a. En estos sistemas materiales, la separación de escalas de longitud aplica solo para campos eléctricos perpendiculares a las capas. Bajo estas condiciones de carga, las ecuaciones de campo son similares a aquellas para un capacitor plano con un dieléctrico semiconductor y electrodos bloqueantes (Macdonald, 1959; Xiao y Bhattacharya, 2001). Inicialmente se considera una respuesta lineal para intensidades de campo $\bar{E}$ muy por debajo de la intensidad característica $E_{0}=R T / F z L$, y luego se considera la respuesta no lineal para campos de intensidad arbitraria.

\subsubsection{Respuesta lineal}

Para intensidades de campo eléctrico bajas, la densidad de energía complementaria efectiva puede ser caracterizada por completo a través de la permitividad efectiva normal $\bar{\epsilon}_{n}$ definida por (6.72). Si se lleva a cabo la minimización con respecto al potencial electrostático y las operaciones de promedio volumétrico se obtiene

$$
\bar{\epsilon}_{n}=\left(\frac{f^{(1)}}{\epsilon_{*}^{(1)}}+\frac{f^{(2)}}{\epsilon_{*}^{(2)}}\right)^{-1}
$$


a)

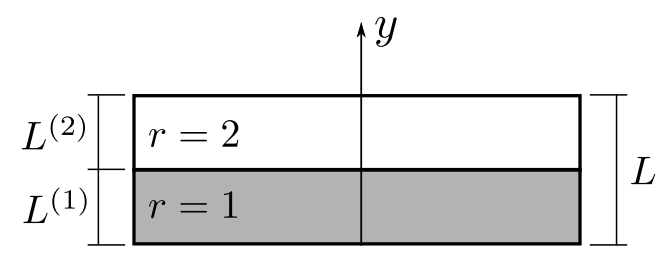

b)

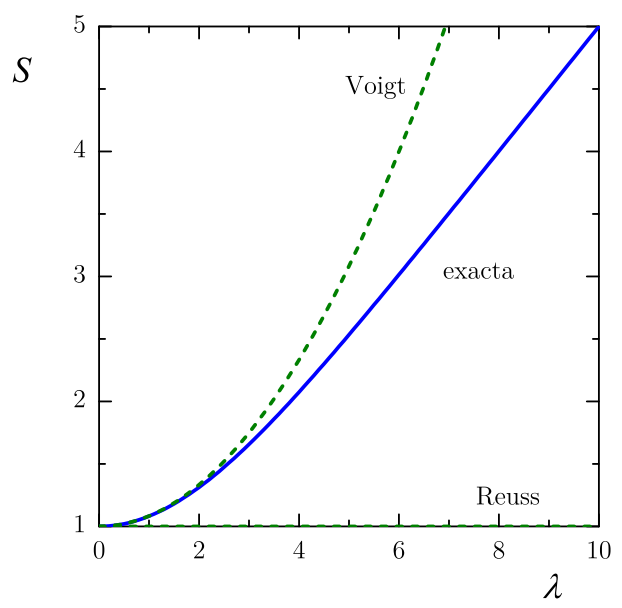

Figura 7.1: Materiales laminados: a) celda unitaria; b) factor de amplificación.

donde $\operatorname{los} \epsilon_{*}^{(r)}$ denotan permitividades equivalentes dadas por

$$
\epsilon_{*}^{(r)}=\epsilon^{(r)} S\left(L^{(r)} / \ell^{(r)}\right) \quad \text { con } \quad S(\lambda)=\frac{\lambda}{2} \operatorname{coth} \frac{\lambda}{2} .
$$

En el Apéndice A.1 pueden encontrarse los detalles de estos cálculos. Entonces, la influencia de la polarización por cargas espaciales en la permitividad efectiva se produce a través de la función $S$ dependiente de la talla y que juega el rol de factor de amplificación de las permitividades locales. La función $S$ se muestra en línea continua en la figura 7.1b. Resulta evidente que si una fase no contiene iones, la longitud de Debye es infinitamente grande por lo que el factor de amplificación vale uno y la fase se comporta como un dieléctrico lineal con permitividad $\epsilon^{(r)}$. Por otro lado, si una fase contiene una gran cantidad de iones, la longitud de Debye tiene entonces una magnitud muy pequeña, por lo que el factor de amplificación es infinitamente grande y la fase se comporta como un conductor perfecto. Finalmente, si una fase tiene una cantidad finita de iones, el factor de amplificación depende de la relación entre el espesor de las capas y la longitud de Debye. Cuanto mayor espesor tengan las capas, mayor será el dipolo de las cargas espaciales y por lo tanto mayor será el factor de amplificación. Por ejemplo, la permitividad equivalente de una capa con iones móviles cuyo espesor es cuatro veces la longitud de Debye, es de aproximadamente el doble que la de la misma capa sin iones. El mismo factor de amplificación puede identificarse en la expresión de Macdonald (1959) para la capacitancia inicial de un capacitor semiconductor.

En la figura 7.2 se presentan predicciones para un medio laminado con $\epsilon^{(1)}=\epsilon^{(2)}=\epsilon$, $\ell^{(1)} \rightarrow \infty \mathrm{y} \ell^{(2)}=\ell$. Esto significa que ambas fases tienen la misma permitividad pero solamente una fase $(r=2)$ contiene iones móviles. Las figuras 7.2a y 7.2b muestran 
a)

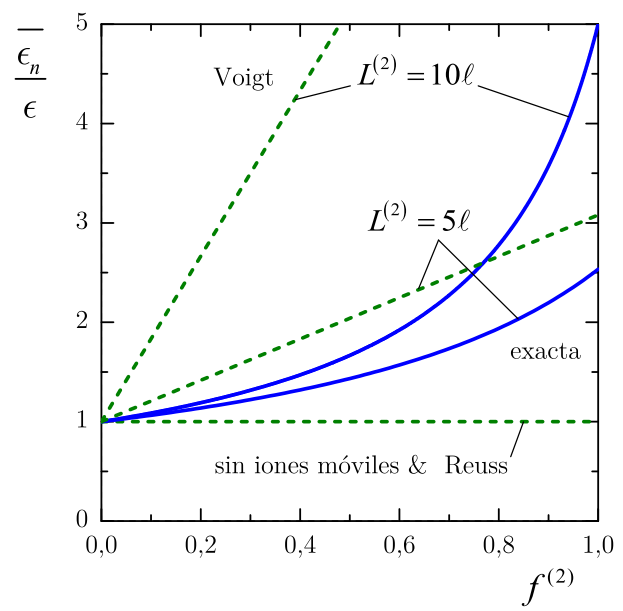

c)

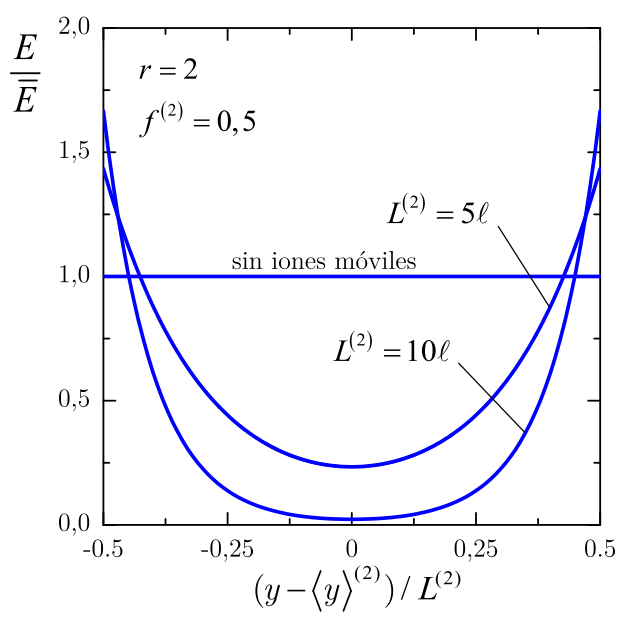

b)

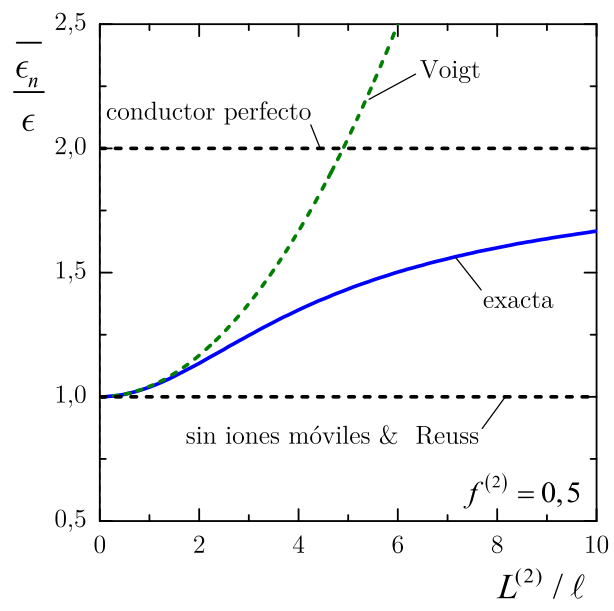

d)

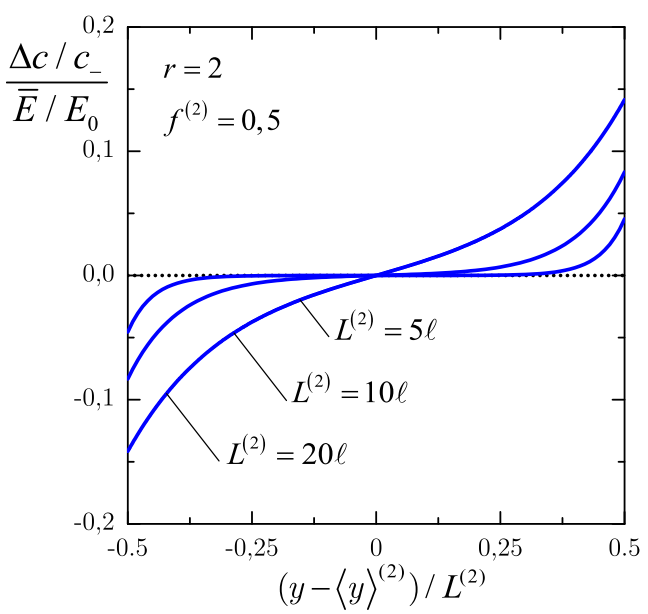

Figura 7.2: Predicciones para materiales laminados con $\epsilon^{(1)}=\epsilon^{(2)}=\epsilon, \ell^{(1)} \rightarrow \infty \mathrm{y}$ $\ell^{(2)}=\ell$ sometidos a campos eléctricos de baja intensidad: a) permitividad efectiva en función de la fracción volumétrica $f^{(2)}$, para varios espesores de la capa semiconductora; b) permitividad efectiva en función del espesor de la capa semiconductora para $f^{(2)}=0,5$; c) distribución de campo eléctrico y d) perfil de concentración de exceso de iones dentro de la fase semiconductora.

curvas para la permitividad efectiva $\bar{\epsilon}_{n}$, normalizada mediante la permitividad local $\epsilon$, en función de la fracción volumétrica y del espesor de la capa semiconductora. En ausencia de iones móviles, el medio es en realidad homogéneo y la permitividad efectiva es simplemente la permitividad local. En presencia de iones móviles, sin embargo, la permitividad efectiva varía considerablemente con la morfología microestructural y exhibe un efecto de talla muy grande. A medida que la relación $L^{(2)} / \ell$ varía desde cero a infinito, la permitividad efectiva aumenta desde el valor correspondiente a un sistema material sin cargas libres hasta un valor correspondiente a un sistema material con una fase conductora perfecta. Esta variación se debe íntegramente al efecto de polarización por cargas espaciales. 
Las figuras 7.2c y 7.2d muestran la distribución de campo eléctrico, normalizado con $\bar{E}$, y el perfil de concentración de exceso de iones $\Delta c=c_{+}-c_{-}^{(2)}$, normalizado por $c_{-}^{(2)} \bar{E} / E_{0}$, dentro de la capa semiconductora, para varios valores de la relación $L^{(2)} / \ell$. En ausencia de iones móviles, el campo eléctrico es uniforme e igual al campo macroscópico. En presencia de iones móviles, por el contrario, el campo eléctrico exhibe fuertes variaciones que crecen a medida que crece la relación $L^{(2)} / \ell$. A medida que la relación $L^{(2)} / \ell$ aumenta, el campo eléctrico disminuye en la mayor parte de la capa, pero aumenta cerca de las interfases. En el límite de $L^{(2)} / \ell \rightarrow \infty$ el campo eléctrico vale cero en toda la capa y salta a valores finitos en las interfases, como es de esperarse en una fase conductora. Estas variaciones en los campos eléctricos están acompañados por una variación en los perfiles de concentración de iones móviles y por lo tanto, de carga eléctrica. Los iones móviles son drenados de una mitad de la capa y son acumulados en la otra mitad. Para valores elevados de $L^{(2)} / \ell$, este proceso da lugar a regiones delgadas de agotamiento y acumulación de iones móviles, cuyos espesores son del orden de la longitud de Debye y cuyas magnitudes depende de las fracciones volumétricas de las fases constituyentes. La mayor parte de la caída del potencial electrostático se produce dentro de estas regiones.

Para comparar las cotas elementales con la respuesta exacta resulta conveniente escribir el límite de Voigt (6.77) como

$$
\bar{\epsilon}_{V}=f^{(1)} \epsilon_{*}^{(1)}+f^{(2)} \epsilon_{*}^{(2)} \quad \text { con } \quad S(\lambda)=1+\frac{\lambda^{2}}{12}
$$

y la cota de Reuss (6.80) como

$$
\bar{\epsilon}_{R}=\left(\frac{f^{(1)}}{\epsilon_{*}^{(1)}}+\frac{f^{(2)}}{\epsilon_{*}^{(2)}}\right)^{-1} \quad \operatorname{con} \quad S(\lambda)=1,
$$

donde las permitividades equivalentes $\epsilon_{*}^{(r)}$ están definidas nuevamente por $(7.2)_{1}$. Entonces, la cota de Reuss tiene la misma forma funcional que la permitividad efectiva exacta pero no predice un efecto de amplificación debido a polarización de cargas espaciales, mientras que la cota de Voigt tiene otra forma funcional pero predice un efecto de amplificación. En la figura 7.1b se muestra una comparación de los distintos factores de amplificación. Los factores de Voigt y Reuss acotan efectivamente por arriba y por debajo al factor exacto. Curiosamente, el factor de amplificación de Voigt es exacto al tercer orden en $\lambda$, ver expresiones $(7.2)_{2}$ y $(7.3)_{2}$. La cota de Voigt para la permitividad efectiva resulta ser entonces bastante exacta para valores bajos de $L^{(2)} / \ell$, ver figuras 7.2 a y $7.2 \mathrm{~b}$. Sin embargo, para valores mayores de $L^{(2)} / \ell$ se vuelve inútil, como era de esperar por el hecho bien conocido de que los límites de tipo Voigt no quedan acotados cuando al menos una fase es un conductor perfecto. 


\subsubsection{Respuesta no lineal}

Bajo campos eléctricos de intensidad arbitraria, la respuesta material es dictada por la densidad de energía complementaria efectiva (6.69). Esta densidad de energía se calcula aquí por medio de una discretización de elementos finitos del funcional unidimensional y la subsecuente minimización con respecto a las amplitudes nodales del potencial eléctrico. En el Apéndice A.2 pueden encontrarse detalles de estos cálculos.

En la figura 7.3 se presentan las predicciones para un laminado con $\epsilon^{(1)}=\epsilon^{(2)}=\epsilon$, $\ell^{(1)} \rightarrow \infty, \ell^{(2)}=\ell$ y $f^{(2)}=0,9$. En las figuras 7.3 a y 7.3 b se muestran curvas para el desplazamiento eléctrico macroscópico $\bar{D}$, normalizado con $D_{0}=\epsilon E_{0}$, y para la permitividad tangente $\bar{\xi}=\partial \bar{D} / \partial \bar{E}$, normalizada con $\epsilon$, en función de la intensidad de campo eléctrico, para diversos valores de la relación $L^{(2)} / \ell$. En ausencia de iones móviles, la respuesta es lineal y la permitividad tangente es simplemente $\epsilon$. En presencia de iones móviles, por otro lado, la respuesta exhibe un carácter bilineal y la permitividad tangente tiene una transición suave desde la pendiente inicial a la final. La pendiente inicial está dada por (7.1) y por lo tanto depende fuertemente del espesor de las capas semiconductoras, mientras que la pendiente final está dada por $\epsilon$ y por lo tanto es independiente del tamaño de la microestructura. La transición refleja el crecimiento y posterior saturación de la polarización de cargas espaciales a medida que aumenta la intensidad del campo eléctrico. La polarización de cargas espaciales macroscópica en estos sistemas materiales está dada simplemente por $\bar{P}_{s c}=\bar{D}-\epsilon \bar{E}$, cantidad que se muestra en la figura 7.3c. Se observa que el nivel de saturación depende en gran medida del espesor de las capas semiconductoras. Esto se corresponde con la totalidad de iones móviles que son drenados completamente de la mayor parte de la lámina y concentrados en las interfases bloqueantes, véase la figura 7.3d. Un simple cálculo en términos de ese perfil de concentración límite produce

$$
\frac{\bar{P}_{s c}^{s a t}}{D_{0}}=\frac{1}{2}\left(\frac{L^{(2)}}{\ell}\right)^{2} .
$$

Es decir, la polarización de cargas espaciales es proporcional al cuadrado del espesor de las capas semiconductoras. A medida que $\bar{E} \rightarrow \infty$, la respuesta macroscópica tiende asintóticamente a $\bar{D}=\epsilon \bar{E}+\bar{P}_{s c}^{s a t}$.

Es posible también comparar las predicciones generadas por las cotas elementales y por el método de comparación lineal con la solución exacta. La estimación de 
a)

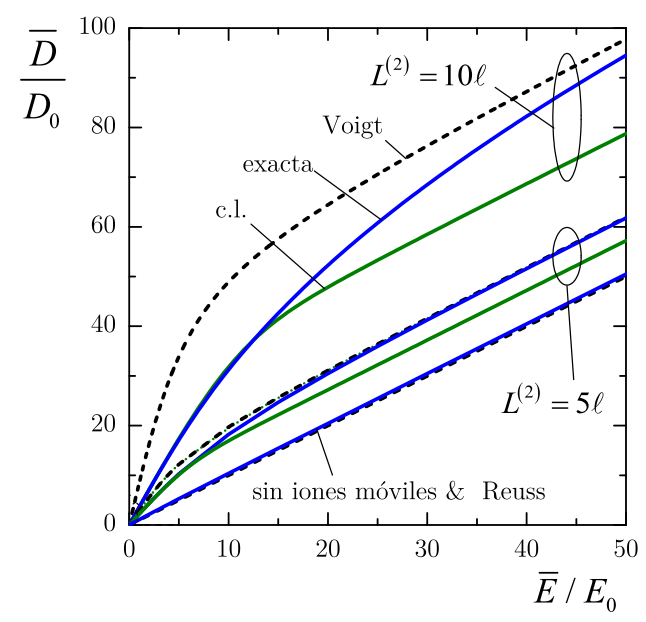

c)

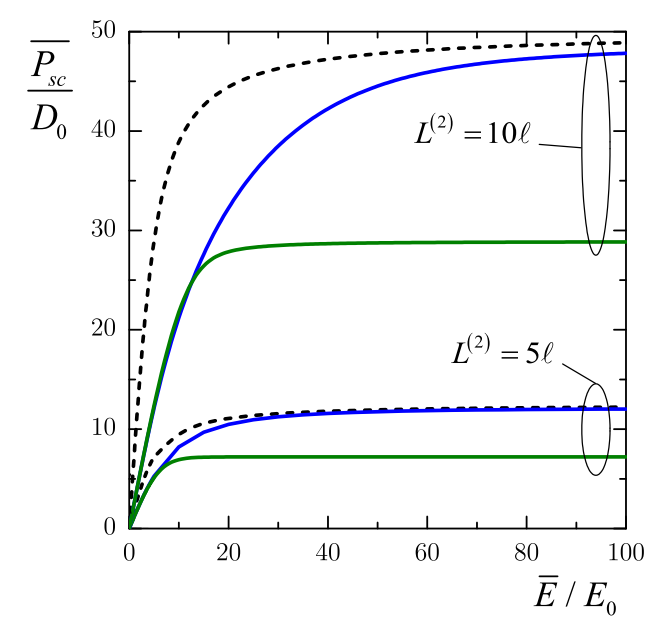

e)

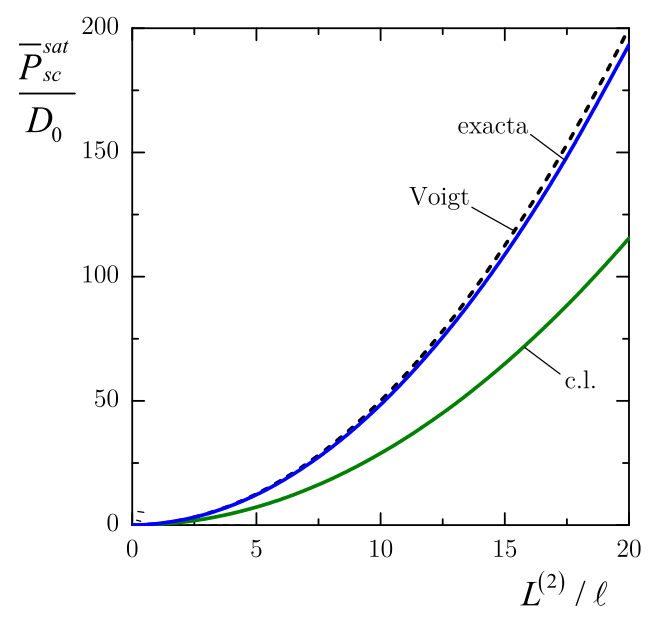

b)

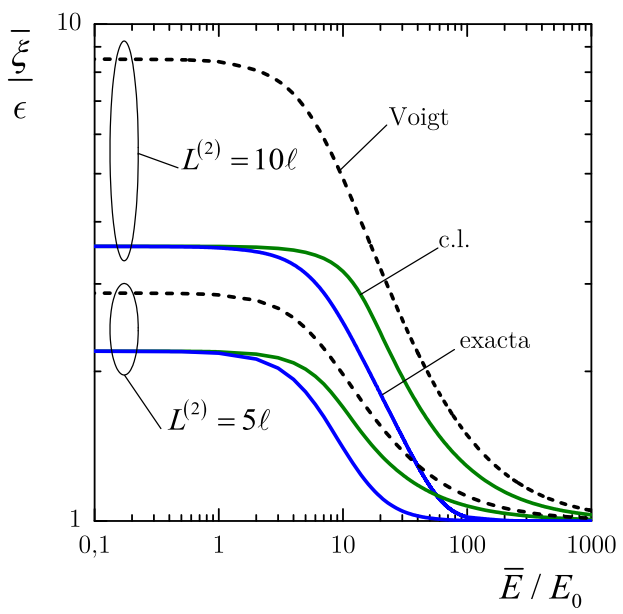

d)

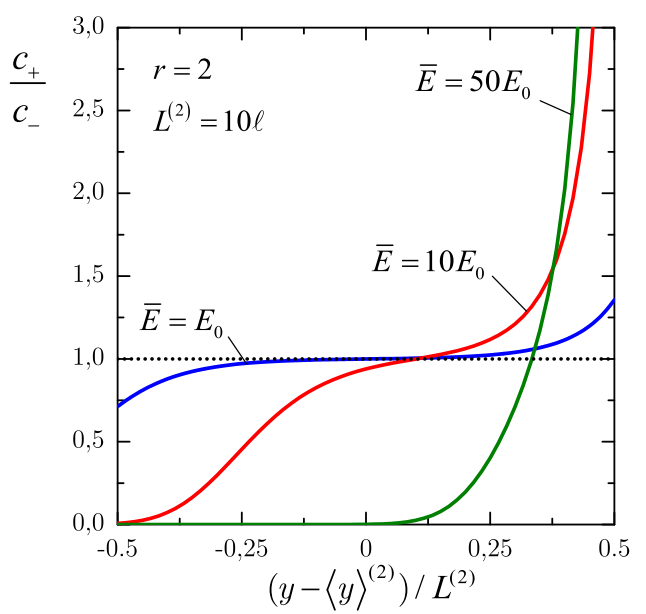

f)

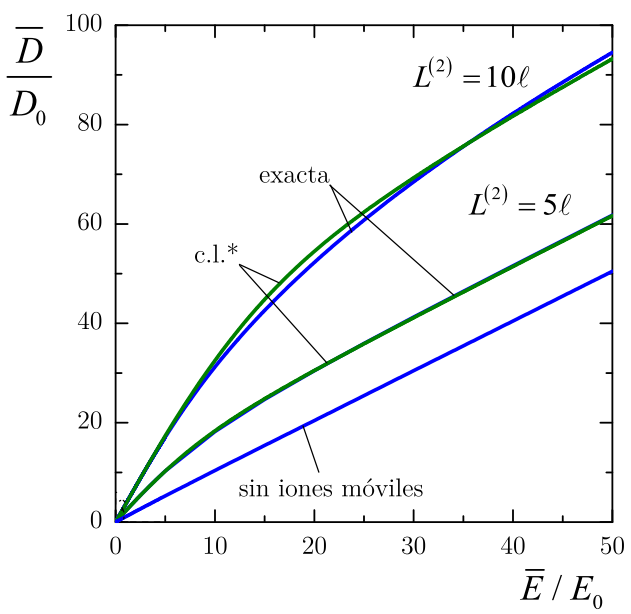

Figura 7.3: Predicciones para materiales laminados con $\epsilon^{(1)}=\epsilon^{(2)}=\epsilon, \ell^{(1)} \rightarrow \infty, \ell^{(2)}=\ell$, $f^{(2)}=0,9$, sometidos a campos eléctricos de intensidad arbitraria: a \& f) desplazamiento eléctrico macroscópico b) permitividad tangente, y c) polarización de carga espacial en función de la intensidad de campo eléctrico; d) perfil de concentración de iones móviles dentro de la capa semiconductora; e) nivel de saturación de la polarización de cargas espaciales en función del espesor de la capa semiconductora. 
comparación lineal (c.l.) para la respuesta macroscópica está dada por

$$
\bar{D}=\left(\frac{f^{(1)}}{\epsilon_{*}^{(1)}}+\frac{f^{(2)}}{\epsilon_{*}^{(2)}}\right)^{-1} \bar{E} \quad \text { con } \quad \epsilon_{*}^{(r)}=\epsilon^{(r)} S\left(L^{(r)} / \ell_{0}^{(r)}\right)
$$

donde la función $S$ está definida por $(7.2)_{2}$ y las longitudes de Debye de comparación $\ell_{0}^{(r)}$ son soluciones de las ecuaciones $(6.83)$ con las variables $m^{(r)}$ dadas por

$$
m^{(r)}=\frac{\ell_{0}^{(r)}}{2 L^{(r)}} S^{\prime}\left(L^{(r)} / \ell_{0}^{(r)}\right)\left[\frac{f^{(r)}}{\epsilon_{*}^{(r)}}\left(\frac{f^{(1)}}{\epsilon_{*}^{(1)}}+\frac{f^{(2)}}{\epsilon_{*}^{(2)}}\right)^{-1} \frac{\bar{E}}{E_{0}}\right]^{2}
$$

A su vez, la respuesta macroscópica predicha por las cotas de Reuss y Voigt están dadas por

$$
\begin{aligned}
\bar{D}= & \left(\frac{f^{(1)}}{\epsilon^{(1)}}+\frac{f^{(2)}}{\epsilon^{(2)}}\right)^{-1} \bar{E} \text { y } \\
\bar{D}= & \left(f^{(1)} \epsilon^{(1)}+f^{(2)} \epsilon^{(2)}\right) \bar{E}+ \\
& +\sum_{r=1}^{2} F z c_{-}^{(r)} L^{(r)}\left[\frac{f^{(r)}}{2} \frac{\bar{E}}{E_{0}} \operatorname{coth}\left(\frac{f^{(r)}}{2} \frac{\bar{E}}{E_{0}}\right)-1\right]\left(\frac{\bar{E}}{E_{0}}\right)^{-1},
\end{aligned}
$$

respectivamente. Una comparación para materiales laminados con $\epsilon^{(1)}=\epsilon^{(2)}=\epsilon$, $\ell^{(1)} \rightarrow \infty, \ell^{(2)}=\ell$ y $f^{(2)}=0,9$ se muestran en la figura 7.3. Para intensidades de campo bajas se observa en la figura 7.3a que las predicciones de Voigt y Reuss respectivamente sobrestiman y subestiman el desplazamiento eléctrico macroscópico mientras que las predicciones de c.l. son más precisas, como era de esperar de sus derivaciones. Para intensidades de campo elevadas, sin embargo, las predicciones de c.l. capturan la pendiente correcta, pero subestiman considerablemente el desplazamiento eléctrico, al punto de volverse menos precisas que las predicciones de Voigt. Las comparaciones presentadas en la figura 7.3c muestran que el método de c.l. está en realidad subestimando la polarización de cargas espaciales. En efecto, el análisis asintótico de las expresiones (7.6)-(7.9) muestra que la polarización de cargas espaciales saturada predicha por la cota de Voigt coincide exactamente con (7.5), mientras que las estimaciones de c.l. predicen

$$
\frac{\bar{P}_{s c}^{s a t}}{D_{0}}=\frac{\sqrt{3}}{6}\left(\frac{L^{(2)}}{\ell}\right)^{2}
$$

que queda un $40 \%$ por debajo, véase la figura 7.3e. Entonces, el método de comparación lineal de la sección 6.4.3 aparenta tener dificultades para capturar adecuadamente las grandes variaciones espaciales del campo electrostático que se desarrollan en la cercanía de las interfases para intensidades de campo elevadas. Una posible solución 
es utilizar un medio lineal de comparación refinado en el cual las propiedades de comparación $\alpha_{0}^{(r)}$ y $\beta_{0}^{(r)}$ puedan variar dentro de cada microdominio $r$, especialmente en el entorno de las interfases. En este caso, debe observarse que al permitir que las propiedades de comparación varíen punto a punto, la estimación recupera la respuesta exacta. De cualquier manera, cualquier ganancia en la precisión será a expensas de la complejización de la formulación subyacentes. Alternativamente, un enfoque más práctico consiste en introducir modificaciones ad-hoc en las expresiones (7.6)-(7.7) de tal manera que las predicciones de c.l. recuperen la saturación exacta de la polarización de cargas espaciales. El análisis asintótico de estas expresiones cuando $\bar{E} \rightarrow \infty$ revela que una de las modificaciones posibles consiste en multiplicar el lado derecho de la expresión (7.7) por un factor de la forma

$$
M=\frac{\sqrt{3}}{3}+\left(\frac{\sqrt{3}}{3}-1\right) \tanh \left(\frac{L^{(2)}}{\ell}\left(\frac{\bar{E}}{E_{0}}\right)^{-1}\right) .
$$

Las predicciones resultantes (c.l. ${ }^{*}$ ) se comparan con los resultados exactos en la figura 7.3f. Se observa que esta sencilla modificación produce en efecto resultados precisos para todo el rango de parámetros materiales considerados sin complejizar significativamente la formulación subyacente.

\subsection{Materiales particulados}

\subsubsection{Partículas en suspensión diluida}

Se analiza ahora el caso correspondiente a un sólido dieléctrico cuya microestructura consiste de una fase de matriz continua $(r=1)$ que contiene una suspensión diluida de partículas esféricas $(r=2)$. Se considera que todas las partículas son de radio a y están bien separadas. Ambas fases se caracterizan mediante densidades de energía complementarias de la forma (6.19), pero solamente se considera que existen iones móviles en las partículas. En la figura 7.4a se muestra la celda unitaria considerada.

\section{Respuesta lineal}

Para intensidades de campo eléctrico bajas, la densidad de energía complementaria efectiva puede ser caracterizada por el tensor de permitividad efectiva $\overline{\boldsymbol{\epsilon}}$, definido por (6.72). Además, en vista de la isotropía global, $\overline{\boldsymbol{\epsilon}}=\bar{\epsilon} \mathbf{I}$. Haciendo uso de las 
ecuaciones de Euler-Lagrange, la expresión (6.72) puede reescribirse como

$$
\begin{array}{r}
\frac{1}{2} \bar{\epsilon} \overline{\mathbf{E}}^{2}=\frac{1}{2} \epsilon^{(1)} \overline{\mathbf{E}}^{2}+\frac{f^{(2)}}{2}\left[\left(\epsilon^{(2)}-\epsilon^{(1)}\right)\langle-\nabla \phi\rangle^{(2)}+\right. \\
\left.+\frac{F^{2} z^{2} c_{-}^{(2)}}{R T}\left\langle\left(\langle\phi\rangle^{(2)}-\phi\right) \mathbf{y}\right\rangle^{(2)}\right] \cdot \overline{\mathbf{E}}
\end{array}
$$

donde $\phi$ es el potencial electrostático solución de la minimización. Entonces, la energía efectiva exacta puede determinarse únicamente conociendo el campo electrostático dentro de las partículas. Este campo es encontrado en el Apéndice B resolviendo el problema de una partícula esférica en un medio infinito. Introduciendo el campo resultante en (7.12) y llevando a cabo los promedios volumétricos se obtiene

$$
\frac{\bar{\epsilon}}{\epsilon^{(1)}}=1+f^{(2)} \beta \quad \text { con } \quad \beta=3 \frac{\epsilon_{*}^{(2)}-\epsilon^{(1)}}{\epsilon_{*}^{(2)}+2 \epsilon^{(1)}} .
$$

En esta última expresión, el factor $\beta$ caracteriza la contribución de la fase de la inclusión en la polarización total, y depende de la permitividad $\epsilon_{*}^{(2)}$ dada por

$$
\epsilon_{*}^{(2)}=\epsilon^{(2)} S(a / \ell) \quad \operatorname{con} \quad S(\lambda)=\frac{\lambda^{2}}{\lambda \operatorname{coth} \lambda-1}-2,
$$

donde $\ell$ es la longitud de Debye de la inclusión. Así, una vez más, la influencia de la polarización de cargas espaciales ingresa en las predicciones a través de una función $S$ dependiente de la talla que juega el rol de factor de amplificación de la permitividad local. Esta función se muestra en la figura 7.4c, donde se observa que es cualitativamente similar a la correspondiente para materiales laminados. Por ejemplo, la permitividad equivalente de una partícula con iones móviles cuyo radio es de dos veces y media la longitud de Debye es aproximadamente el doble que la de una partícula sin iones. El mismo factor de amplificación se puede identificar en la expresión de O’Brien (1996) o Trukhan (1963) para la permitividad efectiva de una suspensión diluida de partículas semiconductoras.

Para fines comparativos, las cotas elementales del tipo Voigt y Reuss para la permitividad efectiva también se escriben en la forma $(7.13)_{1}$, pero con

$$
\beta=\frac{\epsilon_{*}^{(2)}}{\epsilon^{(1)}}-1 \quad \mathrm{y} \quad S(\lambda)=1+\frac{\lambda^{2}}{5}
$$

para el caso de la cota de Voigt, y con

$$
\beta=\frac{\epsilon_{*}^{(2)}-\epsilon^{(1)}}{\epsilon_{*}^{(2)}} \quad \mathrm{y} \quad S(\lambda)=1
$$


a)

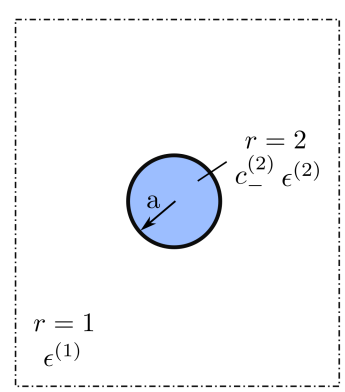

b)

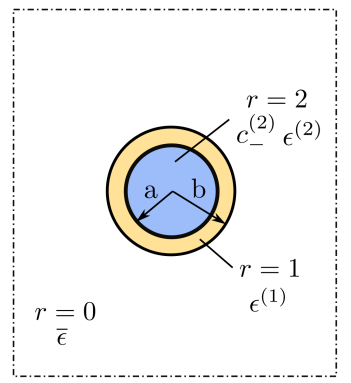

c)

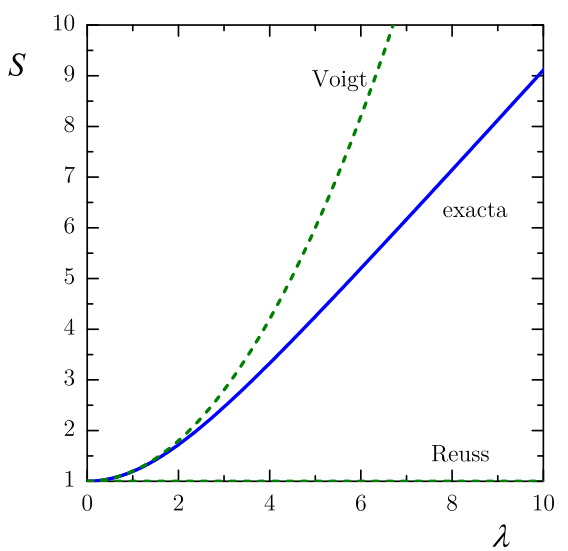

Figura 7.4: Materiales particulados: a) celda unitaria, caso diluido; b) celda unitaria, concentración arbitraria; c) factor de amplificación.

para el caso de la cota de Reuss. Estos factores de amplificación se grafican en la figura 7.1b junto con la solución exacta. Nuevamente, la cota de Reuss no predice un efecto de amplificación debido al efecto de cargas espaciales, mientras que la cota de Voigt predice un efecto de amplificación mas pronunciado que la solución exacta, más aun al aumentar $\lambda$. Curiosamente, la función $\beta$ para la cota de Voigt nuevamente coincide con la solución exacta al tercer orden de $\lambda$ —ver las expresiones $(7.14)_{2}$ y $(7.15)_{2}$.

En la figura 7.5 se presentan predicciones para un material particulado con $\epsilon^{(1)}=$ $\epsilon^{(2)}=\epsilon, \ell^{(1)} \rightarrow \infty \mathrm{y} \ell^{(2)}=\ell$. Esto significa que tanto la matriz como la partícula tienen la misma permitividad pero solamente la fase de las partículas $(r=2)$ contiene iones móviles. Las figuras 7.5a y b muestran, respectivamente, el factor $\beta$ que caracteriza la contribución de la inclusión a la polarización total y la permitividad efectiva $\bar{\epsilon}$, normalizada mediante la permitividad local $\epsilon$, en función del espesor de la capa semiconductora. En ausencia de iones móviles, el medio es en realidad homogéneo y la permitividad efectiva es simplemente la permitividad local. En presencia de iones móviles, sin embargo, la permitividad efectiva varía considerablemente con la morfología microestructural y exhibe un efecto de talla muy grande. A medida que la relación $a / \ell$ varía desde cero a infinito, la permitividad efectiva aumenta desde el valor correspondiente a un sistema material sin cargas libres hasta un valor correspondiente a un sistema material con una inclusión conductora perfecta. Esta variación se debe íntegramente al efecto de polarización por cargas espaciales. Efectivamente, en la figura 7.5a se observa que el factor de contribución de la inclusión tiende al valor correspondiente a un conductor perfecto.

En las figuras 7.5c y d se muestran la distribución de campo eléctrico y el perfil de concentración de iones móviles a lo largo del diámetro central de las partículas 
a)

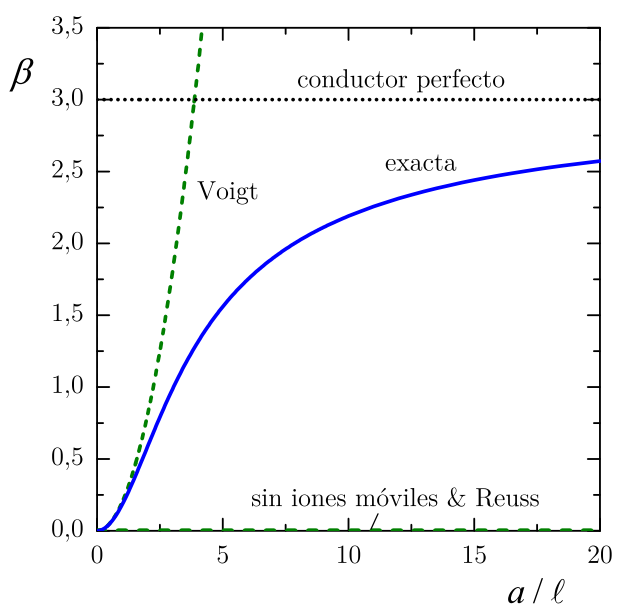

c)

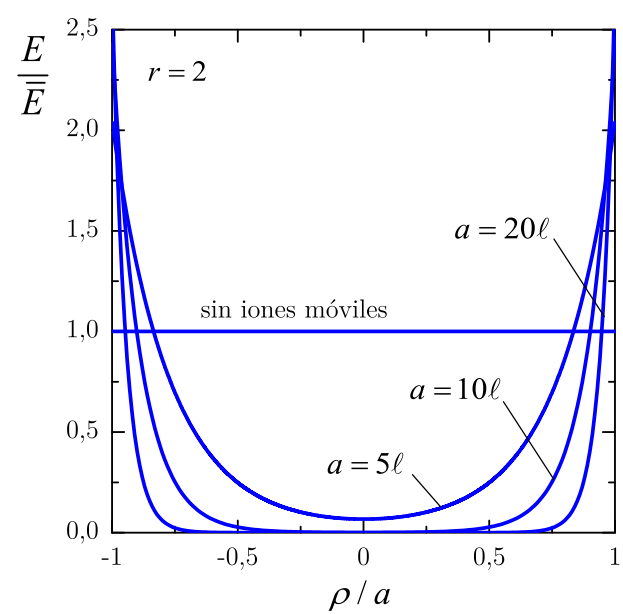

b)

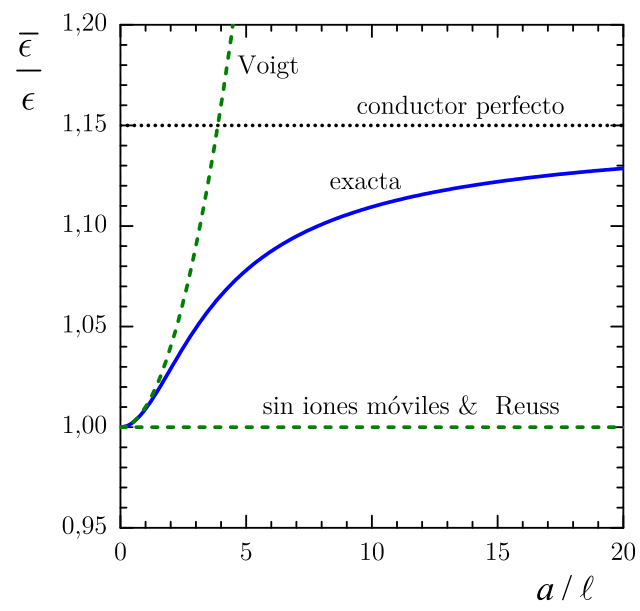

d)

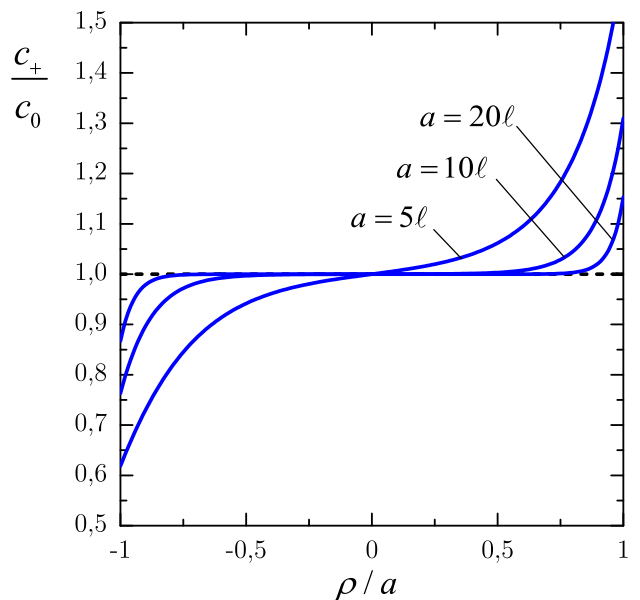

Figura 7.5: Predicciones para materiales particulados $\operatorname{con} \epsilon^{(1)}=\epsilon^{(2)}=\epsilon \mathrm{y} \ell^{(2)}=\ell$, $f^{(2)}=0,05$ : a) factor $\beta$ en función de $a / \ell$; b) permitividad efectiva en función de $a / \ell ; \mathrm{c}$ ) distribución de campo eléctrico y d) perfil de concentración de iones móviles a lo largo del diámetro central de las partículas en la dirección del campo aplicado, normalizado por $\bar{E}$ y por $c_{0}=c_{-}^{(2)} \bar{E} / E_{0}$, con $E_{0}=R T / F z a$.

en la dirección del campo aplicado, normalizadas respectivamente por $\bar{E}$ y por $c_{0}=c_{-}^{(2)} \bar{E} / E_{0}$, con $E_{0}=R T / F z a$, para varios valores de $a / \ell$. Las variaciones espaciales resultan ser cualitativamente similares a las ya discutidas en el contexto de materiales laminados. Una vez más, se observa que la presencia de iones móviles produce fuertes variaciones espaciales del campo eléctrico, en marcado contraste con el campo uniforme predicho por el resultado clásico para partículas no conductoras. 


\subsubsection{Material con partículas con concentraciones arbitrarias}

\section{Respuesta lineal}

Las predicciones presentadas anteriormente solamente son válidas para concentraciones bajas de partículas bien espaciadas. Las predicciones para sistemas materiales con altas concentraciones de partículas deben tener en cuenta las interacciones entre partículas. En ausencia de iones móviles, una estrategia popular para modelar esas interacciones consiste en recurrir a una clase especial de microestructuras conocidas como conjuntos de esferas compuestas (véase, por ejemplo, Milton, 2002). Ahora bien, la clave para la construcción de estas microestructuras es el hecho de que la contribución de cada esfera compuesta a la respuesta efectiva del conjunto es la misma, independientemente del tamaño absoluto de la esfera. Por lo tanto, este proceso de construcción falla en presencia de iones móviles a menos que se asuma una longitud de Debye diferente para cada esfera de tal manera que la relación $a / \ell$ sea fija para todas las esferas compuestas. Un enfoque equivalente fue propuesto por Hashin (2001) en el contexto de conductores compuestos con interfaces imperfectas, donde los efectos de talla también están presentes. En el presente contexto, esto implica suponer que la concentración molar de contraiones fijos dentro de una partícula es inversamente proporcional al cuadrado del radio de la partícula. A pesar de esto, es posible que esto represente una buena aproximación para sistemas polidispersos con una concentración molar de iones única y fracciones volumétricas moderadas, ya que la solución tiende al caso diluido. Cabe señalar además que Boersma y van Turnhout (1998) utilizaron una estrategia similar para derivar estimaciones de tipo autoconsistente.

Siguiendo esta estrategia se analiza ahora el caso correspondiente a un sólido que contiene una partícula esférica $(r=2)$ recubierta por una capa de otro material dieléctrico $(r=1)$ y que se encuentran embebidas en una matriz infinita $(r=0)$. Se considera que la partícula es de radio $a$ y el recubrimiento de radio $b$. Todas las fases se caracterizan mediante densidades de energía complementarias de la forma (6.19), pero solamente se considera que existen iones móviles en la partícula. En la figura $7.4 \mathrm{~b}$ se muestra la celda unitaria considerada. La clave de este desarrollo consiste en suponer que existe un valor de permitividad $\bar{\epsilon}$ para la matriz infinita que no se ve alterado al agregar o quitar la partícula recubierta. Debido al carácter local de las ecuaciones del problema, es posible agregar otras esferas recubiertas de distintos tamaños hasta llenar completamente el espacio, con lo que se obtendría un material bifásico de fases $r=1$ y $r=2$. Como ya fue mencionado, dado que cada esfera que se agrega debe ser una versión en escala de la anterior, surge entonces la necesidad de asumir que la relación $a / \ell$ sea fija para todas las esferas. 
a)

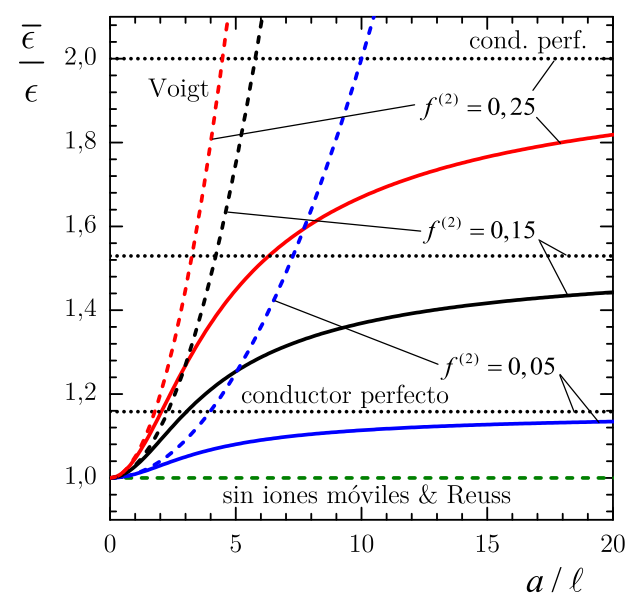

b)

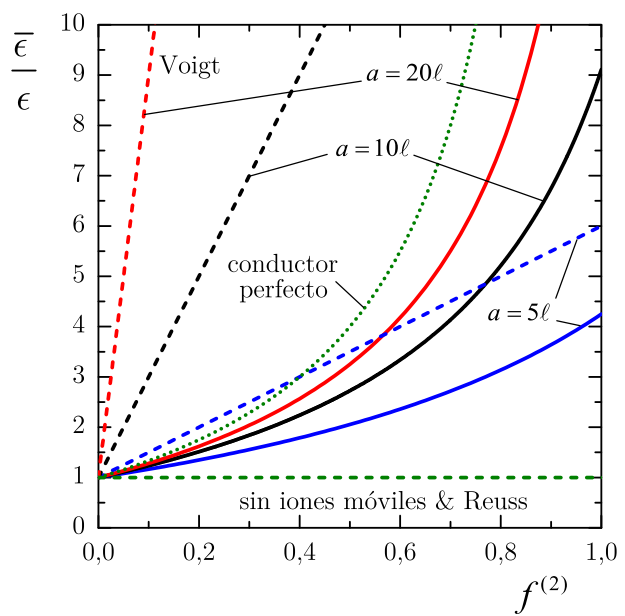

Figura 7.6: Predicciones para materiales particulados con $\epsilon^{(1)}=\epsilon^{(2)}=\epsilon$ y $\ell^{(2)}=\ell$ y concentración arbitraria: a) permitividad efectiva en función de $a / \ell$; b) permitividad efectiva en función de la concentración de partículas $f^{(2)}$.

La energía efectiva exacta del sistema puede determinarse únicamente conociendo el campo electrostático dentro de la partícula y su recubrimiento. Esta campo es encontrado en el Apéndice B.2 resolviendo el problema de una partícula esférica con recubrimiento en un medio infinito con un campo eléctrico uniforme. Introduciendo el campo resultante en (6.72) y llevando a cabo los promedios volumétricos se obtiene

$$
\frac{\bar{\epsilon}}{\epsilon^{(1)}}=1+\frac{3 f^{(2)}\left(\epsilon_{*}^{(2)}-\epsilon^{(1)}\right)}{3 \epsilon^{(1)}+\left(1-f^{(2)}\right)\left(\epsilon_{*}^{(2)}-\epsilon^{(1)}\right)},
$$

donde la permitividad $\epsilon_{*}^{(2)}$ está nuevamente dada por la expresión (7.14).

Así, una vez más, la influencia de la polarización de cargas espaciales ingresa en las predicciones a través de una función dependiente de la talla $S$ que juega el rol de factor de amplificación de la permitividad local y que coincide con el obtenido para la partícula en suspensión diluida. A su vez, la expresión (7.17) tiene la misma forma que la expresión obtenida por Milton (2002) para un conjunto de esferas recubiertas en el contexto de conductividad eléctrica, con la diferencia que la permitividad para la inclusión aquí se encuentra afectada por el factor $S$ dependiente de las presencia de iones en la partícula.

En la figura 7.6 se presentan predicciones para un material particulado con $\epsilon^{(1)}=$ $\epsilon^{(2)}=\epsilon, \ell^{(1)} \rightarrow \infty$ y $\ell^{(2)}=\ell$. Esto significa que tanto la matriz como la partícula tienen la misma permitividad pero solamente la fase de las partículas $(r=2)$ contiene iones móviles. Las figuras 7.6a y b muestran, respectivamente, la permitividad efectiva $\bar{\epsilon}$, normalizada mediante la permitividad local $\epsilon$, en función del espesor de la capa 
semiconductora y de la concentración de partículas. Una vez más, en ausencia de iones móviles, el medio es en realidad homogéneo y la permitividad efectiva es simplemente la permitividad local. En presencia de iones móviles, sin embargo, la permitividad efectiva varía considerablemente con la morfología microestructural y exhibe un efecto de talla muy grande. A medida que la relación a/ $\ell$ varía desde cero a infinito, la permitividad efectiva aumenta desde el valor correspondiente a un sistema material sin cargas libres hasta un valor correspondiente a un sistema material con una inclusión conductora perfecta, tal como se observó para el caso diluido. Efectivamente, para valores de $f^{(2)}$ pequeños, la expresión (7.17) tiende a la expresión diluida (7.13).

Teniendo en cuenta los resultados obtenidos en la sección 7.2.2 con el método de comparación lineal aplicado a materiales laminados, no se utiliza aquí este método para obtener estimaciones para la respuesta no lineal del material particulado. En efecto, se observó que las estimaciones mediante el método de comparación lineal pueden ser menos precisas que la cota de Voigt, por lo que se omiten esos resultados para medios particulados. 


\section{Conclusiones y trabajo futuro}

Se ha derivado un modelo multiescala para la respuesta electromecánica de compuestos ferroeléctricos bifásicos con microestructuras e historias de carga complejas. Este modelo es capaz de reproducir características esenciales de la respuesta de materiales ferróicos, como ser curvas de tensión-deformación no lineales, curvas de ciclos de histéresis, y rotación de dipolos. El comportamiento electrodeformable de las fases constitutivas se describe mediante su densidad de energía libre de Helmholtz y un potencial de disipación convexos propuestos por Miehe y Rosato (2011) en conformidad con el formalismo de materiales estándar generalizados. Esto garantiza la consistencia termodinámica de las predicciones resultantes.

En primer lugar se realizó una evaluación del modelo a nivel local, sin considerar la presencia de segundas fases. Se comprobó que el modelo es capaz de reproducir las principales características de los materiales ferroeléctricos y ferromagnéticos como ser los ciclos de histéresis y el comportamiento frente a campos no alineados con la polarización remanente presente en el espécimen. Sin embargo, se encontró que frente a estados de carga con presencia de tensiones mecánicas se pueden obtener respuestas que no se condicen con la física del problema y que son atribuibles a la falta de convexidad en la densidad de energía libre con respecto a la polarización irreversible. Esta característica inesperada exige que el modelo sea utilizado con precaución, incluso para niveles bajos de tensión para los cuales no deberían presentarse inconvenientes, de acuerdo a lo que afirman algunos de sus impulsores (por ejemplo, McMeeking y Landis, 2002).

Por otro lado, las capacidades de la teoría fueron evaluadas en el contexto de microgeometrías resolubles, considerando un modelo de laminados secuenciales para compuestos particulados. En particular, se analizaron diversos casos de estudio para diferentes sistemas materiales representativos consistentes de una fase ferroeléctrica con inclusiones de partículas metálicas, ferromagnéticas y poros.

Inicialmente se analizaron especímenes sin restricciones mecánicas sometidos únicamente a cargas eléctricas para los cuales se pudo concluir que la presencia de partículas metálicas reducen el acoplamiento electromecánico y los coeficientes piezoeléctricos analizados, mientras que la porosidad no afecta la electrodeformación, pero produce gran variación de los coeficientes piezoeléctricos. Durante el análisis de los coeficientes piezoeléctricos, también se utilizó la popular aproximación que considera que la polarización irreversible es uniforme y constante en todo el sólido. Comparando esos resultados con los obtenidos con el modelo desarrollado en este trabajo se pudo 
concluir que los métodos de predicción asumiendo una distribución uniforme de la polarización pueden subestimar la influencia de las inclusiones en algunos coeficientes piezoeléctricos al no contemplar las fluctuaciones en la polarización remanente del material.

Para el caso de los especímenes con inclusiones ferromagnéticas se observó que el problema de no convexidad detectado en el modelo genera resultados que no son físicamente admisibles, por lo que el estudio de sistemas multiferróicos con metodologías como la presentada debe realizarse con cautela y prestando gran atención a la combinación de parámetros de las fases utilizadas para no desencadenar la problemática detectada.

También fueron estudiados sistemas materiales sometidos a cargas electromecánicas y, en la misma línea, se pudo concluir que para niveles de tensión por debajo del límite material de las fases se presentan comportamientos que no resultan ser físicamente admisibles, requiriendo hacer un análisis exhaustivo para asegurar la validez de las predicciones para condiciones de carga arbitrarias.

El análisis presentado indica entonces que la clase de teorías fenomenológicas utilizada como base para el modelo de laminados secuenciales solamente pueden generar predicciones confiables para historias de carga complejas si las tensiones mecánicas permanecen por debajo de cierto límite, que puede ser del orden de algunos megapascales en el caso de cerámicos ferroeléctricos típicos, y muy por debajo de un megapascal en el caso aleaciones ferromagnéticas típicas. Bajo niveles de tensión más elevados, las predicciones se vuelven numéricamente inestables o la solución no es única, dependiendo de la magnitud relativa de los diversos parámetros materiales. Esto es una consecuencia directa de la dependencia no convexa en la densidad de dipolos permanentes asumida para la energía elástica.

A pesar de que no se han considerado otras dependencias funcionales en este análisis, la no convexidad parece inevitable si el conjunto de variables internas que describen la distribución de dipolos permanentes se debe restringir a una única variable vectorial, como sucede en esta clase de teorías. Esto es así debido a que la física de la ferroelectricidad y ferromagnetismo requiere que el tensor deformación asociado a los dipolos permanentes sea una función impar de esa variable vectorial, mientras que la energía libre debe depender cuadráticamente de ese tensor deformación. Cabe destacar también que es esperable que este tipo de problemas persista en versiones más sofisticadas de estas teorías que hacen uso de un tensor de deformación irreversible como una variable interna adicional de modo de poder capturar efectos ferroelásticos, como los propuestos por Kamlah (2001) y Landis (2002), dado que los niveles de tensión que típicamente se requieren para producir ferroelasticidad son 
significativamente mayores que aquellos indicados previamente. Puede mencionarse además que se ha demostrado recientemente que estos tipos de modelos presentan problemas adicionales que surgen de la forma asumida para el potencial de disipación (Stark et al., 2016a).

En este contexto puede concluirse entonces que las teorías fenomenológicas para ferroelectricidad y ferromagnetismo requieren del uso de variables internas enriquecidas que describan la distribución de dipolos permanentes dentro del sólido. Esfuerzos en esas líneas ha sido realizados por Kamlah (2001), Mehling et al. (2007) y, más recientemente, Stark et al. (2016b), pero las teorías resultantes aún no han sido evaluadas exhaustivamente.

Alternativamente, puede considerarse el modelado micromecánico teniendo en cuenta el carácter policristalino de los ferrocerámicos. Si bien la mayor parte de los modelos desarrollados hasta el momento presentan el inconveniente de ser complejos y tener un alto costo computacional para su aplicación a problemas de ingeniería típicos, trabajos recientes de Tan y Kochmann (2017) se presentan como una alternativa a tener en cuenta. El modelo constitutivo propuesto para cerámicos ferroeléctricos policristalinos relaciona los mecanismos de conmutación de dominios y las transiciones de fases en la microescala con la respuesta material a escala macroscópica. El marco propuesto es simple y por lo tanto eficiente, manteniendo sin embargo características y mecanismos microestructurales claves al introducir una mezcla de dominios en lugar de utilizar una descripción fenomenológica para la polarización remanente. Si bien el modelo no aporta detalles microestructurales locales ni tiene en cuenta la interacción entre bordes de granos, es lo suficientemente eficiente para permitir realizar cálculos de elementos finitos de gran escala. Es en esta línea de trabajo donde se espera que surjan mejoras en la modelización de dispositivos ferroeléctricos en el futuro cercano.

Con respecto al análisis de materiales dieléctricos con presencia de iones móviles, se desarrolló el marco teórico para el problema considerando la existencia de cargas libres. El comportamiento del material se describe nuevamente mediante una densidad de energía libre de Helmholtz que caracteriza completamente su respuesta y cuya convexidad está en conformidad con el formalismo de materiales estándar generalizados. Esto garantiza la consistencia termodinámica de las predicciones resultantes.

Se formuló el problema multiescala, en donde queda en evidencia la dependencia de la polarización macroscópica respecto a la polarización de cargas espaciales generadas por los cationes desplazados y los contraiones fijos. Se presentaron cotas y estimaciones para la respuesta de este tipo de sistemas que permiten obtener de manera simple la respuesta sin tener que resolver la complejidad matemática, la 
variación espacial y la no linealidad de la respuesta local.

A fines ilustrativos se presentó la respuesta para sistemas materiales modelo, donde se evidenció la importancia del efecto de las cargas libres en las fases semiconductoras y su gran influencia en la respuesta final del material. Sin embargo, la generación de predicciones realistas para sistemas materiales complejos tales como cerámicos policristalinos, polímeros rellenados con nanopartículas y, en particular, la aplicación a cerámicos ferroeléctricos debe contemplar el hecho que la polarización y las cargas eléctricas interactúan a través del potencial electrostático en el material de manera no trivial. Esto requerirá, en general, descripciones microestructurales y constitutivas más elaboradas junto con algoritmos numéricos adecuados para resolver las ecuaciones de campo microscópicas consiguientes. En relación a esto, cabe señalar que las ecuaciones de campo microscópicas presentadas en (6.29)-(6.31) pueden reescribirse en una ecuación algebraica-integral no lineal del tipo de Lippmann-Schwinger para los potenciales electrostáticos y químicos, y por lo tanto puede ser resuelto numéricamente por medio de esquemas iterativos basados en la transformada rápida de Fourier, similares a los ya empleados en el contexto de elastoplasticidad (por ejemplo, Michel et al., 2001; Moulinec y Suquet, 1998). Estos esquemas numéricos han demostrado ser robustos incluso cuando los campos locales son propensos a localizar (por ejemplo, Idiart et al., 2006, 2009), como es el caso del campo de potencial electrostático en el presente contexto. Por otra parte, la generación de predicciones realistas para condiciones de carga dinámicas requerirá tener en cuenta los efectos disipativos (por ejemplo, Suryanarayana y Bhattacharya, 2012; Xiao y Bhattacharya, 2008) y, por lo tanto, una mayor extensión de la formulación. Una estrategia posible consiste en utilizar el marco de materiales estándar generalizados junto con los principios variacionales incrementales apropiados en línea con trabajos recientes sobre elastoplasticidad y ferroelectricidad (por ejemplo, Idiart, 2014; Idiart y Lahellec, 2016; Lahellec y Suquet, 2013).

Se espera que estos desarrollos teóricos generen, finalmente, herramientas de análisis confiables que nos permitan diseñar dispositivos electrodeformables complejos para sistemas y equipos en diversas ramas de la ingeniería. 


\section{Referencias}

Arms S.W., Townsend C.P., Churchill D.L., Galbreath J.H., Mundell S.W. y Lane H. (2005): Power Management for Energy Harvesting Wireless Sensors. En SPIE International Symposium on Smart Structures \& Smart Materials, Marzo, 1-9.

Asare T.A., Poquette B.D., Schultz J.P. y Kampe S.L. (2012): Investigating the vibration damping behavior of barium titanate $\left(\mathrm{BaTiO}_{3}\right)$ ceramics for use as a high damping reinforcement in metal matrix composites. Journal of Materials Science, 47(6), 2573-2582.

Auld B.A. (1969): Application of microwave concepts to the theory of acoustic fields and waves in solids. IEEE Transactions on Microwave Theory and Techniques, MTT-17(11), 800-811.

Avellaneda M. y Harshe G. (1994): Magnetoelectric Effect in Piezoelectric/Magnetostrictive Multilayer (2-2) Composites. Journal of Intelligent Material Systems and Structures, 5(4), 501-513.

Barolin S.A., De La Rubia M.A., Terny S., Rubio-Marcos F., De Sanctis O., Alonso R.E., Fernández Lozano J.F. y De Frutos J. (2014): Respuesta Ferro-Piezoeléctrica de (K,Na,Li)(Nb,Ta,Sb) $\mathrm{O}_{3}$ Poroso. Boletín de la Sociedad Española de Cerámica y Vidrio, 53(1), 48-52.

Bassiouny E., Ghaleb A.F. y Maugin G.A. (1988): Thermodynamical formulation for coupled electromechanical hysteresis effects-I. Basic equations. International Journal of Engineering Science, 26(12), 1279-1295.

Belov A.Y. y Kreher W.S. (2005): Viscoplastic models for ferroelectric ceramics. Journal of the European Ceramic Society, 25(12 SPEC. ISS.), 2567-2571.

Blanco P.J., Sánchez P.J., de Souza Neto E.A. y Feijóo R.A. (2016): Variational Foundations and Generalized Unified Theory of RVE-Based Multiscale Models. Archives of Computational Methods in Engineering, 23(2), 191-253.

Boersma A. y van Turnhout J. (1998): Dielectric study on size effects in polymer laminates and blends. J. Polym. Sci., Part B: Polym. Phys., 36(15), 2835-2848.

Brizé V., Gruener G., Wolfman J., Fatyeyeva K., Tabellout M., Gervais M. y Gervais F. (2006): Grain size effects on the dielectric constant of $\mathrm{CaCu}_{3} \mathrm{Ti}_{4} \mathrm{O}_{12}$, ceramics. Materials Science and Engineering: B, 129(1-3), 135-138.

Bruggeman D.A.G. (1935): Berechnung verschiedener physikalischer Konstanten von heterogenen Substanzen. I. Dielektrizitätskonstanten und Leitfähigkeiten der Mischkörper aus isotropen Substanzen. Annalen der Physik, 416(7), 636-664.

Capsal J.F., David C., Dantras E. y Lacabanne C. (2012): Piezoelectric sensing 
coating for real time impact detection and location on aircraft structures. Smart Materials and Structures, 21(5), 55021.

Chen P.J. (1980): Three dimensional dynamic electromechanical constitutive relations for ferroelectric materials. International Journal of Solids and Structures, 16(12), $1059-1067$.

Chen W. y Lynch C. (1998): A micro-electro-mechanical model for polarization switching of ferroelectric materials. Acta Materialia, 46(15), 5303-5311.

Cheng H. y Torquato S. (1997): Electric-field fluctuations in random dielectric composites. Physical Review B, 56(13), 8060-8068.

Clark A. y Savage H. (1983): Magnetostriction of rare earth-Fe $\mathrm{F}_{2}$ compounds under compressive stress. Journal of Magnetism and Magnetic Materials, 31-34, 849-851.

Cule D. y Torquato S. (1998): Electric-field distribution in composite media. Phys. Rev. B, 58(18), 829-832.

Damjanovič D. (2006): Hysteresis in piezoelectric and ferroelectric materials. En G. Bertotti y I.D. Mayergoyz, eds., The Science of Hystersis, vol. 3, cap. 4, 337-465. Academic, Amsterdam; Boston.

Danas K., Idiart M.I. y Ponte Castañeda P. (2008): A homogenization-based constitutive model for two-dimensional viscoplastic porous media. Comptes Rendus Mécanique, 336(1-2), 79-90.

de Araujo C.A.P., Cuchiaro J., McMillan L., Scott M. y Scott J. (1995): Fatigue-free ferroelectric capacitors with platinum electrodes. Nature, 374(6523), 627-629.

Delibas B., Arockiarajan A. y Seemann W. (2006): Rate dependent properties of perovskite type tetragonal piezoelectric materials using micromechanical model. International Journal of Solids and Structures, 43(3-4), 697-712.

Duan N., ten Elshof J.E., Verweij H., Greuel G. y Dannapple O. (2000): Enhancement of dielectric and ferroelectric properties by addition of Pt particles to a lead zirconate titanate matrix. Applied Physics Letters, 77(20), 3263.

Dunn M.L. y Taya M. (1993): Micromechanics predictions of the effective electroelastic moduli of piezoelectric composites. International Journal of Solids and Structures, $30(2), 161-175$.

Fiebig M. (2005): Revival of the magnetoelectric effect. Journal of Physics D: Applied Physics, 38(8), R123-R152.

Fleck N.A. y Willis J.R. (2004): Bounds and estimates for the effect of strain gradients upon the effective plastic properties of an isotropic two-phase composite. Journal of the Mechanics and Physics of Solids, 52(8), 1855-1888.

Francfort G.A., Giacomini A. y Musesti A. (2013): On the fleck and willis homogenization procedure in strain gradient plasticity. Discrete and Continuous Dynamical 
Systems - Series S, 6(1), 43-62.

Germain P., Nguyen Q.S. y Suquet P. (1983): Continuum Thermodynamics. Journal of Applied Mechanics, 50(4b), 1010.

Gurtin M.E., Fried E. y Anand L. (2010): The Mechanics and Thermodynamics of Continua. Cambridge University Press, Cambridge.

Hariton I. y DeBotton G. (2003): The nearly isotropic behaviour of high-rank nonlinear sequentially laminated composites. Proceedings of the Royal Society A: Mathematical, Physical and Engineering Sciences, 459(2029), 157-174.

Hashin Z. (2001): Thin interphase/imperfect interface in conduction. Journal of Applied Physics, 89(4), 2261-2267.

Hill R. (1956): New horizons in the mechanics of solids. Journal of the Mechanics and Physics of Solids, 5(1), 66-74.

Hill R. (1963): Elastic properties of reinforced solids: some theoretical principles. Journal of the Mechanics and Physics of Solids, 11(Hill 1962), 357-372.

Huber J.E. y Fleck N.A. (2001): Multi-axial electrical switching of a ferroelectric: theory versus experiment. Journal of the Mechanics and Physics of Solids, 49(4), 785-811.

Huber J.E. y Fleck N.A. (2004): Ferroelectric switching: A micromechanics model versus measured behaviour. European Journal of Mechanics, A/Solids, 23(2), 203-217.

Huber J.E., Fleck N.A., Landis C.M. y McMeeking R.M. (1999): A constitutive model for ferroelectric polycrystals. Journal of the Mechanics and Physics of Solids, 47(8), 1663-1697.

Hwang S.C. y McMeeking R.M. (1998): A finite element model of ferroelectric polycrystals. Ferroelectrics, 211(1), 177-194.

Idiart M.I. (2008): Modeling the macroscopic behavior of two-phase nonlinear composites by infinite-rank laminates. Journal of the Mechanics and Physics of Solids, 56(8), 2599-2617.

Idiart M.I. (2014): Modeling two-phase ferroelectric composites by sequential laminates. Modelling and Simulation in Materials Science and Engineering, 22(2), 25010, $1-20$.

Idiart M.I. y Castañeda P.P. (2007): Field statistics in nonlinear composites. I. Theory. Proceedings of the Royal Society A: Mathematical, Physical and Engineering Sciences, 463(2077), 183-202.

Idiart M.I. y Lahellec N. (2016): Estimates for the overall linear properties of pointwise heterogeneous solids with application to elasto-viscoplasticity. Journal of the Mechanics and Physics of Solids, 97, 317-332. 
Idiart M.I., Moulinec H., Ponte Castañeda P. y Suquet P. (2006): Macroscopic behavior and field fluctuations in viscoplastic composites: Second-order estimates versus full-field simulations. Journal of the Mechanics and Physics of Solids, 54(5), 1029-1063.

Idiart M.I. y Ponte Castañeda P. (2013): Estimates for two-phase nonlinear conductors via iterated homogenization. Proceedings of the Royal Society A: Mathematical, Physical and Engineering Sciences, 469(2153), 1-17.

Idiart M.I., Willot F., Pellegrini Y.P. y Ponte Castañeda P. (2009): Infinite-contrast periodic composites with strongly nonlinear behavior: Effective-medium theory versus full-field simulations. International Journal of Solids and Structures, 46(1819), 3365-3382.

IEEE Standards (1988): IEEE Standard on Piezoelectricity. ANSI/IEEE Std 176$198 \%$.

Kamlah M. (2001): Ferroelectric and ferroelastic piezoceramics - modeling of electromechanical hysteresis phenomena. Continuum Mechanics and Thermodynamics, 13(4), 219-268.

Kamlah M. y Böhle U. (2001): Finite element analysis of piezoceramic components taking into account ferroelectric hysteresis behavior. International Journal of Solids and Structures, 38(4), 605-633.

Kamlah M., Liskowsky A.C., McMeeking R.M. y Balke H. (2005): Finite element simulation of a polycrystalline ferroelectric based on a multidomain single crystal switching model. International Journal of Solids and Structures, 42(9-10), 29492964.

Kara H., Ramesh R., Stevens R. y Bowen C.R. (2003): Porous PZT ceramics for receiving transducers. IEEE Transactions on Ultrasonics, Ferroelectrics and Frequency Control, 50(3), 289-296.

Kawada S., Katayama R. y Horikawa K. (2010): Piezoelectric ceramic composition, method for manufacturing the same, and piezoelectric ceramic electronic component. US Patent 7691286 B2.

Keip M.A., Schrade D., Thai H., Schröder J., Svendsen B., Müller R. y Gross D. (2015): Coordinate-invariant phase field modeling of ferro-electrics, part II: Application to composites and poly-crystals. GAMM-Mitteilungen, 38(1), 115-131.

Kellogg R.a., Flatau a.B., Clark a.E., Wun-Fogle M. y Lograsso T.A. (2002): Temperature and stress dependencies of the magnetic and magnetostrictive properties of Fe0.81Ga0.19. Journal of Applied Physics, 91(10), 7821.

Kessler H. y Balke H. (2001): On the local and average energy release in polarization switching phenomena. Journal of the Mechanics and Physics of Solids, 49(5), 953-978. 
Klinkel S. (2006): A thermodynamic consistent 1D model for ferroelastic and ferroelectric hysteresis effects in piezoceramics. Communications in Numerical Methods in Engineering, 22(7), 727-739.

Kumazawa T., Kumagai Y., Miura H., Kitano M. y Kushida K. (1998): Effect of external stress on polarization in ferroelectric thin films. Applied Physics Letters, $72(5), 608-610$.

Labusch M., Keip M.A., Kiefer B. y Oder J.S. (2014): Computation of the effective magnetostrictive coefficient of magneto-mechanically coupled composites. En 11th World Congress on Computational Mechanics, WCCM XI, 1-12.

Lahellec N. y Suquet P. (2007): Effective behavior of linear viscoelastic composites: A time-integration approach. International Journal of Solids and Structures, 44(2), $507-529$.

Lahellec N. y Suquet P. (2013): Effective response and field statistics in elastoplastic and elasto-viscoplastic composites under radial and non-radial loadings. International Journal of Plasticity, 42, 1-30.

Lam K.S., Zhou Y., Wong Y.W. y Shin F.G. (2005): Electrostriction of lead zirconate titanate/polyurethane composites. Journal of Applied Physics, 97(104112).

Landis C.M. (2002): Fully coupled, multi-axial, symmetric constitutive laws for polycrystalline ferroelectric ceramics. Journal of the Mechanics and Physics of Solids, 50(1), 127-152.

Laskewitz B. y Kamlah M. (2010): Finite element implementation of nonlinear constitutive models for piezoceramic materials. Journal of Mechanics of Materials and Structures, 5(1), 19-45.

Lefèvre V. y Lopez-Pamies O. (2017): Nonlinear electroelastic deformations of dielectric elastomer composites: II - Non-Gaussian elastic dielectrics. Journal of the Mechanics and Physics of Solids, 99, 438-470.

Li J.F., Takagi K., Terakubo N. y Watanabe R. (2001): Electrical and mechanical properties of piezoelectric ceramic/metal composites in the $\mathrm{Pb}(\mathrm{Zr}, \mathrm{Ti}) \mathrm{O}_{3} / \mathrm{Pt}$ system. Applied Physics Letters, 79(15), 2441.

Linder C. y Miehe C. (2012): Effect of electric displacement saturation on the hysteretic behavior of ferroelectric ceramics and the initiation and propagation of cracks in piezoelectric ceramics. Journal of the Mechanics and Physics of Solids, $60(5), 882-903$.

Lines M.E. y Glass A.M. (1977): Principles and Applications of Ferroelectrics and Related Materials. International series of monographs on physics. OUP Oxford.

Lopez-Pamies O., Goudarzi T., Meddeb A.B. y Ounaies Z. (2014): Extreme enhancement and reduction of the dielectric response of polymer nanoparticulate composites via interphasial charges. Applied Physics Letters, 104(24), 242904. 
Macdonald J.R. (1959): Static Space Charge and Capacitance for Two-Blocking Electrodes. The Journal of Chemical Physics, 30(3), 806-816.

Mackey M., Schuele D.E., Zhu L., Flandin L., Wolak M.A., Shirk J.S., Hiltner A. y Baer E. (2012): Reduction of dielectric hysteresis in multilayered films via nanoconfinement. Macromolecules, 45(4), 1954-1962.

Mao Y.P., Mao S.Y., Ye Z.G., Xie Z.X. y Zheng L.S. (2010): Size-dependences of the dielectric and ferroelectric properties of $\mathrm{BaTiO}_{3} /$ polyvinylidene fluoride nanocomposites. Journal of Applied Physics, 108(1), 014102.

Marselli S., Pavia V., Galassi C., Roncari E., Craciun F. y Guidarelli G. (1999): Porous piezoelectric ceramic hydrophone. The Journal of the Acoustical Society of America, 106(2), 733.

Maugin G.A. y Daher N. (1986): Phenomenological theory of elastic semiconductors. International Journal of Engineering Science, 24(5), 703-731.

Maxwell J.C. (1873): A Treatise on Electricity and Magnetism, Vol. 1. A Treatise on Electricity and Magnetism. Clarendon Press, Cambridge.

McMeeking R.M. y Landis C.M. (2002): A phenomenological multi-axial constitutive law for switching in polycrystalline ferroelectric ceramics. International Journal of Engineering Science, 40(14), 1553-1577.

Mehling V., Tsakmakis C. y Gross D. (2007): Phenomenological model for the macroscopical material behavior of ferroelectric ceramics. Journal of the Mechanics and Physics of Solids, 55(10), 2106-2141.

Michel J.C., Moulinec H. y Suquet P. (2001): A computational scheme for linear and non-linear composites with arbitrary phase contrast. 5th U.S. National Congress on Computational Mechanics, 4-6 Aug. 1999, 52, 139-160.

Miehe C. (2002): Strain-driven homogenization of inelastic microstructures and composites based on an incremental variational formulation. International Journal for Numerical Methods in Engineering, 55(11), 1285-1322.

Miehe C., Kiefer B. y Rosato D. (2011a): An incremental variational formulation of dissipative magnetostriction at the macroscopic continuum level. International Journal of Solids and Structures, 48(13), 1846-1866.

Miehe C. y Rosato D. (2011): A rate-dependent incremental variational formulation of ferroelectricity. International Journal of Engineering Science, 49(6), 466-496.

Miehe C., Rosato D. y Kiefer B. (2011b): Variational principles in dissipative electromagneto-mechanics: A framework for the macro-modeling of functional materials. International Journal for Numerical Methods in Engineering, 86(10), 1225-1276.

Milton G.W. (2002): The Theory of Composites. Cambridge University Press, Cambridge. 
Moulinec H. y Suquet P. (1998): A numerical method for computing the overall response of nonlinear composites with complex microstructure. Computer Methods in Applied Mechanics and Engineering, 157(1-2), 69-94.

Munz D., Fett T., Muller S. y Thun G. (1998): Deformation and strength behaviour of a soft PZT ceramic. En V.V. Varadan, ed., Mathematics and Control in Smart Structures, vol. 3323, 84-95.

Nan C.W., Bichurin M.I., Dong S., Viehland D. y Srinivasan G. (2008): Multiferroic magnetoelectric composites: Historical perspective, status, and future directions. Journal of Applied Physics, 103(3).

Nie H.C., Dong X.L., Feng N.B., Chen X.F., Wang G.S., Gu Y., He H.L. y Liu Y.S. (2010): Quantitative dependence of the properties of $\mathrm{Pb}_{0,99}\left(\mathrm{Zr}_{0,95} \mathrm{Ti}_{0,05}\right)_{0,98} \mathrm{Nb}_{0,02} \mathrm{O}_{3}$ ferroelectric ceramics on porosity. Materials Research Bulletin, 45(5), 564-567.

Ning X., Yong Ping P. y Zhuo W. (2012): Large Dielectric Constant and MaxwellWagner Effects in $\mathrm{BaTiO}_{3} / \mathrm{Cu}$ Composites. Journal of the American Ceramic Society, 95(3), n/a-n/a.

O'Brien R. (1996): The Dielectric Response of a Dilute Suspension of Semiconducting Particles. Journal of colloid and interface science, 177(1), 280-281.

Olmos D., Martínez-Tarifa J.M., González-Gaitano G. y González-Benito J. (2012): Uniformly dispersed submicrometre $\mathrm{BaTiO}_{3}$ particles in PS based composites. Morphology, structure and dielectric properties. Polymer Testing, 31(8), 11211130 .

Pellegrini Y.P. (2001): Self-consistent effective-medium approximation for strongly nonlinear media. Physical Review B - Condensed Matter and Materials Physics, 64(13), 1-11.

Petchsuk A., Supmak W. y Thanaboonsombut A. (2011): Effects of Size of SprayDried PZT Powder and Dipole Density of Polymer Matrix on the Electrical Properties of PZT/Odd-Odd Nylons 0-3 Composites. Journal of the American Ceramic Society, 94(7), 2126-2134.

PI Piezo Technology (2017): Piezo Technology. https://www.piceramic.com/en/ piezo-technology/. Acceso: Jun 2017.

Piazza D., Galassi C., Barzegar A. y Damjanovic D. (2010): Dielectric and piezoelectric properties of PZT ceramics with anisotropic porosity. Journal of Electroceramics, 24(3), 170-176.

Ponte Castañeda P. (2016): Stationary variational estimates for the effective response and field fluctuations in nonlinear composites. Journal of the Mechanics and Physics of Solids, 96, 660-682.

Ponte Castañeda P. y Suquet P. (1998): Nonlinear Composites. Advances in Applied Mechanics, 34, 171-302. 
Priester L. (2013): Grain Boundaries, vol. 172 de Springer Series in Materials Science. Springer Netherlands, Dordrecht.

Ramesh R., Chan W.K., Wilkens B., Gilchrist H., Sands T., Tarascon J.M., Keramidas V.G., Fork D.K., Lee J. y Safari A. (1992): Fatigue and retention in ferroelectric Y-Ba-Cu-O/Pb-Zr-Ti-O/Y-Ba-Cu-O heterostructures. Applied Physics Letters, 61(13), 1537.

Randle V. y Owen G. (2006): Mechanisms of grain boundary engineering. Acta Materialia, 54(7), 1777-1783.

Ren X. (2004): Large electric-field-induced strain in ferroelectric crystals by pointdefect-mediated reversible domain switching. Nature Materials, 3(2), 91-94.

Rödel J., Kounga A.B., Aulbach E., Granzow T. y Leist T. (2007): Stress assisted electrical poling of ferroelectrics. IEEE International Symposium on Applications of Ferroelectrics, 638-640.

Saff E. y Kuijlaars A. (1997): Distributing many points on a sphere. The Mathematical Intelligencer, 19(1).

Schröder J. y Gross D. (2004): Invariant formulation of the electromechanical enthalpy function of transversely isotropic piezoelectric materials. Archive of Applied Mechanics, 73(8), 533-552.

Schröder J. y Romanowski H. (2005): A thermodynamically consistent mesoscopic model for transversely isotropic ferroelectric ceramics in a coordinate-invariant setting. Archive of Applied Mechanics, 74(11-12), 863-877.

Schwaab H., Deluca M., Supancic P. y Kamlah M. (2013): Effect of the electric conductivity on the modeling of the poling process of ferroelectric components. Journal of the Mechanics and Physics of Solids, 61(2), 504-516.

Scott J.F. (2000): Ferroelectric Memories, vol. 3 de Springer Series in Advanced Microelectronics. Springer Berlin Heidelberg, Berlin, Heidelberg.

Seemann W., Arockiarajan A. y Delibas B. (2004): Micromechanical simulation of piezoelectric materials using probability functions. En D.C. Lagoudas, ed., Proc.SPIE, vol. 5387, 402.

Senthil V., Badapanda T., Chithambararaj A., Chandra Bose A., Mohapatra A.K. y Panigrahi S. (2012): Dielectric relaxation behavior and electrical conduction mechanism in polymer-ceramic composites based on Sr modified Barium Zirconium Titanate ceramic. Journal of Polymer Research, 19(7), 9898.

Smith R.R.C., Seelecke S., Ounaies Z. y Smith J. (2003): A free energy model for hysteresis in ferroelectric materials. Journal of Intelligent Materials Systems and Structures, 14(11), 719-739.

Smyshlyaev V.P. y Willis J.R. (1998): A 'non-local' variational approach to the elastic 
energy minimization of martensitic polycrystals. Proceedings of the Royal Society A: Mathematical, Physical and Engineering Sciences, 454(1974), 1573-1613.

Sørensen T.S. (1997): Dielectric dispersion of a membrane consisting of a large number of solid bilayers containing mobile ions. Journal of the Chemical Society, Faraday Transactions, 93(24), 4327-4342.

Spinelli S.A. y Lopez-Pamies O. (2014): A general closed-form solution for the overall response of piezoelectric composites with periodic and random particulate microstructures. International Journal of Solids and Structures, 51(17), 2979-2989.

Stark S., Neumeister P. y Balke H. (2016a): A hybrid phenomenological model for ferroelectroelastic ceramics. Part I: Single phased materials. Journal of the Mechanics and Physics of Solids.

Stark S., Neumeister P. y Balke H. (2016b): Some aspects of macroscopic phenomenological material models for ferroelectroelastic ceramics. International Journal of Solids and Structures, 80, 359-367.

Suryanarayana P. y Bhattacharya K. (2012): Evolution of polarization and space charges in semiconducting ferroelectrics. Journal of Applied Physics, 111(3), 034109 .

Takagi K., Li J.F., Yokoyama S. y Watanabe R. (2003): Fabrication and evaluation of PZT/Pt piezoelectric composites and functionally graded actuators. Journal of the European Ceramic Society, 23(10), 1577-1583.

Talbot D.R.S. y Willis J.R. (1985): Variational principles for inhomogeneous nonlinear media. IMA Journal of Applied Mathematics (Institute of Mathematics and Its Applications), 35(1), 39-54.

Talbot D.R.S. y Willis J.R. (1986): A variational approach to the overall sink strength of a nonlinear lossy composite medium. Proceedings of the Royal Society A: Mathematical, Physical and Engineering Sciences, 405(1828), 159-180.

Tan W.L. y Kochmann D.M. (2017): An effective constitutive model for polycrystalline ferroelectric ceramics: Theoretical framework and numerical examples. Computational Materials Science, 136, 223-237.

Tanaka R., Yamaguchi H. y Yura Y. (2012): Piezoelectric/electrostrictive ceramic composition manufacturing method. US Patent 8119023 B2.

Tartar L. (1990): H-measures, a new approach for studying homogenisation, oscillations and concentration effects in partial differential equations. Proceedings of the Royal Society of Edinburgh: Section A Mathematics, 115(3-4), 193-230.

Tonkoshkur A., Glot A. y Ivanchenko A. (2017): Basic models in dielectric spectroscopy of heterogeneous materials with semiconductor inclusions. Multidiscipline Modeling in Materials and Structures, 13(1), 36-57. 
Topolov V.Y. y Bowen C.R. (2009): Electromechanical Properties in Composite Based on Ferroelectrics. Engineering Materials and Processes. Springer London, London.

Trukhan E. (1963): Dispersion of the dielectric constant of heterogeneous systems. Soviet Physics - Solid State, 4(12), 2560-2570.

Uchino K. (2010): Ferroelectric devices. CRC Press, Inc., 2da edición.

van den Ende D.A., van de Wiel H.J., Groen W.A. y van der Zwaag S. (2012): Direct strain energy harvesting in automobile tires using piezoelectric PZT-polymer composites. Smart Materials and Structures, 21(1), 15011.

Wang J., Shu W., Fang H. y Kamlah M. (2014): Phase field simulations of the poling process and nonlinear behavior of ferroelectric polycrystals with semiconducting grain boundaries. Smart Materials and Structures, 23(9), 095016.

Wauer J. y Suherman S. (1997): Thickness vibrations of a piezo-semiconducting plate layer. 35(15), 1387-1404.

Xiao Y. y Bhattacharya K. (2001): Modeling electromechanical properties of ionic polymers. En Y. Bar-Cohen, ed., Proc. SPIE, vol. 4329, 292.

Xiao Y. y Bhattacharya K. (2008): A Continuum Theory of Deformable, Semiconducting Ferroelectrics. Archive for Rational Mechanics and Analysis, 189(1), 59-95.

Xu Y. (1991): Ferroelectric Materials and their applications. North-Holland Elsevier Science, Amsterdam.

Yang J.S. y Zhou H.G. (2005): Amplification of acoustic waves in piezoelectric semiconductor plates. International Journal of Solids and Structures, 42(11-12), 3171-3183.

Zang G., Zhang J., Zheng P., Wang J. y Wang C. (2005): Grain boundary effect on the dielectric properties of $\mathrm{CaCu}_{3} \mathrm{Ti}_{4} \mathrm{O}_{12}$ ceramics. Journal of Physics D: Applied Physics, 38(11), 1824-1827.

Zeng T., Dong X., Mao C., Zhou Z. y Yang H. (2007): Effects of pore shape and porosity on the properties of porous PZT 95/5 ceramics. Journal of the European Ceramic Society, 27(4), 2025-2029.

Zhai J., Xing Z., Dong S., Li J. y Viehland D. (2008): Magnetoelectric laminate composites: An overview. Journal of the American Ceramic Society, 91(2), 351-358.

Zhang H., Yang S., Zhang B.P. y Li J.F. (2010a): Electrical properties of Ni-particledispersed alkaline niobate composites sintered in a protective atmosphere. Materials Chemistry and Physics, 122(1), 237-240.

Zhang Q.M., Corkovic S., Shaw C.P., Huang Z. y Whatmore R.W. (2005): Effect of porosity on the ferroelectric properties of sol-gel prepared lead zirconate titanate 
thin films. Thin Solid Films, 488(1-2), 258-264.

Zhang S.T., Chen Z., Zhang C. y Yuan G.L. (2010b): Temperature-dependent ferroelectric and dielectric properties of $\mathrm{Bi}_{3,25} \mathrm{La}_{0,75} \mathrm{Ti}_{3} \mathrm{O}_{12}$ thin films. Applied Surface Science, 256(8), 2468-2473.

Zhang W. y Bhattacharya K. (2005): A computational model of ferroelectric domains. Part I: model formulation and domain switching. Acta Materialia, 53(1), 185-198.

Zhang Y., Zhong X.L., Chen Z.H., Wang J.B. y Zhou Y.C. (2011): Temperature dependence of polarization switching properties of $\mathrm{Bi}_{3,15} \mathrm{Nd}_{0,85} \mathrm{Ti}_{3} \mathrm{O}_{12}$ ferroelectric thin film. Journal of Applied Physics, 110(1), 014102.

Zhou D., Kamlah M. y Munz D. (2001): Rate dependence of soft PZT ceramics under electric field loading. En C.S. Lynch, ed., Smart Structures and Materials 2001: Active Materials: Behavior and Mechanics, vol. 4333, 64-70.

Zhou D., Kamlah M. y Munz D. (2005): Effects of uniaxial prestress on the ferroelectric hysteretic response of soft PZT. Journal of the European Ceramic Society, 25(4), 425-432.

Zhou W., Dong L., Sui X., Wang Z., Zuo J., Cai H. y Chen Q. (2016): High dielectric permittivity and low loss in PVDF filled by core-shell Zn@ZnO particles. Journal of Polymer Research, 23(3), 45. 


\section{Anexos}




\section{A Cálculos para materiales laminados con cargas libres}

Se considera una celda unitaria compuesta de dos láminas consecutivas de fase $r=1$ y $r=2$. Sea $y$ la coordenada normal de modo tal que la capa de la fase $r=1$ ocupa el dominio $\left[0, L^{(1)}\right]$ mientras que la capa de fase $r=2$ ocupa el dominio $\left[L^{(1)}, L\right]$ con $L=L^{(1)}+L^{(2)}$, ver figura 7.1a.

\section{A.1 Respuesta lineal}

Las ecuaciones de Euler-Lagrange de (6.72) son

$$
-\left[\epsilon(y) \phi^{\prime}(y)\right]^{\prime}+\sum_{r=1}^{2} \chi^{(r)}(y) \frac{F^{2} z^{2} c_{-}^{(r)}}{R T}\left(\langle\phi\rangle^{(r)}-\phi(y)\right)=0,
$$

donde $\chi^{(r)}$ es la función característica del dominio ocupado por la fase $r$, y la función $\phi(y)+\bar{E} y$ es periódica en $L$. Entonces, dentro de cada capa el potencial electrostático cumple con la ecuación diferencial de equilibrio

$$
-\ell^{(r)^{2}} \phi^{\prime \prime}(y)+\phi(y)=\langle\phi\rangle^{(r)},
$$

cuya solución general es de la forma

$$
\phi(y)=C_{1}^{(r)} \cosh \left(\frac{y}{\ell^{(r)}}\right)+C_{2}^{(r)} \sinh \left(\frac{y}{\ell^{(r)}}\right)+\langle\phi\rangle^{(r)} .
$$

Las seis constantes $C_{i}^{(r)}$ y $\langle\phi\rangle^{(r)}$ surgen de las dos condiciones de promedio

$$
C_{1}^{(r)}\left\langle\cosh \left(\frac{y}{\ell^{(r)}}\right)\right\rangle^{(r)}+C_{2}^{(r)}\left\langle\sinh \left(\frac{y}{\ell^{(r)}}\right)\right\rangle^{(r)}=0
$$

para $r=1,2$, las condiciones de continuidad del potencial electrostático y del desplazamiento eléctrico normal en la interfase

$$
\begin{array}{r}
C_{1}^{(1)} \cosh \left(\frac{L^{(1)}}{\ell^{(1)}}\right)+C_{2}^{(1)} \sinh \left(\frac{L^{(1)}}{\ell^{(1)}}\right)+\langle\phi\rangle^{(1)}= \\
C_{1}^{(2)} \cosh \left(\frac{L^{(1)}}{\ell^{(2)}}\right)+C_{2}^{(2)} \sinh \left(\frac{L^{(1)}}{\ell^{(2)}}\right)+\langle\phi\rangle^{(2)}
\end{array}
$$


y

$$
\begin{gathered}
\frac{\epsilon^{(1)}}{\ell^{(1)}}\left[C_{1}^{(1)} \sinh \left(\frac{L^{(1)}}{\ell^{(1)}}\right)+C_{2}^{(1)} \cosh \left(\frac{L^{(1)}}{\ell^{(1)}}\right)\right]= \\
\frac{\epsilon^{(2)}}{\ell^{(2)}}\left[C_{1}^{(2)} \sinh \left(\frac{L^{(1)}}{\ell^{(2)}}\right)+C_{2}^{(2)} \cosh \left(\frac{L^{(1)}}{\ell^{(2)}}\right)\right],
\end{gathered}
$$

la condición de periodicidad

$$
C_{1}^{(1)}+\langle\phi\rangle^{(1)}=C_{1}^{(2)} \cosh \left(\frac{L}{\ell^{(2)}}\right)+C_{2}^{(2)} \sinh \left(\frac{L}{\ell^{(2)}}\right)+\langle\phi\rangle^{(2)}+\bar{E} L,
$$

y una condición definiendo la constante arbitraria, tal como

$$
L^{(1)}\langle\phi\rangle^{(1)}+L^{(2)}\langle\phi\rangle^{(2)}=0 .
$$

Una vez que el sistema de seis ecuaciones lineales (A.4)-(A.8) es resuelto para encontrar las constantes, el campo potencial electrostático queda especificado completamente por la expresión (A.3).

\section{A.2 Respuesta no lineal}

Cada fase $r$ se discretiza en $N_{e}^{(r)}$ elementos de modo que el funcional en (6.69) se convierte en

$$
\begin{aligned}
U= & \sum_{r=1}^{2} f^{(r)}\left\{\frac{1}{2} \epsilon^{(r)} \frac{1}{N_{e}^{(r)}} \sum_{i \in I^{(r)}} E_{i}^{2}+\right. \\
& \left.+R T c_{-}^{(r)} \ln \left[\frac{1}{N_{e}^{(r)}} \sum_{i \in I^{(r)}} \exp \left(-\frac{F z}{R T}\left(\phi_{i}-\bar{\phi}^{(r)}\right)\right)\right]\right\}
\end{aligned}
$$

donde

$$
\phi_{i}=\frac{\phi_{i}^{[2]}+\phi_{i}^{[1]}}{2}, \quad E_{i}=N_{e}^{(r)} \frac{\phi_{i}^{[2]}-\phi_{i}^{[1]}}{L^{(r)}}, \quad \bar{\phi}^{(r)}=\frac{1}{N_{e}^{(r)}} \sum_{i \in I^{(r)}} \phi_{i} .
$$

En estas expresiones, $\phi_{i}^{[1]}$ y $\phi_{i}^{[2]}$ se refieren al campo potencial electrostático en los puntos nodales del elemento $i$ con coordenadas espaciales $y_{i}^{[1]}$ y $y_{i}^{[2]}=y_{i}^{[1]}+\left(L^{(r)} / N_{e}^{(r)}\right)$, respectivamente, y el conjunto de índices $I^{(r)}$ contiene a los índices de los elementos dentro de la fase $r$. La constante arbitraria y las condiciones de periodicidad se fijan estableciendo $\phi(0)=0$ y $\phi(L)=-\bar{E} L$.

Para un campo macroscópico $\bar{E}$ dado, el funcional discretizado (A.9) se minimiza con respecto a los valores nodales del potencial electrostático por medio del algoritmo de cuasi Newton BFGS. Los resultados presentados en la sección 7.2.2 consideran un elemento para la fase dieléctrica y sesenta elementos para la fase semiconductora. 


\section{B Cálculos para materiales particulados con cargas libres}

\section{B.1 Matriz con partículas en suspensión diluida}

Se considera una inclusión esférica de radio $a$ de fase $r=2$ en un medio infinito hecho de fase $r=1$, ver figura 7.4a. El potencial electrostático es solución de

$$
-\nabla \cdot(\epsilon(\mathbf{y}) \nabla \phi)+\chi^{(2)}(\mathbf{y}) \frac{F^{2} z^{2} c_{-}^{(2)}}{R T}\left(\langle\phi\rangle^{(2)}-\phi\right)=0
$$

donde $\chi^{(2)}(\mathbf{y})$ es la función característica del dominio ocupado por la partícula, y $\phi(\mathbf{y})+\overline{\mathbf{E}} \cdot \mathbf{y} \rightarrow 0$ cuando $|\mathbf{y}| \rightarrow \infty$.

Para una intensidad de campo eléctrico macroscópico $\overline{\mathbf{E}}$ dada, se selecciona un sistema de coordenadas esférico con origen en el centro de la partícula y eje normal a lo largo de la dirección de $\overline{\mathbf{E}}$; se denomina $\rho$ a la coordenada radial y $\varphi$ al ángulo azimutal. Dada la simetría del problema, el potencial electrostático no varía con el ángulo polar. Entonces, en vistas de las ecuaciones (B.1), el potencial electrostático es solución de

$$
\frac{\partial}{\partial \rho}\left(\rho^{2} \frac{\partial \phi}{\partial \rho}\right)+\frac{1}{\sin \varphi} \frac{\partial}{\partial \varphi}\left(\sin \varphi \frac{\partial \phi}{\partial \varphi}\right)=0
$$

dentro del dominio $\Omega^{(1)}$ de la matriz, y de

$$
-\frac{\ell^{2}}{\rho^{2}}\left[\frac{\partial}{\partial \rho}\left(\rho^{2} \frac{\partial \phi}{\partial \rho}\right)+\frac{1}{\sin \varphi} \frac{\partial}{\partial \varphi}\left(\sin \varphi \frac{\partial \phi}{\partial \varphi}\right)\right]+\phi=\langle\phi\rangle^{(2)}
$$

dentro del dominio $\Omega^{(2)}$ de la partícula, donde $\ell$ denota la longitud de Debye de la fase de la inclusión. Estas ecuaciones admiten soluciones de la forma

$$
\phi(\rho, \varphi)=C_{0}^{(r)}+f^{(r)}(\rho) \cos \varphi
$$

donde

$$
f^{(1)}(\rho)=C_{1}^{(1)} \rho+\frac{C_{2}^{(1)}}{\rho^{2}}
$$


dentro de $\Omega^{(1)}, \mathrm{y}$

$$
\begin{aligned}
f^{(2)}(\rho)= & C_{1}^{(2)}\left[(\ell / \rho)^{2} \cosh (\rho / \ell)-(\ell / \rho) \sinh (\rho / \ell)\right]+ \\
& +C_{2}^{(2)}\left[(\ell / \rho) \cosh (\rho / \ell)-(\ell / \rho)^{2} \sinh (\rho / \ell)\right]
\end{aligned}
$$

dentro de $\Omega^{(2)}$. Ahora bien, el carácter acotado de $\phi$ en el origen y la condición de borde en el infinito requieren que

$$
C_{1}^{(1)}=-|\overline{\mathbf{E}}| \quad \mathrm{y} \quad C_{1}^{(2)}=0
$$

mientras que las condiciones de continuidad del potencial electrostático y del desplazamiento eléctrico normal a la interfase partícula-matriz requieren que

$$
\begin{aligned}
C_{0}^{(1)} & =C_{0}^{(2)}, \\
-|\overline{\mathbf{E}}| a^{3}+C_{2}^{(1)} & =C_{2}^{(2)} \ell^{2}[(a / \ell) \cosh (a / \ell)-\sinh (a / \ell)] \quad \mathrm{y} \\
\epsilon^{(1)}\left[|\overline{\mathbf{E}}| a^{3}+2 C_{2}^{(1)}\right] & =C_{2}^{(2)} \epsilon^{(2)} \ell^{2}\left[2 \frac{a}{\ell} \cosh (a / \ell)-\left(2+(a / \ell)^{2}\right) \sinh (a / \ell)\right] .
\end{aligned}
$$

Finalmente, una condición conveniente para fijar la constante arbitraria es

$$
C_{0}^{(1)}=0
$$

Por lo tanto, las expresiones (B.9) y (B.10) constituyen un sistema de dos ecuaciones lineales para las constantes $C_{1}^{(2)}$ y $C_{2}^{(2)}$. Una vez que estas constantes son determinadas, el campo potencial electrostático se encuentra completamente definido por las expresiones (B.4)-(B.6).

\section{B.2 Matriz con partículas en concentraciones arbitrarias}

Se considera una inclusión esférica de radio $a$ de fase $r=2$, recubierta por una capa de fase $r=1$ en un medio infinito hecho de fase $r=0$. Se considera que en el medio que contiene las partículas tiene el valor de la permitividad equivalente $\bar{\epsilon}$ y el campo eléctrico es uniforme, con valor $\overline{\mathbf{E}}$ (ver figura $7.4 \mathrm{~b}$ ). El potencial electrostático es solución de

$$
-\nabla \cdot(\epsilon(\mathbf{y}) \nabla \phi)+\sum_{r=0}^{2} \chi^{(r)}(\mathbf{y}) \frac{F^{2} z^{2} c_{-}^{(r)}}{R T}\left(\langle\phi\rangle^{(r)}-\phi(\mathbf{y})\right)=0,
$$

donde $\chi^{(r)}$ es la función característica del dominio ocupado por la fase $r, \mathrm{y} \phi(\mathbf{y})+$ $\overline{\mathbf{E}} \cdot \mathbf{y} \rightarrow 0$ cuando $|\mathbf{y}| \rightarrow \infty$

Para una intensidad de campo eléctrico macroscópico $\overline{\mathbf{E}}$ dada, se selecciona un 
sistema de coordenadas esférico con origen en el centro de la partícula y eje normal a lo largo de la dirección de $\overline{\mathbf{E}}$; se denomina $\rho$ a la coordenada radial y $\varphi$ al ángulo azimutal. Dada la simetría del problema, el potencial electrostático no varía con el ángulo polar. Entonces, en vistas de las ecuaciones (B.12), el potencial electrostático es solución de

$$
\frac{\partial}{\partial \rho}\left(\rho^{2} \frac{\partial \phi}{\partial \rho}\right)+\frac{1}{\sin \varphi} \frac{\partial}{\partial \varphi}\left(\sin \varphi \frac{\partial \phi}{\partial \varphi}\right)=0
$$

dentro del dominio $\Omega^{(0)} \cup \Omega^{(1)}$ de la matriz y el recubrimiento, y de

$$
-\frac{\ell^{2}}{\rho^{2}}\left[\frac{\partial}{\partial \rho}\left(\rho^{2} \frac{\partial \phi}{\partial \rho}\right)+\frac{1}{\sin \varphi} \frac{\partial}{\partial \varphi}\left(\sin \varphi \frac{\partial \phi}{\partial \varphi}\right)\right]+\phi=\langle\phi\rangle^{(2)}
$$

dentro del dominio $\Omega^{(2)}$ de la partícula, donde $\ell$ denota la longitud de Debye de la fase de la inclusión. Estas ecuaciones admiten soluciones de la forma

$$
\phi(\rho, \varphi)=C_{0}^{(r)}+f^{(r)}(\rho) \cos \varphi
$$

donde

$$
f^{(0)}(\rho)=C_{1}^{(0)} \rho+\frac{C_{2}^{(0)}}{\rho^{2}}
$$

dentro de $\Omega^{(0)}$,

$$
f^{(1)}(\rho)=C_{1}^{(1)} \rho+\frac{C_{2}^{(1)}}{\rho^{2}}
$$

dentro de $\Omega^{(1)}$, y

$$
\begin{aligned}
f^{(2)}(\rho)= & C_{1}^{(2)}\left[(\ell / \rho)^{2} \cosh (\rho / \ell)-(\ell / \rho) \sinh (\rho / \ell)\right]+ \\
& +C_{2}^{(2)}\left[(\ell / \rho) \cosh (\rho / \ell)-(\ell / \rho)^{2} \sinh (\rho / \ell)\right]
\end{aligned}
$$

dentro de $\Omega^{(2)}$. Ahora bien, el carácter acotado de $\phi$ en el origen y la condición de borde en el infinito requieren que

$$
C_{1}^{(0)}=-|\overline{\mathbf{E}}| \quad \mathrm{y} \quad C_{1}^{(2)}=0,
$$

mientras que las condiciones de continuidad del potencial electrostático y del desplazamiento eléctrico normal a las interfases partícula-recubrimiento y recubrimiento- 
matriz requieren que

$$
\begin{aligned}
C_{0}^{(0)} & =C_{0}^{(1)}, \\
C_{0}^{(1)} & =C_{0}^{(2)}, \\
-|\overline{\mathbf{E}}| b^{3} & =C_{1}^{(1)} b^{3}+C_{2}^{(1)} \\
\bar{\epsilon}|\overline{\mathbf{E}}| b^{3} & =-\epsilon^{(1)}\left(C_{1}^{(1)} b^{3}-2 C_{2}^{(1)}\right), \\
C_{1}^{(1)} a^{3}+C_{2}^{(1)} & =C_{2}^{(2)} \ell^{2}[(a / \ell) \cosh (a / \ell)-\sinh (a / \ell)] \quad \mathrm{y} \\
\epsilon^{(1)}\left(C_{1}^{(1)} a^{3}-2 C_{2}^{(1)}\right) & =C_{2}^{(2)} \epsilon^{(2)} \ell^{2}[2(a / \ell) \cosh (a / \ell)- \\
& \left.-\left(2+(a / \ell)^{2}\right) \sinh (a / \ell)\right] .
\end{aligned}
$$

Finalmente, una condición conveniente para fijar la constante arbitraria es

$$
C_{0}^{(2)}=0
$$

Por lo tanto, las expresiones (B.22)-(B.25) constituyen un sistema de cuatro ecuaciones lineales para las constantes $C_{1}^{(1)}, C_{2}^{(1)}, C_{2}^{(2)}$ y la permitividad efectiva $\bar{\epsilon}$. Una vez que estas constantes son determinadas, el campo potencial electrostático se encuentra completamente definido por las expresiones (B.15)-(B.18). 
